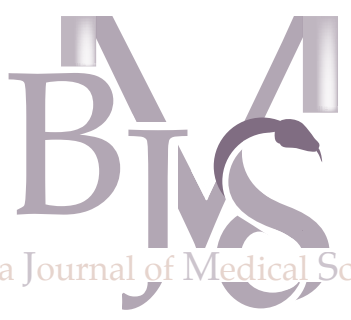

Batna Journal of

Medical Sciences
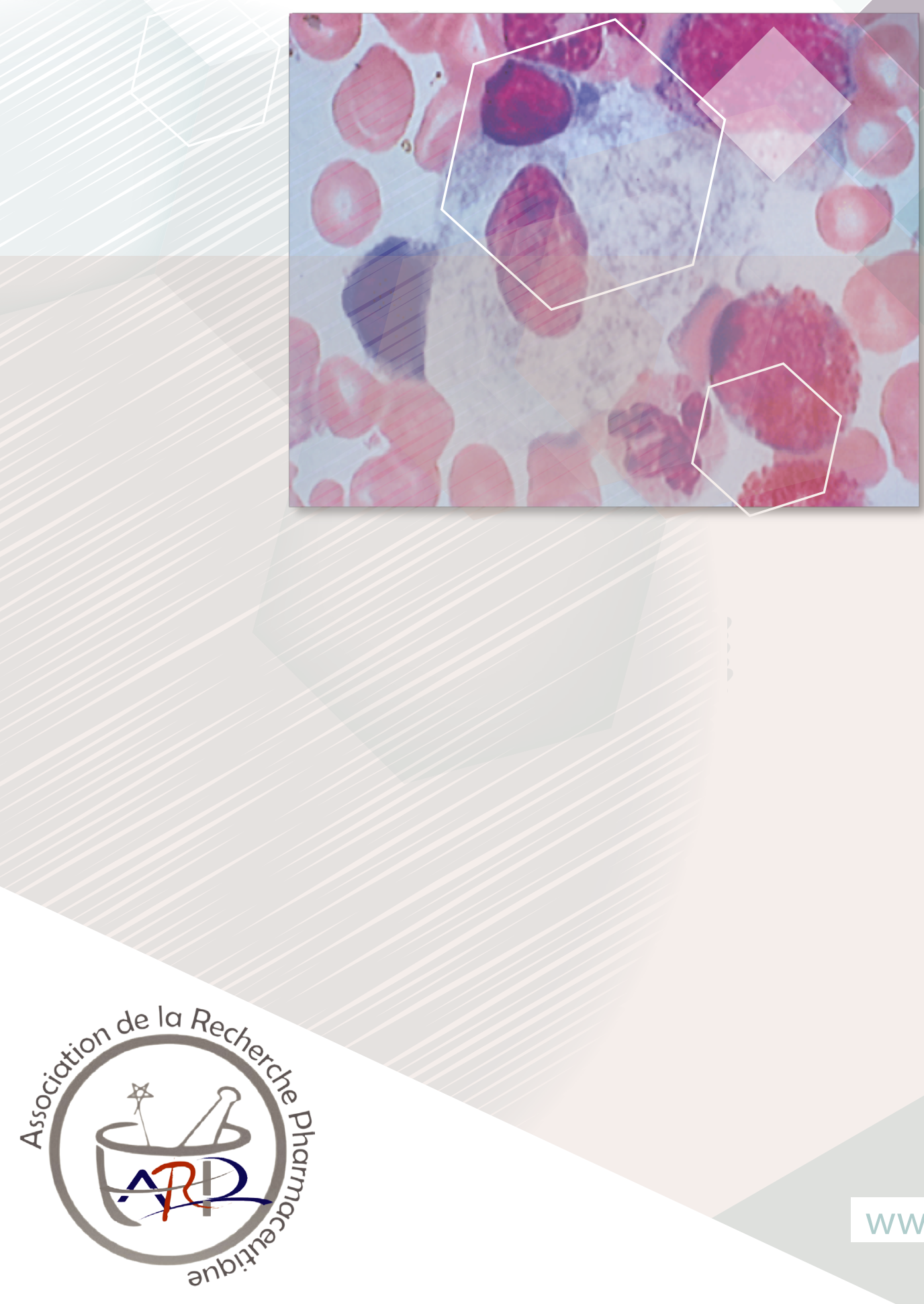


\section{Batna Journal Of Medical Sciences}

\section{Organe officiel de l'association de la Recherche \\ Pharmaceutique}

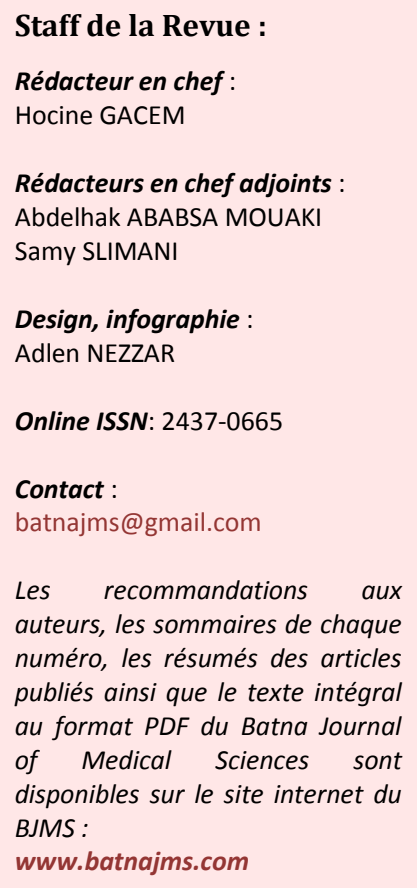

Volume 2, Numéro 1 - Juin 2015. Pages 1-110

TABLE DES MATIÈRES

Editorial

Batna Journal of Medical Sciences évolue

S Slimani

page 1

Mises au point

Epilepsie, violence et responsabilité pénale

Z Benmebarek

pages 2-6

Actualités sur la vitamine $\mathrm{D}$

M Djennane, JC Soubrielle

pages $7-12$

Diagnostic de la broncho-pneumopathie chronique obstructive asymptomatique par spirométrie

$S$ Ferhi, B Kermiche, $N$ Chibout, MR Guedjati

pages $13-18$

Apports sodés et hypertension artérielle

M Mansouri

pages 19-23

Peau et soleil

$R$ Zerguine

pages $24-29$

Articles originaux

L'automédication par les antibiotiques : étude auprès de cinq officines pharmaceutiques de cinq villes algériennes A Aggabi, H Gacem, H Bahri, B Talha, A Boulerial, A Gacem, Y Koubaa, A Yahiaoui, A Ahmane $\quad$ pages 30-35

Brèves de congrès

Brèves du congrès de l'IOF-ESCEO 2015. Congrès mondial de l'ostéoporose, de l'arthrose et des affections musculosquelettiques

S Slimani

pages $36-38$

Dossier thématique : génétique

Editorial : La génétique humaine : plus qu'un simple engouement, une nécessité !

G Belaaloui

page 39

Les mucopolysaccharidoses

A Benmansour

pages $40-44$

La maladie de Crohn : entre le déterminisme génétique et l'espoir épigénétique

G Belaaloui

pages $45-49$

La génétique et l'épigénétique au service des maladies rénales

$M$ Cherif Benmoussa, $R$ Grari, $R$ Sari Hamidou, M Benmansour

pages $50-55$

Epigénétique et pathogénèse du diabète de type 2

A Gouri, M Bouchareb, A Dekaken, AA Bentorki, M Chefrour, R Guieu, S Benharkat

Biologie moléculaire et cancer de la thyroïde

A Nezzar

pages $56-59$

Maladie de Gaucher

F Rahal, N Brahimi, A Ladjouze-Rezig

La maladie génétique et la famille : fatalité ou culpabilité ?

H Salhi

pages 60-65

pages 66-69

pages $70-74$

Cas cliniques

Traitement d'une neurosarcoïdose infundibulaire par corticoïdes

$S$ Azzoug, F Chentli

pages $75-77$

Infanticide au décours d'une psychose du post-partum

Z Benmebarek

pages $78-81$

Kyste hydatique du psoas: à propos d'un cas

O Hamouda, M Benchikh, $N$ Boudjouraf

pages $82-84$

Endométriose colique : à propos de 2 cas

W Merzouki, S Khouni, A Benazza, O Bafdel, A Hadj Brahim, S Guerfi, H Khadraoui

Infarctus musculaire et diabète

W Rachidi, K Nassar, S Janani, O Mkinsi

pages $85-88$

pages $89-92$

Arrêt sur image

Spondyloarthrite diagnostiquée lors du bilan d'une maladie de Crohn compliquée

A Salah Salah

page 93

Abstracts de Congrès

$1^{\text {ère }}$ Conférence Internationale de Guelma sur les hépatites (CIGH)

Université de Guelma, 9 avril 2015 


\section{Batna Journal of Medical Sciences évolue.}

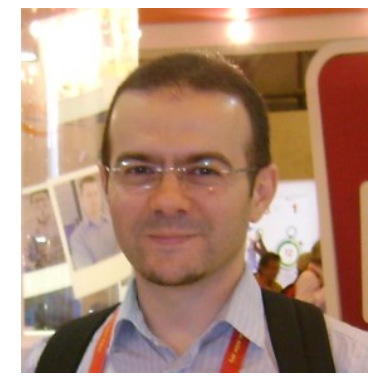

Correspondance à :

Samy SLIMANI

slimani@dr.com

DOI :https://doi.org/10.48087/ BIMSe.2015.2101

Il s'agit d'un article en libre accès distribué selon les termes de la licence Creative Commons Attribution International License (CC BY 4.0), qui autorise une utilisation, une distribution et une reproduction sans restriction sur tout support ou format, à condition que l'auteur original et la revue soient dûment crédités.

Pour citer l'article :

Slimani S. Batna Journal of Medical Sciences évolue. Batna J Med Sci 2015;2(1):1.

https://doi.org/10.48087/ BJMSe.2015.2101

\section{Chers lecteurs, chères lectrices,}

Je suis très heureux de vous présenter le numéro régulier de juin de Batna Journal of Medical Sciences (BJMS) qui est le premier de l'année 2015, cette année qui s'annonce très bonne.

Mais tout d'abord, je suis honoré de passer le flambeau à mon ami le Dr. Hocine Gacem, qui a pris la rédaction-en-chef de la revue pour cette année 2015, poste que j'occupais durant la première année d'existence de BJMS, durant laquelle la revue a pu démarrer et se faire connaître à travers ses deux numéros semestriels et son supplément de septembre, totalisant 41 articles publiés, provenant des quatre coins du pays.

L'année 2015 s'annonce prometteuse à plusieurs titres :

L'indexation: après une indexation le 10 mars 2015 dans la base de données Google Scholar, une deuxième indexation est réalisée par l'attribution d'un International Standard Serial Number (ISSN). Nous projetons de poursuivre les indexations dans les bases de données Open Access avant de rejoindre les bases majeures que sont SCOPUS et PubMed.

Le nombre de soumissions: alors que nous n'avons reçu que 10 soumissions d'articles durant le premier semestre 2014, ce chiffre est monté à 30 soumissions durant le dernier semestre, provenant de 3 pays (Algérie, Maroc, France). Nous sommes fiers qu'en plus de cette dimension internationale de la revue qui est en train de se dessiner, la moitié des articles provient toujours d'auteurs locaux (exerçant dans la wilaya de Batna). Nous ne remercierons jamais assez les différents auteurs pour leur confiance et d'avoir cru en la réussite de BJMS.

La visibilité: La revue est de plus en plus visible, en atteste le nombre grandissant des soumissions, mais aussi le nombre de personnes suivant notre page Facebook (1500 fans) ainsi que le nombre de personnes ayant consulté les différents articles de la revue sur son site web www.batnajms.com, qui a totalisé 18000 visites ce dernier semestre, en augmentation de $300 \%$ par rapport au précédent semestre.

Ce numéro régulier de juin 2015 intègre deux nouvelles rubriques : brèves de congrès et abstracts de congrès. Nous invitons toute personne intéressée par la mise en publication des abstracts de journées biomédicales à se rapprocher de la rédaction, et nous nous ferons un plaisir de formater les abstracts et les inclure en supplément ou dans un numéro régulier de la revue.

Comme à l'accoutumée, est incluse une monographie de 10 articles portant sur le sujet très intéressant et de plus en plus d'actualité qui est la génétique, avec une équipe d'auteurs très motivée, coordonnée par notre excellente collègue le Dr. Ghania Belaaloui.

Je vous laisse découvrir ce numéro en vous souhaitant une bonne lecture, et en restant à votre disposition pour toute suggestion ou commentaire.

Dr Samy SLIMANI Rédacteur en Chef Adjoint

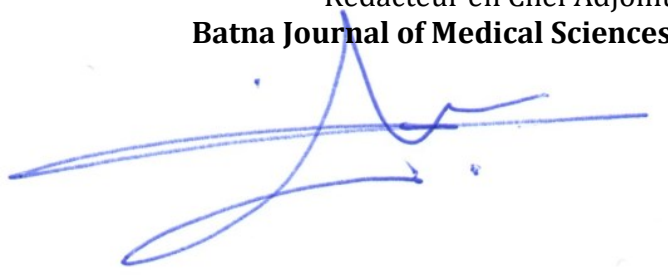




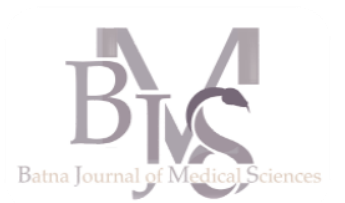

Service de psychiatrie, EHS psychiatrique El Madher, Batna - Algérie

\section{Correspondance à :}

Zoubir BENMEBAREK

zoubirbenmeb@gmail.com

DOI :https://doi.org/10.48087/ BJMSra.2015.2102

\section{Il s'agit d'un article en libre} accès distribué selon les termes de la licence Creative Commons Attribution International License (CC BY 4.0), qui autorise une utilisation, une distribution et une reproduction sans restriction sur tout support ou format, à condition que l'auteur original et la revue soient dûment crédités.

\section{Épilepsie, violence et responsabilité pénale}

\section{Epilepsy, violence and penal responsibility}

\section{Zoubir Benmebarek}

\section{RÉSUMÉ}

L'épilepsie a été associée pendant longtemps à la violence et à la criminalité mais cette notion est actuellement remise en cause, car il est admis que d'autres facteurs contextuels entrent en jeu pour expliquer ce phénomène. L'analyse de la violence chez un épileptique passe par la connaissance clinique approfondie des différents syndromes épileptiques et des circonstances de survenue du comportement agressif. L'épileptique reste pénalement responsable sauf dans des situations exceptionnelles où les crises épileptiques peuvent engendrer un comportement violent ou agressif généralement mineur et non intentionnel.

Mots-clés : Épilepsie, violence, criminalité, responsabilité pénale.

\begin{abstract}
Violence has been for a long time considered to be linked to epilepsy but modern studies refute this view. Violence in epileptic patients is a complex phenomenon with multifaceted causes. It is well established now that there is no link between epilepsy and criminal behavior and that epileptics are liable for their deeds. The forensic evaluation for criminal responsibility of an epileptic who committed an offense is carried through a thorough knowledge of epileptic syndromes and a detailed analysis of behavior during the offense.
\end{abstract}

Keywords: epilepsy, violence, criminality, criminal responsibility.

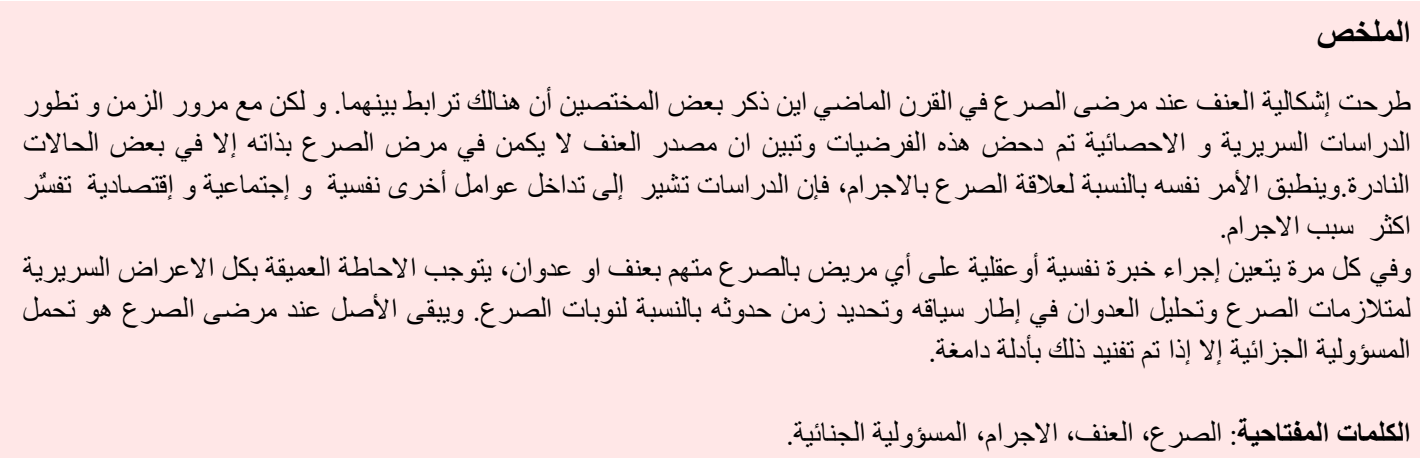

\section{INTRODUCTION}

La violence due à l'épilepsie est une notion controversée; elle est répandue aussi bien parmi les gens ordinaires que parmi les médecins bien que les études cliniques sur ce sujet n'ont jamais affirmé ce constat, sauf quelques cas isolés rapportés par la littérature médicale [1]. La rage ou fureur épileptique où l'individu se déchaîne dans une violence incontrôlable et inconsciente pouvant atteindre le meurtre est encore présente dans certains manuels de psychiatrie $[2,3]$.

L'épilepsie, qui est une atteinte cérébrale, est caractérisée par une prédisposition persistante à la production de crises épileptiques, et par les conséquences neurobiologiques, cognitives, psychologiques et sociales de cet état. La définition de l'épilepsie nécessite la survenue d'au moins une crise épileptique [4].
Du point de vue médicolégal, il est important de situer ces actes violents par rapport aux crises épileptiques pour établir la responsabilité pénale car de plus en plus d'épileptiques clament l'irresponsabilité pénale pour cause d'épilepsie. Ceci passe par une connaissance de la clinique de l'épilepsie et par une analyse détaillée des comportements violents et leur contexte de survenue.

Dans un souci de simplification, les termes violence, agressivité ou comportement agressif seront utilisés de façon interchangeable dans cet article.

\section{EPILEPSIE ET COMPORTEMENT AGRESSIF}

La relation entre épilepsie et troubles du comportement est complexe. Sur le plan de la causalité, trois modèles explicatifs sont proposés : 
- L'agressivité est secondaire aux atteintes cérébrales organiques ou aux effets du traitement.

- L'agressivité est un des symptômes psychiques de l'épilepsie.

- Le comportement agressif est la conséquence d'un trouble psychiatrique indépendant de l'épilepsie [5].

Sur le plan clinique descriptif il y a trois formes cliniques d'épilepsies qui pourraient être impliquées dans des comportements violents non intentionnels: les épilepsies généralisées primaires, les épilepsies partielles secondairement généralisées et les épilepsies partielles complexes. Dans les épilepsies partielles ou myocloniques il n'y a pas de troubles de la conscience donc il n'y a pas lieu de supposer l'absence de contrôle sur les actes $[3,6]$.

Les crises toniques, cloniques et atoniques surviennent presque exclusivement chez les enfants présentant un retard mental et sont de durée brève et de nature stéréotypée qu'il est très peu probable qu'ils soient à l'origine d'un comportement violent dirigé [3].

\section{Les comportements agressifs à la phase critique et péri critique:}

Phase critique: L'agressivité au moment des crises épileptiques est rare. Quand elle survient, elle est spontanée, chaotique, mal organisée, sans but et passagère. Le patient n'a pas alors d'antécédents de violence en dehors des crises [1,7]. Il n'a pas été décrit des cas de comportement agressif dirigé contre un individu ou envers un objet survenant au moment des crises ou des cas où ce type de comportement est la seule manifestation de ladite crise [6]. Et si les automatismes critiques pourraient donner lieu à des comportements agressifs, ceux-ci ne doivent pas être élaborés et complexes mais plutôt simples tel pousser quelqu'un par exemple $[1,8]$.

Phase précritique: Une agressivité chez certains épileptiques durant les phases prodromales d'une crise a été décrite, où on note une labilité thymique, une irritabilité, une dépression survenant quelques heures à quelques jours avant une crise [1]. Au cas où le comportement violent coïncide avec le début d'une crise, il s'agira d'un comportement qui n'est pas orienté vers un but ou d'automatismes réactionnels se manifestant par une agressivité dirigée et clairement identifiable survenant au début d'une crise partielle complexe [6].

Phase post critique : Les troubles psychiques constatés au cours de cette période vont de la confusion post critique aux troubles thymiques essentiellement dépressifs et des phénomènes psychotiques (hallucinations visuelles, auditives) ainsi que des troubles de la conscience et une désorientation. La durée de ces phénomènes est influencée par le type et le nombre de crises. La majorité des comportements violents ou agressifs de l'épileptique surviennent au cours de cette phase $[1,3,5,6,8]$. Ils prennent presque exclusivement la forme d'une violence résistive aux tentatives de maîtrise de l'épileptique à la fin d'une crise typique généralisée tonico clonique ou partielle complexe [1, $3,6]$.

La violence survenant au cours des troubles psychotiques post-critiques est généralement orientée vers un but. La psychose post-critique survient chez $10 \%$ des épileptiques, peut durer de quelques heures à plusieurs semaines et a tendance à récidiver. Elle peut évoluer vers un tableau schizophrénique ou affectif. Les symptômes regroupent un délire, des troubles perceptifs, des troubles de la pensée et des troubles thymiques $[1,9]$.
Mais dans ce cas la violence ou l'agressivité n'est pas due à l'épilepsie mais aux conséquences des phénomènes psychotiques délirants post critiques [3].

\section{Les comportements agressifs à la phase inter critique}

Il parait malaisé de tirer des conclusions définitives et catégoriques sur la relation entre le comportement violent inter critique et l'épilepsie tant les données sont contradictoires et mutuellement exclusives [7]. Mais pour la majorité des auteurs la violence inter critique n'est pas liée aux crises épileptiques ni à la maladie épileptique proprement dite et il n'y a pas sur ce plan de différence avec la population générale $[1,6]$. En fait il n'y a pas de preuve scientifiquement établie et dûment documentée attestant que les épileptiques sont plus agressifs que les gens sains ni même que l'épilepsie temporale classiquement connue comme pourvoyeuse de troubles comportementaux présente un risque accru de développement de comportement violent par rapport aux autres formes cliniques de l'épilepsie [3]. Plusieurs facteurs influencent la violence inter critique ou inter ictale, essentiellement les facteurs sociologiques et biologiques. En effet, l'agressivité inter critique est dirigée vers un but (personne ou objet) et elle est influencée par le contexte dans lequel elle survient.

Si l'agitation et la labilité émotionnelle de l'épileptique peuvent précipiter ces actes, si l'intoxication et les effets secondaires du traitement peuvent abaisser le seuil de réaction violente à une provocation ou à une frustration et si l'amnésie de l'acte violent due à la charge émotionnelle qui accompagne ces états sont relevés; ils ne sont ni causaux ni spécifiques [1,3].

Parmi les facteurs incriminés dans l'agressivité inter critique, on retrouve les antécédents de traumatisme crânien, encéphalite, altération des fonctions cognitives, un niveau intellectuel bas, le chômage, un niveau scolaire bas et des conditions socio économiques défavorables [1,3]. Certains attribuent la cause de l'agressivité au syndrome de dyscontrôle épisodique qui ressemble par sa symptomatologie aux crises épileptiques et que certains considèrent comme un type d'épilepsie partielle complexe. Il se manifeste par une incapacité à résister aux impulsions agressives aboutissant à des voies de fait graves ou à la destruction de biens sans lien avec un quelconque facteur de stress psycho social déclenchant [1].

Le trouble dysphorique inter ictal décrit déjà par Kraeplin est aussi évoqué pour expliquer certaines formes de violence chez l'épileptique; dans ce cas la violence est beaucoup plus verbale que physique $[10,11]$.

\section{EPILEPSIE ET CRIMINALITÉ}

Bien que des auteurs célèbres tels que Lombroso et Maudsley aient affirmé au siècle dernier qu'il y avait une relation entre épilepsie et criminalité, cette conception est actuellement totalement remise en cause [8]. L'épilepsie est une pathologie complexe et hétérogène et la criminalité une déviation comportementale multifactorielle et il est quasiment impossible de lier les deux entités dans un rapport de causalité simpliste [12].

Selon Gun, la relation épilepsie- criminalité peut être due soit à une cause : 
- Ictale (la violence est induite par les crises épileptiques) ;

- Accidentelle (il n'y a pas de relation entre les deux phénomènes, l'acte violent survient chez l'épileptique par pure coïncidence) ;

- Psychologique (la violence est la résultante des conséquences psychologiques des crises) ;

- Lésionnelle (les lésions cérébrales ayant causé l'épilepsie ont eux même provoqué des troubles du comportement antisociaux) ;

- Provoquée (les troubles du comportement antisociaux sont marqués par des traumatismes crâniens à répétition, qui eux provoquent l'épilepsie) ;

- Mixte (les causes précédentes s'entremêlent dans a genèse du comportement violent) [12].

La violence chez l'épileptique est corrélée à un certain nombre de facteurs, les uns non spécifiques : sexe masculin, âge inférieur à 40 ans, quotient intellectuel (QI) inférieur à la moyenne, bas niveau socio-économique, mauvais traitements dans l'enfance et d'autres liés à l'épilepsie : début précoce des crises, existence de lésions cérébrales, présence de multiples types de crises $[13,14]$. Les troubles mentaux, notamment délirants, sont également plus nombreux chez les épileptiques et peuvent expliquer la survenue de certains actes violents [14].

Il faut savoir par ailleurs que les données sur la criminalité chez l'épileptique sont rares. Dans une revue de la littérature analysant des meurtres en rapport avec des crises épileptiques survenus de 1880 à 2013, Pandya a retrouvé 50 cas d'homicides commis par des épileptiques. Parmi ces épileptiques, $30 \%$ avaient une épilepsie partielle, $12 \%$ une épilepsie généralisée et $58 \%$ des épilepsies non spécifiées. Parmi les épilepsies partielles, $80 \%$ étaient temporales et $20 \%$ frontales. Il n'y avait pas de relation entre l'épilepsie, la focalisation et l'agressivité (uniquement 22\% de patients ont présenté une agressivité post critique en rapport avec l'épilepsie) [15]. Du point de vue médico légal, $72 \%$ étaient accusés d'homicide volontaire et $22 \%$ d'homicide involontaire. Lors du jugement $62 \%$ étaient déclarés responsable et $38 \%$ irresponsables de leurs actes au moment des faits [15].

Reuber, qui a examiné les dossiers de patients ayant commis des crimes et déclarés irresponsables pour maladie mentale entre 1975 et 2001 en Angleterre et en Écosse, a retrouvé 13 cas $(7,3 \%)$ qui présentaient une épilepsie et qui avaient commis différents crimes ou délits (tentative d'homicide, agression, incendie...). Parmi ces épileptiques, 84,6\% avaient une comorbidité psychiatrique et $61,5 \%$ des offenses étaient commis sous l'effet de l'alcool. Les troubles psychotiques étaient présents dans $52,8 \%$ des cas et $2 / 3$ des actes agressifs ont eu lieu probablement durant la phase post ictale. L'irresponsabilité pénale déclarée lors des jugements ne semble pas être liée aux crises épileptiques et il n'y avait pas de crime commis durant la phase ictale ; la plupart des crimes ont été commis durant la phase post ictale [16].

Fazelae et al. ont mené une étude longitudinale sur une population suédoise entre 1973 et 2009 et dont le but était d'étudier la relation entre épilepsie, traumatisme crânien et crime violent.

Il a été retrouvé que parmi les individus présentant une épilepsie, 4,2\% ont commis un offense ce qui représente un risque plus élevé par rapport à une population contrôle mais cette association disparait lorsque les épileptiques sont comparés à leurs progénitures ce qui fait ressortir de l'étude que après ajustement de l'élément familial confondant il n'y avait pas de relation entre épilepsie et risque accru de violence ou de crime violent [17].
La revue des dossiers médicaux et judiciaires de criminels internés dans un hôpital psychiatrique fermé à Seoul (Corée du Sud) entre octobre 2007 et septembre 2008 a retrouvé sur 761 patients admis ayant commis des infractions ou des crimes $17(2,2 \%)$ étaient épileptiques. Tous les patients avaient une épilepsie partielle et aucun d'eux n'avait commis son crime au moment des crises mais plutôt entre les crises. Parmi ces épileptiques, 8 présentaient en plus une psychose et 7 étaient ivres au moment de l'offense [18].

Les chiffres concernant la prévalence de l'épilepsie dans des prisons sont nuancés. Certains avancent une prévalence d'épilepsie en prison (britanniques et américaines) 2 à 4 fois plus élevées que celle retrouvée en population générale bien que ce taux est similaire à celui retrouvée dans les classes économiques défavorisées d'où vient la majorité des prisonniers $[3,12]$. Par ailleurs une synthèse de sept études cliniques incluant 3000 prisonniers a retrouvé un taux de $1 \%$ d'épileptiques parmi les prisonniers, ce taux était similaire à celui retrouvé en population générale pour des hommes dans la tranche d'âge 25-35 ans [19].

\section{ASPECTS MÉDICO-LÉGAUX}

La question de la responsabilité pénale de l'épileptique est actuellement anecdotique mais elle continue d'être posée et reste une préoccupation clinique car bien que l'épilepsie ne soit pas considérée comme un trouble psychiatrique, les troubles de la conscience accompagnant les crises peuvent être interprétées comme une affection mentale altérant le discernement surtout si un comportement agressif survient au cours des phases ictales ou péri ictales [20,21].

Dans une synthèse d'études sur l'irresponsabilité pénale pour cause de maladie mentale en Grande Bretagne durant la période allant de 1975 à 2001, Mackay a retrouvé 13 cas $(7,3 \%)$ d'épilepsie sur un total de 179 verdicts prononcés par les tribunaux. Les offenses commises vont du meurtre (1 cas) aux tentatives d'assassinat (1 cas), aux coups et blessures volontaires, incendie etc... La majorité des inculpés (12 sur 13 donc $92,3 \%$ des cas) avaient des troubles psychiatriques en plus de la maladie épileptique ce qui augmente considérablement le risque de violence (troubles de la personnalité, dépendance à l'alcool, intoxication alcoolique). Les troubles liés à l'alcool étaient les plus fréquents (intoxication (53,8\%) et sevrage (15,4\%)) [22].

Pour parer à l'éventualité d'utiliser l'épilepsie comme moyen de plaider l'irresponsabilité des critères ont été développés pour une approche méthodique de tout comportement violent survenant chez l'épileptique.

Pour lier les actes violents ou criminels à l'épilepsie ou à la crise épileptique, le prévenu doit réunir les conditions suivantes :

\section{- Antécédents d'épilepsie et de crises épileptiques.}

- Antécédents de comportement anormal apparaissant soudainement, de durée brève et inapproprié aux circonstances.

- Amnésie partielle ou totale de l'événement.

- Témoignage de l'entourage sur l'obscurcissement de la conscience avec mouvements inadaptés, indifférence à l'environnement, errance sans but.

- Absence de motif rationnel du crime.

- Les examens EEG sont compatibles avec la clinique [5].

En 1980 un panel de 18 épileptologues de différents pays s'est réuni aux Etats-Unis sous l'égide de la fondation 
d'épilepsie d'Amérique (EFA) et de l'institut national des troubles neurologiques. Il a analysé, au travers de l'EEG et de la télévision en circuit fermé, les manifestations de 33 crises épileptiques chez 19 patients. Ces patients ont présenté des comportements violents mineurs.

Le rapport a conclu à la rareté des agressions intentionnées au cours des crises épileptiques et la quasi impossibilité de commettre un meurtre ou un homicide involontaire au cours des automatismes psycho moteurs épileptiques.

Le panel a proposé cinq critères pour affirmer qu'un comportement violent est le résultat d'une crise épileptique :

1. Le diagnostic d'épilepsie doit être établi par un neurologue épileptologue.

2. La présence d'automatisme épileptique doit être documentée par les antécédents cliniques, enregistrements vidéo et par l'EEG.

3. La présence d'actes agressifs durant les automatismes doit être vérifiée par des enregistrements vidéos des crises qui doivent être corrélées à l'enregistrement EEG.

4. Les actes violents doivent être une caractéristique habituelle des crises, confirmés par l'histoire de la maladie. L'acte doit survenir soudainement et non en réponse à un stimulus externe. Il doit être bref, simple et limité et être associé à d'autres symptômes des crises épileptiques.

5. Un avis clinique d'un neurologue affirmant la possibilité que l'acte violent fait partie intégrante de la crise épileptique. Le neurologue décrypte l'acte pour affirmer s'il fait partie de la séquence comportementale d'une crise partielle complexe ou s'il est trop élaboré et complexe pout être réalisé par un individu sous l'emprise d'une crise épileptique [3,23].

\section{CONCLUSION}

L'agressivité qui survient chez un épileptique n'est pas le corollaire de la maladie. Au cas où elle pourrait survenir, c'est dans des situations exceptionnelles et pour des comportements violents mineurs. Comme pour la population générale, il y a plusieurs autres facteurs qui induisent la violence ou l'agressivité qu'ils soient psychologiques ou socio économiques. Une connaissance approfondie des syndromes épileptiques ainsi que l'analyse contextuelle détaillée de tout comportement violent est un préalable à une évaluation expertale pour juger de la responsabilité pénale de ces malades.

\section{Messages clés :}

Il n’y a pas de relation entre épilepsie et violence dirigée ou intentionnée.

La violence due à l'épilepsie proprement dite est de nature résistive, désordonnée et sans but; elle survient exclusivement au décours d'une crise généralisée, d'une crise partielle secondairement généralisée ou d'une crise partielle complexe.

La responsabilité pénale de l'épileptique demeure entière sauf pour certains automatismes entraînant toujours des offenses mineures ou des accidents non intentionnels.
Déclaration d'intérêts : l'auteur ne déclare aucun conflit d'intérêt en rapport avec cet article.

\section{RÉFÉRENCES}

1. Marsh L et al. Aggression and violence in patients with epilepsy. Epilepsy \& Behavior, 2000 ; 1:160-168.

2. Ey $H$, Bernard $P$, Prisset $C$. Manuel de psychiatrie. 6èmeéd. Paris : Masson ; 1989. $1166 \mathrm{p}$.

3. Treiman DM. Violence and epilepsy: an approach to expert testimony. In: Rosner R, editor. Principles and practice of forensic psychiatry. 2nd ed. London: Hodder and Arnold ; 2003. p 589-602.

4. Fisher RS, Van Emde Boas W, Blume W, Elger C, Genton P, Lee P, Engel J Jr. et al. Crises épileptiques et épilepsie : définitions proposées par la Ligue internationale contre l'épilepsie et le Bureau international pour l'épilepsie (traduction Pierre Genton). Epilepsies.2005; 17 (3) : 129-32.

5. Faulk M. Basic forensic psychiatry. 2nd ed. London: Blackwell Science; 1994. $380 \mathrm{p}$

6. Treiman DM. Violence and the epilepsy defense. Neurologic clinics, 1999;17:2:245-255.

7. Seward J D. Epilepsy, violence : legal implications. Journal of Police and Criminal Psychology, 1987; 3(1): 35-43.

8. Mellers J D C. Epilepsy. In: David As et al. editors. Lishman's Organic psychiatry. 4th edition. Singapore: Wiley- Blackwell ; 2009. p 309396.

9. Kanner A M. Postictal phenomena in epilepsy. In: Schachter S C et al. Editors. Behavioral aspects of epilepsy, principles and practice. New York. Demos ; 2008. p 105-116.

10. De Toffol B, Philippe Corcia P, Praline J,Mondon K. Impact et enjeux des comorbidités psychiatriques dans les épilepsies. Épilepsies, 2007; 19(4): 231-241.

11. Blumer D P. Interictal dysphoric disorder. In: Schachter S C et al. Editors. Behavioral aspects of epilepsy, principles and practice. New York. Demos ; 2008. p 209-217.

12. Gunn J C. The prevalence of epilepsy among prisoners. Proc. roy. Soc. Med, $1969 ; 62: 60-63$.

13. Chevalier JF, Plas J, Fineyre $F$.Troubles psychiques de l'épilepsie. EMC psychiatrie. 37-219-N-20, 1992, 8 p.

14. Bénézech $M$, Le Bihan $P$ et Bourgeois $M L$. Criminologie et psychiatrie. EncyclMédChir, Psychiatrie, 37-906-A-10, 2002, 15 p.

15. Pandya NS, Vrbancic M, Ladino L D, Téllez-Zenteno J F. Epilepsy and homicide. Neuropsychiatr Dis Treat, 2013; (9): 667-73.

16. Reuber M, Mackay R D. Epileptic automatisms in the criminal courts: 13 cases tried in England and Wales between 1975 and 2001. Epilepsia, $2008 ; 49$ (1):138-45.

17. Fazel $S$, Lichtenstein $P$, Grann $M$, LångströmN. Risk of violent crime in individuals with epilepsy and traumatic brain injury: a 35-year Swedish population study. PLoS Med, 2011;8 (12).

18. Kim J-M, Chu K, Jung K-H, Lee S-T, Choi S-S, Lee S K. Characteristics of epilepsy patients who committed violent crimes: Report from the national forensic hospital. J Epilepsy Res, 2011; 1:13-18.

19. Fazel S, Vassos E, Danesh J. Prevalence of epilepsy in prisoners: systematic review. BMJ, 2002 ; (324) : 1495.

20. Lemesle-Martin M, Smolik H.-J, François-Purssell. I. Aspects médicolégaux et médicosociaux de l'épilepsie. EMC - Neurologie, 17-045-A-60, 2013 ; 10(3) : 1-13.

21. Fenwick P. Epilepsy and the law. BMJ, 1984; 288:1938-1939. 
22. Mackay RD, Reuber M. Epilepsy and the defence of insanityTime for Change? Available at http://www.researchgate. net/profile/Markus_Reuber/publication/263235934_Epilepsy_and _the_defence_of_insanity_-_time_for_a_change/links/02e7e53a3 556fd $989 \mathrm{f000000.}$. Accessed on November 2014.
23. Delgado-Escueta $A \vee$ et al. The nature of aggression during epileptic seizures. Epilepsy \& Behavior, 2002 ; 3: 550-556.

Cet article a été publié dans le « Batna Journal of Medical Sciences » BJMS, l'organe officiel de "l'association de la Recherche Pharmaceutique - Batna »

Le contenu de la Revue est ouvert "Open Access » et permet au lecteur de télécharger, d'utiliser le contenu dans un but personnel ou d'enseignement, sans demander l'autorisation de l'éditeur/auteur.

Avantages à publier dans BJMS :

- Open access : une fois publié, votre article est disponible gratuitement au téléchargement

- Soumission gratuite : pas de frais de soumission, contrairement à la plupart des revues « Open Access ॥

- Possibilité de publier dans 3 langues : français, anglais, arabe

- Qualité de la relecture : des relecteurs/reviewers indépendants géographiquement, respectant l'anonymat, pour garantir la neutralité et la qualité des manuscrits.

Pour plus d'informations, contacter BatnaJMS@gmail.com

ou connectez-vous sur le site de la revue : www.batnajms.com 


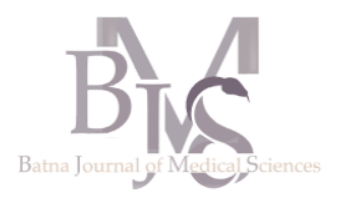

\title{
Actualités sur la vitamine D
}

\author{
Update on vitamin D
}

Malik Djennane ${ }^{1,2}$, Jean-Claude Souberbielle ${ }^{3}$

1. Service de rhumatologie, $\mathrm{CHU}$ de Tizi Ouzou

2.Université Mouloud Mammeri, Tizi Ouzou

3. Service d'Explorations Fonctionnelles, Hôpital Necker-Enfants malades, APHP, Paris, France

\section{Correspondance à : \\ Malik DJENNANE \\ malik.djennane@hotmail.com}

DOI :https://doi.org/10.48087/ BJMSra.2015.2103

\section{Il s'agit d'un article en libre} accès distribué selon les termes de la licence Creative Commons Attribution International License (CC BY 4.0), qui autorise une utilisation, une distribution et une reproduction sans restriction sur tout support ou format, à condition que l'auteur original et la revue soient dûment crédités.

\section{RÉSUMÉ}

Les connaissances sur la vitamine D ont beaucoup progressé ces dernières années. La vitamine $\mathrm{D}$ ne peut plus être considérée comme uniquement nécessaire à la prévention du rachitisme/ostéomalacie. L'évaluation du statut vitaminique D peut être aisément réalisée par le dosage de la 250HD sérique. Toutefois, la plupart des revues récentes sur le sujet suggèrent que les valeurs de référence de la 250HD obtenues dans des populations apparemment en bonne santé sont beaucoup trop basses et que la concentration de 250HD au-dessous de laquelle il existe une insuffisance en vitamine $\mathrm{D}$ se situe entre 50 et $100 \mathrm{nmol} / \mathrm{L}$ (20 et $40 \mathrm{ng} / \mathrm{mL}$ ) avec une franche tendance à cibler des valeurs $>75 \mathrm{nmol} / \mathrm{L}(30 \mathrm{ng} / \mathrm{mL})$. Les supplémentations habituellement recommandées sont insuffisantes pour atteindre ces concentrations.

Mots-clés : vitamine D ; 25 hydroxy vitamine D ; hormone parathyroïdienne ; ostéoporose.

\section{ABSTRACT}

Knowledge of vitamin $\mathrm{D}$ has made great progress in recent years. Vitamin D cannot be considered necessary only in the prevention of rickets/osteomalacia. The assessment of vitamin D status can easily be carried out by the measurement of serum 250HD; However, most of the recent reviews on the subject suggest that the reference values of 250HD obtained in apparently healthy populations are too low and that the concentration of 250HD below which there exist a deficiency in vitamin D between 50 and $100 \mathrm{nmol} / \mathrm{L}$ (20 and 40 $\mathrm{ng} / \mathrm{mL}$ ) with a clear tendency to target values > $75 \mathrm{nmol} / \mathrm{L} \quad(30 \mathrm{ng} / \mathrm{mL})$. The actual recommendations on supplementation seem insufficient to achieve these concentrations.

Keywords: Vitamin D; 25 hydroxy vitamin D; parathyroid hormone; osteoporosis

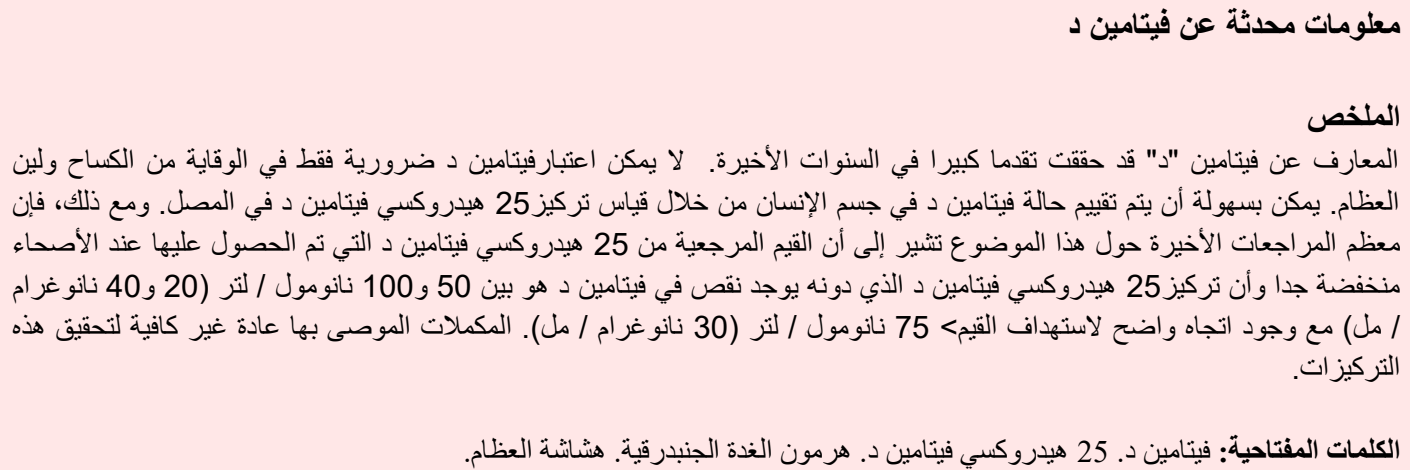

\section{INTRODUCTION}

La vitamine $\mathrm{D}$ est très importante pour la croissance et la santé osseuse. Le terme vitamine est inapproprié pour la vitamine $\mathrm{D}$ qui doit être plutôt considérée comme une pro hormone. La peau peut la synthétiser à partir du 7-dehydrocholesterol sous l'effet de certains rayonnements UVB (longueur d'onde entre 290 et $315 \mathrm{~nm}$ ) (1).

La vitamine D existe sous deux formes, la vitamine D3 (cholécalciférol) qui est la molécule synthétisée par la peau sous l'influence des UVB ou retrouvée dans les rares sources alimentaires animales (poissons gras en particulier), et la vitamine D2 (ergocalciférol) qui est la vitamine $\mathrm{D}$ des plantes.

La vitamine D (D2 ou D3) est transportée dans le sang par une protéine porteuse, la vitamin $D$ binding protein (DBP), jusqu'au foie pour y subir une première transformation. Dans le foie, la vitamine $D$ est hydroxylée sur le carbone 25 par l'enzyme 25-hydroxylase pour former la 250HD (25-hydroxy-cholécalciférol ou calcidiol). Cette hydroxylation n'est pas régulée, plus la quantité de vitamine D synthétisée ou ingérée est importante, plus la quantité de $250 \mathrm{H}$ D formée est grande. Ceci permet son utilisation en pratique courante 
pour évaluer les réserves d'un sujet en vitamine D.

La vitamine D est liposoluble et une partie est stockée dans le tissu adipeux. Ainsi, le stockage sera plus important chez les sujets obèses que chez les maigres, et la production hépatique de $250 H D$ en sera diminuée [2]. La 250H D circule dans le sang, transportée par la DPB, avec une demi-vie de l'ordre de trois à quatre semaines. Cette hydroxylation est catalysée par une autre enzyme à cytochrome $\mathrm{P} 450$, la 1-alpha-hydroxylase (CYP27B1), présente sur la membrane interne des mitochondries [3].

Une seconde hydroxylation a ensuite lieu au niveau rénal sous l'action de l'enzyme 1-alpha-hydroxylase, pour donner la forme active de la vitamine $\mathrm{D}$ : la 1,25(OH)2D (1,25 dihydroxy-cholécalciférol ou calcitriol). Cette hydroxylation est étroitement régulée et stimulée par la parathormone (PTH), par une hypophosphorémie ou de faibles apports alimentaires en calcium ou en phosphate, et par l'IGF. Elle est inhibée par le FGF-23 et une hyperphosphatémie et par la 1,25(OH) 2 D elle même. La 1,25 (OH) 2 D est le métabolite actif de la vitamine $\mathrm{D}$, sa demi-vie dans le sérum est de quatre heures [3].

La figure 1 ci-dessous schématise le différents processus du métabolisme de la vitamine $\mathrm{D}$.

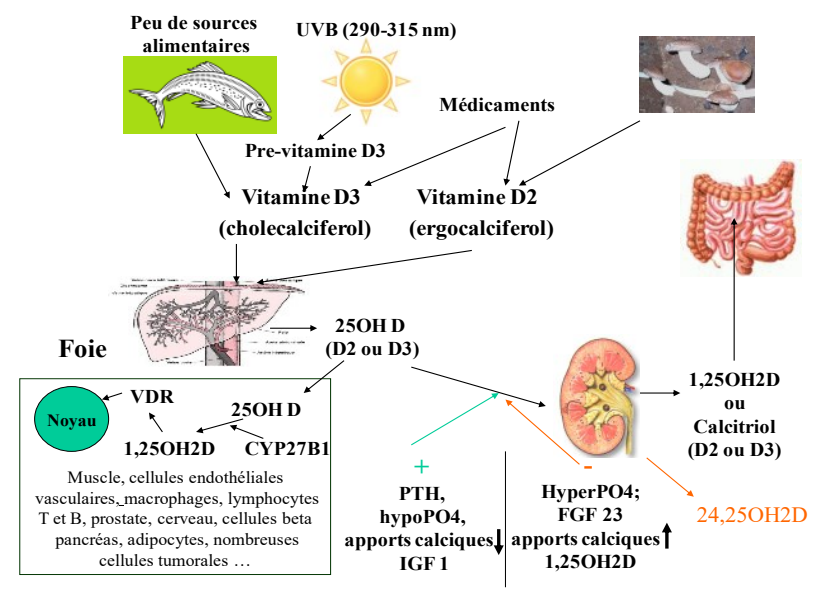

Figure 1 : Métabolisme de la vitamine D

\section{MÉCANISMES D’ACTION DE LA VITAMINE D}

La 1,25(OH) 2D peut exercer :

- Des actions « endocrines" : la 1,25(OH) 2D produite par le rein est transportée par voie systémique jusqu'à ses tissus cibles.

- Des actions "autocrines": de nombreux tissus expriment la 1-alpha-hydroxylase ainsi que le VDR et la 24hydroxylase. La $25(\mathrm{OH})$ D pénètre dans ces tissus et y est hydroxylée en $1,25(\mathrm{OH}) 2 \mathrm{D}$ ou elle agit localement au niveau cellulaire, l'excès étant métabolisé en composé inactif sous l'action de la 24-hydroxylase.

\section{Actions endocrines de la vitamine D}

Dans la cellule cible, la $1,25(\mathrm{OH}) 2$ D se lie au récepteur cytosolique, le VDR, présent dans de nombreux tissus. Ce complexe VDR-1,25(OH) 2 D se lie lui même au récepteur de l'acide rétinoïque (RXR) au niveau du noyau de la cellule. Le complexe RXR-VDR-1,25(OH) 2 D se lie enfin à l'ADN en des sites spécifiques de réponse à la vitamine $\mathrm{D}$ [2].

\section{Actions génomiques intracrines de la vitamine D}

Le second mode d'action met en évidence une production intracrine de $1,25(\mathrm{OH})_{2} \mathrm{D}$, qui ne participe pas au métabolisme phosphocalcique mais qui a bel et bien des effets métaboliques voire cliniques. Ce mode d'action concernerait de nombreux tissus, et la $1,25(\mathrm{OH})_{2} \mathrm{D}$ contrôlerait l'expression de plusieurs gènes (300 à 1000), qui régulent la prolifération des cellules saines et cancéreuses à savoir leur différenciation, leur apoptose et leur angiogenèse , la régulation également des gènes intervenant dans l'immunomodulation [3].

\section{EFFETS DE LA 1,25(OH $)_{2}$ D}

Effets classiques de la vitamine $D$ : Maintien de l'homéostasie phosphocalcique: L'os contient 99\% dU calcium, le calcium circulant constitue une petite part du calcium de l'organisme. Pour que la minéralisation osseuse soit optimale, le produit phosphocalcique doit rester stable. La phosphatémie est régulée, d'une part par le rein et d'autre part par le FGF-23 qui diminue la réabsorption tubulaire proximale des phosphates et inhibe la synthèse de la $1,25(\mathrm{OH})_{2} \mathrm{D}$.

Au niveau intestinal : L'absorption intestinale du calcium se fait principalement au niveau du duodénum et de la portion proximale du jéjunum. Dans la cellule intestinale, la $1,25(\mathrm{OH})_{2} \mathrm{D}$ induit la synthèse d'un canal calcique, via la proteine TRPV6. Ce processus actif est prépondérant lorsque les apports calciques ou phosphorés sont faibles ou dans des conditions physiologiques (croissance, grossesse) ou pathologiques (granulomatoses, hyperparathyroïdies) ou la concentration plasmatique de $1,25(\mathrm{OH})_{2} \mathrm{D}$ est élevée, permettant ainsi d'augmenter la fraction de calcium et de phosphore absorbée par rapport à la quantité ingérée. Ceci va assurer un climat minéral optimal pour l'os et sa minéralisation [4].

Au niveau rénal: L'effet principal de la 1,25(OH) $)_{2} \mathrm{D}$ au niveau du tubule contourné proximal est le rétrocontrôle négatif sur sa propre synthèse avec une inhibition de l'activité 1-alpha-hydroxylase. La $1,25(\mathrm{OH})_{2} \mathrm{D}$ augmente la réabsorption du calcium au niveau des cellules du tubule distal. Son effet stimulant sur la réabsorption tubulaire des phosphates est secondaire à l'inhibition de la sécrétion de PTH [5].

Au niveau osseux : La vitamine D contrôle la transcription, la différenciation et la minéralisation des ostéoblastes. La $1,25(\mathrm{OH})_{2} \mathrm{D}$ contrôle également l'expression de certaines protéines comme le collagène et l'ostéocalcine qui possèdent des VDRE ( récepteurs à la vitamine D). La vitamine D stimule les ostéoclastes, libérant ainsi les minéraux contenus dans la matrice osseuse. Pour cela, elle est d'abord reconnue par les ostéoclastes qui surexpriment alors le ligand RANK (Receptor Activator of Nuclear Factor-kBLigand). Ce ligand se fixe ensuite sur son récepteur RANK situé sur les pré-ostéoclastes et l'union du récepteur à son ligand engendre la transformation du pré-ostéoclaste en ostéoclaste mature. Les ostéoclastes déplacent alors le calcium et le phosphore contenus dans l'os vers la circulation sanguine et augmentent ainsi le produit phosphocalcique [6].

$\mathrm{Au}$ niveau des glandes parathyroïdiennes: Il existe une expression des récepteurs sensibles au calcium capables de détecter les variations de la calcémie, une baisse de cette dernière entrainera l'augmentation de la synthèse et de la sécrétion de la PTH. La $1,25(\mathrm{OH})_{2} \mathrm{D}$ exerce un rétrocontrôle négatif sur les glandes parathyroïdes en inhibant la synthèse et la sécrétion de PTH. Elle exerce également un rétrocontrôle sur la croissance des cellules parathyroïdes [7]. 


\section{MÉCANISMES DE RÉGULATION}

La production rénale de 1,25 $(\mathrm{OH})_{2} \mathrm{D}$ est finement régulée et stimulée par la parathormone, mais également les états d'hypocalcémie et d'hypophosphatémie. La production rénale de 1,25(OH $)_{2}$ D est inhibée par le Fibroblast Growth Factor 23 (FGF23), une hyperphosphatémie et la $1,25(\mathrm{OH})_{2} \mathrm{D}$ elle même. Le FGF 23 est secrété par l'os. Il effectue ainsi un rétrocontrôle négatif en diminuant la synthèse de 1,25 $(\mathrm{OH})_{2} \mathrm{D}$, et en diminuant directement l'absorption phosphocalcique intestinale et la réabsorption rénale.

La $1,25(\mathrm{OH})_{2} \mathrm{D}$ diminue sa propre production dans un phénomène de rétrocontrôle négatif. Enfin, il existe une voie d'activation de la vitamine D via une enzyme, la 24 hydroxylase (CYP-24) qui induit la production de composés inactifs $\left(24,25(\mathrm{OH})_{2}\right.$ vitamine $\mathrm{D}, 1,24,25(\mathrm{OH})_{3}$ vitamine D) transformés ensuite en acide calcitroique inactif [3].

\section{VALEURS DE RÉFÉRENCE DE LA 25(OH)D SÉRIQUE}

La mesure de la $1,25(\mathrm{OH})_{2} \mathrm{D}$ n'est pas appropriée pour évaluer le statut vitaminique D. C'est la 25(OH)D qui représente le stock de vitamine $\mathrm{D}$ de l'organisme, qui doit être mesurée pour savoir si un individu a ou non un déficit en vitamine D. Ce constat est désormais consensuel [2]. Les valeurs de $25(\mathrm{OH}) \mathrm{D}$ sont exprimées en nmol/L (égales à $\mathrm{ng} / \mathrm{ml} \times 2,5)$.

De nombreux scientifiques et experts discutent sur le fait que les valeurs de référence de 25(OH)D sont inadaptées et basses et que les différentes supplémentations recommandées sont insuffisantes, ce qui a conduit à une nouvelle réflexion pour l'établissement de ces valeurs de référence [6].

Pour établir des valeurs de référence d'une variable biologique, on doit la mesurer chez un grand nombre de personnes en bonne santé, supposés être représentatifs de la population de référence, puis on calcule un intervalle de référence correspondant en général à $95 \%$ de la population, et on établit à partir de cet intervalle les limites basses et hautes de la variable biologique.

On ne peut pratiquer de la sorte pour la vitamine D car, chez les sujets en bonne santé, la valeur de $25(\mathrm{OH}) \mathrm{D}$ est variable et dépendra de la population étudiée, de la saison du prélèvement, de la latitude et de l'altitude du lieu de résidence des sujets, de leur âge et de leur phototype. Devant ce fait établi, il n'est donc pas aisé d'établir une population de référence représentative de la population générale [8]. La véritable définition de l'insuffisance, qui se profile actuellement, est la concentration de $25(\mathrm{OH}) \mathrm{D}$ en dessous de laquelle, dans une population en bonne santé, la PTH augmente statistiquement.

Lips [9] a proposé de définir l'insuffisance en vitamine D par des concentrations de 25(OH)D pour lesquelles il peut exister des effets délétères pour la santé et en particulier pour l'os (hyperparathyroïdie secondaire et ou augmentation du remodelage osseux). JC Souberbielle et al en 2008 ont trouvé plusieurs approches utilisées pour définir les concentrations de $25(\mathrm{OH}) \mathrm{D}$ associées à un statut vitaminique $\mathrm{D}$ optimal et par conséquent pour définir l'insuffisance en vitamine D [3]. Ils proposent de les séparer en plusieurs catégories.

\section{Définir l'insuffisance en vitamine $D$ par le seuil au dessous duquel il existe une hyperparathyroïdie secondaire.}

L'hyperparathyroïdie secondaire est une augmentation de la sécrétion de la PTH afin de compenser une tendance à la baisse de la calcémie ionisée. On peut de fait observer une PTH sérique dans les limites de la normale et avoir une hyperparathyroïdie secondaire, d'autant plus que, pour une technique de dosage de PTH donnée, les valeurs de référence peuvent être significativement différentes en fonction de la population de référence recrutée [10].

\section{vitamine $D$ et la fréquence de certaines maladies}

De nombreuses études observationnelles ont pu montrer une relation positive entre la concentration basse de $25(\mathrm{OH}) \mathrm{D}$ et la fréquence de certaines maladies (diabète, polyarthrite rhumatoïde, cancers, sclérose en plaques, tuberculose, événements cardiovasculaires etc....). Généralement, les sujets dans le «quantile» supérieur de 25(OH)D (correspondant à une concentration de $25(\mathrm{OH}) \mathrm{D}>30 \mathrm{ng} / \mathrm{ml}$ ) ont un risque relatif inférieur à ceux dans le "quantile» inférieur [11].

Ces études observationnelles ont évidemment une place inférieure «moins d'évidence-based medicine» que les études interventionnelles. Elles ne permettent pas d'établir un lien de causalité entre le déficit en vitamine $\mathrm{D}$ et les différentes maladies évoquées.

\section{ÉTUDES D'INTERVENTION MONTRANT DES EFFETS POSITIFS DE LA VITAMINE D}

Des études interventionnelles ont montré une réduction du risque relatif de développer certaines pathologies après supplémentation vitaminique D. Nous citerons que certaines :

- Réduction du risque de fractures pour un seuil de 25(OH)D de 75 à $100 \mathrm{nmol} / \mathrm{l}$ soit 30 à $40 \mathrm{ng} / \mathrm{ml}$ [12].

- Réduction du risque de chutes pour un seuil de 25(OH)D supérieur à $60 \mathrm{nmol} / \mathrm{l}$ [13].

- Réduction du risque de cancer [14].

- Réduction de la pression artérielle après trois mois d'exposition à des UVB pendant lesquels la 25(OH)D est passée de 58 à $151 \mathrm{nmol} / \mathrm{L}(32,2$ à $60,4 \mathrm{ng} / \mathrm{ml})$, ou après $1200 \mathrm{mg}$ de calcium et $800 \mathrm{UI} / \mathrm{J}$ de vitamine D3 pendant deux mois pendant lesquels la 25(OH)D est passée de 25,7 à $64,8 \mathrm{nmol} / \mathrm{l}$ (10,3 à 25,9 ng/ml) [15].

- Amélioration significative de la fonction des cellules beta de langerhans et élévation moindre de l'Hémoglobine glyquée A1c chez des patients à haut risque de diabète de type 2 après 16 semaines de supplémentation par cholécalciférol (2000 UI/J) (seuil de 25(OH) D passant de 24 à $31 \mathrm{ng} / \mathrm{ml}$ ). [16].

Plutôt que le terme de valeurs normales ou de référence, on utilisera maintenant plus souvent la notion de valeurs «souhaitables», la valeur seuil de 250HD définissant l'insuffisance en vitamine D (c'est à dire la concentration minimale qu'il faut avoir) étant de :

$\checkmark$ Pour Lips [9] dans sa revue de 2001: $50 \mathrm{nmol} / \mathrm{L}$ (20 $\mathrm{ng} / \mathrm{ml}$ )

$\checkmark$ Pour Holick [2] dans sa revue de 2002 : $50 \mathrm{nmol} / \mathrm{l}$ (20 $\mathrm{ng} / \mathrm{ml}$ ) afin d'éviter l'hyperparathyroïdie secondaire et $75 \mathrm{nmol} / \mathrm{L}$ ( $30 \mathrm{ng} / \mathrm{ml}$ ) pour profiter d'autres effets de la vitamine D.

$\checkmark$ Pour Zittermann [17] dans sa revue de 2003 :100 $\mathrm{nmol} / \mathrm{l}(40 \mathrm{ng} / \mathrm{ml})$.

\section{FACTEURS DE RISQUE ET DÉTERMINANTS DE L'HYPOVITAMINOSE D}

\section{Localisation géographique}

La quantité d'Ultraviolets (UV) qui arrive à la surface de la terre dépend de nombreux facteurs dont l'angle des rayons 
UVB par rapport à la couche d'ozone et la distance à parcourir à travers l'atmosphère. L'intensité des rayons ultraviolets varie selon la latitude, elle est maximale au niveau de l'équateur et s'atténue avec l'augmentation de la latitude [2].

\section{L'altitude}

Elle joue aussi un rôle sur la concentration en vitamine D, l'ensoleillement est de plus forte intensité en montagne qu'en plaine. Les rayons UVB sont en effet atténués en traversant l'atmosphère : ils seront donc moins atténués s'ils ont une faible distance à parcourir dans l'atmosphère. De plus, ces UVB sont atténués par les particules en suspension dans les zones polluées : ces zones se situent surtout autour des grandes villes et plutôt en plaine, ce qui va majorer la faible intensité d'UVB au sol [18].

\section{La saison}

La synthèse de vitamine $\mathrm{D}$ varie de la même manière selon les saisons. Pendant les mois d'hiver, les radiations sont moins intenses et de plus courte durée, ce qui explique pourquoi la synthèse de vitamine $\mathrm{D}$ est plus difficile pendant les mois d'hiver dans beaucoup de pays de l'hémisphère Nord. Dans l'étude française de Guillemant $J$ et al à Chantilly $\left(49^{\circ}\right.$ de latitude Nord), la variation saisonnière est évidente, le taux de 25(OH)D est à son taux le plus bas en hiver par rapport à l'été [18]. Dans une autre étude de Docio S et al, la variation saisonnière est évidente, à Cantabria (Nord de l'Espagne $\left(43^{\circ}\right.$ de latitude nord), le taux de 25(OH)D est plus bas en hiver qu'en été [19]. Dans une étude en Hongrie de Bakos et al, les UVB à Budapest, latitude $47^{\circ}$ Nord, étaient suffisants de mars à octobre pour la photosynthèse, alors que de novembre à février, ils étaient très insuffisants, même après ajustement selon l'habillement lié à la température et le temps passé à l'extérieur [20].

\section{L'heure d'exposition}

Elle doit aussi être prise en compte dans la production de vitamine D. Ainsi à Boston $\left(42^{\circ} \mathrm{N}\right)$, la photosynthèse de précholécalciférol est efficace de 7 h00 à $17 \mathrm{~h} 00$ en période d'été, alors qu'au printemps et en automne elle n'est efficace que de 10 h00 à 15 h00 [19].

\section{Phototype}

La pigmentation de la peau est une cause principale dans la carence en vitamine $\mathrm{D}$ parce que la mélanine absorbe les rayons UVB. Cette pigmentation joue un rôle d'écran solaire naturel et l'augmentation de la pigmentation cutanée peut réduire la synthèse de vitamine $\mathrm{D}$ sous l'effet des UVB d'une manière aussi efficace qu'un écran solaire. Les afroaméricains sont les plus carencés en vitamine $\mathrm{D}$ et les hommes blancs le sont moins, avec toutefois une prévalence de 34,9\% [20]. De même, dans l'étude de G Guardia de 2008, la proportion des personnes présentant une carence en vitamine D était significativement plus élevée chez les personnes de couleur de peau noire, que celle des personnes de couleur de peau claire [21].

\section{Age}

Les sujets âgés ont en général des taux de 25(OH)D plus faibles que les sujets jeunes d'une même région. Ceci a été démontré dans l'étude NHANES III où l'âge moyen augmentait significativement au fur et à mesure des quartiles descendants de 25(OH)D. La peau des personnes âgées contient moins de 7-déhydro-cholestérol, et à partir de lui, synthétise moins de pré-vitamine D3 [18].

\section{Le stade pubertaire}

La puberté constitue également une période à risque de carence en vitamine $\mathrm{D}$. En effet, une étude a montré que près de $25 \%$ des adolescents âgés de 10 à 15 ans sont en carence de 25(OH)D (taux inférieur à $10 \mathrm{ng} / \mathrm{ml}$ ) en période pré hivernale. Le principal facteur de cette hypovitaminose est la majoration des besoins en vitamine $\mathrm{D}$ en rapport avec l'accroissement de la demande en calcium du squelette [22]. Il est important de souligner qu'une carence pubertaire induira un pic de masse osseuse plus bas que celui des adolescents non carencés, ce qui peut avoir pour conséquences une ostéoporose précoce.

\section{Sexe}

Les études sont contradictoires. Il semblerait que les filles aient des taux plus bas que les garçons sans que ce résultat ne soit consensuel [23].

\section{Le mode de vie}

Le type vestimentaire et religieux est un facteur influençant le risque de carence de vitamine D. Le travail du Dr Belaïd a montré la forte prévalence de carence chez les patientes portant un vêtement couvrant. En effet, 99\% des 96 patientes étudiées avaient un taux de vitamine inférieur à $21,2 \mathrm{ng} / \mathrm{ml}$ [23]. En Turquie (Istanbul $41^{\circ} \mathrm{N}$ ), une étude réalisée chez des femmes de 14 à 44 ans suggère que l'exposition des mains et de la face permet de synthétiser la vitamine D mais pas de manière suffisante pour éliminer une carence [24].

\section{La pratique d'une activité sportive}

L'exercice physique modéré favorise l'accumulation de la masse osseuse, plus particulièrement pendant la période pubertaire. La maturité du squelette et le pic de masse osseuse, atteints vers l'âge de 20 ans, dépendent de facteurs génétiques, hormonaux, environnementaux et de l'activité physique. Les forces mécaniques, en stimulant la formation ostéoblastique, favoriseraient la production locale de facteurs de croissance [25].

\section{La protection solaire}

Elle est le sujet de nombreuses campagnes de prévention en France et dans le reste du monde. Cette prévention conseille d'utiliser des crèmes solaires, de porter des vêtements longs, un chapeau et d'éviter l'exposition aux heures les plus dangereuses. Les crèmes solaires fonctionnent comme des barrières bloquant les rayons UV. Cependant de nombreuses études n'ont pas pu mettre en évidence un lien entre l'utilisation de crème solaire et l'hypovitaminose [26].

\section{L'indice de masse corporelle}

La vitamine D étant liposoluble, une partie est stockée dans le tissu adipeux. Ainsi le stockage sera d'autant plus important qua la masse grasse est grande pour un individu donné, et la production hépatique de $25(\mathrm{OH}) \mathrm{D}$ en sera diminuée. Les sujets obèses ou en surpoids ont des concentrations sériques 
de vitamine D plus basses que les sujets maigres, ceci a été démontré dans l'étude américaine NHANES III [27].

\section{Les pathologies chroniques}

Les pathologies chroniques induisant une hypovitaminose D sont les suivantes :

$\checkmark$ Insuffisance rénale chronique entrainant un défaut de transformation de la 25(OH)D en $1,25(\mathrm{OH})_{2} \mathrm{D}$

$\checkmark$ Malabsorption : maladie de Crohn, maladie cœliaque, maladie de Whipple, pancréatite chronique, mucoviscidose, obstruction biliaire.

$\checkmark$ La chirurgie gastrique (By-pass).

$\checkmark$ L'insuffisance hépatique entrainant un défaut d'hydroxylation de la vitamine $\mathrm{D}$ en position 25.

$\checkmark$ Syndrome néphrotique entrainant une fuite de 250HD dans les urines.

$\checkmark$ Séquelles de brulures étendues (diminution de la capacité de la peau à synthétiser la vitamine D3).

$\checkmark$ Tumeurs osseuses sécrétant le FGF 23 en excès.

$\checkmark$ Hyperparathyroïdie primaire, qui engendre un excès de transformation de 25(OH)D en $1,25(\mathrm{OH})_{2} \mathrm{D}$, d'où une concentration basse de 25(OH)D.

$\checkmark$ Granulomatoses, sarcoïdose, tuberculose et certains lymphomes, via les macrophages qui transforment également excessivement la $250 \mathrm{HD}$ en $1,25(\mathrm{OH})_{2} \mathrm{D}$.

$\checkmark$ Hyperthyroïdie, responsable d'une accélération du métabolisme de la 25(OH)D.

$\checkmark$ Maladies génétiques : défaut de production de la $1,25(\mathrm{OH})_{2} \mathrm{D}$ (rachitisme pseudo-carentiel de type I) ; résistance à la $1,25(\mathrm{OH})_{2} \mathrm{D}$ (rachitisme pseudo- carentiel de type II par mutation/délétion récepteur VDR) ; tubulopathies héréditaires avec perte urinaire de phosphates.

\section{Traitements médicamenteux}

Certains médicaments modifient le métabolisme de la vitamine D comme les anticonvulsivants, les glucocorticoïdes, les immunosuppresseurs en prévention des rejets de greffes et des traitements antirétroviraux. Ces médicaments augmentent la transformation de la 25(OH)D et de $1,25(\mathrm{OH})_{2} \mathrm{D}$ en composés inactifs en position 24 favorisant ainsi l'hypovitaminose D [2].

\section{Statut physiologique}

Chez les nourrissons, l'allaitement maternel exclusif constitue un risque de carence en vitamine D. Il est donc nécessaire de les supplémenter. Les laits infantiles étant supplémentés en vitamine $\mathrm{D}$, les autres nourrissons seront moins exposés aux carences. Chez la femme enceinte, une supplémentation vitaminique D systématique doit être mise en place au 3 ème trimestre de grossesse [21].

\section{CONCLUSION}

Les connaissances sur la vitamine D ont beaucoup progressé ces dernières années. La vitamine $\mathrm{D}$ ne peut plus être considérée que comme nécessaire à la prévention des rachitismes/ostéomalacies. L'évaluation du statut vitaminique $\mathrm{D}$ peut être aisément réalisée par le dosage de la 250HD sérique. Toutefois, la plupart des revues récentes sur le sujet suggèrent que les valeurs de référence de la 250HD sont beaucoup trop basses et que la concentration de 250HD au-dessous de laquelle il existe une insuffisance en vitamine D se situe entre 50 et $100 \mathrm{nmol} / \mathrm{l}$ (20 et $40 \mathrm{ng} / \mathrm{ml}$ ). Dans ce contexte, les supplémentations habituellement recommandées risquent d'être insuffisantes pour atteindre ces concentrations.

\section{Déclaration d'intérêts :}

M.D : l'auteur ne déclare aucun conflit d'intérêt en rapport avec cet article.

JC.S : orateur pour DiaSorin, Roche Diagnostics et Abbott diagnostics.

\section{RÉFÉRENCES}

1. Holick M. Vitamin D : the underappreciated D-lightful hormone that is important for skeletal and cellular health. Curr Opin Endocrinol Diabetes 2002; $9: 87-98$.

2. Holick MF. Vitamin D deficiency. N Engl J Med. 2007; 357:266-81

3. Souberbielle JC, Prié $D$, Courbe baisse $M$, et al. Uptade on vitamin D and evaluation of vitamin D status. Ann Endocrinol (paris) 2008; 69:501-10

4. Nemere I, Schwartz Z, Pedrozo H, et al. Identification of a membrane receptor for 1,25 dihydroxyvitamin D3 which mediates rapid activation of protein kinase C. J Bone Miner Res 1998; 13:1353-9

5. Scanlon v. Sanders T. 2006. Essentials of anatomy and physiology. Fifth edition. USA: F. A. Davis Company. 603 p.

6. Jurutka PW, Bartik L, Whitfield GK et al. Vitamin D receptor : Key Roles in bone mineral, pathophysiology, molecular mechanism of action and novel nutritional ligands. J Bone Miner Res 2007; 22 (suppl 2): V2-V10

7. Cantley LK, Russel J, Lettieri D, et al. 1,25-Dihydroxyvitamin D3 suppresses parathyroid hormone secretion from bovine parathyroid cells in tissue culture. Endocrinology 1985; 117:2114-9

8. McKenna M, Freaney R.Secondary hyperparathyroidism in the elderly: means to defining hypovitaminosis D. Osteoporos Int 1998; 8 (suppl 2): S3-S6

9. Lips P. Vitamin D deficiency and secondary hyperparathyroidism in the elderly: consequences for bone loss and fractures and therapeutic implications. Endocr Rev 2001; 22: 477-501

10. Glendenning $P$, Gutteridge $D H$, Retallack et al. High prevalence of normal total calcium and intact PTH in 60 patients with proven primary hyperparathyroidism: a challenge to current diagnostic criteria. Aust N Z J Med 1998; 28:173-8

11. Hypponen E, Power C. Vitamin D status and glucose homeostasis in the 1958 British birth cohort: the role of obesity. Diabetes Care 2006; 29:2244-6

12. Bischoff-Ferrari HA, Willett WC, Wong JB, et al. Fracture prevention with vitamin $D$ supplementation: a meta-analysis of randomized controlled trials.JAMA 2005; 293:2257-64

13. Bischoff-Ferrari HA, Dawson-Hughes B, Steahelin HB, et al. Fall prevention with supplemental and active forms of vitamin $D$ : a meta-analysis of randomized controlled trials. BMJ 2009; 339-369

14. Lappe JM, Travers-Gustafson D, Davies KM et al. Vitamin D and calcium supplementation reduces cancer risk: Results of a randomized trial. Am J Clin Nutr 2007; 85:1586-91

15. Pfeifer M, Begerow B, Minne HV, et al. Effects of a short-term vitamin D (3) and calcium supplementation on blood pressure and parathyroid hormone levels in ederly women. J Clin Endocrinol Metab 2001; 86: 1633-7

16. Mitri J, Dawson-Hughes B, Hu FB et al. Effects of vitamin D and calcium supplementation on pancreatic $b$ cell function, insulin sensivity, and glycemia in adults at high risk of diabetes: the calcium and vitamin D for diabetes Mellitus randomized controlled trial. Am J Clin Nutr 2011; 94:486-94 
17. Zittermann $A$. Vitamin $D$ in preventive medicine: are we ignoring the evidence? Br J Nutrition. 2003; 89: 552-572

18. Guillemant J, Allemandou A, Cabrol S et al. Statut vitaminique D de I'adolescent: variations saisonnières et effets d'une supplémentation hivernale par la vitamine D3. Arch Pédiatr.1998; $5: 1211-5$

19. Docio S, Riancho JA, Perez A et al. Seasonal deficiency of vitamin D in children. J Bone Miner Res. 1998; 13:544-8

20. Bakos $J$ and Miko P.Vitamin D forming effectiveness of ultraviolet radiation from sunlight in different months in Budapest, Hungary. Orv Hetil. 2007; 148: 319-25

21. Guardia G, Parikh N, Eskridge T, Phillips E, Divine G and Sudhaker Rao D. Prevalence of vitamin D depletion among subjects seeking advice on osteoporosis a five year cross sectional study with public health implications. Osteoporos Int. 2008;19:13-9.

22. Duhamel JF, Zeghoud F, Sempe M et al. Prophylaxie de la carence en vitamine $D$ chez l'adolescent et le préadolescent. Étude interventionnelle multicentrique sur les effets biologiques d'un apport répété de 100.000 UI de vitamine D. Arch.Pediatr. 2000; 7:148-53
23. Hill TR, Cotter AA, Mitchell S, et al. Vitamin D status and parathyroid hormone relationship in adolescents its association with bone health parameters: analysis of the Northen Ireland young Heart's Project. Osteoporosis Int 2010; 21:695-700

24. Alagol $F$, Shihadeh $Y$, Boztepe $H$, et al. Sunlight exposure and vitamin D deficiency in Turkish women. J Endocrinol Invest. 2000; 23:173-7.

25. Lehtonen-Veromaa $M$, Möttönen $T$, Svedström $E$, Hakola $P$, Heinonen OJ, Viikari J. Physical activity and bone mineral acquisition in peripubertal girls. Scand J Med Sci Sports. 2000; 10:236-43

26. Scarlett WL. Ultraviolet radiation: sun exposure, tanning beds, and vitamin D levels. What you need to know and how to decrease the risk of skin cancer. J Am Osteopath Assoc.2003; 103:371-5.

27. Looker AC, Pfeiffer CM, Lacher DA et al. Serum 25-hydroxyvitamin D status of the US population: 1988-1994 compared with 2000-2004. Am J Clin Nutr 2008; 88:1519-27

Cet article a été publié dans le « Batna Journal of Medical Sciences » BJMS, l'organe officiel de "l'association de la Recherche Pharmaceutique - Batna»

Le contenu de la Revue est ouvert "Open Access » et permet au lecteur de télécharger, d'utiliser le contenu dans un but personnel ou d'enseignement, sans demander l'autorisation de l'éditeur/auteur.

Avantages à publier dans BJMS :

- Open access : une fois publié, votre article est disponible gratuitement au téléchargement

- Soumission gratuite : pas de frais de soumission, contrairement à la plupart des revues « Open Access ॥

- Possibilité de publier dans 3 langues : français, anglais, arabe

- Qualité de la relecture : des relecteurs/reviewers indépendants géographiquement, respectant l'anonymat, pour garantir la neutralité et la qualité des manuscrits.

Pour plus d'informations, contacter BatnaJMS@gmail.com

ou connectez-vous sur le site de la revue : www.batnajms.com 


\section{Bis}

Service de physiologie et des explorations fonctionnelles, CHU Benflis touhami, Batna, Algérie.

\section{Correspondance à :}

Salah FERHI

salahferhi17@gmail.com

DOI :https://doi.org/10.48087/ BJMSra.2015.2104

Il s'agit d'un article en libre accès distribué selon les termes de la licence Creative Commons Attribution International License (CC BY 4.0), qui autorise une utilisation, une distribution et une reproduction sans restriction sur tout support ou format, à condition que l'auteur original et la revue soient dûment crédités.

\title{
Diagnostic de la Broncho-pneumopathie Chronique Obstructive asymptomatique par spirométrie
}

\section{Diagnosis of asymptomatic chronic obstructive pulmonary disease using spirometry}

\author{
Salah Ferhi, Boubakeur Kermiche, Naouel Chibout, Mohamed Ridha Guedjati
}

\section{RÉSUMÉ}

Le dépistage de la Broncho-pneumopathie chronique obstructive (BPCO) asymptomatique reste, sur le plan scientifique, un sujet de controverse. Pas d'études contrôlées prouvant en chiffre un bénéfice notablement supérieur aux dépenses allouées pour une telle mesure préventive, du moins jusqu'à 2008. Cette réalité scientifique n'a pas empêché beaucoup de spécialistes dans le domaine de la BPCO, d'une façon individuelle ou collective à travers des sociétés savantes nationales ou internationales de préconiser son dépistage, du moins dans des populations à risques, tabagiques surtout. Une attitude de bon sens, devant une pathologie qui constitue un réel problème de santé publique dans les pays industrialisés comme dans les pays en voie de développement, de par sa prévalence, sa morbidité, sa mortalité et ses dépenses élevées et pour laquelle, sont réunis dans une large mesure les critères de dépistage d'une pathologie chronique adoptés par l'OMS. Ce dépistage se fait par la mesure du souffle (spirométrie). Idéalement par des mini spiromètres en consultation de médecine générale. Une confirmation reste nécessaire après test de bronchodilatation qui se fait habituellement au niveau des laboratoires ou des unités d'exploration fonctionnelle respiratoire. Des efforts de sensibilisation adressés à la population générale aux responsables politiques et aux professionnels de santé notamment aux médecins généralistes, sur la gravité de la BPCO et l'élargissement de l'utilisation de la spirométrie, à différents niveaux de la consultation, semblent être la plateforme d'un dépistage efficace.

Mots-clés : Broncho-pneumopathie obstructive chronique, spirométrie, dépistage.

\begin{abstract}
Screening for asymptomatic chronic obstructive pulmonary disease (COPD) is still, on a scientific way, a controversial topic. Few controlled studies have numerically proven a benefit that is superior to expenses of such a preventive measure, until 2008. This scientific reality did not prevent some specialists in the fields of COPD, in an individual or collective manner, through their medical national or international societies to prescribe the screening for COPD, especially in at-risk populations, such as smoking people. A common-sense attitude, when dealing with a pathology that is a real public health problem in developed as well as developing countries, due to its high prevalence, morbidities, health expenditures, and for which screening is highly recommended by the World Health Organization. This screening can be done using the measure of breath pressure (spirometry), ideally by using small spirometers that can be available at general practitioner's' out patients setting. A validation is needed after a bronchodilation test, which is performed in laboratories or lung function test units. Efforts of awareness rising addressed to the general population and to political officials, and especially to general practitioners, on the severity of COPD and the utility of spirometry, are the first step towards insuring an efficacious screening.
\end{abstract}

Keywords: chronic obstructive pulmonary disease, spirometry, screening.

\section{الكثف المبكر عن مرض الانسداد الرئوي المزمن

لا يزال الكثف المبكر عن مرض الانسداد الرئوي المزمن من الناحية العلمية، يثير الكثير من الجدل. ولا يوجد دليل الإليل للفائدة

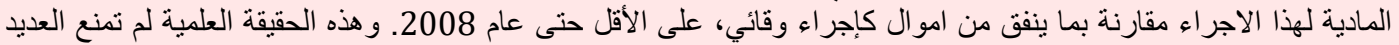

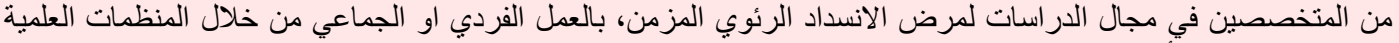

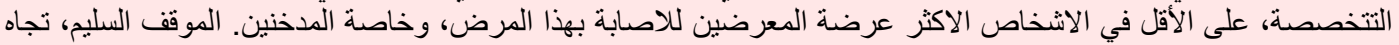

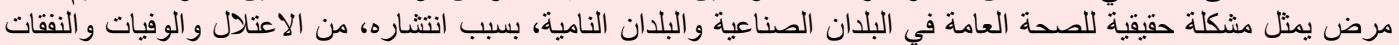

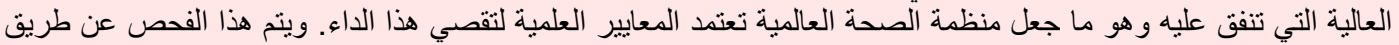

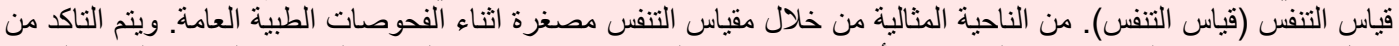

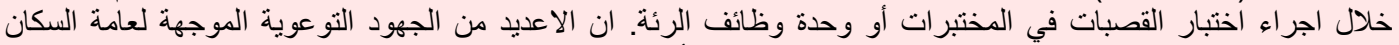

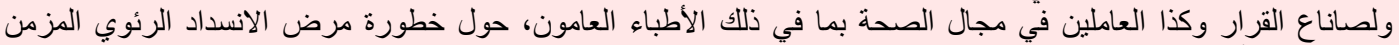

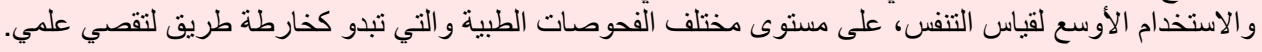

$$
\text { الكلمات المفتاحية: مرض الانسداد الرئوي المزمن، قياس التنفس، الكثف المبكر }
$$

Ferhi Kermiche B CHibout N, et al. Diagnostic de la bronchopneumopathie chronique obstructive spirométrie. Bathe par Sci 2015;2(1):13-18. https://doi.org/10.48087/ BJMSra.2015.2104 


\section{INTRODUCTION}

La broncho-pneumopathie chronique obstructive (BPCO), regroupe en fait, deux pathologies respiratoires chroniques; l'emphysème pulmonaire et la bronchite chronique obstructive. Ces deux pathologies donnent, sur le plan fonctionnel, essentiellement, un trouble ventilatoire de nature obstructive (TVO), d'évolution progressive, insidieuse, vers l'insuffisance respiratoire chronique. Malgré que cette maladie constitue un problème de santé publique majeur, sa méconnaissance de la population générale ou encore des professionnelles de santé est d'actualité et reste un handicap pour sa prise en charge efficace. Le caractère purement fonctionnel de sa définition (TVO persistant après test de broncho-dilatation) est à la base de son diagnostic par l'exploration de la fonction ventilatoire ou spirométrie. Le retard dans l'apparition des symptômes respiratoires, notamment la dyspnée alors que l'obstruction bronchique est déjà très évoluée justifie en partie son diagnostic le plus précocement possible.

\section{BCPO : PATHOLOGIE GRAVE ET MÉCONNUE}

Pour mieux connaitre la BPCO nous avons choisi de présenter et sans modification la définition approuvée par la société de pneumologie de langue française (SPLF) dans la mise à jour en 2009 de ses recommandations pour la prise en charge de cette affection [1], et qui est fondée sur celles des sociétés savantes de pneumologie des Etats-Unis, du Canada, de Grande-Bretagne et de France, qui a considéré que la BPCO est une maladie respiratoire chronique définie par une obstruction permanente et progressive des voies aériennes. La cause la plus fréquente est le tabagisme. Cette obstruction est causée par l'association, variable selon les patients, d'une diminution $\mathrm{du}$ calibre des bronchioles $\mathrm{du}$ fait de modifications anatomiques (remodelage) et d'une destruction des alvéoles pulmonaires (emphysème). Il s'y associe une réponse inflammatoire pulmonaire anormale à des toxiques inhalées (tabac, polluants...). Les maladies suivantes ne font pas partie de la BPCO : l'asthme, les dilatations des bronches et l'atteinte pulmonaire de la mucoviscidose. La présence de dilatations des bronches est possible dans la BPCO mais elles ne constituent pas alors l'élément central de la pathologie.

L'évolution de la BPCO est marquée par :

- Un déclin accéléré de la fonction respiratoire ;

- Un risque d'exacerbations pouvant mettre en jeu le pronostic vital ;

- Un risque de handicap avec réduction de l'activité quotidienne notamment lié à la dyspnée.

L'évolution de la maladie peut aboutir à une insuffisance respiratoire chronique. Il existe fréquemment des Comorbidités qui doivent être recherchées car elles aggravent les symptômes et le pronostic. (Comorbidités : présence d'une ou plusieurs affections ou maladies chroniques coexistant avec la BPCO sans préjuger d'un lien causal). Le diagnostic de trouble ventilatoire obstructif (TVO) de la BPCO est fondé sur la spirométrie avec la mesure du volume expiratoire maximal à la première seconde (VEMS) et de la capacité vitale forcée (CVF). Il est défini par un rapport VEMS/CVF $<70 \%$ après administration d'un bronchodilatateur $[1,2]$.

Pour un éclaircissement sur la gravité de la BPCO, les constats épidémiologiques qui vont suivre, et qui portent sur sa prévalence, sa morbidité et sa mortalité dans quelques pays représentatifs et à l'échelle mondiale, prouvent sans le moindre doute, la réalité fâcheuse d'une pathologie fréquente, handicapante, mortelle, et qui reste sous estimée, sous diagnostiquée et sous traitée [3]. Cependant il faut noter la diversité dans les chiffres présentés dans ces exemples et dans la littérature d'une façon générale [4], notamment ceux de la prévalence. Cette diversité s'explique premièrement par la nature de la population étudiée (générale ou ciblé), secondairement par la méthode utilisée, à savoir :

1. Les questionnaires standardisés (source de surestimation);

2. L'expression de symptômes par le patient (source de sous estimation) :

3. La spirométrie (méthode de référence, concordante avec la définition de la BPCO), surtout si la diminution physiologique du Rapport VEMS/CV avec l'âge est prise en considération [5].

Cependant, l'utilisation de cette dernière méthode est limitée dans les études épidémiologiques suite aux difficultés pratiques de sa réalisation à grande échelle. Ainsi, et à titre d'exemples :

En Algérie : dans la wilaya d'Alger, la prévalence de la BPCO est de $4,9 \%$ pour l'ensemble de la population, elle est rare avant l'âge de 40 ans $(0,1 \%)$ et atteint 9,2\% chez les plus âgés. Chez ces derniers. Le tabagisme majore cette prévalence qui atteint $31,5 \%$ chez les fumeurs, $14,6 \%$ chez les exfumeurs et $2,5 \%$ chez les non-fumeurs. Elle augmente avec l'âge. La prévalence des différents stades de sévérité est respectivement de $6 \%, 2,7 \%, 0,3 \%$ et $0,2 \%$. [6].

Au Vietnam : elle est de $13,5 \%$ dans une population à risque (fumeurs $>10 \mathrm{P} / \mathrm{A}$ et ex-fumeurs dont l'âge $>40$ ans) [7].

En France, le travail de synthèse des données épidémiologiques françaises effectué par C. Fuhrman et M.C.Delmas pour le groupe épidémiologie et recherche clinique de la SPLF motionne que la prévalence est entre 5 et $10 \%$ au delà de 45 ans. La mortalité est de 16.500 cas en 2006. Le taux hospitalisation pour exacerbation est compris entre 69000 et 112000, ce taux a augmenté entre 1998 et 2006 [8].

Aux États-Unis, la prévalence est de 7,14\%. Le taux de diagnostic est de $37 \%$, soit $2 / 3$ des malades sont méconnus. La forme légère à modérée (principale cible de dépistage) est 10 fois supérieure à celle de la forme sévère. Les symptômes sont de mauvais facteurs prédictifs de l'obstruction bronchique, absents chez $20 \%$ des sujets dont le VEMS est < à $50 \%$ de la théorie. Au Etats-Unis toujours, l'augmentation de la mortalité par BPCO est rapide par rapport aux autres causes, elle est de l'ordre de $+163 \%$ entre 1965 et 1998 (Figure 1).

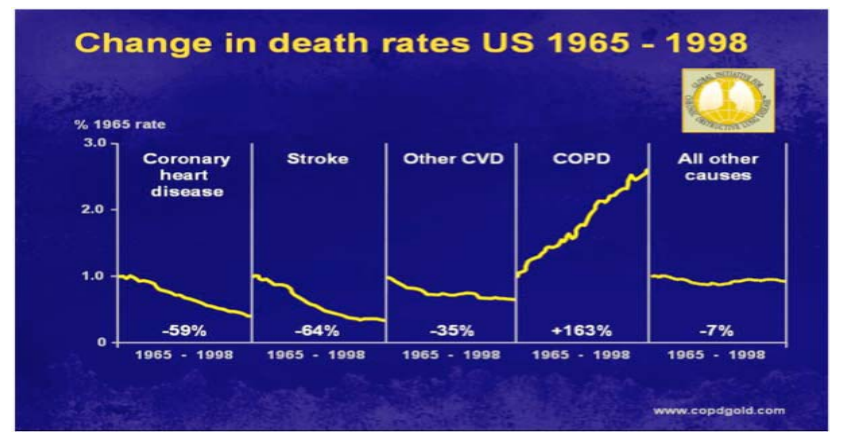

Figure 1 : Causes de mortalité au Etats-Unis entre 1965-1998

Au Canada, Toronto - Plus d'une personne sur quatre devrait développer une broncho-pneumopathie chronique 
obstructive (BPCO) au cours de sa vie, d'après l'analyse des données d'une étude de population canadienne, publiée dans un numéro spécial de l'Européen Respira tory Society du Lancet début septembre. Au total, 579466 cas de BPCO ont été diagnostiqués parmi environ 13 millions d'habitants de la province de l'Ontario de plus de 35 ans et de moins de 80 ans ne souffrant pas de BPCO sur une durée de14 ans. Globalement, l'incidence de la BPCO à l'âge de 80 ans était de $27,6 \%$. Le risque était plus élevé chez les hommes $(29,7 \%)$ que chez les femmes $(25,6 \%)$ et en fonction du statut socioéconomique $(32,1 \%$ pour les statuts les plus bas vs $23 \%$ pour les plus élevés). En outre, Les personnes vivant en milieu rural étaient plus à risque $(32,4 \%)$ que les habitants de zones urbaines $(26,7 \%)$ [10].

A l'échelle mondiale et dans la projection de l'OMS de 2020, la BPCO devrait être la première pathologie respiratoire mortelle. Elle occupera la $3^{\text {ème }}$ place parmi le reste des pathologies, après sa 6ème place en 1990 (figure 2). Selon L'OMS toujours la BPCO est l'une des principales causes d'hospitalisations et l'une des maladies chroniques les plus coûteuses.

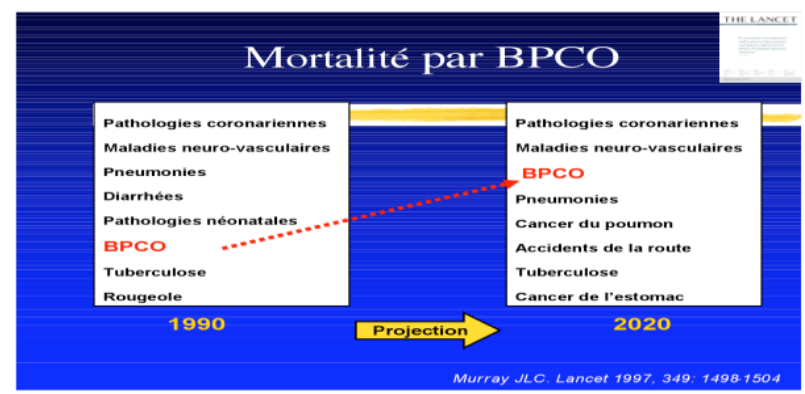

Figure 2 : Projection 2020 de l'OMS des causes de mortalité (vers la $3^{\text {ème }}$ place pour la BPCO).

L'étude de l'histoire naturelle de la BPCO, met en évidence ses différents aspects évolutifs de gravité croissante à savoir la dégradation progressive de la fonction respiratoire, le retard dans l'apparition des symptômes, qui sont source de son installation insidieuse et donc d'un sous diagnostic. A un stade avancé du déclin du VEMS, la maladie devient symptomatique avec surtout l'installation progressive de la dyspnée qui constitue dès lors un véritable handicap limitant la capacité à l'exercice et l'activité physique quotidienne. Le pronostic est majoré par les exacerbations source d'hospitalisation et par la Comorbidité, source d'aggravation des symptômes. L'aboutissement se fait vers l'insuffisance respiratoire chronique.

Pour la dégradation progressive de la fonction respiratoire (ventilatoire), Fletcher et coll, ont suivi la progression du VEMS (paramètre le plus représentatif de la fonction ventilatoire) en fonction du temps, dans deux populations : fumeurs et non fumeurs, et ont ainsi démontré que (figure 3) :

1. une diminution physiologique de la fonction ventilatoire est notée chez les non fumeurs et les fumeurs non sensibles au tabac (la sensibilité est en termes de fonction respiratoire) estimé à $25 \%$ entre 25 et 75 ans, c'est-à-dire durant 50 ans d'évolution.

2. Chez les fumeurs sensibles à la fumée, une dégradation importante de la fonction ventilatoire s'installe progressivement. Une réduction de $25 \%$ du VEMS est déjà présente à l'âge de 45 ans (courbe rouge). Le VEMS s'abaisse à $50 \%$ avant 60 ans et l'invalidité respiratoire s'installe à 65 ans, correspondant à une réduction du VEMS de $70 \%$ (toujours par rapport à sa valeur à 25 ans).
3. Le bénéfice en termes de fonction respiratoire par sevrage tabagique est surtout important lorsque la limitation du débit aérien reste modérée (début de la courbe en tiré), c'està-dire à une étape précoce de la maladie. Le sevrage tardif à 65 ans (début de la flèche noire), est toujours nécessaire, il limitera plutôt la dégradation rapide.

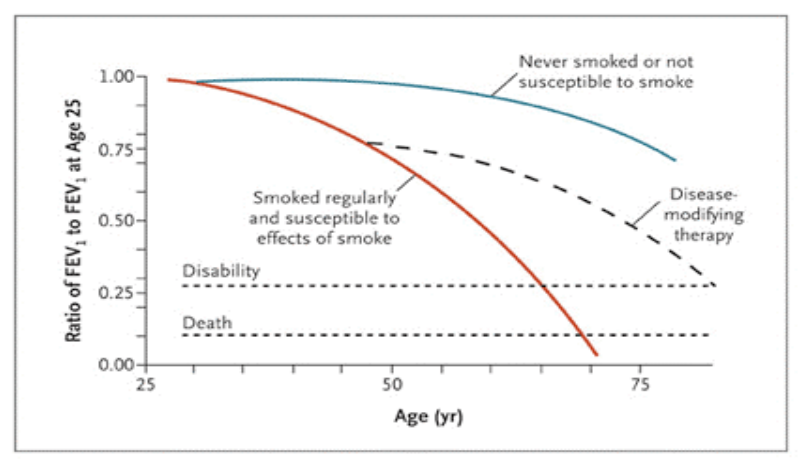

Figure 3 : Courbes de diminution du VEMS, chez les fumeurs, exfumeurs et les non fumeurs, d'après Fletcher et Peto 1977.

Pour le retard dans l'apparition des symptômes de la BPCO, la figure suivante montre l'absence de symptômes respiratoires en présence d'une importante anomalie spirométrique (réduction du VEMS d'environ 40\% par rapport à sa valeur théorique). C'est le cas par exemple d'une étude faite au Etats-Unis qui révèle que $20 \%$ des sujets dont le VEMS est < à $50 \%$ ne présentent pas de symptômes respiratoires. Donc un dépistage parait initialement justifié par l'existence de cette étape préclinique (figure 4).

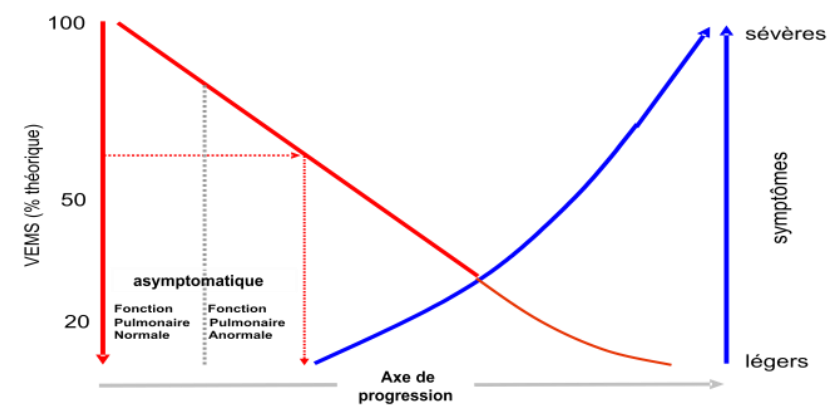

Figure 4: Rapport entre la diminution du VEMS et l'apparition de symptômes respiratoires. Sutherland et Cherniack, N E J Med 2004.

Pour la Comorbidité de la maladie, les figures suivantes (figure 5 et 6), représentent des exemples, présentés au congrès de l'européen respiratory society (ERS), qui s'est déroulé en septembre 2012 à Viennes :

\section{BPCO-COMORBIDITÉS}

L'insuffisance cardiaque gauche est très souvent associée à des anomalies fonctionnelles respiratoires

- Étude prospective réalisée chez 63 sujets, dont 43 femmes, âgés de 77 ans en moyenne $30 \%$ de fumeu

Résultats :

Anomalies fonctio

$(88 \%)$ de leffectif

Déficit ventilatoire restrictif : $16 \%$

(14\%

Hypoxémie $(60 \%)$, anomalie du transfert du CO $(82 \%)$

$\rightarrow$ Chez l'ensemble des patients, ces anomalies fonctionnelles respiratoires n'étaient pas diagnostiquées avant leur participation à cette étude
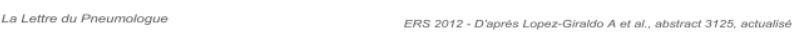

Figure 5 : BPCO et insuffisance cardiaque gauche. Le TVO est retrouvé pure dans $30 \%$ des cas et dans $14 \%$ associé à un Trouble (Déficit) Ventilatoire Restrictif. 


\section{BPCO}

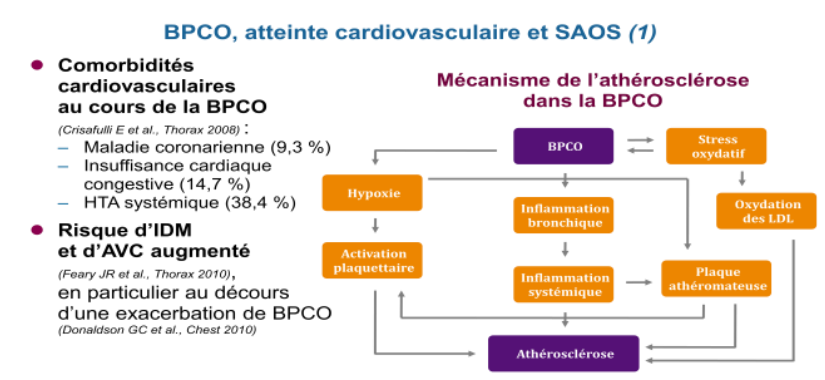

Figure 6 : BPCO et comorbidités cardio-vasculaires. Noter bien l'importance de l'HTA présente dans $38,4 \%$ des cas.

Pour l'insuffisance respiratoire chronique, elle constitue l'aboutissement terminal de la maladie.

$\mathrm{Au}$ total, tous les aspects de gravité sus cités, semblent justifier de sérieux efforts pour la prise en charge de cette pathologie, à commencer par son diagnostic, le plus précocement possible.

\section{INTÉRÊTS POTENTIELS DU DIAGNOSTIC PRÉCOCE}

Il existe une controverse entre les sociétés savantes dans la scène internationale sur l'indication du dépistage de la BPCO chez le sujet à risque en l'absence de symptomatologie respiratoire. C'est ainsi qu'un intéressant article écrit par des spécialistes français en la matière [11], à été publié dans la revue des maladies respiratoires, porte parole de la SPLF en juin 2008 intitulé: Faut-il dépister la BPCO dans la population? Cet article constitue une réponse analytique, scientifique de l'argumentation avancée par les services de prévention des Etats-Unis, paru dans un article publié au début de l'année 2008 dans les Annals of Internal Medecine, dans lequel cet organisme recommande fortement de ne pas dépister la BPCO, mais bizarrement, son analyse de la littérature selon les règles de la médecine fondée sur les preuves n'a touchée qu'un seul critère d'évaluation du dépistage, qui est le niveau de diminution des exacerbations. Cependant d'autres critères d'évaluation dont la pertinence n'est plus à démonter pour cette pathologie comme la dyspnée, la capacité d'exercice, l'activité quotidienne, la qualité de vie, la comorbidité et la mortalité, n'ont pas été inclus par faute de données dans la littérature. Ce qui reflète un grade $\mathrm{D}^{*}$ de cette recommandation négative, concordant plutôt avec : il ne faut probablement (voir peut-être) pas faire le dépistage $\left(^{*}:\right.$ le système d'évaluation scientifique de type grade permet de pondérer la force des conclusions selon la solidité scientifique des arguments qui les sous-tendent). L'Americain College of Chest Physicians en 2007 de son coté a émis une recommandation négative. Le National Health Service anglais réserve le dépistage aux patients à risque et symptomatiques. De l'autre coté l'initiative GOLD en 2011 [12], est pour le dépistage par spirométrie, chez tout sujet qui présente une histoire personnelle d'exposition aux facteurs de risque de la maladie, en particulier la fumée de tabac, les fumées domestiques ou industrielles, et l'exposition professionnelle aux poussières ou à des dérivés chimiques, qu'il soit symptomatique ou non, surtout si son âge est supérieur à 40 ans. La SPLF en 2003 et l'ATS/ERS en 2004 sont pratiquement du même avis.

Pour l'indiquer, une opération de dépistage l’OMS en 1971 a adopté pour son compte un ensemble de Critères (critères de Wilson), une fois réunis un test de dépistage peu être appliqué pour une pathologie chronique. Il s'agit essentiellement des quatre Critères suivants [Critères de WILSON (OMS 71) [13] :

1. La maladie concernée est fréquente et crée un problème de santé individuel ou collectif.

2. Il existe un test de dépistage fiable, sensible, spécifique, peu couteux et non invasif applicable à la population concernée.

3. Le dépistage à un stade précoce apporte un bénéfice pour le sujet atteint (amélioration de l'état physique ou de la qualité de vie ou de la survie).

4. La société peut assurer la prise en charge du dépistage et le traitement des individus dépistés.

On remarque bien que le critère (1) concerne la pathologie, (2) concerne le test de dépistage, et (3), (4) concernent les résultats de l'opération de dépistage.

En application des Critères de WILSON à la BPCO :

- le critère (1) est prouvé.

- le critère (2) s'applique sur la spirométrie, (question qui sera développée en « diagnostic précoce par spirométrie »).

- pour (3) et (4) le manque de preuves scientifiques en faveur du dépistage pour une population asymptomatique ne semble pas empêcher son indication du moins de la part de quelques organismes scientifiques de renommée. Motivé très probablement par la gravité de la maladie (voir supra) et par les intérêts potentiels d'une telle opération, à savoir :

1. La motivation et l'aide pour le sevrage tabagique : quelques résultats d'étude sont en faveur d'une amélioration des chances de la réussite d'un sevrage tabagique une fois un Trouble ventilatoire obstructif détecté par spirométrie est annoncé au patient. L'arrêt de la consommation tabagique permet la limitation de la dégradation rapide de la fonction respiratoire.

2. La motivation pour reprendre, commencer ou maintenir une activité physique sportive régulière (contre argument : cette mesure préventive est largement indiquée pour lutter contre la sédentarité facteur de risque cardiovasculaire).

3. Le renforcement de l'indication d'une vaccination antigrippale, antipneumococcique (contre argument: cette indication est déjà codifiée et conditionné par l'âge).

4. L'obtention du profil fonctionnel ventilatoire de référence, est d'intérêt pour un suivi longitudinal des populations accessibles, notamment en milieu professionnel à risque.

\section{DIAGNOSTIC PRÉCOCE PAR SPIROMÉTRIE}

Le diagnostic précoce peut se faire, selon la littérature, par un questionnaire, qui reste de spécificité et de sensibilité moyennes, plus ou moins associé à une spirométrie miniaturisée de spécificité et de sensibilité meilleure, reste que, la confirmation passe obligatoirement par une spirométrie conventionnelle avant et après bronchodilatation.

À travers l'expérience du personnel médical et paramédical du service de physiologie et des explorations fonctionnelles, CHU Batna, guidé par la connaissance théorique, 
principalement tirée des recommandations de l'ATS/ERS de 2005 traitant la standardisation des explorations fonctionnelles respiratoires [14], la conduite de l'examen spirométrique se fait comme suit :

S'assurer initialement de la qualité de la mesure par l'introduction des valeurs des paramètres atmosphériques (température: automatiquement, pression atmosphérique (en $\mathrm{mmHg}$ ) et humidité relative : manuellement), puis étalonnage du spiromètre par une seringue certifiée de 3 litres.

Interrogatoire et examen clinique, en partie pour identifier une contre-indication absolue ou relative à l'exploration: Pneumothorax en cours. Ponction ou biopsie pleurale récente. Hémoptysie en cours. Crise d'asthme. État cardiovasculaire instable. Infection respiratoire. Prise d'un bronchodilateur $(<6 \mathrm{~h}$ si d'action rapide, $<12 \mathrm{~h}$ si action prolongée), prendre en compte un traitement corticoïde en cours. Prise d'une cigarette $(<1 \mathrm{~h})$.

Prise et introduction des caractéristiques biométriques (taille et poids), en plus de l'introduction de l'âge et du sexe, avec choix des valeurs de référence les plus proches de notre population locale [15].

Installation du patient, en position assise ou debout (la position assise est préférée pour le confort du patient mais surtout pour sa sécurité). Desserrer une ceinture, une cravate. Cou en extension. Maintien d'un dentier.

Présentation et mise en place du matériel d'exploration (le patient tient le capteur, filtre antibactérien agencé, pince nez posé). Exécution de quelques cycles ventilatoires calmes, pour que le patient s'assure que l'exploration n'est pas douloureuse, n'est pas vraiment gênante.

Explication du but de l'exploration et des étapes des manœuvres ventilatoires nécessaires. Ceci avec un langage compréhensible, avec des mots adaptés au niveau d'éducation du patient. Une démonstration est le plus souvent nécessaire pour l'amélioration de la compréhension et la coopération par la suite lors de l'exécution des manœuvres.

Deux manœuvres sont à exécuter : capacité vitale lente (CVL) et capacité vitale forcée (CVF) :

Manœuvre de la CVL : se déroule comme suit :

1. Plusieurs cycles ventilatoires courants.

2. Inspiration complète.

3. Expiration lente et complète.

En obtient alors la courbe de variation lente du volume pulmonaire mobilisable par rapport au temps (Figure 7). Le paramètre principal mesuré est la CVL.

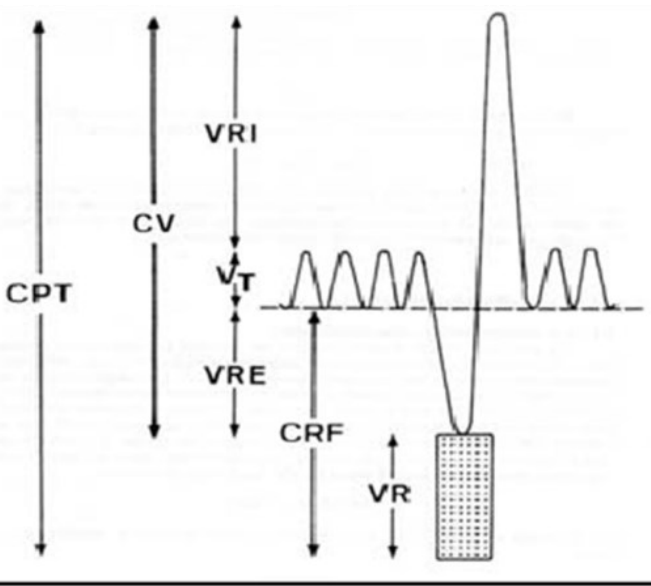

Manœuvre de la CVF : se déroule comme suit :

1. Après plusieurs cycles ventilatoires courants.

2. Inspiration complète.

3. Expiration d'emblée maximale, la plus forte et la plus rapide et la plus longtemps possible (complète).

4. Inspiration profonde.

En obtient alors la courbe débit/volume (Figure 8).

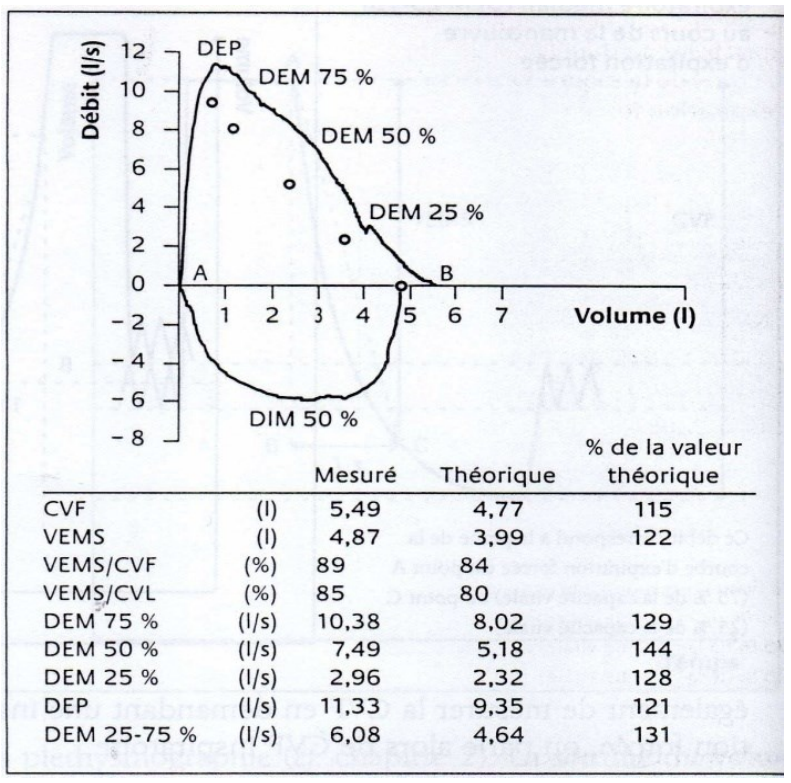

Figure 8. Courbe Débit/Volume.

Les paramètres ainsi obtenus sont : la CVF, le VEMS, le débit de pointe (DEP), le débit expiratoire maximal moyen entre 25 et $75 \%$ de la CV (DEM 25-75\%, ou DEMM25-75\%) ainsi que les débits maximaux instantanés, essentiellement à 25 et à $50 \%$ de la CVF.

La répétition des manœuvres est obligatoire à la recherche de 3 manœuvres valide (acceptables) est maximales (reproductible, répétable) surtout pour la manœuvre de CVF. La manœuvre de la CVF est valide lorsqu'elle commence rapidement, ne présente pas d'artéfacts (toux à la première seconde...), et qu'elle est complète. Elle est reproductible lorsque la différence est $<150 \mathrm{ml}$ entre les 2 plus grands VEMS et entre les deux plus grandes CVF.

Test de réversibilité : nécessaire au diagnostic de la BOCO. C'est une spirométrie faite 15 minutes après introduction, habituellement de bronchodilatateur de courte durée d'action (prise de 400 microgrammes de salbutamol avec une chambre d'inhalation. 100 microgrammes chaque 30 secondes).

L'interprétation des résultats est recommandation dépendante, notamment pour le Trouble ventilatoire obstructif [16]. Au niveau de notre laboratoire elle se base essentiellement sur les recommandations ATS/ERS 2005, GOLD 2011 et SAPP 2012.

Le TVO correspond à un VEMS/CVF $<70 \%$ selon GOLD2011. Il correspond à un VEMS/CV (la meilleure entre CVF ou CVL) < LIN selon l'ATS/ERS2005.

L'évaluation de la sévérité du TVO se base uniquement sur l'aspect spirométrique qui utilise la valeur du VEMS mesurée par rapport à la valeur prédite suivant les recommandations GOLD :

Figure 7. Courbe Volume/temps lente 
- Léger VEMS $\geq 80 \%$ de la valeur prédite

- Modéré $80 \%>$ VEMS $\geq 50 \%$ de la valeur prédite.

- Sévère $50 \%>$ VEMS $\geq 30 \%$ de la valeur prédite.

- Très sévère VEMS $<30 \%$ de la valeur prédite.

La réversibilité du TVO est jugée :

1. significative sur la base d'une amélioration du VEMS et/ou la CVF de plus de $12 \%$ et plus de $200 \mathrm{ml}$ en valeur absolue par rapport à la valeur initiale (ATS/ERS 2005). L'amélioration en pourcentage se calcule comme suit : (valeur post-bronchodilatation - valeur prébronchodilatation) / valeur pré-bronchodilatation.

2. Elle sera totale (complète) s'il y a disparition du TVO, c'est-à-dire une normalisation du rapport VEMS/CVF, qui devient $>70 \%$ pour la définition GOLD 2011 ou > LIN pour la définition ATS/ERS 2005.

3. La persistance du TVO après broncho dilatation détermine une réversibilité incomplète est définie par conséquent une BPCO.

Les valeurs de référence (prédites) utilisées sont celles de l'ERS 1993 par manque de valeurs de référence locales.

\section{CONCLUSION}

L'élargissement de la diffusion du maximum d'information sur la BPCO, reste à notre sens une attitude de base, nécessaire, qui doit être menée continuellement, et adressée, par les acteurs de santé impliqués dans la prise en charge directe de cette maladie, à tous les autres acteurs de santé, et de la, au reste de la population générale. Une éventuelle prise de conscience sur la gravité de la BPCO, permet sans doute, un investissement réel dans sa prévention primaire, en luttant contre ces facteurs de risque, et dans sa prévention secondaire par l'utilisation de la spirométrie, du moins dans les populations à risque.

Déclaration d'intérêts : l'ensemble des auteurs ne déclare aucun conflit d'intérêt en rapport avec cet article.

\section{RÉFÉRENCES}

1. Société de pneumologie de langue française. Recommandation pour la Pratique Clinique. Prise en charge de la BPCO. Mise à jour 2009. Rev Mal Respir 2010 ; 27 : 522-548.

2. Société algérienne de pneumo-phtisiologie. Recommandation pour les explorations fonctionnelles respiratoires 2008. Non publié

3. Jebrak G. Recommandations et prise en charge de la BPCO en France : les recommandations sur la prise en charge de la BPCO ne sont pas suivies dans la vraie vie ! Rev Mal Respir 2010 ; 27 : 11-18.

4. Numéro thématique. La bronchopneumopathie chronique obstructive (BPCO). BEH 2007 : 27-28.

5. Lettre ouverte aux membres du comité GOLD. Rev Mal Respir 2010 ; 27 : 1003-7.

6. Khelafi R, Aissanou A, Tarsift S, Skander F. Epidémiologie de la bronchopneumopathie chronique obstructive dans la wilaya d'Alger. Rev Mal Respir $2011 ; 28$ : 32-40.

7. Duong-Quy S, Hua-Huy T, Mai-Huu-Thnah B, et al. Détection précoce de la bronchopneumopathie chronique obstructif posttabagique au Viet Nam. Rev Mal Respir 2009 ; 26 :267-74.

8. Fuhrman C, Delmas MC. Epidémiologie descriptive de la bronchopneumopathie chronique obstructive (BPCO). Rev Mal Respir 2010 ; $27: 160-168$.

9. Fishberg S, Motamed S, Janssens J-P. Pratique et interprétation de la spirométrie au cabinet du médecin de premier recours. Rev Med Suisse 2009; 5 :1882-1889

10. Gershon SA, Warner L. et al. Lifetime risk of developing chronic obstructive pulmonary disease: a longitudinal population study. Lancet 2011; 378 : 964-965, 991-996.

11. Roche N, Housset B, Huchon G. Faut-il dépister la BPCO dans la population ? Rev Mal Respir $2008 ; 25$ : 787-90

12. Global Initiative for Chronic Obstructive Lung Disease (GOLD). Revised 2011 at www.goldcopd.org

13. Critères de WILSON (OMS 71). Citation Plan d'action du ministère de la santé français BPCO 2005-2010 p. 27.

14. Edition Française de la série standardisation des explorations fonctionnelles respiratoires du groupe de travail ATS/ERS. Rev Mal Respir 2006 ; 23 : 17S1-175.

15. Bougrida M, Ben Saad H, Bourahli M-K, Bougmiza I, Mehdioui H. Equations de référence spirométriques des Algériens âges de 19 à 73 ans. Rev Mal Respir 2008 ; 25 : 577-90.

16. Ben Saad H, Ben Attia Saafi R, Rouabti S, et al. Quelle définition fautil choisir pour le déficit ventilatoire obstructif ? Rev Mal Respir $2007 ; 24: 323-30$.

Cet article a été publié dans le « Batna Journal of Medical Sciences » BJMS, l'organe officiel de "I'association de la Recherche Pharmaceutique - Batna»

Le contenu de la Revue est ouvert « Open Access » et permet au lecteur de télécharger, d'utiliser le contenu dans un but personnel ou d'enseignement, sans demander l'autorisation de l'éditeur/auteur.

Avantages à publier dans BJMS :

- Open access : une fois publié, votre article est disponible gratuitement au téléchargement

- Soumission gratuite : pas de frais de soumission, contrairement à la plupart des revues "Open Access »

- Possibilité de publier dans 3 langues : français, anglais, arabe

- Qualité de la relecture : des relecteurs/reviewers indépendants géographiquement, respectant

l'anonymat, pour garantir la neutralité et la qualité des manuscrits.

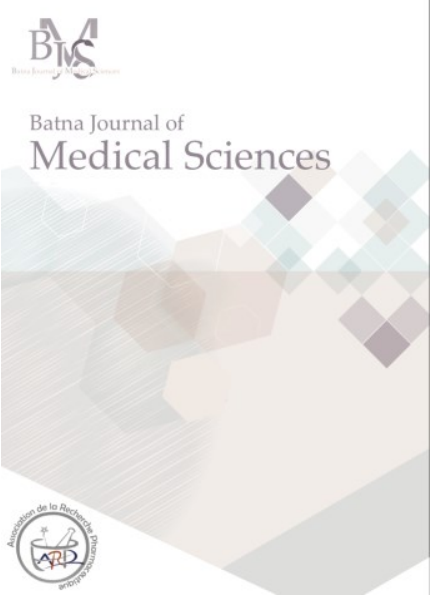




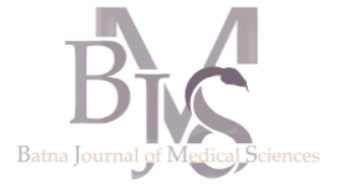

Cardiologue libéral, Cité des

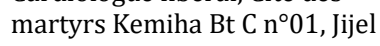
18000 - Algérie

\section{Correspondance à :}

Mohamed MANSOURI

cardimed@hotmail.com

DOI :https://doi.org/10.48087/ BJMSra.2015.2105

\section{Il s'agit d'un article en libre} accès distribué selon les termes de la licence Creative Commons Attribution International License (CC BY 4.0), qui autorise une utilisation, une distribution et une reproduction sans restriction sur tout support ou format, à condition que l'auteur original et la revue soient dûment crédités.

\section{Apports sodés et hypertension artérielle}

\section{Sodium intake and arterial hyertension}

\section{Mohamed Mansouri}

\section{RÉSUMÉ}

Le lien entre le sodium et l'hypertension artérielle est maintenant bien établi mais il demeure discutable dans la population générale. De nombreuses études ont pu démontrer le rôle du sodium dans la régulation normale de la pression artérielle et dans la physiopathologie de l'hypertension artérielle. Toutes les recommandations internationales concernant le traitement non pharmacologique de l'hypertension artérielle proposent de limiter les apports sodés chez les patients hypertendus dans le but d'améliorer le contrôle de l'hypertension artérielle dans la population traitée ou non traitée. De ce fait, une politique de santé efficace passe entre autres par l'adoption de normes nationales sur l'apport alimentaire en sodium de même que par une législation favorisant un étiquetage explicite de la teneur en sodium des aliments préparés.

Mots-clés : apports sodés, hypertension artérielle, sel.

\begin{abstract}
The link between sodium and arterial hypertension is well established; however, it remains questionable whether this is applicable on the general population. Many studies have shown the role of sodium in the normal regulation of blood pressure and in the pathogenesis of arterial hypertension. All the international recommendations dealing with the non-pharmacological treatment of arterial hypertension suggest a limitation of sodium intake in hypertensive patients in order to better control their disease, either pharmacologically treated or not. Therefore, an efficacious health policy must pass through the adoption of national standards concerning diet intake of sodium as well as by regulations favoring the explicit labeling of the amount of salt in each prepared dish.
\end{abstract}

Keywords: sodium intake, arterial hypertension, salt.

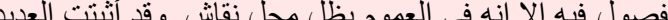

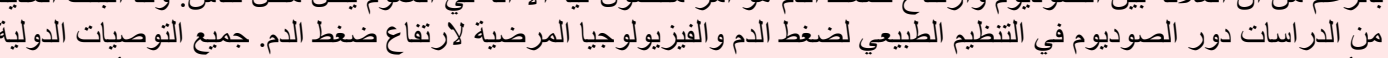

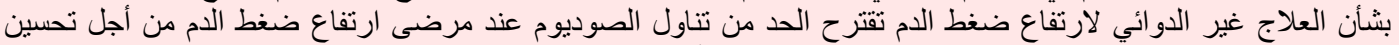

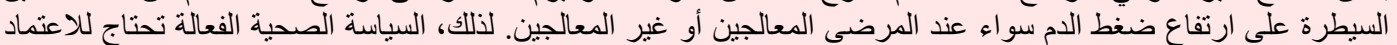

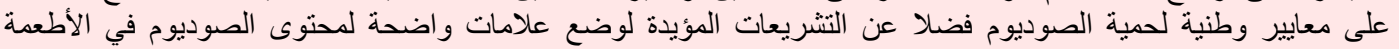

\section{INTRODUCTION}

Le sel de table ou sel alimentaire est presque totalement constitué de chlorure de sodium même s'il contient aussi, en faible proportion, des oligo-éléments comme le fluor ou l'iode. $\mathrm{Au}$ Moyen Âge, il servait à augmenter la durée de conservation des aliments en limitant la multiplication des micro-organismes. Son utilisation s'amplifie au cours du XXe siècle pour devenir largement utilisé par l'industrie agroalimentaire.

S'il semble acquis qu'une limitation des apports en sel réduit la survenue des événements cardiovasculaires dans une population d'hypertendus, le problème est tout autre dans la population générale et différents spécialistes s'affrontent sur la nécessité ou pas d'une réduction de l'apport alimentaire en sel avec des points de vue radicalement opposés. Toutefois, la plupart des agences de santé suivent les recommandations allant dans le sens d'une réduction des apports sodés, les points de vue opposés restant minoritaires.

\section{ETAT DES LIEUX}

La consommation moyenne de sel chez les algériens est estimée entre 9 et $10 \mathrm{~g}$ par jour. Les femmes consommeraient un peu moins que les hommes mais l'apport devient comparable quand il est rapporté à la masse corporelle [1]. 
Chez les hypertendus, les recommandations de l'OMS sur la prise en charge de l'hypertension artérielle essentielle proposent toujours de limiter la consommation en sel $(\mathrm{NaCl})$ à $6 \mathrm{~g} / \mathrm{j}$ (ou $100 \mathrm{mmol} / \mathrm{j}$ ou 2,4 g de sodium/j) [2]; cependant, en Algérie, on consomme 4 grammes rien qu'en mangeant une baguette de pain. Il faut, également, relever que le changement des habitudes alimentaires des Algériens, ces vingt dernières années, a largement favorisé l'apparition de maladies liées à l'hypertension. La restauration rapide, l'abandon de la cuisine traditionnelle basée sur les légumes et le stress sont des facteurs qui sont à l'origine de la propagation de cette maladie. Les Algériens sont aujourd'hui en train de subir les conséquences de l'"amélioration" du niveau de vie et de la surconsommation des glucides, des viandes, des matières grasses et des boissons gazeuses, au même titre que les pays développés [3].

\section{CE QUE DISENT LES RECOMMANDATIONS}

Les recommandations de l'OMS citées plus haut sur la prise en charge de l'HTA essentielle sont rejointes par les recommandations américaines dont les plus récentes sont celles du JNC 8 [4] ou britanniques (NICE 2011) [4][5]. Cette mesure concerne tous les patients hypertendus quel que soit le niveau tensionnel, avec ou sans traitement pharmacologique associé.

Les recommandations sur le traitement médicamenteux du diabète de type 2 soulignent également l'importance des mesures hygiéno-diététiques dans leur globalité et citent l'importance du régime désodé dans le chapitre sur l'hypertension du diabétique.

Les recommandations européennes rappellent que l'apport quotidien recommandé chez les hypertendus se situe aux alentours de $4 \mathrm{~g} / \mathrm{j}$ mais reconnaissent que cet objectif est difficile à atteindre si bien qu'un objectif plus réaliste de moins de $5 \mathrm{~g} / \mathrm{j}$ est proposé. Elles rappellent qu'une alimentation trop salée est une cause d'HTA résistante à rechercher à l'interrogatoire avant toute modification éventuelle de traitement [6].

\section{AVONS NOUS DES RECOMMANDATIONS SOLIDES ?}

\section{Sel et pression artérielle}

Une réduction de l'apport sodé jusqu'à environ $100 \mathrm{mmol} / \mathrm{j}$ se traduit par une baisse de la pression artérielle systolique (PAS) de $6 \mathrm{mmHg}$ et de la pression artérielle diastolique (PAD) de $3 \mathrm{mmHg}$ chez l'hypertendu [7]. Elle peut faciliter le contrôle tensionnel d'un hypertendu et contribuer à la diminution du traitement antihypertenseur. Une réduction des apports sodés à l'échelon d'une population permet de réduire les pressions artérielles de façon dose-dépendante, aussi bien chez l'hypertendu que chez le "pré-hypertendu" [8]. Les réponses interindividuelles sont hétérogènes, tributaires d'influences environnementales, comportementales et génétiques : une revue Cochrane récente sur 167 études conclut, chez l'hypertendu, à une baisse de la PAS de $-5,5 \mathrm{mmHg}$ chez le caucasien, de $-6,4 \mathrm{mmHg}$ chez le sujet noir et de $-10,2 \mathrm{mmHg}$ chez le sujet asiatique [9]. Mais, au final, les pressions artérielles sont un marqueur intermédiaire de risque : qu'en est-il sur la morbimortalité cardiovasculaire?

\section{Apports sodés et événements cardiovasculaires}

Les données restent controversées. Il a été estimé, aux États-
Unis, qu'un programme permettant une réduction de $3 \mathrm{~g} / \mathrm{j}$ de la consommation de sel diminuerait le nombre d'insuffisances coronaires de novo de 60 à 120000 par an, celui des AVC de 32 à 66000 , celui des IDM de 54 à 99000 et que le nombre de décès serait quant à lui diminué de 44 à 92000 , soit un bénéfice estimé comparable à celui attendu d'un programme de sevrage tabagique. Le rapport coût-efficacité semble même plus marqué que celui d'un traitement antihypertenseur [10].

Une méta-analyse suggère une augmentation du risque d'AVC de $6 \%$ pour une augmentation des apports sodés de 50 $\mathrm{mmol} / \mathrm{j}$ tandis que l'impact sur les événements cardiovasculaires est à la limite seulement de la significativité [11]. Le suivi observationnel des patients issus des études randomisées TOHP, chez des adultes "pré-hypertendus" soumis à une réduction des apports sodés de 25 à $35 \%$, montre qu'outre la baisse des pressions artérielles, on assiste à une réduction de la survenue des événements cardiovasculaires de $30 \%$ après ajustement sur les facteurs confondants sur les 10 à 15 années de suivi [12]. C'est dans des populations très consommatrices de sel qu'une relation causale continue indépendante et directe entre apport sodé et événements cardiovasculaires est démontrée.

À l'inverse, une méta-analyse sur 6250 patients qui concerne hypertendus et normotendus, si elle démontre que la réduction sodée s'accompagne d'une baisse de la natriurèse et d'une baisse modeste des PA, ne parvient pas à montrer de baisse significative de la mortalité et de la morbidité cardiovasculaires dans ces deux populations. Les auteurs estiment que, si effet positif il y a, il demeure très limité [13].

Chez des patients diabétiques de type 1, une association entre sel, morbimortalité et insuffisance rénale est trouvée mais pas dans le sens escompté puisque ce sont des apports bas qui sont associés à l'insuffisance rénale et non l'inverse [14]. Dans le diabète de type 2 , une association entre mortalité et natriurèse basse est aussi trouvée ce qui fait dire aux auteurs que si la relation causale n'est pas démontrée, des études d'intervention sont nécessaires [15].

Finalement, le vibrant plaidoyer en faveur d'une réduction des apports sodés dans la population américaine témoigne des enjeux importants de santé publique [16].

Toutefois, lorsque c'est la prise en charge diététique globale qui est prise en compte, une amélioration des événements cardiovasculaires se dessine avec une réduction de risque de décès cardiovasculaires de $35 \%$, d'IDM de $14 \%$ et d'AVC de $19 \%$ chez des patients traités en prévention secondaire [17].

\section{COMMENT FAIRE EN PRATIQUE?}

\section{Les sources alimentaires de sel}

Le sodium est nécessaire au bon fonctionnement de l'organisme et une consommation quotidienne d'au moins $2 \mathrm{~g}$ de sel est indispensable. Le sel naturellement présent dans les aliments ou les boissons représente environ $20 \%$ des apports totaux, le sel ajouté à la cuisson et aux plats représente 10 à $20 \%$ des apports quotidiens (soit 1 à $2 \mathrm{~g} / \mathrm{j}$ ) tandis que $70 \%$ du sel consommé est dû aux aliments manufacturés. L'apport en sel est directement corrélé à la prise énergétique totale.

D'après l'enquête INCA1, six groupes d'aliments les plus contributeurs de sel sont identifiés, qui représentent $70 \%$ des apports en sel en moyenne et $80 \%$ des apports chez les forts consommateurs de sel :

- pain et biscottes (qui apportent $25 \%$ du sel), - charcuteries (13\%), 
- soupes $(10 \%)$,

- fromages ( $9 \%)$,

- plats cuisinés (9\%),

- quiches, pizzas et pâtisseries salées (4 \%).

Deux autres postes importants chez les enfants, par leur fréquence de consommation, sont également pris en compte dans une enquête de l'Anses évaluant la baisse de la quantité de sel entre 2003 et 2011 : les viennoiseries et les céréales [18]. Ainsi, on assiste à une baisse de la quantité de sel dans tous les postes excepté pour les viennoiseries et la charcuterie. Entre 2003 et 2011, on assiste aussi à des modifications des contributions des groupes d'aliments à l'apport sodé (Tableau $1)$.

Tableau 1. Comparaison des 11 premiers groupes d'aliments vecteurs des apports en sel entre INCA 1 et INCA 2 chez les adultes (> 18 ans).

\begin{tabular}{lccc}
\hline Libellé & $\begin{array}{c}\text { Moyenne } \\
\text { des apports } \\
\text { en sel (mg) }\end{array}$ & \multicolumn{2}{c}{$\begin{array}{c}\text { Ordre de contribution } \\
\text { des apports en sel }\end{array}$} \\
& & InCa 1 & InCa 2 \\
\hline $\begin{array}{l}\text { Pain et panification } \\
\text { sèche }\end{array}$ & 1967 & 1 & 1 \\
Charcuterie & & & \\
Condiments et sauces & 652 & 2 & 2 \\
Plats composés & 627 & 5 & 3 \\
Fromages & 522 & 4 & 4 \\
Soupes et bouillons & 440 & 3 & 5 \\
Pizzas,quiches, & 306 & 6 & 7 \\
pâtisseries salées & & & \\
Légumes (hors & 264 & $/$ & 8 \\
pommes de terre) & & & \\
Poissons & 226 & $/$ & 9 \\
$\begin{array}{l}\text { Sandwichs } \\
\text { Pâtisseries etgâteaux }\end{array}$ & 221 & 7 & 10 \\
\hline
\end{tabular}

\section{Des changements nécessaires}

La réduction des apports sodés est une véritable décision politique qui engage la société civile, toute l'industrie agroalimentaire et qui passe par :

- une mobilisation des pouvoirs publics ;

- une communication répétée et intelligente visant à favoriser la diversité alimentaire et une consommation raisonnable des aliments fort contributeurs ;

- une mobilisation sur le terrain au travers, notamment, du médecin généraliste.

L'éducation des patients ne sera possible qu'à condition d'uniformiser l'étiquetage des produits en signalant la quantité de sodium. II est ainsi intéressant de savoir que, lorsque c'est la teneur en sodium $(\mathrm{Na}+)$ qui est mentionnée sur l'étiquette, appliquer un facteur de correction en multipliant par 2,54 permet d'avoir la quantité approchée de sel (ou chlorure de sodium ou $\mathrm{NaCl}$ ). Malheureusement, les diverses enquêtes successives de I'INCA montrent en réalité que la baisse issue de l'évolution des teneurs en sel des aliments les plus contributeurs entre 2003 et 2011 ne se situerait qu'aux alentours de 4 à $10 \%$, soit bien loin des $20 \%$ souhaités. L'Anses préconise donc dans sa conclusion de compléter cet engagement volontaire de réduction du sel par des mesures "le cas échéant réglementaires " donc plus coercitives mais précédées d'une étude d'impact [18].

Une évaluation plus précise des apports en sel est ainsi requise en présence d'une HTA ou d'une insuffisance cardiaque et elle s'intègre dans l'estimation des apports glucido-lipidiques (Tableau 2).
Tableau 2. Sélection de quelques aliments vecteurs de sel dans l'enquête de l'Anses.

\begin{tabular}{|c|c|c|}
\hline Aliment & $\begin{array}{c}\text { Teneur en sel } \\
\text { en } 2003 \\
(\mathrm{~g} / 100 \mathrm{~g})\end{array}$ & $\begin{array}{c}\text { Teneur en sel } \\
\text { en } 2011 \\
(\mathrm{~g} / 100 \mathrm{~g})\end{array}$ \\
\hline Pain, baguette courante & 1,88 & 1,58 \\
\hline $\begin{array}{l}\text { Céréales chocolatées sucrées } \\
\text { enri-chies en vitamines et } \\
\text { minéraux }\end{array}$ & 0,53 & 0,53 \\
\hline $\begin{array}{l}\text { Pétales de maïs natures } \\
\text { enrichies en vitamines et } \\
\text { minéraux }\end{array}$ & 2,27 & 1,72 \\
\hline Croissant ordinaire & 1,35 & 1,37 \\
\hline Croissant au beurre & 1,26 & 1,26 \\
\hline Camembert & 1,53 & 1,54 \\
\hline Emmental & 0,77 & 0,64 \\
\hline Fromage bleu d'Auvergne & 2,96 & 2,84 \\
\hline Fromage de chèvre & $1,51-1,73$ & $1,55-1,69$ \\
\hline Jambon cru & 5,43 & 4,94 \\
\hline Jambon cuit & $1,86-1,93$ & $1,70-1,87$ \\
\hline Saucisson sec & 4,73 & 4,94 \\
\hline Pizza royale & 1,44 & 1,09 \\
\hline $\begin{array}{l}\text { Soupe aux poireaux } \\
\text { préemballée à réchauffer }\end{array}$ & 0,73 & 0,69 \\
\hline Raviolis viande sauce tomate & 1,19 & 0,89 \\
\hline
\end{tabular}

\section{Quelques recommandations}

Les conseils sont personnalisés, adaptés au patient et restent simples comme :

- ne pas resaler avant de goûter, éviter la salière sur la table ;

- réduire drastiquement le sel dans les eaux de cuisson ou dans la poêle ;

- apprécier la quantité journalière de pain (une demibaguette $=100 \mathrm{~g}$, c'est $1,6 \mathrm{~g}$ de sel) ;

- limiter :

$\checkmark$ les biscuits apéritifs (50 g de chips soit un sachet individuel $=1 \mathrm{~g}$ de sel) et les olives ( 4 à 7 olives $=1 \mathrm{~g}$ de sel),

$\checkmark$ la quantité de fromage (les fromages bleus et de chèvre sont les plus salés),

$\checkmark$ la charcuterie (saucisson sec essentiellement),

$\checkmark$ les pizzas ou quiches,

$\checkmark$ les bouillons-cubes, prisés par ceux qui cuisinent et qui perdent le bénéfice de cet effort (ou plaisir)

- faire attention aux soupes, y compris maison (1 petite assiette $=1 \mathrm{~g}$ de sel) ;

- éviter les conserves.

Les eaux minérales plates ou gazeuses contenant plus de 200 $\mathrm{mg} / \mathrm{l}$ de sodium sont à déconseiller. De ce fait, la plupart des eaux minérales plates consommées par les Algériens conviennent généralement aux régimes normaux.

Moins connues, les boissons sucrées, sodas, thés glacés et limonades peuvent contribuer au déséquilibre tensionnel, et pas seulement au travers de l'excès pondéral, et méritent d'être encadrés [19].

Chez l'hypertendu, les récentes recommandations françaises (SFHTA 2013) soulignent que cette réduction sodée s'inscrit si possible dans le cadre d'une éducation thérapeutique. Les mesures peuvent être hiérarchisées et étalées dans le temps avec une réévaluation au cours du suivi. Les Britanniques insistent sur l'importance de l'oral mais aussi de l'écrit (sous la forme de guides ou de conseils pratiques) pour modifier les habitudes alimentaires. Les recommandations européennes disent aux patients de ne pas ajouter de sel aux aliments, d'éviter les aliments trop salés comme les préparations alimentaires industrielles au profit d'aliments cuisinés à partir d'ingrédients naturels riches en potassium [6]. 


\section{COMMENT SURVEILLER ?}

\section{À l'échelon individuel}

L'apport en sel est directement corrélé à la prise énergétique totale. Une réduction des apports sodés peut se traduire par une perte pondérale, rapide les premières semaines car la rétention hydrosodée est réduite, plus lente par la suite, la traduction d'une réduction de l'apport énergétique. Les chiffres de PA s'améliorent indépendamment d'une modification de traitement médicamenteux, notamment chez les gros saleurs. Les patients disent parfois qu'ils ont "dégonflé" avec moins de rétention hydrosodée.

La natriurèse des $24 \mathrm{~h}$ (voire sur $8 \mathrm{~h}$ dans certains travaux) est directement corrélée à la baisse des PA dans des études courtes reprises sous forme d'une méta-analyse : une baisse de $75 \mathrm{mmol} / \mathrm{j}$ est associée à une baisse moyenne des PAS/PAD de $-5 \mathrm{mmHg} /-3 \mathrm{mmHg}$ chez l'hypertendu [20]. Cependant, lors d'un travail longitudinal de 6 ans sur plus de 1000 hypertendus, la corrélation entre PAS (et pas PAD) et natriurèse, bien que de moindre amplitude, est bien retrouvée mais sans démonstration d'un impact sur la morbimortalité [21]. Reste à savoir que si la natriurèse des 24 h est utilisable pour quantifier les apports sodés à l'échelon d'une population, sa variabilité intra-individuelle explique qu'un seul prélèvement ne soit pas le fidèle reflet des apports sodés d'un individu. S'ajoute à cela le fait qu'elle est difficilement réalisable en pratique (un recueil d'urine des $24 \mathrm{~h}$ n'est pas aisé, elle mérite d'être validée par la diurèse souvent disponible, mais aussi par la créatininurie qui ne l'est pas toujours) et interprétable seulement en l'absence de prise de diurétiques ou de bloqueurs du système rénineangiotensine. Lorsque les conditions idéales sont réunies, alors ingérer $6 \mathrm{~g} / \mathrm{j}$ de sel équivaut à une natriurèse aux alentours de $100 \mathrm{mmol}$ par $24 \mathrm{~h}$ et chaque gramme supplémentaire équivaut à rajouter $17 \mathrm{mmol}$ : très instructif par exemple en hospitalisation à l'entrée des patients.

En somme, c'est l'interrogatoire couplé à une analyse diététique plus fine qui, bien souvent, est le seul moyen d'apprécier les prises sodées et la prise d'antihypertenseurs, en plus de toutes les considérations précédentes entravant l'interprétation de la natriurèse. On comprend d'ailleurs toute la difficulté qu'il y a à évaluer une politique de santé publique basée sur la réduction des apports sodés, faute d'indicateurs.

\begin{abstract}
À l'échelon collectif
Une surveillance de l'étiquetage des produits, de la quantité de sel ponctuellement relevée, le suivi de l'application de mesures dans la restauration collective (pas de salière sur la table, réduction de la taille des sachets de sel, alimentation variée et diversifiée) et la pratique d'enquêtes alimentaires sur des populations ciblées, ou pas, complètent le dispositif de surveillance.
\end{abstract}

\section{LE CONCEPT DE SENSIBILITÉ INDIVIDUELLE AU SEL EST-IL VALIDE ?}

La réponse tensionnelle liée aux apports sodés varie d'un patient à l'autre et c'est un phénomène appelé la sensibilité au sel. Cette hétérogénéité de réponse serait tributaire de plusieurs facteurs dont l'âge, le sexe, le niveau tensionnel, le poids, les prises d'alcool, l'activité physique et des facteurs génétiques [21]. Ainsi, il vient d'être montré qu'une activité physique soutenue est inversement corrélée à la sensibilité au sel sur les PA [22]. Une forte sensibilité au sel est associée à une morbimortalité cardiovasculaire accrue [23]. Concrètement, dans la plupart des études, un patient est dit sensible au sel si la différence des PA moyennes mesurée entre un régime salé et un régime peu salé excède $5 \%$, soit une augmentation en moyenne de la PAS/PAD de $+6 /+4$ $\mathrm{mmHg}$. De surcroît, en pratique clinique, il n'est pas rare de constater une amélioration du contrôle tensionnel par la seule réduction des apports sodés, en particulier chez le patient gros saleur, obèse, peu actif et remis en charge, et parfois chez le sujet noir, ce qui nous fait dire que, oui, le concept semble validé.

\section{LES ASSOCIATIONS MÉDICAMENTEUSES ANTIHYPERTENSIVES}

On ne peut qu'extrapoler et se dire (mais pas démontrer) qu'une association de bloqueurs du système rénineangiotensine et de diurétiques thiazidiques qui augmentent la natriurèse, provoque une baisse tensionnelle d'autant plus nette que le patient est sensible au sel. Récemment, un travail mené chez l'hypertendu résistant a comparé une stratégie utilisant en plus d'un sartan, d'un inhibiteur calcique et d'un diurétique thiazidique à faible dose, soit des diurétiques en association (ajout d'antialdostérones et de diurétiques de l'anse), soit des bloqueurs du système rénineangiotensine (IEC et bêtabloquants) : c'est la première stratégie par la déplétion sodée qui semble la plus efficace avec $58 \%$ des patients à l'objectif contre $20 \%$ dans l'autre groupe [24].

\section{CONCLUSION}

Si une réduction des apports sodés semble avoir démontré un bénéfice dans la population hypertendue, un débat persiste sur son impact dans la population générale. Les objectifs fixés par les différentes recommandations sont variables chez l'hypertendu et encore non atteints. Seule une politique de santé publique incitative, notamment auprès de l'industrie agro-alimentaire, couplée à une information renouvelée de la population pourraient laisser présager d'un bénéfice sur la morbimortalité. Dans ce sens, il est indispensable que notre pays adopte une stratégie qui vise à réduire la consommation de sel dans la population pour prévenir l'hypertension artérielle et ses complications cardiovasculaires. Le rôle des autorités est capital ainsi que celui des médias pour sensibiliser les ménages algériens à réduire leur consommation de sel, de sucre et d'aliments favorisant l'obésité, dont les sodas et les aliments gras.

Déclaration d'intérêts : l'auteur ne déclare aucun conflit d'intérêt en rapport avec cet article.

\section{RÉFÉRENCES}

1. Enquête nationale Algérienne SAHA. 2004

2. Witworth JA. 2003 World Health Organization (WHO)/International Society of Hypertension (ISH) statement on management of hypertension. J Hypertens 2003 Nov;21(11):1983-92.

3. Enquête nationale TAHINA-INSP. 2005

4. James PA, Oparill S, Carter BL, et al. 2014 Evidence-Based Guideline for the Management of High Blood Pressure in Adults Report From the Panel Members Appointed to the Eighth Joint National Committee (JNC 8). JAMA 2014;311(5):507-520

5. The clinical management of primary hypertension in adults. Clinical Guideline 127. NICE, August 2011. 
6. Mancia G, De Backer G, Dominiczak A et al. 2007 ESH-ESC Practice Guidelines for the Management of Arterial Hypertension: ESH-ESC Task Force on the Management of Arterial Hypertension. J Hypertens 2007 Sep;25(9):1751-62.

7. Cutler JA, Follmann D, Allender PS. Randomized trials of sodium reduction: an overview. Am J Clin Nutr 1997 Feb;65(2 Suppl):643S$651 S$.

8. Sacks FM, Svetkey LP, Vollmer WM et al. Effects on blood pressure of reduced dietary sodium and the Dietary Approaches to Stop Hypertension (DASH) diet. DASH-Sodium Collaborative Research Group. N Engl J Med 2001 Jan 4;344(1):3-10.

9. Graudal NA, Hubeck-Graudal T, Jurgens G. Effets of low-sodium diet vs high-sodium diet on blood pressure, renin, aldosterone, catecholamines, cholesterol and triglyceride (Cochrane review). Am J Hypertens 2012 Jan;25(1):1-15

10. Bibbins-Domingo K, Chertow GM, Coxson PG et al. Projected Effect of Dietary Salt Reductions on Future Cardiovascular Disease. N Engl J Med 2010 Feb 18;362(7):590-9.

11. Strazzullo P, D’Elia L, Kandala NB et al. Salt intake, stroke and cardiovascular disease: meta-analysis of prospective studies. BMJ 2009 Nov 24;339:b4567

12. Cook NR, Cutler JA, Obarzanek E et al. Long term effects of dietary sodium reduction on cardiovascular disease outcomes: observational follow-up of the trials of hypertension prevention (TOHP). BMJ 2007 Apr 28;334(7599):885-8.

13. Taylor RS, Ashton KE, Moxham T et al. Reduced dietary salt for prevention of cardiovascular disease: a meta-analysis of randomized controlled trials. Am J Hypertens 2011 Aug;24(8):843-53

14. Thomas MC, Moran J, Forsblom C et al. The association between dietary sodium intake, ESRD, and all-cause mortality in patients with type 1 diabetes. Diabetes Care 2011 Apr;34(4):861-6.
15. Ekinci El, Clarke S, Thomas MC et al. Dietary salt intake and mortality in patients with type 2 diabetes. Diabetes Care 2011 Mar;34(3):7039.

16. Appel LJ, Frohlich ED, Hall JE et al. the importance of populationwide sodium reduction as a means to prevent cardiovascular disease and stroke: a call to action from the American Heart Association. Circulation 2011 Mar 15;123(10):1138-43

17. Dehghan M, Mente A, Teo KK et al. Relationship between healthy diet and risk of cardiovascular disease among patients on drug therapies for secondary prevention. A prospective cohort study of 31546 high-risk individuals from 40 countries. Circulation 2012 Dec 4;126(23):2705-12.

18. http://www.anses.fr, last accessed on feb $6^{\text {th }}, 2015$.

19. Stolarz-Skrzypek K. Sugar and salt in the pathogenesis of elevated blood pressure. Hypertens 2011 Apr;57(4):676-8

20. He FJ, MacGregor GA. Effect of longer-term modest salt reduction on blood pressure. Cochrane Database Syst Rev. 2004 ;(3):CD004937.

21. Stolarz-Skrzypek K, Kuznetsova T, Thijs $L$ et al. Fatal and nonfatal outcomes, incidence of hypertension, and blood pressure changes in relation to urinary sodium excretion. JAMA $2011 ; 69(12): 1314-5$.

22. Weinberger MH. Salt sensitivity of blood pressure in humans. Hypertension 1996 Dec;14(12):1461-2

23. Rebholz CM, Gu D, Chen J et al. Physical activity reduces salt sensitivity of blood pressure. Am J Epidemiol 2012 Oct 1;176 Suppl 7:S106-13.

24. Morimoto A, Uzu T, Fujii $T$ et al. Sodium sensitivity and cardiovascular events in patients with essential hypertension. Lancet 1997 Dec 13;350(9093):1734-7.

25. Bobrie G, Frank M, Azizi $M$ et al. Sequential nephron blockade versus sequential renin-angiotensin system blockade in resistant hypertension: a prospective, randomized, open blinded endpoint study. J Hypertens 2012 Aug;30(8):1656-64.

Cet article a été publié dans le "Batna Journal of Medical Sciences » BJMS, l'organe officiel de "I'association de la Recherche Pharmaceutique - Batna »

Le contenu de la Revue est ouvert « Open Access » et permet au lecteur de télécharger, d'utiliser le contenu dans un but personnel ou d'enseignement, sans demander l'autorisation de l'éditeur/auteur.

Avantages à publier dans BJMS :

- Open access : une fois publié, votre article est disponible gratuitement au téléchargement

- Soumission gratuite : pas de frais de soumission, contrairement à la plupart des revues "Open Access »

- Possibilité de publier dans 3 langues : français, anglais, arabe

- Qualité de la relecture : des relecteurs/reviewers indépendants géographiquement, respectant

l'anonymat, pour garantir la neutralité et la qualité des manuscrits. 


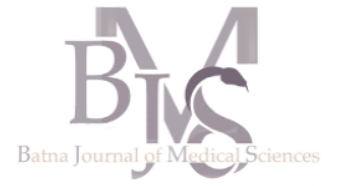

Département de médecine, Université Saad Dahlab, Blida, Algérie

\section{Correspondance à :}

Riad ZERGUINE

\section{z riadh05@hotmail.com}

DOI :https://doi.org/10.48087/ BJMSra.2015.2106

Il s'agit d'un article en libre accès distribué selon les termes de la licence Creative Commons Attribution International License (CC BY 4.0), qui autorise une utilisation, une distribution et une reproduction sans restriction sur tout support ou format, à condition que l'auteur original et la revue soient dûment crédités.

\title{
Peau et soleil
}

\section{Skin and the sun}

\author{
Riad Zerguine
}

\section{RÉSUMÉ}

Le soleil émet une multitude de rayonnements électromagnétiques filtrés par l'atmosphère terrestre. Arrivant à la surface de la planète, ces rayonnements sont indispensables pour l'installation et le développement de la vie. Le spectre solaire au sol comporte les ultraviolets (UV) B (290-320 nm) et les UVA (320-400 nm), la lumière visible et des infrarouges (IR). Les UV activent des molécules, appelées chromophores, contenues dans la peau et qui sont susceptibles de se modifier et ils déclenchent ainsi une cascade de réactions photochimiques ayant des conséquences biologiques et cliniques majeures. Les effets peuvent être soit aigus, soit d'apparition rapide et généralement de courte durée, ou chroniques, d'installation progressive et de longue durée. Les exemples des effets aigus comprennent les coups de soleil et la production de vitamine $\mathrm{D}$, alors que le photovieillissement et le cancer de la peau sont les résultats d'une exposition chronique depuis de nombreuses années. $\mathrm{Si}$ le soleil est indispensable à la vie, son énergie potentiellement destructrice impose à l'homme de savoir l'apprivoiser et de photoprotéger sa peau.

Mots clés : peau, soleil, ultraviolets, photoprotection.

\begin{abstract}
The sun emits a multitude of electromagnetic radiation that is filtered by the earth's atmosphere. When reaching the surface of the planet, this radiation is essential for the installation and the development of life. The solar spectrum on the ground comprises ultraviolet (UV) B (290-320 nm) and UVA (320$400 \mathrm{~nm}$ ), visible light and infrared (IR). UVs activate molecules, called chromophores, contained in the skin and which are likely to change and trigger a cascade of photochemical reactions, with major biological and clinical consequences. Effects can be either acute or rapid onset and usually short, or chronic, with gradual onset and long duration. Examples of acute effects include sunburn and the production of vitamin $\mathrm{D}$, whereas photoaging and skin cancer are the results of chronic exposure for many years. If the sun is essential to life, its potentially destructive energy imposes for man to know tame and photoprotect his skin.
\end{abstract}

Keywords: skin, sun, ultraviolets, photoprotection.

\section{Pour citer l'article :}

Zerguine R. Peau et soleil. Batna J Med Sci 2015;2(1):24-29. https://doi.org/10.48087/ BIMSra.2015.2106

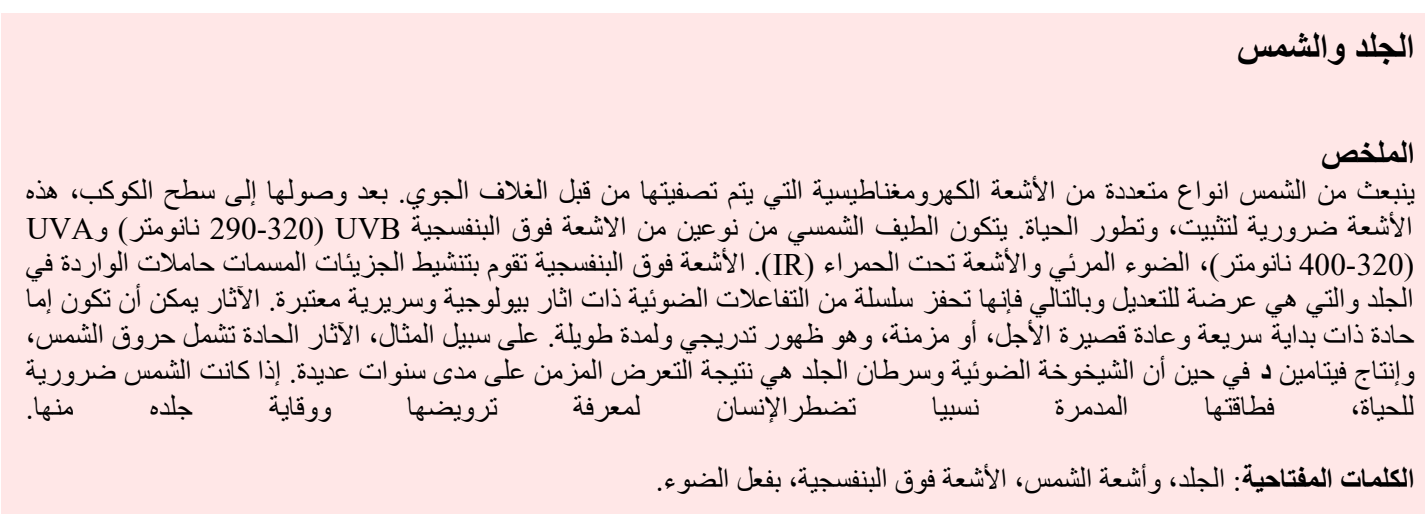

\section{INTRODUCTION}

Les radiations ultraviolettes (UV) sont une composante majeure de notre environnement. Elles sont responsables de nombreux effets moléculaires et biologiques dont certains constituent des signaux de danger pour notre organisme. Au cours de l'évolution, notre organisme a donc développé des mécanismes d'adaptation (réparation, élimination, tolérance, etc.) permettant notre survie [1].
Les effets cliniques observables qui résultent de l'interaction d'un rayonnement optique, en particulier un rayonnement ultraviolet (UV), avec la peau peut être à la fois bénéfique et préjudiciable [2].

\section{BASES PHYSIQUES}

\section{Spectre solaire}

Le soleil, qui est situé à 149 millions de 
kilomètres de la terre, est le siège de réactions thermonucléaires très intenses produisant un immense rayonnement électromagnétique allant des rayons cosmiques aux ondes radioélectriques. La répartition du rayonnement solaire en fonction de l'énergie caractérise le spectre solaire [3].

L'ensemble des rayonnements électromagnétiques est très vaste puisqu'il va des rayons cosmiques jusqu'aux ondes hertziennes (figure1); grandes ondes, ondes moyennes, courtes et ultra-courtes. Ces émissions sont caractérisées par leur longueur d'onde exprimée en nanomètres $\left(1 \mathrm{~nm}=10^{-9} \mathrm{~m}\right)$. Il est à noter que plus la longueur d'onde est grande, plus l'énergie est faible [4].

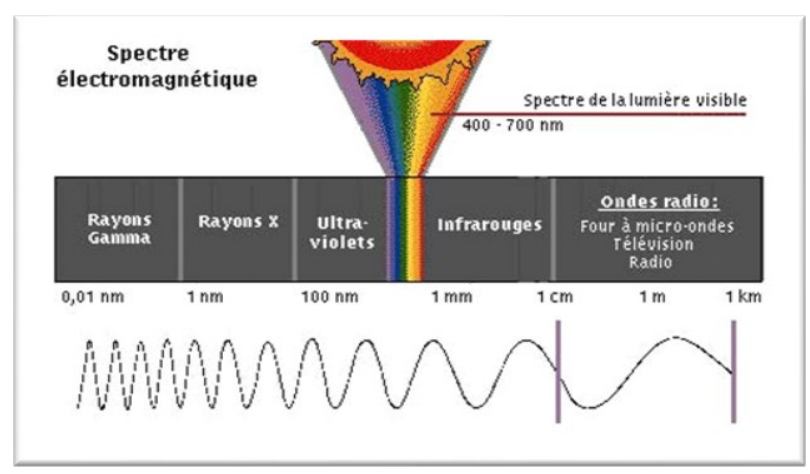

Figure 1: Spectre électromagnétique

\section{Radiations ionisantes}

Comportent les rayons cosmiques, les rayons gamma, les rayons $\mathrm{X}\left(10^{-10}\right.$ à $\left.10^{-9} \mathrm{~m}\right)$

\section{Rayonnement optique : comprend :}

Le rayonnement $U V$, non visible par l'œil humain, divisé en trois zones: UVC de 200 à $280 \mathrm{~nm}$ (arrêtés par le quartz), UVB de 280 à $320 \mathrm{~nm}$ (arrêté par le verre) et UVA de 320 à 400 nm (traversant le verre).

La lumière visible de 400 à $800 \mathrm{~nm}$, décomposée à travers un prisme ou dans un arc-en-ciel : violet, indigo, bleu, vert, jaune, orange et rouge.

Les infrarouges (IR) de 800 à $10000 \mathrm{~nm}$ produisent de la chaleur.

3. Ondes hertziennes : de 1 à $10000 \mathrm{~m}$, n'ont pas d'effets biologiques connus : micro-ondes, ondes radar, télé et radio. Seule une faible partie va atteindre la terre, après avoir été filtrée par l'ozone stratosphérique [3, 4].

\section{Arrivée au sol et pénétration dans la peau}

L'atmosphère terrestre filtre environ le tiers du rayonnement solaire. La couche d'ozone (03) stratosphérique arrête les rayonnements les plus nocifs (rayons cosmiques, rayons gamma, rayons X), les UVC et les UVB les plus courts $(<290$ $\mathrm{nm}$ ) (figure2). La dégradation de la couche d'ozone est donc susceptible d'avoir des effets très néfastes sur toute forme de vie terrestre [5]. D’autres éléments participent à la filtration du rayonnement solaire : les poussières et les fumées qui atténuent plus ou moins la lumière visible, la vapeur d'eau en suspension, les nuages de la basse atmosphère qui absorbent une partie des infrarouges. L'exposition solaire sous un ciel nuageux peut ainsi entraîner un coup de soleil, et ce surtout par une diminution de la sensation de chaleur locale qui entraîne alors une augmentation du temps d'exposition [3].

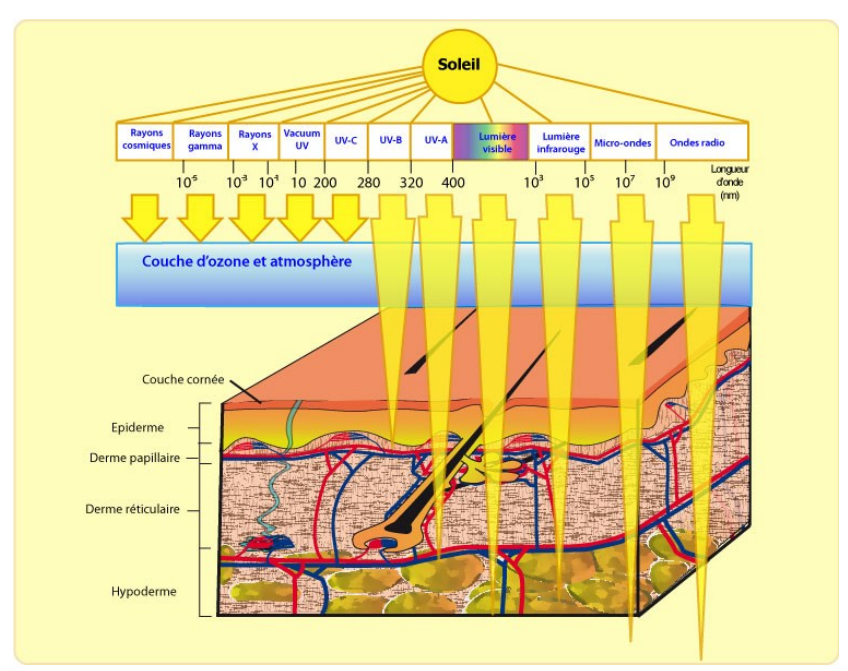

Figure 2: Spectre solaire et pénétration au niveau de la peau

L'ensoleillement reçu par l'individu est la résultante du rayonnement solaire direct, de la lumière du ciel et de la réflexion du sol. La quantité d'UV reçue est variable en fonction de plusieurs paramètres :

- La saison : le taux maximal d'UVB en été est 100 fois supérieur qu'en hiver.

- La latitude : l'ensoleillement étant maximal sous les tropiques où le rayonnement est vertical et où la couche d'ozone est plus faible.

- L'heure du jour : la quantité d'UV est maximale entre $11 \mathrm{~h}$ et $15 \mathrm{~h}$

- L'altitude : la quantité d'UV augmente de $4 \%$ tous les $300 \mathrm{~m}$ $[3,4]$

Arrivées au contact de la peau, certaines radiations lumineuses sont plus ou moins absorbées par des molécules cutanées (kératines, mélanines). La majorité des UVB (70\%) sont arrêtés par la couche cornée ; cependant $20 \%$ atteignent le corps muqueux et $10 \%$ le derme superficiel. La majorité des UVA traversent la couche cornée mais seuls 20 à $30 \%$ atteignent le derme profond. Le visible et l'IR traversent l'épiderme, le derme et parviennent jusqu'à l'hypoderme [4, 6].

\section{LE PHOTOTYPE : CLASSIFICATION DE FITZPATRICK}

Elle permet de classer des sujets en fonction de la couleur de la peau, des cheveux et des yeux, de l'aptitude au bronzage et à prendre des coups de soleil. Il existe six phototypes, plus le phototype est faible, plus le sujet est sensible aux effets du soleil et plus il devra se protéger (tableau 1) [7].

\section{EFFETS BIOLOGIQUES SUR LA PEAU}

Un des plus grands progrès de la photodermatologie au cours de la dernière décennie, est dans la compréhension des 
événements moléculaires au niveau de la peau qui suivent l'exposition aux UV [2].

\begin{tabular}{|c|c|c|}
\hline Phototype & $\begin{array}{l}\text { Couleur de la peau, des } \\
\text { cheveux, et des yeux }\end{array}$ & Réaction au soleil \\
\hline I & $\begin{array}{l}\text { Peau très claire avec des } \\
\text { taches de rousseur } \\
\text { Cheveux blonds ou roux } \\
\text { Yeux clairs }\end{array}$ & $\begin{array}{l}\text { Ne bronze pas } \\
\text { Coups de soleil } \\
\text { systématiques }\end{array}$ \\
\hline II & $\begin{array}{l}\text { Peau très claire } \\
\text { Taches de rousseur au } \\
\text { soleil } \\
\text { Cheveux châtains ou } \\
\text { blonds } \\
\text { Yeux clairs }\end{array}$ & $\begin{array}{l}\text { Bronze difficilement } \\
\text { Coups de soleil } \\
\text { fréquents }\end{array}$ \\
\hline III & $\begin{array}{l}\text { Peau claire } \\
\text { Cheveux châtains ou } \\
\text { blonds }\end{array}$ & $\begin{array}{l}\text { Bronze progressivement } \\
\text { Coups de soleil } \\
\text { occasionnels }\end{array}$ \\
\hline IV & $\begin{array}{l}\text { Peau mate } \\
\text { Cheveux châtains ou bruns } \\
\text { Yeux foncés }\end{array}$ & $\begin{array}{l}\text { Bronze bien } \\
\text { Coups de soleil peu } \\
\text { fréquents }\end{array}$ \\
\hline V & $\begin{array}{l}\text { Peau foncée } \\
\text { Cheveux foncés } \\
\text { Yeux foncés }\end{array}$ & $\begin{array}{l}\text { Bronze facilement } \\
\text { Coups de soleil rares }\end{array}$ \\
\hline VI & $\begin{array}{l}\text { Peau noire } \\
\text { Cheveux foncés } \\
\text { Yeux foncés }\end{array}$ & $\begin{array}{l}\text { Jamais de coups de } \\
\text { soleil }\end{array}$ \\
\hline
\end{tabular}

\section{Production de radicaux libres et d'espèces réactives de l'oxygène}

Les UV sont absorbés par des molécules cibles appelées « chromophores ». C'est cette absorption qui déclenche ensuite les réactions photochimiques [3]. Le chromophore activé établit des liaisons avec des macromolécules environnantes ou surtout transmet l'énergie absorbée à des molécules voisines avec création d'espèces réactives de l'oxygène (ERO) appelées radicaux libres. Ces radicaux libres sont des molécules extrêmement réactives, de puissants agresseurs oxydant de molécules biologiques altérant leurs fonctions biologiques. Les cibles de ces radicaux libres sont l'acide désoxyribonucléique (ADN), les lipides des membranes cellulaires et les acides aminés $[6,8]$.

Ces agressions radicalaires vont s'accumuler, altérant les cellules épidermiques et dermiques. Heureusement, ces cellules disposent de systèmes de défense antiradicalaire [4].

\section{Synthèse de la vitamine $\mathrm{D}$}

La vitamine D3 ou cholécalciférol est une vitamine liposoluble qui est synthétisée dans la peau [3], elle survient suite à la transformation épidermique du 7dehydrocholesterol en cholecalciferol sous l'effet des UVB, ensuite hydrolyse par le foie et le rein pour donner la vitamine D active. L'exposition des seules zones habituellement découvertes 10 a 15 minutes, 2 à 3 fois par semaine l'été, suffit à assurer les besoins en vitamine D [9].

La vitamine D est un antioxydant bien connu dans la peau, avec également un rôle important dans le métabolisme du calcium. Un nombre croissant de preuves montre une réduction de différents types de cancers après une supplémentation en vitamine D [10].
Effets immunologiques des radiations ultraviolettes

La plupart des travaux sur l'immunosuppression induite par les UV concernent les effets des UVB. En effet, ils sont beaucoup plus efficaces en termes d'immunosuppression que les UVA. Les UVB peuvent aboutir à la production de nombreux médiateurs, dont certains auront non seulement une action locale, mais également systémique (cytokines, histamine, prostaglandines) [11].

Les UV modifient le nombre, le phénotype et la fonction des cellules de Langerhans (CL). Après irradiation, les CL sont susceptibles d'entrer en apoptose et/ou de perdre leurs processus dendritiques avec des anomalies de migration vers les ganglions lymphatiques $[1,3]$.

En effet, deux ou trois jours après une exposition aiguë à une forte dose d'UVB, les CL ont disparu complètement de l'épiderme et ont été remplacées par des cellules macrophagiques ayant des propriétés particulières de présentation antigénique leur permettant d'activer préférentiellement des lymphocytes $\mathrm{T}$ à activité suppressive [3]. Les UV sont donc susceptibles d'altérer les défenses immunitaires et la résistance à certains agents infectieux. L'herpès cutané est un exemple d'infection virale favorisée par l'exposition solaire [12].

L'importance de l'immunosuppression induite par les UVB sur la croissance de clones tumoraux a été bien démontrée, en particulier pour le mélanome [1].

\section{CONSÉQUENCES CLINIQUES SUR LA PEAU}

\section{Phénomènes précoces}

Ils sont en général bienfaisants

L'action calorique : est due aux infrarouges qui provoquent une vasodilatation intense se traduisant par un érythème immédiat et une élévation de la température cutanée. Par mécanisme reflexe, la sécrétion sudorale assure la thermorégulation. En cas de surexposition, la saturation des possibilités de thermorégulation conduit au coup de chaleur surtout chez les enfants et les vieillards [9].

Pigmentation Immédiate : elle se produit en réponse à de faibles doses (1 à $5 \mathrm{~J} / \mathrm{cm}^{2}$ ) d'UVA; elle survient après l'exposition et disparaît généralement dans les 10 à 20 minutes [2], elle résulte de la photo-oxydation et de la redistribution de la mélanine préexistante, il n'y a pas de nouvelle synthèse de mélanine $[3,9]$.

L'action antidépressive : a été établie sur des constatations cliniques et des essais thérapeutiques en psychiatrie. Son mécanisme parait être lie à l'interaction lumineuse sur la sécrétion de mélatonine hypophysaire, comme en attestent les dosages effectués chez les sujets atteints du syndrome dépressif saisonnier. Il s'agit en fait d'une stimulation lumineuse oculaire et non d'une véritable action cutanée, par ailleurs les longueurs d'onde efficaces se situent dans le visible et non l'UV [9].

\section{Phénomènes retardés}

Coup de soleil, ou érythème actinique : le coup de soleil est une rougeur plus ou moins intense du tégument, survenant quelques heures après une exposition soit à la lumière naturelle, le soleil, soit à une lumière artificielle, cet érythème 
est essentiellement dû aux UVB [13], et une dose d'UVA 1000 fois supérieure est nécessaire pour déclencher un érythème de même intensité [9], il parait être lié à une agression des cellules épidermiques avec libération de médiateurs de l'inflammation [13].

Pigmentation retardée: elle débute 2 à 3 jours après l'exposition et atteint son maximum au bout de 3 semaines. Il s'agit d'une véritable néosynthèse de mélanine avec stimulation de tous les processus du système pigmentaire. Son spectre d'action se superpose grossièrement à celui de l'érythème [2]. Le rôle de la pigmentation dans la photoprotection naturelle est évident et le bronzage parait donc augmenter celle-ci d'un facteur de 10.

Hyperplasie épidermique: après une inhibition initiale apparait une exacerbation mitotique kératinocytaire conduisant finalement à un épaississement de la couche cornée, ce qui est responsable de l'aggravation de certaines dermatoses comme l'acné [14]; le soleil est un faux amis des acnéiques.

\section{Effets à long terme}

L'action du soleil sur la peau est cumulative. L'apparition des effets chroniques dépend de deux facteurs : la dose totale de photons reçue et la qualité de photoprotection naturelle de l'individu.

Héliodermie : le vieillissement cutané comprend 2 phénomènes distincts : le vieillissement intrinsèque, secondaire au passage du temps qui est chronobiologique, et le photovieillissement, secondaire à l'exposition solaire, appelé l'héliodermie.

Le photovieillissement cutané s'observe au niveau des zones habituellement exposées au soleil, comme la face, le dos des mains, les avant bras et le décolleté. Généralement, les patients à peau claire (phototypes I et II) sont plus touchés [15], Il résulte des effets cumulés et synergiques entre les UVB, les UVA et les IR sur les cellules épidermiques et dermiques [8].

Les taches pigmentées, les rides et les télangiectasies constituent les premières manifestations de l'exposition solaire chronique; la peau devient ensuite épaissie, jaunâtre, plus sèche, les rides se creusent et une pigmentation irrégulière apparaît associant des tâches hyper- et hypopigmentées, des éphélides et des lentigos actiniques (figure3). Enfin, sur la peau chroniquement insolée peuvent apparaître des kératoses actiniques (figure 4), et des carcinomes. Plusieurs aspects cliniques peuvent être individualisés :

- La nuque rhomboïdale souvent observée chez les agriculteurs et les jardiniers (figure 5) ; peau épaissie et marquée par des rides profondes pouvant former des losanges.

- Une peau épaisse et jaunâtre avec des orifices folliculaires dilatés (peau citréine de Milian)

- L'erythrosis interfollicularis colli, siégeant sur les faces latérales du cou où la peau $\mathrm{y}$ a un aspect de poulet déplumé sur un fond couperosique.

- L'élastoïdose à kystes et à comédons de Favre et Racouchot (figure 6), avec sur les régions temporales et nasales des papules jaunâtres, des kystes et des comédons associés parfois à une hypertrichose $[8,15,16]$

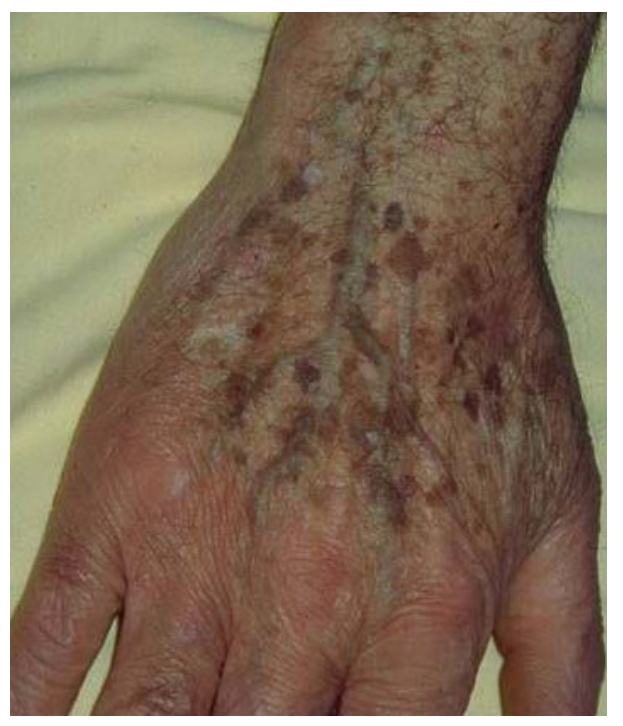

Figure 3 : Lentigos actiniques

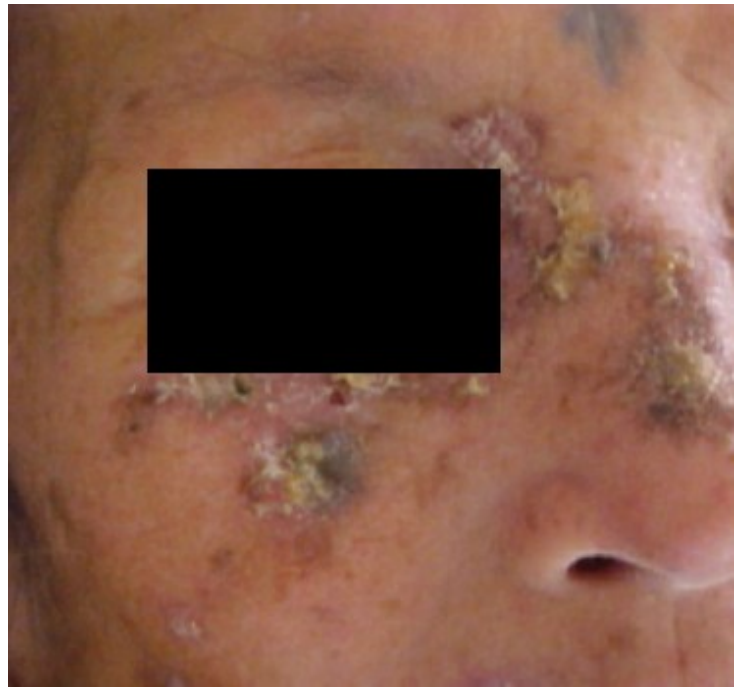

Figure 4 : kératoses actiniques

Photocarcinogenèse : L'effet carcinogène des UV explique la plus grande fréquence des carcinomes cutanés sur les régions exposées à la lumière (visage), chez les sujets à peau claire, travaillant à l'air libre (marins, agriculteurs), vivant dans des régions très insolées. Les expositions solaires brutales et intenses pendant l'enfance et l'adolescence constituent un des principaux facteurs de risque de survenue des mélanomes à l'âge adulte. Les carcinomes spinocellulaires (figure 7), sont corrélés à la quantité totale de radiations reçue alors que certains basocellulaires et les mélanomes sont liés aux expositions intermittentes. [3, 4].

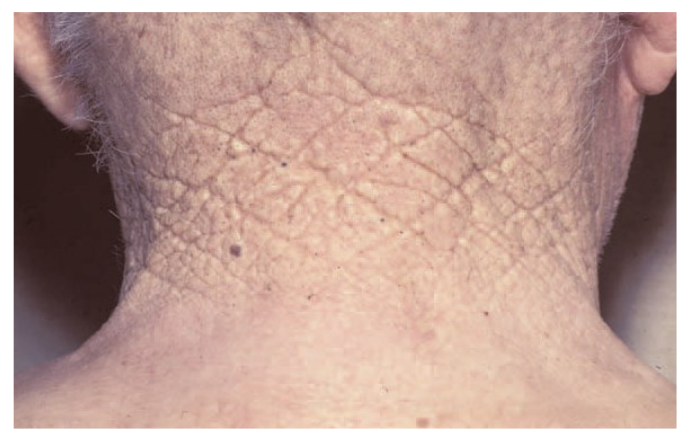

Figure 5 : Nuque rhomboïdale 
L'irradiation UV entraîne de nombreuses perturbations épidermiques dont certaines sont fortement suspectes de participer à la photocarcinogenèse, notamment les altérations de l'ADN, la production de radicaux libres et l'immunosuppression photo-induite. [9].

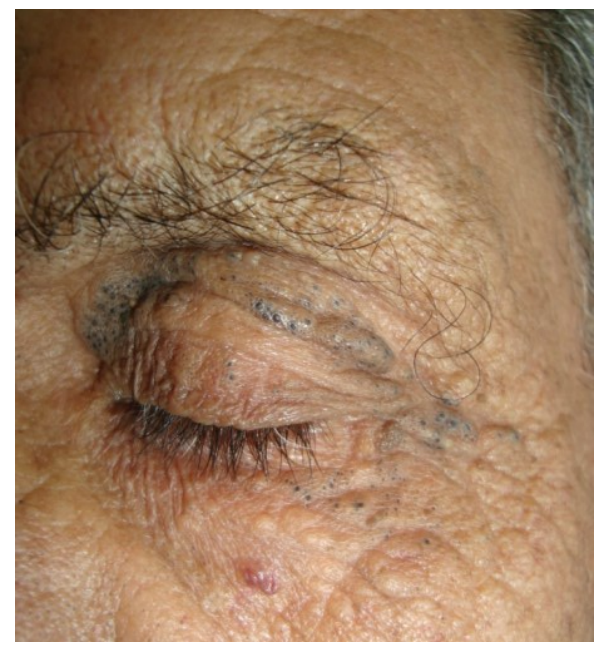

Figure 6 : Elastoïdose à kystes et à comédons de Favre et Racouchot

\section{PREVENTION DES EFFETS SECONDAIRES DU SOLEIL : PHOTOPROTECTION}

La photoprotection regroupe divers moyens naturels et/ou artificiels capables de s'opposer aux dommages cutanés provoqués par les radiations solaires. Les mécanismes physiologiques de la photoprotection naturelle répondent à cet objectif, mais sont insuffisants pour protéger une peau normale mais surexposée ou une peau anormalement photosensible [17].

\section{Photoprotection naturelle:}

- La pilosité: permet en grande partie de prévenir la survenue des effets délétères au niveau du cuir chevelu.

- La couche cornée: qui est photoprotectrice par plusieurs mécanismes (réflexion, diffraction et absorption).

- Les lipides de surface: présents dans le sébum sont capables d'absorber les UVB.

- La mélanine: produite par les mélanocytes et distribuée dans les kératinocytes avoisinants est un des principaux mécanismes de protection, la mélanine absorbe la grande majorité des UV ayant franchi la couche cornée et capte les radicaux libres [18].

- Systèmes de réparation de l'ADN: permettent d'éviter la survenue de mutations photo-induites [17].

- Systèmes antioxydants endogènes (catalase, superoxyde dismutase, glutathion réductase, vitamines $\mathrm{C}$ et $\mathrm{E}$ ) : jouent un rôle très important dans la prévention des effets secondaire du soleil.

\section{Produits de protection solaire (PPS):}

On distingue deux types de PPS :

- Les filtres chimiques: Ce sont des molécules synthétiques qui agissent par absorption sélective des photons en fonction de la longueur d'onde.

- Les poudres minérales : qui réfléchissent et diffractent le rayonnement indépendamment de la longueur d'onde.

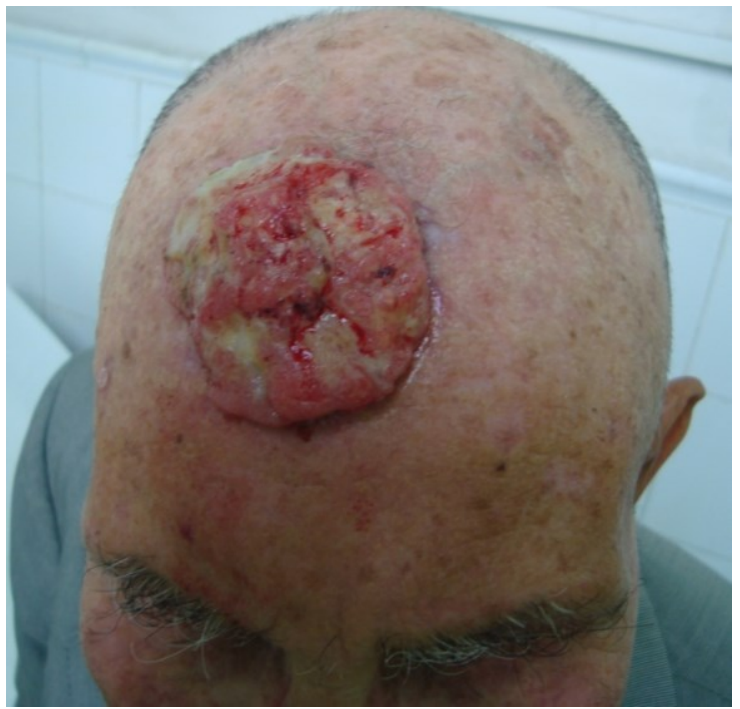

Figure 7 : Carcinome spinocellulaire avec de multiples kératoses et lentigos actiniques

Le critère d'efficacité primaire pour un PPS repose sur son facteur de protection solaire (FPS) mais il doit aussi avoir un spectre large dans l'UVA, l'UVB et l'infrarouge.

Le FPS est mesuré par le rapport entre les mesures de la dose d'UVB nécessaire pour provoquer une rougeur sur une zone non protégée et sur une zone protégée par le PPS.

En clair : Avec un indice FPS 30, il faut une dose 30 fois plus importante d'UVB pour provoquer un coup de soleil $[19,20]$.

Les PPS sont classés en quatre niveaux de protection en fonction du FPS affiché :
- Photoprotection faible
FPS 6 ou 10
- Photoprotection moyenne FPS 15, 20 ou 25
- Photoprotection haute FPS 30 ou 50
- Photoprotection très haute FPS 50+ [17].

\section{Photoprotection vestimentaire}

Un habillement adapté procure un filtre optique contre la pénétration des radiations néfastes. Les chapeaux à large bord protègent les oreilles, le nez, le front et complètent la protection offerte par les cheveux.

Le coefficient de protection des vêtements est très variable selon la texture, la couleur et l'épaisseur [20].

Les tissus les plus protecteurs sont la serge de coton, la soie, le polyester réfléchissant. Les vêtements foncés sont les plus efficaces mais ils absorbent les infrarouges, ce qui les rend inconfortables $[9,17]$.

\section{Conseils simples avant de s'exposer au soleil}

- Pratiquer des expositions d'autant plus progressives que la peau est plus claire.

- Eviter de s'exposer entre 11 et 15 heures, lorsque l'irradiation UVB est maximum

- Eviter la position immobile type « bain de soleil »

- Se méfier de la réflexion sur le sol (eau, sable, neige) et de la fausse protection offerte par le ciel couvert ou le vent.

- Ne pas oublier la protection des yeux (casquette, lunettes). 
- Ne jamais s'exposer après application de produits parfumés ou lors de la prise de certains médicaments potosensibilisants (cyclines, rétinoïdes ...)

- Appliquer régulièrement (toutes les 2 à 3 heures) un photoprotecteur performant dont le but n'est pas d'augmenter le nombre total d'heures d'exposition ni de promouvoir un bronzage intense, mais de permettre une exposition raisonnable au moindre risque.

- Chez l'enfant, la photoprotection doit être rigoureuse car la dose lumineuse reçue est très importante a cette période et c'est un moment crucial pour l'induction des mélanomes, cette protection doit être avant tout vestimentaire, et complétée par les antisolaires.

- Eviter l'exposition solaire directe avant l'âge de 3 ans.

\section{CONCLUSION}

Le spectre ultraviolet (UVB, mais aussi les UVA) est responsable des principaux effets aigus et chroniques après absorption par la peau. Cependant, les UV ne représentent qu'une faible portion du spectre solaire, et il est vraisemblable que les rayonnements visible et infrarouge exercent également des effets délétères. Ces conséquences cliniques sont devenues un motif de consultation très fréquent en dermatologie, néanmoins, tous ses effets délétères ne peuvent être traités. Les prévenir reste le meilleur moyen d'éviter le recours à des techniques de réparation souvent agressives et décevantes.

Déclaration d'intérêts : l'auteur ne déclare aucun conflit d'intérêt en rapport avec cet article.

\section{RÉFÉRENCES}

1. Aubin F. Photo-immunologie. Effets immunologiques des radiations ultraviolettes et implications en dermatologie, Encyclopédie Médico-chirurgicale 2012 : 98-780-A-10

2. Henry W, Herbert H, John L.M. In Photodermatology (Informa) 2007: $76-85$

3. Meunier L. Rayonnement solaire : bases physiques, effets cutanés biologiques et cliniques, Encyclopédie Médico-chirurgicale 2012 : 50-020-B-40
4. Bejar J. Peau, soleil et photosensibilisation médicamenteuse exogène : exemple du kétoprofène, Pharmaceutical sciences. 2012 : dumas-00787853

5. Ballare $\mathrm{CL}$, Caldwell MM, Flint SD et al. Effects of solar ultraviolet radiation on terrestrial ecosystems. Patterns, mechanisms, and interactions with climate change. Photochem Photobiol Sci 2011;10:226-41.

6. Kchevar irene E, Taylor C R, Krutmann J. Fundamentals of cutaneous photobiology and photoimmunology, fitzpatrick's dermatology in general medicine ; seventh edition , 2008; $797-809$

7. Suzuki HS, Hammerschmidt M, Kakizaki P, et al. Phototype comparison between caucasian and asian skin types, Surg Cosmet Dermatol 2011; (3):193-6.

8. Chung J, Cho S, Kang S. Why Does the Skin Age? ; Photoaging 2004; $1-13$

9. Beani J-C. peau et soleil, Dermatologie et infections sexuellement transmissibles, 5ème édition $2009 ; 399$-419

10. Gorham ED, Optimal Vitamin D. Status for Colorectal Cancer Prevention. Am J Prev Med. 2007;32:210-216.

11. Schwarz T. Mechanisms of UV-induced immunosuppression, Keio J Med 54 (4): 165-171, December 2005

12. Perna JJ, Mannix ML, Rooney JF et al. Reactivation of latent herpes simplex virus infection by ultraviolet light: a human model. J Am Acad Dermatol 1987;17:473-8.

13. Manciet J-R. Coup de soleil et prévention, Encyclopédie Médicochirurgicale 2011 : 1-0340

14. Young Antony R, Walker susan L. acute and chronic effects of ultraviolet radiation on the skin, fitzpatrick's dermatology in general medicine; seventh edition, 2008; 809-815

15. Halachmi S, Yaar M, gilchrest BA. Avancées dans le domaine du vieillissement cutané et du photovieillissement. Ann Dermatol Venereol 2005;132:362-7

16. Leccia M-T, Vieillissement cutané photo-induit, Encyclopédie Médico-chirurgicale 2006 : 50-050-B-10

17. Meunier L. Photoprotection (interne et externe), Encyclopédie Médico-chirurgicale 2008 : 98-944-A-10

18. Rai R, Srinivas CR. Photoprotection. Indian J dermatol venereol leprol ; March- April 2007, Vol 73, Issue 2

19. Seite S, Fourtanier AMA. The benefit of daily photoprotection, J Am Acad Dermatol 2008;58:S160-6.

20. Lacours J, Beani J. Photoprotection naturelle, photoprotection externe (topique et vestimentaire). Ann Dermato Venereol 2007 ; $134: 18-24$

Cet article a été publié dans le « Batna Journal of Medical Sciences » BJMS, I'organe officiel de "I'association de la Recherche Pharmaceutique - Batna »

Le contenu de la Revue est ouvert « Open Access » et permet au lecteur de télécharger, d'utiliser le contenu dans un but personnel ou d'enseignement, sans demander l'autorisation de l'éditeur/auteur.

Avantages à publier dans BJMS :

- Open access : une fois publié, votre article est disponible gratuitement au téléchargement

- Soumission gratuite : pas de frais de soumission, contrairement à la plupart des revues « Open Access "

- Possibilité de publier dans 3 langues : français, anglais, arabe

- Qualité de la relecture : des relecteurs/reviewers indépendants géographiquement, respectant l'anonymat, pour garantir la neutralité et la qualité des manuscrits.

Pour plus d'informations, contacter BatnaJMS@gmail.com ou connectez-vous sur le site de la revue : www.batnajms.com 


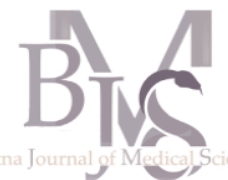

1. Etablissement publique hospitalier Arris, Algérie.

2. Service de physiologie clinique et exploration, $\mathrm{CHU}$ Benflis Touhami Batna 05000, Algérie.

3. Département de pharmacie, Faculté de Médecine, Université Hadj Lakhdar, Batna, Algérie.

4. Département de pharmacie, Faculté de médecine, Université Djilali Liabes, Sidi Bel-Abbés, 22000 Algérie.

5. Laboratoire de pharmacologie, CHU Sidi-Bel-Abbés, Algérie.

\section{Correspondance à :}

Hocine GACEM

ghocine14@gmail.com

DOI:https://doi.org/10.48087/ BJMSoa.2015.2107

Il s'agit d'un article en libre accès distribué selon les termes de la licence Creative Commons Attribution International License (CC BY 4.0), qui autorise une utilisation, une distribution et une reproduction sans restriction sur tout support ou format, à condition que l'auteur original et la revue soient dûment crédités.

\section{Pour citer l'article :}

Aggabi A, Gacem H, Bahri $\mathrm{H}$, et al. L'automédication par les antibiotiques étude auprès de cinq officines pharmaceutiques de cinq villes algériennes. Batna J Med Sci 2015;2(1):30-35 https://doi.org/10.48087/ B]MSoa.2015.2107

\title{
L'automédication par les antibiotiques : étude auprès de cinq officines pharmaceutiques de cinq villes algériennes.
}

\author{
Antibiotics in self-medication: study of five pharmacies from five \\ Algerian cities.
}

\author{
Amina Aggabi' ${ }^{1}$, Hocine Gacem ${ }^{2,3}$, Hafid Bahri ${ }^{4,5}$, Boualem Talha ${ }^{4}$, Abderrahmane \\ Boulerial $^{4}$, Amal Gacem4, Yasmine Koubaa ${ }^{4}$, Amina Yahiaoui ${ }^{4}$, Amel Ahmane 2,3
}

\section{RÉSUMÉ}

Introduction : L'automédication est un phénomène universel menaçant la santé publique surtout en ce qui concerne les antibiotiques car c'est l'un des facteurs favorisant l'apparition de résistance. L'objectif de l'étude était de pré-estimer la prévalence de l'automédication par les antibiotiques (ATBs) et d'en rechercher les motivations poussant à cette pratique ainsi que les ATBs les plus demandés dans cinq villes Algériennes. Matériel et méthode : C'est une étude multicentrique, descriptive, exhaustive et observationnelle de la pratique de l'automédication par les ATBs au niveau de cinq officines pharmaceutiques réparties sur cinq villes algériennes (Batna, M'sila, Mascara, Tiaret et Sidi-Bel-Abbès) durant une période de 15 jours à raison de 3 heures par jour (de $10 \mathrm{~h} 00$ à $13 \mathrm{~h} 00$ ) par le biais d'un autoquestionnaire anonyme. Résultats: Le sex-ratio moyen était de 1,92 ce qui représente une prédominance masculine avec un âge compris pour la plupart entre 30 et 44 ans. L'antibiotique demandé était le plus souvent pour le demandeur lui-même, poussé par leur expérience antérieure avec des pourcentages allant de $51,4 \%$ à $85,7 \%$ pour les cinq villes. La famille des bétalactamines constitue la principale classe d'antibiotiques demandée. En ce qui concerne les motivations de l'automédication, à Batna et Tiaret le motif principal était la banalisation de la maladie, alors qu'à Mascara s'était surtout pour des raisons économiques. A Sidi-Bel-Abbès d'autres motifs étaient responsables. Enfin, la ville de M'sila a fait l'exception en donnant les différents motifs à parts égales ( $25 \%$ chacune).

Mots clés : automédication, antibiotiques, officines pharmaceutiques, Algérie.

\begin{abstract}
Introduction: Self-medication is a universal phenomenon threatening public health especially regarding antibiotics because it is one of the factors promoting the development of resistance. The aim of the study was to pre-estimate the prevalence of selfmedication by ATBs and seeking motives pushing this practice as well as the most requested ATBs in five Algerian cities. Material and method: This is a multicenter, descriptive, observational and comprehensive practice study of self-medication by antibiotics (ATBs) at five pharmacies on five Algerian cities (Batna, Msila, Mascara, Tiaret and Sidi-Bel Abbès) during a period of 15 days, 3 hours per day (from 10.00 to 13.00 ) via an anonymous selfadministered questionnaire. Results: The average sex ratio was 1,92 with ages ranging mostly between 30 and 44 years. The antibiotic most often asked was for the applicant itself, pushed by their previous experience with percentages ranging from $51.4 \%$ to $85.7 \%$ for the five cities. The beta-lactam was the primary class of antibiotics requested. As for the motives of self-medication, in Batna and Tiaret the main reason was the trivialization of the disease, then that Mascara had largely economic reasons. In SidiBel-Abbes, other reasons were responsible. Finally, the city of M'sila made exception by giving different equal grounds (25\% each).
\end{abstract}

Keywords: self-medication, antibiotics, pharmacies, Algeria.

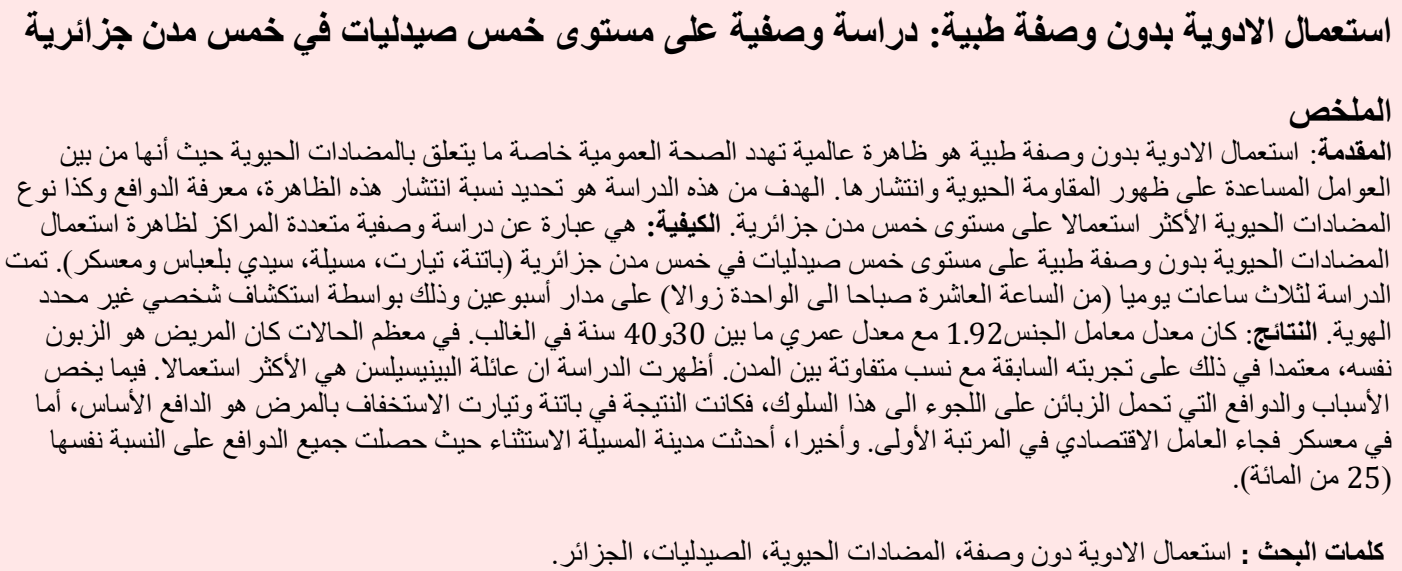




\section{INTRODUCTION}

Depuis la nuit des temps, l'homme a toujours cherché instinctivement à soigner des maux affectant sa santé, et ceci sans forcément avoir recours à un homme de science. C'est ce qu'on appelle "l'autonomie » [1]. Lecomte considère que dans le sens le plus large "l'automédication consiste à faire, devant la perception d'un trouble de la santé, un autodiagnostic et à se traiter sans avis médical » mais que dans un sens plus restreint, c'est « l'acquisition d'un produit sans ordonnance, que l'on nomme automédication » [1].

Selon la définition recommandée par l'OMS, l'automédication est considérée comme l'utilisation des médicaments pour traiter des symptômes ou désordres de la santé autodiagnostiqués [2] ou l'utilisation intermittente ou continue des médicaments prescrits pour des maladies ou symptômes chroniques ou récurrents [3-11]

L'automédication constitue un problème sérieux de santé publique où une prévalence de 10 à $87 \%$ était estimée À travers le monde [12] d'où provient le caractère universel de cette pratique [13-15] qui est rencontrée aussi bien dans les pays en voie de développement que les pays développés [5, 12, 15-19].

L'automédication touche plusieurs classes médicamenteuses, parmi elles les antibiotiques qui ont profondément modifié au début du siècle dernier l'évolution de la thérapeutique antiinfectieuse par la guérison d'infections graves constituant ainsi un bien précieux $[19,20]$. Mais l'apparition de bactéries résistantes aux antibiotiques et leur diffusion dans la population est l'un des phénomènes infectieux majeurs de ces dernières années où l'utilisation intempestive et irrationnelle et la pratique de l'automédication favorise ce phénomène ce qui constitue un problème de santé publique universel $[3,4$, $7,9,14,18-29]$.

Une étude faite en Espagne, incluant 19 régions européennes a rapporté un pourcentage d'automédication par les antibiotiques variant entre 0,1 et $21 \%$ [26]. D'autres prévalences ont été rapportées en Europe : $3 \%$ en Europe du Nord, 6\% en Europe Centrale et 19\% en Europe du Sud, alors que dans les Pays Asiatiques, elle varie de $4 \%$ à 75\% [30].

En ce qui concerne les pays arabes, l'exemple de l'étude menée en 2007 par Yousef AM et al. en Jordanie a mentionné que « à part les tranquillisants, le peuple peut acheter n'importe quel médicament sans prescription médicale » [31].

En effet, nous avons ciblé, par cette étude, l'automédication par les antibiotiques (ATBs) auprès de cinq officines privées réparties sur cinq villes algériennes différentes (Batna, Mascara, M'sila, Sidi-Bel-Abbès et Tiaret). L'objectif de l'étude était de pré-estimer la prévalence de l'automédication par les ATBs et d'en rechercher les motivations poussant à cette pratique ainsi que les ATBs les plus demandés dans cinq villes Algériennes.

\section{MATÉRIELS ET MÉTHODES}

Il s'agit d'une étude multicentrique, descriptive et observationnelle de la pratique de l'automédication par les antibiotiques au niveau de cinq officines pharmaceutiques réparties sur cinq villes algériennes (Batna, M'sila, Mascara, Tiaret et Sidi-Bel-Abbès) durant une période de 15 jours du 22.03.2014 au 05.04.2014, À raison de 3 heures par jour (de $10 \mathrm{~h} 00$ à 13h00) durant la partie de forte activité où les officines reçoivent le plus grand nombre de clients.

L'étude est exhaustive; on a inclus ceux qui viennent demander au moins un ATB sans prescription médicale.
Étaient par contre exclus de l'étude, toute personne ayant refusé de participer, ceux ayant présenté des ordonnances ainsi que les pratiquants de l'automédication par les autres classes médicamenteuses autres que les ATBs. La collection des données a été faite par le biais d'un auto-questionnaire anonyme bilingue (arabe, français), formulé selon les objectifs de notre étude et pré-testé, remplis par les malades ou leurs tuteurs (enfants dont l'âge est inférieur à 18 ans).

Le questionnaire comprend 03 sections, la première concerne les caractéristiques sociodémographiques du demandeur incluant l'âge, le sexe, le niveau d'instruction et la situation professionnelle. La deuxième relative aux ATBs demandés et la troisième section quant à elle concerne les motivations poussant à cette pratique. L'analyse des résultats était réalisée par le logiciel Epi Info 3.5.4 et Excel 2010.

\section{RÉSULTATS}

À l'issue des deux semaines, un total de 159 participants a été inclus dans l'étude. Après exclusion de 13 fiches incomplètes (SBL : 02, Tiaret : 06, Mascara : 03, M'Sila : 02, Batna : 00), le nombre final de répondants inclus a été de 146

Tableau 1. Caractéristiques sociodémographiques de la population.

\begin{tabular}{|c|c|c|c|c|c|}
\hline & $\begin{array}{l}\text { Batna } \\
(\mathrm{N}=7)\end{array}$ & $\begin{array}{l}\text { Mascara } \\
(\mathrm{N}=21)\end{array}$ & $\begin{array}{l}\text { M'sila } \\
(\mathrm{N}=8)\end{array}$ & $\begin{array}{l}\text { Tiaret } \\
(\mathrm{N}=73)\end{array}$ & $\begin{array}{l}\text { SBA } \\
(\mathrm{N}=37)\end{array}$ \\
\hline & $\mathrm{N}(\%):$ & $\mathrm{N}(\%):$ & $\mathrm{N}(\%)$ : & $\mathrm{N}(\%)$ : & $\mathrm{N}(\%):$ \\
\hline \multicolumn{6}{|l|}{ Sexe : } \\
\hline *Homme & $\begin{array}{l}03 \\
(42.9 \%)\end{array}$ & $\begin{array}{l}16 \\
(76.2 \%)\end{array}$ & $\begin{array}{l}04 \\
(50 \%)\end{array}$ & $\begin{array}{l}51 \\
(69.9 \%)\end{array}$ & $\begin{array}{l}26 \\
(70.3 \%)\end{array}$ \\
\hline$*_{\text {femme }}$ & $\begin{array}{l}04 \\
(57.1 \%)\end{array}$ & $\begin{array}{l}05 \\
(23.8 \%)\end{array}$ & $\begin{array}{l}04 \\
(50 \%)\end{array}$ & $\begin{array}{l}22 \\
(30.1 \%)\end{array}$ & $\begin{array}{l}11 \\
(29.7 \%)\end{array}$ \\
\hline Sex-ratios: & 0.75 & 3.2 & 1 & 2.32 & 2.36 \\
\hline \multicolumn{6}{|l|}{ Age: } \\
\hline$*^{*}<15$ ans & 00 & 00 & 00 & $\begin{array}{l}01 \\
(1.36 \%)\end{array}$ & $\begin{array}{l}01 \\
(2.70 \%)\end{array}$ \\
\hline *15-29ans & 00 & $\begin{array}{l}03 \\
(14.28 \%)\end{array}$ & $\begin{array}{l}03 \\
(37.5 \%)\end{array}$ & $\begin{array}{l}12 \\
(16.43 \%)\end{array}$ & $\begin{array}{l}09 \\
(24.32 \%)\end{array}$ \\
\hline * 30-44ans & $\begin{array}{l}06 \\
(85.7 \%)\end{array}$ & $\begin{array}{l}09 \\
(42.85 \%)\end{array}$ & $\begin{array}{l}03 \\
(37.5 \%)\end{array}$ & $\begin{array}{l}32 \\
(43.83 \%)\end{array}$ & $\begin{array}{l}12 \\
(32.43 \%)\end{array}$ \\
\hline *45-59ans & $\begin{array}{l}01 \\
(14.3 \%)\end{array}$ & $\begin{array}{l}06 \\
(28.57 \%)\end{array}$ & $\begin{array}{l}01 \\
(12.5 \%)\end{array}$ & $\begin{array}{l}20 \\
(27.39 \%)\end{array}$ & $\begin{array}{l}09 \\
(24.32 \%)\end{array}$ \\
\hline *60-74ans & 00 & $\begin{array}{l}02 \\
(9.52 \%)\end{array}$ & $\begin{array}{l}01 \\
(12.5 \%)\end{array}$ & $\begin{array}{l}07 \\
(9.58 \%)\end{array}$ & $\begin{array}{l}04 \\
(10.81 \%)\end{array}$ \\
\hline *75-89ans & 00 & $\begin{array}{l}01 \\
(4.76 \%)\end{array}$ & 00 & $\begin{array}{l}01 \\
(1.36 \%)\end{array}$ & $\begin{array}{l}02 \\
(5.40 \%)\end{array}$ \\
\hline$*>90$ ans & 00 & 00 & 00 & 00 & 00 \\
\hline \multicolumn{6}{|l|}{$\begin{array}{l}\text { Niveau } \\
\text { d'instruction : }\end{array}$} \\
\hline *analphabète & 00 & $\begin{array}{l}07 \\
(33.3 \%)\end{array}$ & $\begin{array}{l}01 \\
(12.5 \%)\end{array}$ & 03 (4.1\%) & $\begin{array}{l}04 \\
(10.8 \%)\end{array}$ \\
\hline *primaire & 00 & 00 & 00 & 05 (6.5\%) & $\begin{array}{l}03 \\
(08.1 \%)\end{array}$ \\
\hline *moyen & $\begin{array}{l}01 \\
(14.3 \%)\end{array}$ & $\begin{array}{l}06 \\
(28.6 \%)\end{array}$ & 00 & $\begin{array}{l}16 \\
(21.9 \%)\end{array}$ & $\begin{array}{l}09 \\
(24.3 \%)\end{array}$ \\
\hline *secondaire & $\begin{array}{l}03 \\
(\mathbf{4 2 . 9 \% )}\end{array}$ & $\begin{array}{l}02 \\
(09.5 \%)\end{array}$ & $\begin{array}{l}03 \\
(37.5 \%)\end{array}$ & $\begin{array}{l}26 \\
(35.6 \%)\end{array}$ & $\begin{array}{l}15 \\
(\mathbf{4 0 . 5 \% )}\end{array}$ \\
\hline *universitaire & $\begin{array}{l}03 \\
(\mathbf{4 2 . 9 \% )}\end{array}$ & $\begin{array}{l}06 \\
(28.6 \%)\end{array}$ & $\begin{array}{l}04 \\
(\mathbf{5 0} \%)\end{array}$ & $\begin{array}{l}23 \\
(31.5 \%)\end{array}$ & $\begin{array}{l}06 \\
(16.2 \%)\end{array}$ \\
\hline \multicolumn{6}{|l|}{$\begin{array}{l}\text { Situation } \\
\text { professionnelle : }\end{array}$} \\
\hline *retraité & 00 & $\begin{array}{l}03 \\
(14.3 \%)\end{array}$ & $\begin{array}{l}01 \\
(12.5 \%)\end{array}$ & 08 (11\%) & $\begin{array}{l}07 \\
(18.9 \%)\end{array}$ \\
\hline *étudiant & 00 & $01(4.8 \%)$ & $\begin{array}{l}02 \\
(25 \%)\end{array}$ & 05 (6.8\%) & $\begin{array}{l}06 \\
(16.2 \%)\end{array}$ \\
\hline $\begin{array}{l}\text { *profession } \\
\text { libérale }\end{array}$ & $\begin{array}{l}03 \\
(\mathbf{4 2 . 9 \% )}\end{array}$ & 02 (9.5\%) & $\begin{array}{l}01 \\
(12.5 \%)\end{array}$ & $\begin{array}{l}13 \\
(17.8 \%)\end{array}$ & $10(27 \%)$ \\
\hline $\begin{array}{l}{ }^{*} \text { fonction } \\
\text { publique }\end{array}$ & $\begin{array}{l}02 \\
(28.6 \%)\end{array}$ & $\begin{array}{l}07 \\
(\mathbf{3 3 . 3 \% )}\end{array}$ & $\begin{array}{l}03 \\
(37.5 \%)\end{array}$ & $\begin{array}{l}31 \\
(\mathbf{4 2 . 5 \% )}\end{array}$ & $\begin{array}{l}09 \\
(24.3 \%)\end{array}$ \\
\hline *chômeur & 00 & $\begin{array}{l}06 \\
(28.6 \%)\end{array}$ & $\begin{array}{l}01 \\
(12.5 \%)\end{array}$ & $\begin{array}{l}14 \\
(19.2 \%)\end{array}$ & $\begin{array}{l}04 \\
(10.8 \%)\end{array}$ \\
\hline *autres & $\begin{array}{l}02 \\
(28.6 \%)\end{array}$ & $02(9.5 \%)$ & 00 & $\begin{array}{l}02 \\
(02.7 \%)\end{array}$ & $01(2.7 \%)$ \\
\hline
\end{tabular}


Tableau 2. Destination des antibiotiques achetés sans ordonnance.

\begin{tabular}{llllll}
\hline & $\begin{array}{l}\text { Batna } \\
(\mathrm{N}=7)\end{array}$ & $\begin{array}{l}\text { Mascara } \\
(\mathrm{N}=\mathbf{2 1})\end{array}$ & $\begin{array}{l}\text { M'sila } \\
(\mathrm{N}=\mathbf{8})\end{array}$ & $\begin{array}{l}\text { Tiaret } \\
(\mathrm{N}=\mathbf{7 3})\end{array}$ & $\begin{array}{l}\text { Sidi-Bel- } \\
\text { Abbès } \\
(\mathrm{N}=37)\end{array}$ \\
\hline Vous & $\mathbf{0 3 ( 4 2 . 9 \% )}$ & $\mathbf{0 9 ( 4 2 . 9 \% )}$ & $06(\mathbf{7 5 \%})$ & $30(\mathbf{4 1 . 1 \% )}$ & $13(\mathbf{3 5 . 1} \%)$ \\
Votre conjoint & $01(\mathbf{1 4 . 3 \% )}$ & $01(4.8 \%)$ & 00 & $10(13.7 \%)$ & $03(8.1 \%)$ \\
Votre enfant & $03(\mathbf{4 2 . 9 \% )}$ & $07(33.3 \%)$ & 00 & $27(37 \%)$ & $12(32.4 \%)$ \\
Autres & 00 & $04(19 \%)$ & $02(25 \%)$ & $06(8.2 \%)$ & $09(24.3 \%)$ \\
\hline
\end{tabular}

répartis sur les cinq villes comme suit : 7 à Batna, 8 à M'sila, 21 à Mascara, 73 à Tiaret et 37 à Sidi-Bel-Abbès en donnant des prévalences respectives de l'automédication par les ATBs: $7,6 \% ; 12,5 \% ; 14,4 \% ; 12,6 \%$ et $15,9 \%$ et une prévalence moyenne de $12,6 \%$. Les principaux résultats sont présentés dans les tableaux 1, 2, 3, 4, 5 et la figure1.

Tableau 3. Motivations de l'automédication par les antibiotiques.

\begin{tabular}{lccccc}
\hline & $\begin{array}{c}\text { Batna } \\
(\mathrm{N}=7)\end{array}$ & $\begin{array}{c}\text { Mascara } \\
(\mathrm{N}=21)\end{array}$ & $\begin{array}{c}\mathrm{M}^{\prime} \mathrm{sila} \\
(\mathrm{N}=8)\end{array}$ & $\begin{array}{c}\text { Tiaret } \\
(\mathrm{N}=73)\end{array}$ & $\begin{array}{c}\text { Sidi-Bel- } \\
\text { Abbès } \\
(\mathrm{N}=37)\end{array}$ \\
\hline $\begin{array}{l}\text { Banalisation } \\
\text { de la maladie }\end{array}$ & $04(57.1 \%)$ & $05(23.8 \%)$ & $02(25 \%)$ & $29(39.7 \%)$ & $13(35.1 \%)$ \\
$\begin{array}{l}\text { Manque du } \\
\text { temps }\end{array}$ & $01(14.3 \%)$ & $04(19 \%)$ & $02(25 \%)$ & $19(26 \%)$ & $07(18.9 \%)$ \\
$\begin{array}{l}\text { Motivation } \\
\text { économique }\end{array}$ & $01(14.3 \%)$ & $08(38.1 \%)$ & $02(25 \%)$ & $16(21.9 \%)$ & $01(2.7 \%)$ \\
\begin{tabular}{l} 
Autres \\
\hline
\end{tabular} & $01(14.3 \%)$ & $08(38.1 \%)$ & $02(25 \%)$ & $19(26 \%)$ & $16(\mathbf{4 3 . 2 \% )}$ \\
\hline
\end{tabular}

\section{DISCUSSION}

Ce travail avait comme objectif de porter un regard sur le niveau de l'automédication par les antibiotiques dans le milieu algérien. C'est une étude multicentrique, descriptive des caractéristiques sociodémographiques et les motivations des personnes qui s'adonnent à cette pratique.

La durée de l'étude et le temps de présence au niveau des officines étaiett relativement courts, étalés sur deux semaines au niveau de cinq villes algériennes: Batna, M'sila, Mascara, Tiaret et Sidi-Bel-Abbès a raison de 03 heures par jour, ce qui constitue une limite à cette étude. De ce fait, les informations récoltées ne peuvent être considérées comme des valeurs définitives réelles; néanmoins, elles permettent d'avoir une idée sur l'ampleur du phénomène.

Les prévalences obtenues sont un peu homogènes (entre $12,5 \%$ et $15,9 \%$ ) à l'exception de celle de Batna $(7,6 \%)$ qui est un peu faible. La prévalence moyenne retrouvée $(12,6 \%)$ est proche de celle trouvée à Jos; le nord central du Nigeria $(11,2 \%)$ [32] ainsi qu'en Malaisie, à Hong Kong, en Europe et en Indonésie, où elle se situe entre $4,8 \%$ et $9 \%$ [33].

Tableau 4. Bases du choix de l'ATB.

\begin{tabular}{llllll}
\hline & $\begin{array}{l}\text { Batna } \\
(\mathrm{N}=7)\end{array}$ & $\begin{array}{l}\text { Mascara } \\
(\mathrm{N}=21)\end{array}$ & $\begin{array}{l}\text { M'sila } \\
(\mathrm{N}=8)\end{array}$ & $\begin{array}{l}\text { Tiaret } \\
(\mathrm{N}=73)\end{array}$ & $\begin{array}{l}\text { SBA } \\
(\mathrm{N}=37)\end{array}$ \\
\hline $\begin{array}{l}\text { Expérience } \\
\text { antérieure }\end{array}$ & $06(\mathbf{8 5 . 7 \% )}$ & $12(57.1 \%)$ & $06(\mathbf{7 5 \% )}$ & $39(\mathbf{5 3 . 4 \% )}$ & $19(\mathbf{5 1 . 4 \% )}$ \\
$\begin{array}{l}\text { Conseil d'un } \\
\text { professionnel de } \\
\text { la santé }\end{array}$ & $01(14.3 \%)$ & $07(33.3 \%)$ & $02(25 \%)$ & $26(35.6 \%)$ & $17(45.9 \%)$ \\
$\begin{array}{l}\text { Conseil d'un ami } \\
\text { Autres }\end{array}$ & 00 & $01(4.8 \%)$ & 00 & $05(6.8 \%)$ & $01(2.7 \%)$ \\
\hline
\end{tabular}

Les Palestiniens s'adonnent à l'automédication par les ATBs à un pourcentage de 19\% [17] alors qu'en Jordanie, l'Emirat, le Liban, l'Iraq et le Yémen il y a un saut de la prévalence : entre $40,7 \%$ et $78 \%$ [33] ainsi qu'en Albanie $(78,1 \%$ ) [34].
À Batna, ce sont les femmes qui s'automédiquent plus que les hommes $(57,1 \%$ de femmes contre $42,9 \%$ d'hommes), c'est la même chose retrouvée au Kuwait (68,7\% de femmes contre $31,3 \%$ pour les hommes) [33] ainsi qu'au Yémen, l'Arabie Saoudite et l'Ouzbékistan où les femmes représentent 59,8\%, $84 \%$ et $53,5 \%$ contre $40,2 \%, 16 \%$ et $46,5 \%$ pour les hommes respectivement [28].

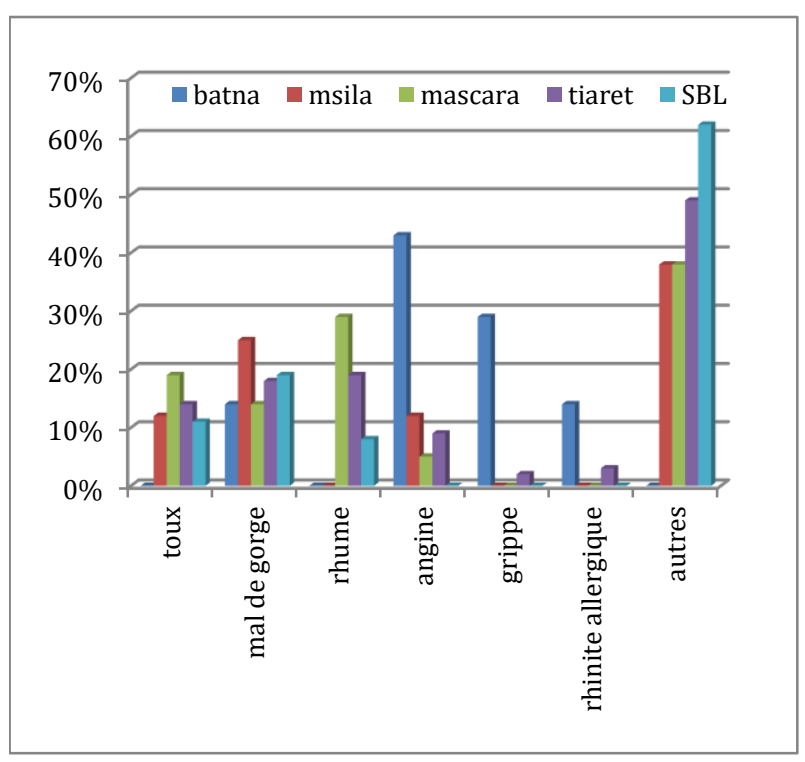

Figure1 : Motifs de demande de l'ATB

Contrairement aux autres villes algériennes étudiées, les hommes constituent la tranche prédominante dans la pratique de l'automédication par les ATBs, à l'exception de M'Sila où les deux sexes sont égaux. Ceci nous laisse penser sur la possibilité d'existence de facteurs sociétaux propres à chaque ville et qu'il serait intéressant de les étudier.

En ce qui concerne la destination des ATBs demandés, la réponse principale était la même quel que soit la ville; le patient étant le demandeur lui-même (des pourcentages allant de $35,1 \%$ à $75 \%$ ) et secondairement pour les enfants dans toutes les villes $(32,4 \%$ à $42,9 \%)$ à l'exception de la ville de M'Sila (0\%). Ces résultats révèlent un usage remarquable des ATBs chez la population pédiatrique.

D'après les données recueillies, la plupart de ceux qui s'adonnent à l'automédication par les ATBs appartiennent à la tranche d'âge 30-44 ans avec des pourcentages : $85,7 \%$ à Batna, 42,8\% à Mascara, 37,5\% à M'sila, 43,8\% à Tiaret et $32,4 \%$ à Sidi-Bel-Abbès. Ce résultat rejoint encore une fois ceux de la littérature par exemple la même tranche d'âge retrouvée en Pologne avec un pourcentage de 49\% [35].

Comparativement aux résultats menés par Napolitano et al. en Italie et Awad et al. au Kuwait, c'est la même tranche d'âge qui est touchée par le phénomène de l'automédication par les ATBs avec des taux de $35,8 \%$ et $40,5 \%$ respectivement [36]. On a constaté que même les sujets âgés pratiquent l'automédication dans certaines régions mais avec un pourcentage minime (4,76\% à Mascara, 1,36\% à Tiaret, 5,4\% à Sidi-Bel-Abbès) du fait que la majorité d'entre eux ont des maladies chroniques et ils sont suivis par au moins un médecin. Ce résultat obtenu est comparable à celui trouvé au Kuwait $(4,9 \%)[33]$; par contre, il est nettement inférieur à celui trouvé en Pologne (27,2\%) [35].

La majorité de notre population cible ont un niveau d'instruction soit secondaire $(42,9 \%$ à Batna, 35,6\% à Tiaret, $40,5 \%$ à Sidi-Bel-Abbès) soit universitaire $(42,9 \%$ à Batna, $28,6 \%$ à Mascara et $50 \%$ à M'sila). Ceci est inférieur aux 
Tableau 5. L'antibiotique demandé sans prescription médicale.

\begin{tabular}{|c|c|c|c|c|c|}
\hline L'ATB demandé & Batna & M'sila & Tiaret & SBA & Mascara \\
\hline Amoxicilline $125 \mathrm{mg}(\mathrm{Sp})$ & & & $01(1.39 \%)$ & & \\
\hline Amoxicilline $250 \mathrm{mg}(\mathrm{Sp})$ & & & $04(5.5 \%)$ & $01(2.7 \%)$ & $03(14.3 \%)$ \\
\hline Amoxicilline $500 \mathrm{mg}(\mathrm{Sp})$ & & & $04(5.55 \%)$ & $03(8.1 \%)$ & $01(4.8 \%)$ \\
\hline Amoxicilline $500 \mathrm{mg}(\mathrm{Gl})$ & & & $01(1.39 \%)$ & $01(2.7 \%)$ & $03(14.3 \%)$ \\
\hline Amoxicilline $1 \mathrm{~g}(\mathrm{Cp})$ & $03(42.9 \%)$ & $01(12.5 \%)$ & $09(12.5 \%)$ & $02(5.4 \%)$ & $02(9.5 \%)$ \\
\hline Amoxicilline+acide clavulanique $100 / 12.5 \mathrm{mg}(\mathrm{Sp}$ ) & $01(14.3 \%)$ & & & $01(2.7 \%)$ & \\
\hline Amoxicilline/ac.clavulanique 228/12.5 mg (Sp) & & & $01(1.39 \%)$ & & \\
\hline Amoxicilline+ acide clavulanique $400 / 62,5 \mathrm{mg}$ (Sp) & & & $01(1.39 \%)$ & & \\
\hline Amoxicilline+acide clavulanique 500/62.5mg (24Cp) & $01(14.3 \%)$ & & & & \\
\hline Amoxicilline+acide clavulanique $1 \mathrm{~g}$ (Sachet) & & $01(12.5 \%)$ & & $01(2.7 \%)$ & \\
\hline Ampicilline 500 mg (Gl) & & & & & $02(9.5 \%)$ \\
\hline Cefalexine $1 \mathrm{~g}(\mathrm{Cp})$ & & & $02(2.78 \%)$ & $01(2.7 \%)$ & \\
\hline Oxacilline 500mg (Gel) & & & $04(5.55 \%)$ & $1(2.7 \%)$ & \\
\hline Oxacilline $500 \mathrm{mg}$ (inj) & & & $01(1.4 \%)$ & & $01(4.8 \%)$ \\
\hline Pénicilline V 1M (Cp) & & $01(12.5 \%)$ & $05(6.94 \%)$ & $03(8.1 \%)$ & $01(4.8 \%)$ \\
\hline Pénicilline V 1.5M (Cp) & & $01(12.5 \%)$ & $02(2.78 \%)$ & & $01(4.8 \%)$ \\
\hline Pénicilline V 0.250M (Sp) & & & $02(2.78 \%)$ & & \\
\hline Pénicilline V 0.400M (Sp) & & & $02(2.78 \%)$ & & \\
\hline Pénicilline G + Benzathine 1.2M (INJ) & & & & $01(2.7 \%)$ & $01(4.8 \%)$ \\
\hline Azithromycine 500mg (Gel) & & & $02(2.78 \%)$ & & \\
\hline Erythromycine $200 \mathrm{mg}(\mathrm{Sp})$ & & & $01(1.39 \%)$ & & \\
\hline Spiramycine $1.5 \mathrm{M}(\mathrm{Cp})$ & $01(14.3 \%)$ & & $03(4.16 \%)$ & $01(2.7 \%)$ & \\
\hline Spiramycine $3 \mathrm{M}(\mathrm{Cp})$ & & $01(12.5 \%)$ & $01(1.39 \%)$ & $01(2.7 \%)$ & \\
\hline Spiramycine $0.375 \mathrm{M}(\mathrm{Sp})$ & & & & $01(2.7 \%)$ & \\
\hline Métronidazole+Spiramycine (Cp) & $01(14.3 \%)$ & $01(12.5 \%)$ & $01(1.39 \%)$ & $01(2.7 \%)$ & \\
\hline Métronidazole 500mg (Cp) & & $01(12.5 \%)$ & $01(1.39 \%)$ & & \\
\hline Doxycycline $100 \mathrm{mg}(\mathrm{Cp})$ & & & $02(2.78 \%)$ & & \\
\hline Nifuroxazide $200 \mathrm{mg}(\mathrm{Gl})$ & & & & $01(2.7 \%)$ & $01(4.8 \%)$ \\
\hline Nitroxoline 100mg (Cp) & & & $02(2.78 \%)$ & & \\
\hline Gentamicine 80mg (inj) & & & $01(1.39 \%)$ & & \\
\hline Ac. Fusidique 2\% (Pd derm) & & & $01(1.39 \%)$ & $01(2.7 \%)$ & \\
\hline Triamcinolone+ néomycine+ nystatine (pd derm) & & $01(12.5 \%)$ & $06(8.33 \%)$ & $09(24.3 \%)$ & \\
\hline Néomycine 1\% (Pd. oph) & & & $05(6.94 \%)$ & $06(16.2 \%)$ & 04 (19\%) \\
\hline Néomycine+triamcinolone (pd oph) & & & $01(1.39 \%)$ & & \\
\hline Norfloxacine (Collyre) & & & & $01(2.7 \%)$ & \\
\hline Déxamèthasone+Néomycine+ Polymyxine B (collyre) & & & $01(1.39 \%)$ & & \\
\hline Rifamycine (gtte auriculaire) & & & $02(2.78 \%)$ & $01(2.7 \%)$ & $01(4.8 \%)$ \\
\hline Niframycine (pulv.nsale) & & & $02(2.78 \%)$ & & \\
\hline Métronidazole+ néomycine+ nystatine (ovules) & & & $01(1.39 \%)$ & & \\
\hline
\end{tabular}

proportions observées au Kuwait $(87,5 \%)$ et au Yémen $(65,8 \%)[28,33]$. En Iran par contre, les universitaires constituent $31,5 \%$ de la population [15]. Le recours à l'automédication par ces deux tranches peut être expliqué soit par leur connaissance soit l'expérience antérieure avec ces molécules. Cette dernière raison peut être renforcée par le résultat obtenu à Mascara où les analphabètes constituent $33,3 \%$ de ceux qui s'automédiquent par les ATBs.

Concernant la situation professionnelle des participants dans notre étude, la majorité appartiennent à la fonction publique tel qu'à Mascara (33,3\%), à M'sila (37,5\%), à Tiaret (42,5\%) et Sidi-Bel-Abbès $(24.3 \%)$ ou à une profession libérale chez les batnéens (42,9\%) contrairement à Mascara où les chômeurs constituent un pourcentage non négligeable $(28,6 \%)$ comparable à celui de Ryad $(31,6 \%)$ [5]. Alors qu'au Kuwait, les chômeurs constituent $40 \%$ [33]. Un pourcentage qui n'est plus loin de celui retrouvé dans une étude italienne réalisée en 2013 (45,8\%) [36].

Pour acheter un ATB sans ordonnance, l'expérience antérieure était la base du choix la plus prédominante: $85,7 \%$ à Batna, $57,1 \%$ à Mascara, $75 \%$ à M'sila, $53,4 \%$ à Tiaret et $51,4 \%$ à Sidi-Bel-Abbès ce qui dépasse les résultats trouvés en Arabie Saoudite (31,6\%), en Ouzbékistan (32,2\%) et en Iran $(11,4 \%)[15,28]$. Au Brésil, d'après Corréa da Silva et al, $72,8 \%$ de ceux qui s'automédiquent se basent sur le conseil d'un ami et de proches [12] alors que chez notre population, cette source représente un pourcentage entre $2,7 \%$ et $6,8 \%$ seulement. Ceci est proche du pourcentage obtenu au Yémen $(7,3 \%)$ [28]. Un taux remarquable de ceux qui demandent conseil aux professionnels de santé a été constaté à Mascara $(45,9 \%)$ ainsi qu'en Ouzbékistan où $45 \%$ de la population enquêtée demande conseil aux pharmaciens pour choisir l'ATB à délivrer sans prescription médicale [28].

Nos statistiques révèlent que 38,1\% à Mascara, 25\% à M'sila s'automédiquent par les ATBs pour des raisons économiques. Cela est la même raison poussant les étudiants au Ghana à s'automédiquer avec un pourcentage de $40,5 \%$ [4] et $26,7 \%$ au Brésil [12] alors que $57,1 \%$ à Batna, 39,7\% à Tiaret et $35,1 \%$ à Sidi-Bel-Abbès ont signalé que la banalisation de la maladie représente le motif essentiel pour ne pas consulter un médecin. Ce sont des pourcentages qui ne sont pas très loin de ceux trouvés au Brésil (44\%) [12]. Suite au manque du temps, $26 \%$ à Tiaret et $25 \%$ à M'sila ont déclaré ne pas avoir assez de temps pour consulter un médecin surtout avec le temps d'attente relativement lent, ce qui les pousse à solliciter les officines pour obtenir les ATBs facilement. C'était la même raison avec le même pourcentage rencontré chez les étudiants brésiliens (26\%) [12] et 17,6\% au Nigeria [32] contrairement au Ghana où le pourcentage était plus élevé $(40,5 \%)[4]$.

$43,2 \%$ de notre population cible à Sidi-Bel-Abbès a d'autres motivations les poussant à l'automédication par les ATBs telles que le manque de confiance en les prescripteurs, ils déclarent toujours obtenir les mêmes prescriptions à chaque nouvelle consultation et la difficulté d'accès aux services hospitaliers. Ce sont les mêmes motivations rencontrées en Iran avec des pourcentages de $0,3 \%$ et $8,9 \%$ respectivement [15] et pour le motif économique, il constitue $0,9 \%$ au Ghana [4] contre $14,3 \%$ à Batna, $25 \%$ à M'sila, 21,9\% à Tiaret, $38,1 \%$ a Mascara et seulement 2,7\% a Sidi-Bel-Abbès.

25\% des participants à M'sila ont déclaré avoir acheté l'ATB 
sans prescription médicale pour traiter le mal de gorge. C'était la même indication retrouvée en Albanie et au Nigéria avec des taux de $29,1 \%$ et $20,3 \%$ respectivement $[34,37]$ contre 10,8\% seulement en Egypte [38]. La toux était l'indication majeure de l'automédication par les ATBs au Yémen et en Arabie Saoudite avec des pourcentages de 41,1\% et $52,4 \%$ respectivement [28] alors qu'elle représente $14,7 \%$ en Albanie [34], $13,7 \%$ à Tiaret, $12,5 \%$ à M'sila, $8,7 \%$ au Nigéria [37], 19\% à Mascara et $10,8 \%$ à Sidi-Bel-Abbès et moins en Egypte avec seulement 1,7\% [38]. Dans 28,6\% des cas à Mascara et $19,2 \%$ à Tiaret, l'ATB était délivré sans ordonnance pour traiter le rhume. Loin de ces pourcentages, le rhume constitue seulement $7,1 \%$ des indications de l'automédication par les ATBs en Albanie [34]. La majorité des participants dans notre étude au niveau des cinq villes algériennes ont déclaré d'autres indications pour l'automédication par les ATBs telles que : la rhinite allergique, la grippe, la fièvre, les angines, les abcès dentaires et même des céphalées! Ce qui explique de plus en plus l'émergence terrible de la résistance bactérienne aux ATBs.

Selon les résultats on y voit clairement la prédominance de la famille des bétalactamines (pénicillines principalement), suivie par les macrolides en deuxième position avec leurs différentes formes et dosages adultes et pédiatriques. L'Amoxicilline est la molécule la plus demandée à Batna $(42,9 \%)$ et Mascara (42,9\%), ceci est retrouvé aussi dans l'étude menée en 2009 à Téhéran en Iran (40\%) [21]. Pour Tiaret et Sidi-Bel-Abbès les pourcentages étaient plus faibles, $18,9 \%$ et $26,3 \%$ respectivement; la même proportion est retrouvée en Egypte (16,3\%) [38] et au Nigeria (17,6\%) [37]. L'Amoxicilline associée à l'acide clavulanique (inhibiteur des bétalactamases), a été plus demandée à Batna $(28,6 \%)$ et M'sila $(12,5 \%)$ contrairement à Tiaret $(2,8 \%)$ et Sidi-BelAbbès $(2,7 \%)$, ces deux dernières se rapprochent du résultat obtenu au Nigeria $(4,4 \%)$ [37].

Pour la Pénicilline, Batna conserve toujours un pourcentage comparable à celui de l'Amoxicilline associée à l'acide clavulanique (25\%), par contre à M'sila, à Sidi-Bel-Abbès et à Mascara les proportions étaient un peu faibles (15,3\%, 11\% et $14,4 \%$ respectivement) mais qui sont comparables au pourcentage retrouvé à Téhéran (13.3\%) [21]. Enfin, la Spiramycine était le macrolide le plus demandé après les pénicillines; les proportions étaient $14,3 \%$ à Batna, $12,5 \%$ à M'sila, $5,6 \%$ à Tiaret et $8,1 \%$ à Sidi-Bel-Abbès; ceci est nettement supérieur à la proportion retrouvée en Egypte $(2,96 \%)[38]$.

Cette pré-enquête nous a permis d'avoir une idée sur le phénomène de l'automédication par les ATBs dans notre société, qui est bien présent; mais malheureusement cinq officines ne sont pas représentatives de la réalité du phénomène ce qui nous amène a projeter de mener une enquête plus approfondie touchant un nombre plus important de pharmacies et de villes algériennes afin de pouvoir estimer l'ampleur réelle de cette pratique.

Elle nous a permis aussi, de voir clairement que l'automédication touche plusieurs classes d'ATBs qu'ils soient à usage systémique (voie orale et parentérale) ou local (pommade ophtalmique, collyre, gouttes auriculaire, gouttes nasales et pommades dermiques) et le plus important, est qu'elle ne fait pas de distinction entre les adultes et les enfants.

Déclaration d'intérêts : les auteurs ne déclarent aucun conflit d'intérêt en rapport avec cet article.

\section{RÉFÉRENCES}

1. Fainzang $S$, L'automédication : une pratique qui peut en cacher une autre. Anthropologie et Sociétés, 2010 ; 34(1) : 115-133.

2. Bertoldi A D, Camargo LA, Silveira MPT, et al. Self-Medication among adolescents aged 18 years: the 1993 Pelotas (Brazil) birth cohort study. Journal of Adolescent Health xxx (2014) 1-7

3. 3Mohanna M. Self-medication with antibiotic in children in Sana'a City, Yemen. OMJ.2010; 25: 41-43

4. Donkor ES, Tetteh-Quarcoo PB, Nartey P, Agyeman IO. SelfMedication Practices with antibiotics among tertiary level students in Accra, Ghana: A cross-sectional study. Int J Environ Res Public Health. 2012; 9(10): 3519-3529.

5. Alghanim SA. Self-medication practice among patients in a public health care system. EMHJ.2011; 17 (5):409-416.

6. El Ezz NF, Ez-Elarab HS. Knowledge, attitude and practice of medical students towards self medication at Ain Shams University, Egypt.J prev med hyg. 2011; 52: 196-200.

7. Abasaeed A, Vlcek J, Abuelkhair M, Kubena A. Self-medication with antibiotics by the community of Abu Dhabi Emirate, United Arab Emirates.J Infect Dev Ctries. 2009; 3(7):491-497.

8. Umair M. Self medications. jphbs.2012; 1(4):145-146.

9. Abobotain $\mathrm{AH}$, Sheerah HA, Alotaibi FN, et al. Socio-demographic determinants of antibiotic misuse in children. A survey from the central region of Saudi Arabia. Saudi Med J. 2013; 34 (8): 832-840.

10. Heidarifar R, Koohbor M, K Mansourabad M, Mikaili P, Sarahroodi S. Self-medication with Antibiotics among Iranian Population in Qom State. J sir journal.2013; 2 (4): 785-789.

11. Papakosta M, Zavras D, Niakas D. Investigating factors of self-care orientation and self medication use in a Greek rural area. Rural and Remote Health.2014; 14: 23-49.

12. Silva MGCD, Soares MCF, Muccillo-baisch AL. self-medication in university students from the city of Rio Grande, Brazil. BMC.2012; 12:339.

13. Gutema GB, Gadisa DA, Kidanemariam ZA, et al. Self-Medication Practices among Health Sciences Students: The case of Mekelle University.Journal of Applied Pharmaceutical Science.2011; 01 (10): 183-189.

14. Sapkota AR, Coker ME, Goldstein RER, Atkinson NL, Sweet SJ, Sopeju $P O$. Self-medication with antibiotics for the treatment of menstrual symptoms in south west Nigeria: a cross-sectional study.BMC Public Health. $2010 ; 10: 610$.

15. Jalilian F, Hazavehei SMM, Vahidinia AA, Jalilian M, Moghimbeigi A. Prevalence and related factors for choosing self-medication among pharmacies visitors based on health belief model in Hamadan province, west of Iran. J Res Health Sci.2013; 13(1): 81-85.

16. Shoaib MH, Yousuf RI, Anjum F, et al. Survey based study on the use of non-prescription drugs among pharmacists and non-pharmacists. Afr. J. Pharm. Pharmacol.2013; 7(38): 2652-2656.

17. Sawalha AF, A descriptive study of self-medication practices among Palestinian medical and non medical university students. Research in Social and Administrative Pharmacy.2008; 4:164-172.

18. Al-azzami SI, Al-husein BA, alzoubi F, Massadeh MM, Al-Horani MAS. Self medication with antibiotics in Jordanian population. IJOMEH. 2007;20(4):373 - 380 .

19. Agence Française de Sécurité Sanitaire et des Produits de Santé. L'antibiotique, un médicament pas comme les autres. Document à destination des professionnels de santé. France : afssaps ; 2009.

20. Mainous AG, Everett CJ, Post RE, Diaz VA, Hueston WJ, Availability of Antibiotics for Purchase without a Prescription on the Internet. Annals of family medicine.2009; 7 (5):431-435. 
21. Sarahroodi S, Arzi A. Self medication with antibiotics, is it a problem among Iranian college students in Tehran?. J.Biol.Sci. 2009; 9(8): 829-832.

22. Sawair FA, Baqain ZH, Abu Karaky A, Abu Eid R. Assessment of SelfMedication of antibiotics in a Jordanian Population. Med Princ Pract. 2009;18:21-25.

23. Barah F, Gonçalves V. Antibiotic use and knowledge in the community in Kalamoon, Syrian Arab Republic: a cross-sectional study. EMHJ. 2010; 16(5)

24. Jassim AM. In-home drug storage and self-medication with antimicrobial drugs in Basrah, Iraq. OMJ. 2010; 25: 79-87.

25. Le TH, Ottosson E, Nguyen TKC, Kim BG, Allebeck P. Drug use and self-medication among children with respiratory illness or diarrhea in a rural district in Vietnam: a qualitative study. Journal of Multidisciplinary Healthcare. 2011;4:329-336.

26. Ahmad H, Sherwani SK, Nisar W, et al. Self-medication with antibiotics: general perspective among people in peshawar and mardan (Two most populated cities of khyber pukhtoonkhwaPakistan). IJPSR. 2013; 4(5): 1809-1814.

27. Gastelurrutia MA, Larranaga B, Garay A, Echevest FA, FernandezLlimos F. Impact of a program to reduce the dispensing of antibiotics without a prescription in Spain. Pharmacy Practice.2013; 11(4): 185190.

28. Belkina T, Al Warafi A, Eltom EH, et al. Antibiotic use and knowledge in the community of Yemen, Saudi Arabia and Uzbekistan. J Infect Dev Ctries. 2014; 8(4):424-429.

29. Bennadi D. Self-medication: a current challenge. JBClinPhrm.2014; 5(1): 19-23
30. Pan H, Cui B, Zhang D, Farrar J, Law F, Ba-Thein W. Prior Knowledge, Older Age, and Higher Allowance Are Risk Factors for SelfMedication with Antibiotics amongUniversity Students in Southern China. PLoS ONE. $2012 ; 7(7)$.

31. Yousef AM, Al-Bakri AG, Bustanji Y, Wazaify M. Self-Medication Patterns in Amman, Jordan. Pharm World Sci. 2008; 30:24-30.

32. Auta A, Omale S, Folorunsho TJ, David S, Banwat SB. Medecine vendors : self medication practices and medecine knowledge. $\mathrm{N} \mathrm{Am}$ J Med Sci. $2012 ; 4(1): 24-28$

33. Awad Al, Aboud EA. Knowledge, attitude and practice towards antibiotic use among the Public in Kuwait. PLoS ONE. $2015 ; 10(2)$ : e0117910.

34. Jorgji K, Bebeci E, Apostoli P, Apostoli A. Evaluation of use of antibiotics without prescription among young adults in Albania case study : Tirana and Fier Discrit. Hippokratia. 2014 ; 18(3) : 217-220.

35. Muas M, Krajewski J, Nocun M, Godycki-Cwirko M. A survey of patient behaviours and beliefs regarding antibiotic self-medication for respiratory tract infections in Poland. Arch Med Sci. 2013 ; 9 (5) : 854-857.

36. Napolitano F, Izzo MT, Di Giuseppe G, Angelillo IF. Public knowledge, attitudes and experience regarding the use of antibiotics in Italy. Plos One. 2013 ; 8 (12) : e 84177.

37. Fadare JO, Tamuno I. Antibiotic self-medication among university medical undergraduates in Northen Nigeria. Journal of Public Health and Epidemiology. $2011 ; 3(5)$ : 217-220.

38. Sabry NA, Farid SF, Dawoud DM. Antibiotic dispensing in Egyptian community Pharmacies : An observational study. Research in Social and Administrative Pharmacy. 2013 ; 1-17
Cet article a été publié dans le « Batna Journal of Medical Sciences » BJMS, l'organe officiel de "l'association de la Recherche Pharmaceutique - Batna»

Le contenu de la Revue est ouvert "Open Access » et permet au lecteur de télécharger, d'utiliser le contenu dans un but personnel ou d'enseignement, sans demander l'autorisation de l'éditeur/auteur.

Avantages à publier dans BJMS :

- Open access : une fois publié, votre article est disponible gratuitement au téléchargement

- Soumission gratuite : pas de frais de soumission, contrairement à la plupart des revues « Open Access "

- Possibilité de publier dans 3 langues : français, anglais, arabe

- Qualité de la relecture : des relecteurs/reviewers indépendants géographiquement, respectant l'anonymat, pour garantir la neutralité et la qualité des manuscrits.

Pour plus d'informations, contacter BatnaJMS@gmail.com ou connectez-vous sur le site de la revue : www.batnajms.com

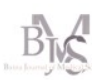

Batna Journal of

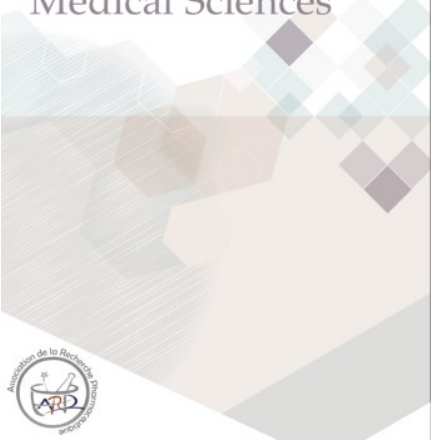




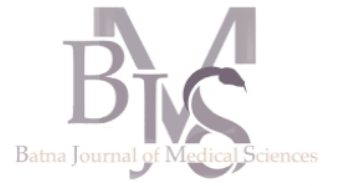

Département de Médecine, Université Hadj Lakhdar, Batna, Algérie

Correspondance à :

Samy SLIMANI

slimani@dr.com

DOI :https://doi.org/10.48087/ B]MScb.2015.2108

Il s'agit d'un article en libre accès distribué selon les termes de la licence Creative Commons Attribution International License (CC BY 4.0), qui autorise une utilisation, une distribution et une reproduction sans restriction sur tout support ou format, à condition que l'auteur original et la revue soient dûment crédités.

\section{Pour citer l'article :}

Slimani S. Brèves de l'IOFESCEO 2015. Maladies systémiques : lupus, Sjögren et vascularites. Batna J Med Sci 2015;2(1):36-38. https://doi.org/10.48087/BJMS

\section{Brèves du Congrès de l'IOF-ESCEO 2015}

\section{Congrès mondial de l'ostéoporose, de l'arthrose et des affections musculosquelettiques}

\section{Samy Slimani}

\section{Résumé}

Le congrès mondial de l'arthrose et de l'ostéoporose, baptisé ainsi depuis cette année, a eu lieu cette année à Milan en Italie, co-organisé par I'International Osteoporosis Foundation (IOF) et l'European Society for Clinical and Economic Aspects of Osteoporosis (ESCEO). Le congrès a réuni plus de 3200 participants qui ont assisté aux différentes sessions du congrès, très riche quantitativement avec plus de 600 abstracts présentés, et qualitativement avec l'inclusion de plusieurs sessions sur l'arthrose, l'ostéoporose, les pathologies musculaires et les pathologies du sujet âgé. Je vous présente une sélection de certains travaux marquants de ce congrès.

Mots clés : Congrès mondial, ostéoporose, arthrose, affections musculosquelettiques.

Efficacité et tolérance au long terme de l'ODANACATIB, anti-Kathepsine $\mathrm{K}$, dans l'ostéoporose féminine

\section{(OC1, par MR McClung).}

Il s'agit des résultats d'une étude de phase III, étude LOFT, randomisée, en double aveugle, évaluant l'efficacité et la tolérance au long terme de ce nouvel agent, qui inaugure une nouvelle classe thérapeutique d'antirésorbeurs anti-ostéoporotiques, ciblant la cathepsine K qui est une enzyme essentielle au fonctionnement de l'ostéoclastogenèse. Ont été inclus 16.713 patientes ostéoporotiques $(<-$ 2,5) âgées de plus de 65 ans, randomisées en groupe placebo et groupe odanacatib. Les deux bras avaient reçu du calcium et de la vitamine D. Le critère de jugement principal était la survenue de nouvelles fractures vertébrales. La durée moyenne de suivi était de 35 mois. La prise d'odanacatib était associée à une réduction de $54 \%$ du risque de nouvelles fractures vertébrales, mais aussi à une réduction de $47 \%$ du risque de fractures du fémur et de $23 \%$ du risque de fractures non vertébrales. La tolérance a été très bonne.

Switch thérapeutique après traitement par ROMOSOZUMAB, une réponse à l'horizon?

(OC3) MR McClung et al, avaient présenté les résultats d'une séquence thérapeutique faite de 2 ans de traitement par ROMOSOZUMAB, suivi d'une rotation d'un an par le DENOSUMAB, chez les femmes présentant une ostéoporose post-ménopausique. Le ROMOSOZUMAB est un anticorps monoclonal qui inhibe la sclérostine, protéine exprimée par les ostéocytes et impliquée dans l'inhibition de l'action des ostéoblastes. Il avait prouvé une efficacité et une bonne tolérance chez la femme ménopausée. Le DENOSUMAB est un inhibiteur du RANKL, protéine clé du processus de résorption osseuse. L'étude de cette séquence thérapeutique avait inclus 419 femmes ménopausées avec un T-score compris entre 2,0 et $-3,5$. Le ROMOSOZUMAB avait entrainé une amélioration des chiffres de DMO à 1 et 2 ans, comparé au placebo. Dans le groupe ayant bénéficié d'une rotation vers le DENOSUMAB, la poursuite du gain des chiffres de DMO était nette, alors que dans le bras placebo, la DMO est presque retournée aux valeurs de base, avant le début du traitement par ROMOZOZUMAB (figure 1). Les auteurs concluent que la séquence de 2 ans d'anticorps anabolisant puis 1 an d'anticorps antirésorbeur était bénéfique et bien tolérée.
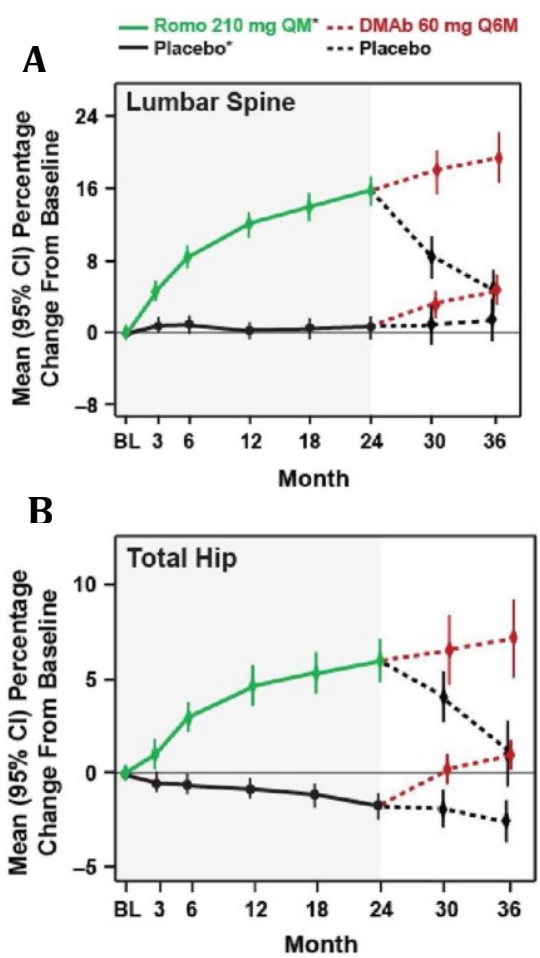

Figure 1 : évolution de la DMO sous traitement. A. au niveau du rachis lombaire et $\mathbf{B}$. au fémur. 


\section{Vitamine D et grossesse.}

\section{Par RJ Moon (OR6)}

Le dosage de la vitamine $\mathrm{D}$ est devenu une procédure de routine chez la femme enceinte, au début de sa grossesse. il est cependant inconnu si des changements dans le taux de la vitamine D surviennent durant la grossesse, passant d'un état à un autre, plus alarmant ou plus rassurant, d'un trimestre à l'autre. Plus de 2000 femmes de la "Southampton Women's Survey » ont été incluses et suivies durant leur grossesse, avec un dosage régulier de la vitamine D sérique. Globalement, les taux sont restés stables tout au long de la grossesse, sans aggravation en fin de grossesse. Le principal déterminant de la variation dans le temps était l'introduction ou l'arrêt de la supplémentation en vitamine $\mathrm{D}$. Un autre facteur associé à la baisse de la vitamine $\mathrm{D}$ était l'augmentation importante du poids.

\section{Arthrose et mortalité, un lien ? Données d'une cohorte Suédoise (OC12) Par Kluzek S et al.}

Plusieurs études avaient suggéré un lien entre la survenue d'une arthrose et la surmortalité. La cohorte Suédoise de Chingford avait essayé de répondre à cette question. Il s'agit d'une cohorte de sujets suivis pendant 23 ans. Ont été recherchées la présence/absence d'une arthrose des genoux et des mains, et le caractère douloureux ou pas de cette arthrose. Pour la définition radiographique, un score de Kellgren et lawrence de 2 et plus a été exigé. La mortalité a été évaluée à partir du registre des décès arrêté en 2014. Les éventuelles associations avec la mortalité ont été évaluées en utilisant un modèle Cox de régression. Au total, sur les 821 femmes suivies, 163 décès sont survenus, le groupe de gonarthrose douloureuse avait un risque plus élevé de mortalité (HR $=1,44$, IC 95\% 0,99-2,08). Les auteurs concluent que seule la gonarthrose " clinico-radiologique » était associée à une surmortalité. L'arthrose digitale, douloureuse ou pas n'était pas associée à une surmortalité, ainsi que la gonarthrose radiographique asymptomatique. L'explication serait que les femmes présentant une gonarthrose symptomatique à l'âge moyen ont plus souvent des changements métaboliques (obésité...) que les femmes non arthrosiques, ce qui augmente le risque de développer des événements cardiovasculaires et entrainer par conséquent une surmortalité.

\section{Recommandations de 2015 de la prise en charge de la gonarthrose, de l'ESCEO}

\section{Par 0 Bruyère}

Après les conclusions alarmistes de l'OARSI 2014 concernant la viscosupplémentation, le très récentes recommandations de l'ESCEO 2015 dans la prise en charge de la gonarthrose, redonnent une place à la viscosupplémentation, chez les gonarthrosiques en échec à un traitement symptomatique de première intention, ou chez les patients présentant une contre indication ou une intolérance aux AINS/antalgiques. L'intégralité des recommandations est téléchargeable et en accès libre en suivant le lien :

www.semarthritisrheumatism.com/article/S00490172(14)00108-5/pdf
Vitamine D et sclérodermie, encore un effet bénéfique.

\section{P282, Di Donato E et al.}

La vitamine $\mathrm{D}$ a été associée à plusieurs maladies systémiques, dont la sclérodermie. L'hypovitaminose D était accompagnée d'une fibrose cutanée plus étendue. Cette équipe italienne avait pour objectif d'évaluer l'association entre le taux sérique de la vitamine $D$ et la survenue d'ulcères digitaux, et si oui, de rechercher un éventuel effet bénéfique à supplémenter les patients en vitamine D. 87 patients ont été inclus, dans 3 centres. Les ulcères digitaux (UD) survenus dans les 6 derniers mois ont été enregistrés, de même que le taux sérique de vitamine D. Résultats : le taux d'ulcères digitaux a été plus élevé chez les sujets avec une vitamine $\mathrm{D}<$ $10 \mathrm{ng} / \mathrm{ml}(50 \%)$ que chez les patients dont le taux est $>30$ $\mathrm{ng} / \mathrm{ml}(14 \%, \mathrm{p}<0,05)$ (figure 2). Cependant, une tendance non significative vers l'amélioration a été observée sous supplémentation en vitamine D. Des études prospectives thérapeutiques sont requises afin d'évaluer avec précision la place de la supplémentation en vitamine $\mathrm{D}$ dans la prévention des UD chez les patients atteints de sclérodermie.

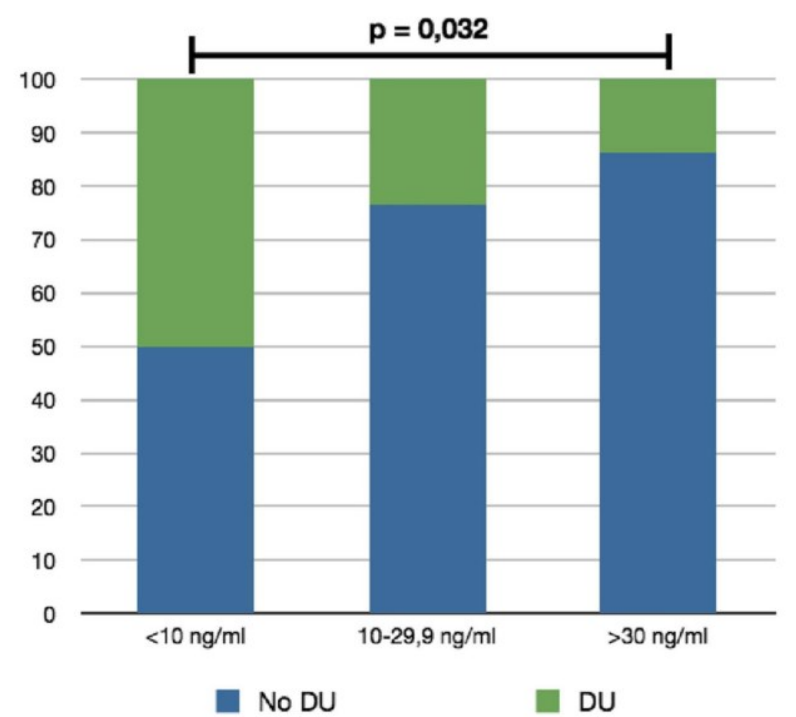

Figure 2: survenue des ulcères digitaux (DU) en fonction du taux sérique de vitamine $\mathrm{D}$.

\section{Méta-analyse du TBS (trabecular bone score) et de ses interactions avec le FRAX.}

\section{oC18, Kanis JA et al.}

Le TBS est un indice permettant d'évaluer la microarchitecture osseuse, il est réalisé lors d'un examen de DMO. L'objectif de cette étude était de monter une métaanalyse afin de déterminer si le TBS permettait de prédire la survenue de fracture, indépendamment du FRAX. Les données de 17.809 patients ont été poolées. Le suivi médian des patients était de 6,5 ans. La relation entre le TBS et le FRAX et le risque fracturaire était évaluée en utilisant un modèle de régression de Poisson. Le gradient de risque (GR) pour une fracture majeure était de 1,44 (IC 95\%: 1,35-1,53), ceci est resté significatif après ajustement au FRAX (GR 1,32; IC 95\% : 1,24-1,41). En conclusion, le TBS est un élément prédictif de survenue de fractures, de façon indépendante du FRAX. 


\section{Evaluation des marqueurs de sarcopénie}

\section{C25, Cavalier E et al.}

Les auteurs avaient évalué une analyse de performance de plusieurs marqueurs sériques de sarcopénie : IRI, MYO, AA, PIINP, OGN et TMEM, sur 120 sujets sains (60 hommes, 60 femmes non ménopausées). Pour rappel, La sarcopénie est un syndrome gériatrique se caractérisant dans un premier temps par une diminution de la masse musculaire qui en s'aggravant sera à l'origine d'une détérioration de la force musculaire et des performances physiques. Elle est associée à un risque de chute et de fracture. Résultats : certains marqueurs n'étaient pas stables dans le temps, d'autres instables lors de la conservation, les auteurs suggèrent que seuls trois paramètres (PIINP, Myostatine et Activine A) avaient satisfait les standards de qualité et pourraient bénéficier d'études ultérieures de faisabilité en pratique clinique.

\section{La longueur de l'axe du fémur est un facteur de risque indépendant de survenue de fractures.}

\section{C27, Leslie WD et al.}

Certaines études avaient suggéré que la longueur excessive de l'axe du fémur était associée à une augmentation du risque de fracture chez les femmes, mais sans véritable analyse ajustée à la DMO et au FRAX. Une étude du registre canadien de Manitoba avait tenté de répondre à cette question. Il s'agit de 4.738 hommes et 50.420 femmes âgés de 40 ans et plus, ayant bénéficié d'un recueil des paramètres démographiques, cliniques, DMO et FRAX et ont été suivis. Les hospitalisations ultérieures pour une fracture du fémur ont été notées. Durant les 6,2 ans de suivi, 70 hommes et 1020 femmes avaient présenté une fracture du fémur. La longueur de l'axe du fémur était associée, indépendamment des autres paramètres, à la survenue de fracture, avec une aire sous la courbe de 0,58 $(0,51-0,65)$ chez les hommes et de 0,60 $(0,51$ 0,65 ) chez les femmes. Conclusion : que ça soit chez l'homme ou chez la femme, la longueur de l'axe du fémur est associée à la survenue de fracture de la hanche, indépendamment du FRAX et de la DMO.

Effets osseux favorables du régime sans gluten chez les femmes atteintes d'une maladie coeliaque.

\section{C29, Zanchetta et al.}

La microarchitecture osseuse trabéculaire et corticale est détériorée chez les femmes atteintes d'une maladie coeliaque, ceci a été prouvé sur des études utilisant le HR-pQCT (scanner haute résolution). Les effets du régime sans gluten sur ces paramètres restent inconnus. Les auteurs avaient étudié ce paramètre chez des femmes atteintes d'une maladie cœliaque, un an après l'initiation d'un régime sans gluten. 26 femmes ont été évaluées. La microarchitecture trabéculaire au radius et au tibia a été améliorée d'environ 8\% (figure 3). Les auteurs concluent à l'amélioration de la microarchitecture, en plus de l'amélioration de la densité osseuse, chez les femmes respectant leur régime de maladie cœliaque.
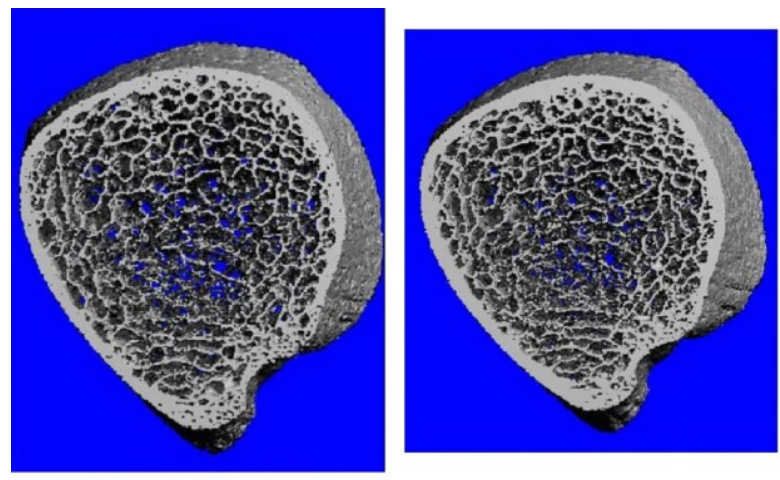

Figure 3: scanner ultra-haute résolution. à droite après régime, comparativement à la figure à gauche avant régime sans gluten

\section{L'ODANACATIB chez l'homme.}

\section{C33, Chapurlat RD et al.}

L'ostéoporose est moins fréquente chez l'homme mais reste une importante source de morbi-mortalité, en plus de son coût élevé en termes de dépenses de santé. L'ODANACATIB (ODC) a été évalué dans un essai randomisé de phase III chez les hommes atteints d'une ostéoporose, sur une durée de 24 mois. Ont été inclus 292 hommes âgée entre 40 et 95 ans, atteints d'une ostéoporose primitive ou secondaire à un hypogonadisme, T-score entre $-2,5$ et $-4,0$. Deux bras, ODC et placebo, recevant en plus une supplémentation vitaminocalcique. Le critère de jugement primaire était l'amélioration de la DMO au rachis lombaire. L'ODANACATIB était efficace et bien toléré, avec une amélioration de la DMO au rachis lombaire et au fémur qui étaient comparables à celle observée chez la femme.

\section{Conclusion}

Congrès riche, sans nouveauté majeure, mais qui a permis une meilleure compréhension de l'épidémiologie de l'ostéoporose et de la sarcopénie, et une meilleure codification de la prise en charge thérapeutique. Deux temps forts : l'élaboration de recommandations ESCEO de la prise en charge de la gonarthrose, et l'arrivée prochaine de deux nouvelles classes thérapeutiques dans l'ostéoporose (anticathepsine K et anti-sclérostine).

Cet article a été publié dans le "Batna Journal of Medical Sciences » BJMS, I'organe officiel de "I'association de la Recherche Pharmaceutique - Batna »

Le contenu de la Revue est ouvert « Open Access » et permet au lecteur de télécharger, d'utiliser le contenu dans un but personnel ou d'enseignement, sans demander l'autorisation de l'éditeur/auteur. 


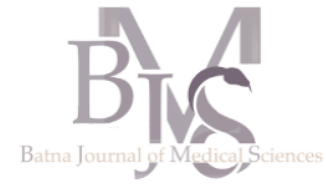

\section{Correspondance à :}

Ghania BELAALOUI

contact genetique@yahoo.fr

DOI :https://doi.org/10.48087/BI MStfe.2015.2109

Il s'agit d'un article en libre accès distribué selon les termes de la licence Creative Commons Attribution International License (CC BY 4.0), qui autorise une utilisation, une distribution et une reproduction sans restriction sur tout support ou format, à condition que l'auteur original et la revue soient dûment crédités.

\section{EDITORIAL}

\section{La génétique humaine : plus qu'un simple engouement, une nécessité !}

Depuis l'élaboration des premières lois de l'hérédité par Gregor Mendel vers la fin du 19ème siècle, passant par la découverte, en 1953, de la structure de l'ADN, support de cette hérédité, l'enthousiasme des chercheurs et des médecins pour l'exploration génétique en pathologie humaine n'a pas cessé de croître, avec une accélération encore plus prononcée depuis l'annonce, en 2003, de la fin du séquençage du génome humain entier. En effet, l'implication des facteurs génétiques en pathologie humaine est indéniable, qu'il s'agisse de certaines maladies rares, souvent lourdes et handicapantes, ou bien de maladies multifactorielles et largement répondues qui constituent des problèmes de santé publique (maladies chroniques non transmissibles : cancer, diabète, hypertension artérielle, asthme,etc.).

Ainsi, la meilleure connaissance des profils génétiques de ces maladies a permis le développement de tests génétiques précieux permettant le diagnostic, le dépistage et/ou le suivi de certaines d'entre elles. Ceci a été rendu possible grâce à l'utilisation des outils de biologie moléculaire et de cytogénétique dont l'apport en pathologie humaine est inestimable. La recherche des mutations $B C R-A B L$ dans un but de diagnostic et de suivi de la leucémie myéloïde chronique (LMC), ou celles du proto-oncogène RET dans un but de réaliser des thyroïdectomies prophylactiques, de même que les tests de dépistage prénataux ou préimplantatoires de certaines maladies génétiques comme la trisomie 21, sont des exemples, parmi tant d'autres, qui peuvent être cités dans ce contexte.

De plus, certaines pathologies multifactorielles ont bénéficié du développement de thérapies dites personnalisées (ou plus correctement, des thérapies de précision, le traitement d'un patient étant pratiquement toujours personnalisé par le thérapeute!). Elles sont basées sur la recherche de certaines mutations chez chaque patient. Ces thérapies visent une meilleure efficacité avec des effets secondaires amoindries. C'est le cas, notamment, de certains inhibiteurs de récepteurs de tyrosines kinases comme l'Imatinib dans la LMC ou le Crizotinib dans le cancer du poumon non à petites cellules, ou bien des anti-récepteurs à l’EGF (Panitumumab ou Cetuximab) dans les cancers colorectaux et pulmonaires. D’un autre côté, certaines thérapies qui, à la base, n'étaient pas considérées comme des thérapies ciblées, le sont devenues par la recherche des meilleurs répondeurs, 'pharmacologiquement' parlant, et ceci grâce aux progrès de la biologie moléculaire qui permettent de détecter ces derniers. On citera, à titre d'exemple, l'intérêt d'étudier le gène CYP2C19 lors de la prescription du Clopidogrel dans les maladies coronariennes. Il ne faudrait pas oublier également que la recherche de certaines mutations, non pas dans l'ADN des patients mais dans celui de certains agents pathogènes qui les ont agressés, comme c'est le cas avec le virus de l'hépatite C, a permis un meilleur ciblage de ces derniers.

Outre ce que l'on vient d'exposer quand l'utilisation des données génétiques dans le diagnostic, le dépistage, le suivi ou le meilleur ciblage thérapeutique, la possibilité de manipuler l'ADN humain à des fins thérapeutiques s'est montrée comme une piste d'étude très attrayante. Ainsi est née la thérapie génique qui, contrairement à ce qu'on pourrait le penser, n'est plus considérée comme une thérapie futuriste mais bel et bien une réelle possibilité thérapeutique pour certaines maladies rares comme le déficit immunitaire combiné sévère lié à l'X (X-SCID), l'adrénoleucodystrophie liée à l'X, l'hémophilie $\mathrm{B}$, ou bien même une maladie multifactorielle plus répondue comme la maladie de Parkinson.

Aussi, on n'omettra surtout pas de noter, qu'au-delà des études génétiques, de plus en plus d'enthousiasme se développe autour de l'« épigénétique » : une possibilité très séduisante de modifier ce que l'on pensait, jusque-là, être l'information immuable et la prédestinée inévitable portée par le génome humain. En effet, les modifications épigénétiques font partie des moyens via lesquels les facteurs environnementaux exercent un effet sur la prédisposition à certaines maladies, leur développement et leur devenir dans le temps, ce qui ouvre des perspectives très intéressantes quant à leur utilisation à des fins thérapeutiques ou bien même prophylactiques.

En Algérie, l'état des lieux dans le domaine de la génétique humaine est loin d'être satisfaisant, et bien qu'il suscite un intérêt croissant parmi les praticiens, beaucoup reste encore à faire dans ce domaine qui est devenu une vraie nécessité et non pas un simple phénomène 'en vogue' dans la médecine. En effet, la fréquence des maladies génétiques dites 'rares', causée par l'important taux de consanguinité dans notre société, et celle des maladies multifactorielles, finissent par créer des problèmes de santé publique dont les répercussions, à la fois médico-sociales et économiques, sont énormes. Les études génétiques et épigénétiques chez l'Homme progressent à un rythme sans précédent, et la mise à jour de nos connaissances dans ce domaine est indispensable. De plus, l'instauration des investigations génétiques pratiques est une autre nécessité qui s'impose inéluctablement, et œuvrer dans le sens de son développement est devenu également un vrai devoir afin d'offrir à nos patients ce qui existe de mieux pour leur santé !

\section{Pour citer l'article :}

Belaaloui G. La génétique humaine : plus qu'un simple engouement, une nécessité ! Batna J Med Sci 2015;2(1):39. https://doi.org/10.48087/BJMS tfe.2015.2109
Pr Ghania Belaaloui Faculté de Médecine, Université Hadj Lakhdar, Batna - Algérie 


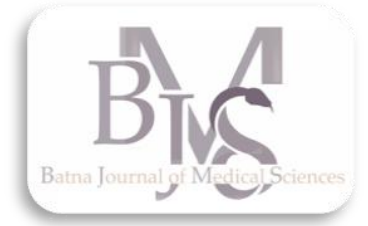

Cabinet libéral de Pédiatrie, 35 Rue De Mostaganem, Oran Algérie

\section{Correspondance à :}

Abdelmadjid BENMANSOUR benmansour_b@yahoo.com

\section{Les mucopolysaccharidoses}

\section{Mucopolysaccharidoses}

\section{Abdelmadjid Benmansour}

\section{RÉSUMÉ}

Les Mucopolysaccharidoses (MPS) sont des maladies héréditaires dues à un défaut enzymatique à l'origine de dépôts de glycosaminoglycanes (mucopolysaccharides) au niveau des lysosomes. Ces dépôts vont se retrouver au niveau de tous les organes de façon plus ou moins importante avec des manifestations plus ou moins précoces et plus ou moins parlantes selon le taux de l'activité résiduelle au niveau des organes atteints.Il y a 7 différents types de mucopolysaccharidoses; Certains signes cliniques sont retrouvés en commun chez la plupart de ces maladies en particulier les organomégalies et l'atteinte osseuse. D'autres sont plus spécifiques chez certaines avec une atteinte cérébrale prédominante telle la MPS III (maladie de San Filippo).Elles font l'objet de très nombreuses recherches et des traitements sont possibles avec des résultats encourageants surtout si le traitement est entrepris précocement. Il y a des traitements adjuvants : physiothérapie, médicamenteux, chirurgie cardiaque, oculaire selon le cas. Il existe un traitement spécifique : la greffe de moelle pour certaines des MPS, et un traitement de substitution enzymatique également pour certaines MPS.Plus récemment, des molécules chaperonnes ont été utilisées pour obtenir une augmentation de l'activité résiduelle et dernièrement un traitement par thérapie génique est en cours dévaluation dans plusieurs centres.

Mots clés : mucopolysaccharidoses ; maladies lysosomales; déficit enzymatique; atteinte multiviscérale ; atteinte cérébrale.

\begin{abstract}
Mucopolysaccharidoses (MPS) are hereditary diseases due to an enzyme deficiency, leading to deposits of glycosaminoglycanes (mucopolysaccharides), in lysosomes. These deposits will be found in all organs in a more or less important way, with more or less early and more or less severe clinical manifestations according to the level of the residual activity in the involved organs. There are 7 different types of mucopolysaccharidoses; some types are found in common in the majority of the diseases, especially the bone involvement and organomegaly. Others are more specific in some types, with a predominant cerebral involvement like in the MPS III (San Filippo disease). These conditions are subject to many research studies and treatments are possible with encouraging results, mostly if the treatment is early initiated. Other adjunctive treatments may be used, such as physiotherapy, symptomatic drugs and heart and eyes surgery. A specific treatment is available: the bone marrow transplant for some types of MPS, and the enzyme substitution in others. Recently, chaperone molecules have been used in order to obtain an increase in the residual activity, and more recently, gene therapy is being evaluated in many centres.
\end{abstract}

Keywords: mucopolysacharidoses; lysosomal diseases; enzyme deficiency; multiorgan deficiency; cerebral involvement.

\section{Pour citer l'article :}

Benmansour A.

Mucopolysaccharidoses. Batna J Med Sci 2015;2:4044.
داء عديد السكاريدات المخاطي
الملخص: المديد السكاريدات المخاطي هو مر ض ور اثثي ناجم عن خلل انزيمي يسبب ترسبات الجلو كوز امين جليكان (عديد السكار يدات

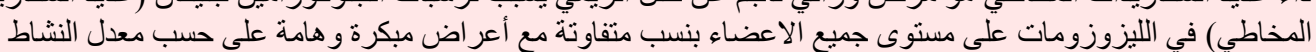

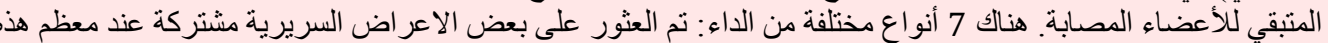

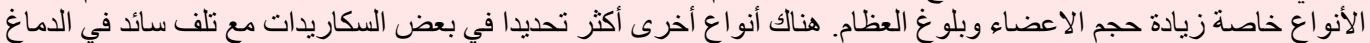

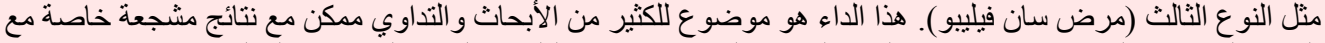

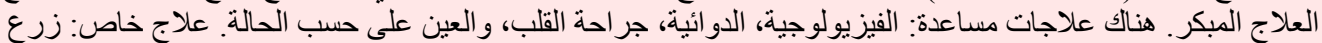

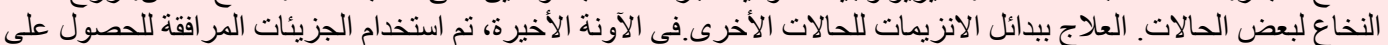

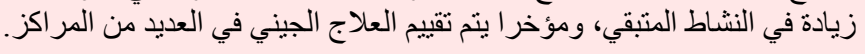

الكلمات المفتاحية: داء عديد السكاريدات المخاطي، داء الليزوزومات، نقص الانزيمات، اصابة أعضاء متعددة، اصابة الدماغ 


\section{DÉFINITION}

Ce sont des maladies héréditaires rares à transmission autosomique récessive à l'exception de la maladie de Hunter qui se transmet selon le mode lié à l'X [1].La plupart sont multisystémiques, même si l'atteinte d'un système peut être prédominante.Elles sont dues à un déficit d'une enzyme lysosomale impliquée dans la dégradation des glucosaminoglycanes (Héparane sulfate, Kératane sulfate, Dermatane sulfate et Chondroïtine sulfate) [1]. Ces glucosaminoglycanes proviennent elles-mêmes de la dégradation de protéoglycanes qui sont des constituants importants de la matrice extracellulaire (sauf l'acide hyaluronique) et du tissu conjonctif de tout l'organisme (figure 1).
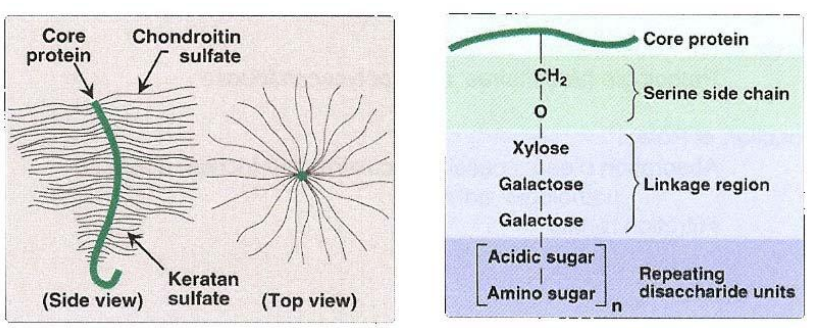

Figure 1 : Schémas extraits du cours de biochimie des glucides pour CPEM Pr. André Le Treut de la Faculté de Médecine de Rennes avec son aimable permission[2].

N'étant pas dégradés, ces éléments vont s'accumuler au niveau des lysosomes et être ainsi à l'origine d'un dysfonctionnement cellulaire, tissulaire et enfin de tous les organes. Les processus physiopathologiques sont encore incomplètement compris et font l'objet de recherche poussée[3].Dans toutes les Mucopolysaccharidoses on retrouve des formes atténuées et des formes sévères. Il ya un continuum entre toutes ces formes (Tableau 1).

Tableau 1 : Classification des mucopolysaccharidoses (MPS) [4]

\section{MPS I : maladie de Hurler, Hurler Scheie, Scheie}

MPS II : maladie de Hunter

MPS III : maladie de San Filippo qui comprend 4 types : A,B,C,D

MPS IV : maladie de Morquio

MPS VI : maladie de Maroteaux Lamy

MPS VII : maladie de Sly

MPS IX : déficit en hyaluronidase

\section{MANIFESTATIONS CLINIQUES COMMUNES AUX MPS}

- Hernie ombilicale ou inguinale souvent récurrente ;

- Cou court ;

- Anomalies du squelette, gibbosité, pieds varus ou valgus ;

- infections ORL répétées ;

- faciès à traits grossiers ;

- limitation articulaire modérée ;

- foie et rate augmentés de volume ;

- valvulopathie mitrale ou aortique avec ou sans cardiomyopathie.

\section{Manifestations tardives :}

- Faciès aux traits grossiers avec macroglossie, gencives épaissies Dents espacées ;

- Main en griffe ;
- Cheveux épais en chaume ;

- Hydrocéphalie ;

- hépato-splénomégalie ;

- surdité

- opacification cornéenne, glaucome, dégénérescence rétinienne;

- apnées du sommeil ;

- Manifestations squelettiques dysostoses multiples ;

- Arthropathies : limitation articulaire sévère.

Les signes radiologiques ne sont pas pathognomoniques mais peuvent orienter de façon significative le diagnostic des MPS:

- selle turcique en oméga ;

- élargissement costal, des métas et des phalanges ;

- vertèbre cunéiforme, et vertèbres ovoïdes ;

- coxa valga, grignotage de l'acetabulum.

\section{LES DIFFÉRENTES MPS}

Il y a un continuum entre les différentes formes cliniques.

\section{La MPS I}

- Déficit en alpha L iduronidase

- Fréquence 1/100 000 à 1/280 000 naissances

- 3 types classiques: qui sont un continuum: on distingue selon un ordre de sévérité décroissante[5] :

$\checkmark$ Hurler (retard mental)

$\checkmark$ Hurler-Scheie (intelligence normale)

$\checkmark$ Scheie (intelligence normale)

La maladie de Hurler se distingue par l'atteinte cérébrale avec notamment un retard mental: l'espérance de vie est inférieure à 10 ans (figure 2).

La maladie de Hurler Scheie est caractérisée par l'absence d'atteinte du SNC, une atteinte somatique plus tardive que dans la maladie de Hurler et une espérance de vie plus longue (figure 3).

Enfin la maladie de Scheie est caractérisée par une espérance de vie beaucoup plus longue et une atteinte somatique très modérée.

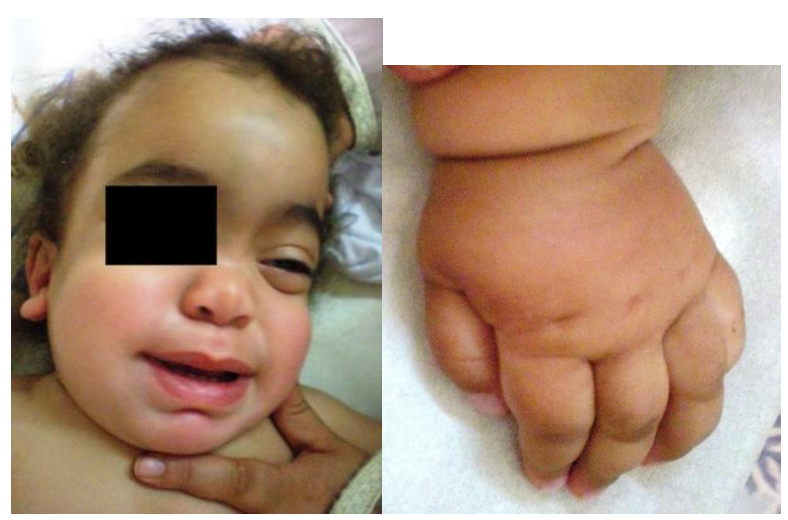

Figure 2 : Maladie de Hurler à l'âge de 3 ans, patiente décédée à l’âge de 6 ans par insuffisance cardiorespiratoire, noter le facies grossier et la main en griffe présents chez la plupart des patients atteints de MPS. 

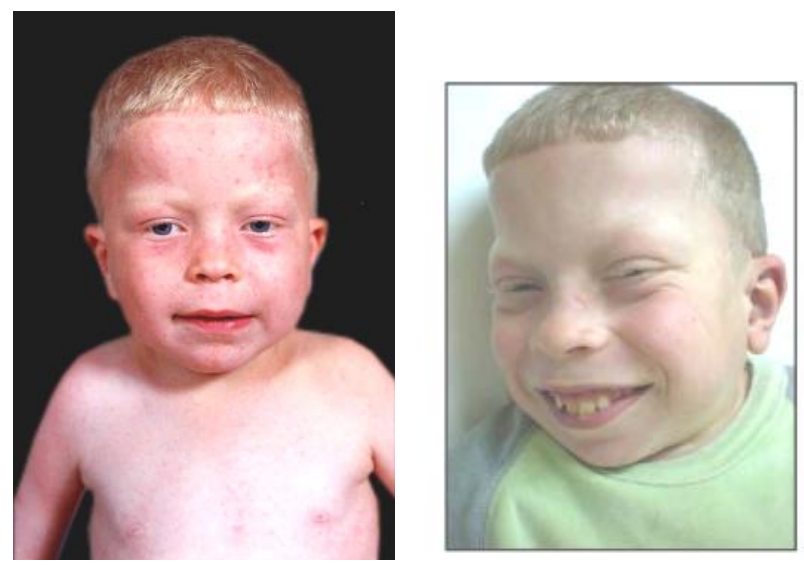

Figure 3 : Patient atteint de maladie de Hurler Scheie à l'âge de 6 ans et à l'âge de 11 ans. Noter l'épaississement progressif des traits après une période de 5 ans.

\section{La MPS II maladie de Hunter :}

Comme nous l'avons dit plus haut sa transmission se fait selon le mode lié à l'X.On retrouve à l'interrogatoire souvent des oncles maternels atteints.L'enzyme en cause est l'Iduronidase sulfatase nécessaire pour la dégradation de l'Héparane sulfate et le Dermatane sulfate. La fréquence est de $1 / 110000$ à $1 / 140000$ naissances [6]. Il existe 2 formes : une forme sévère avec retard mental et une forme atténuée avec une intelligence préservée.

$\checkmark$ La forme sévère a une espérance de vie limitée. Le décès survient généralement dans la 2 èmedécennie alors que le patient est dans un état grabataire (figure 4).

$\checkmark$ La forme atténuée peut permettre une espérance de vie normale (Figure 4).

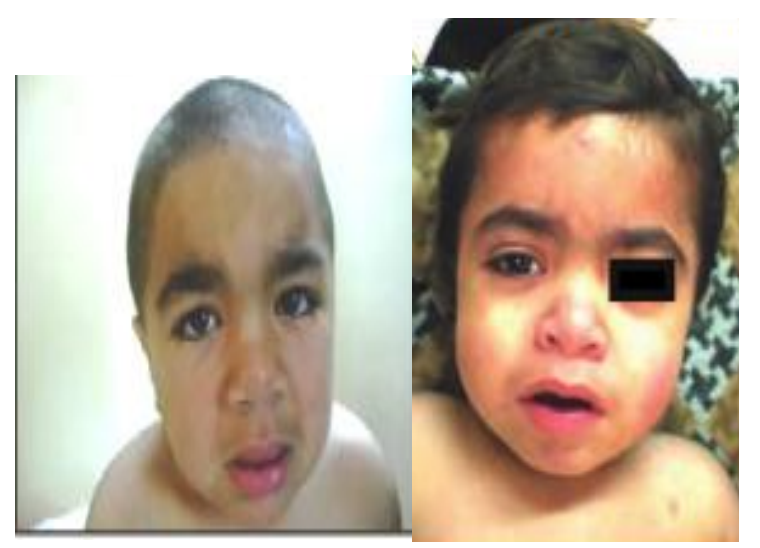

Figure 4: Maladie de Hunter. à gauche forme atténuée; à droiteforme sévère. Noter l'aspect grossier des traits chez les 2 patients, le traitement a été inefficace chez le 2 ème patient.

\section{La MPS III ou maladie de San Filippo :}

Il existe 4 différents types de la maladie selon le déficit enzymatique (figure 5) :

Type A : déficit en Héparane sulfatase ;

Type B: déficit en $\alpha$-N-acétyl glucosaminidase ;

Type C: déficit en Acétyl-CoA- $\alpha$-glucosamine-transférase ;

Type D : déficit en N-acétyl-glucosamine-6-sulfatase.

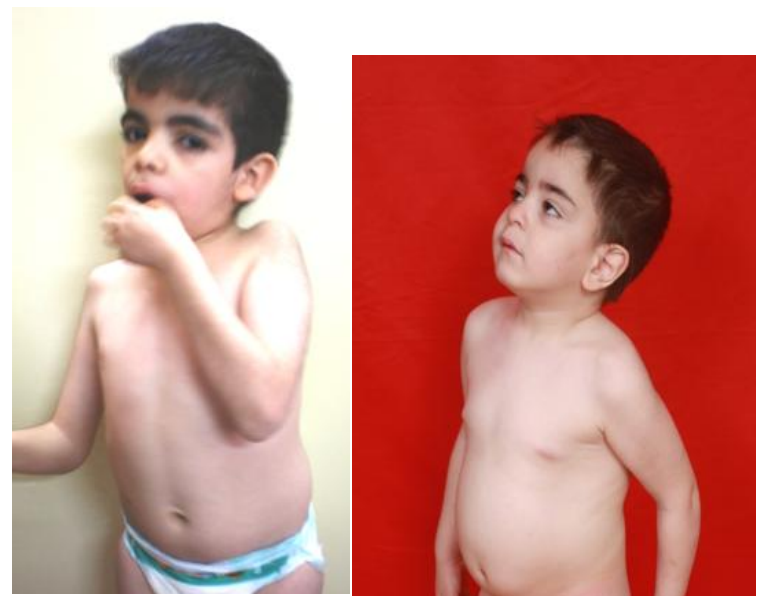

Figure 5 : à gauche MPS III type A; à droite MPS III type B. Noter l'attitude inappropriée chez les 2 enfants, les sourcils épais chez le patient de gauche et l'absence de traits grossiers chez le patient de droite.

On ne peut pas différencier les 4 types sur des signes cliniques mais le type $\mathrm{A}$ semble le plus sévère. Le début survient entre 2 et 6 ans par des troubles du comportement et un retard d'acquisitions cognitives. Les manifestations respiratoires avec infections et troubles du sommeil attirent peu l'attention mais ils sont souvent associés à des troubles du comportement avec hyperactivité et tendance à l'automutilation. L'apparition de traits grossiers, si caractéristiques des MPS, sont très discrets au début ou même absents. On ne les met en évidence que tardivement [5].Par ailleurs, il n'y a pas de retard staturo-pondéral au cours des premières années, au contraire on retrouve fréquemment une avance staturale jusqu'à l'âge de 10 ans puis une décélération importante survient. La dysostose est discrète.

Le malade perd peu à peu toutes les acquisitions psychomotrices, le contrôle sphinctérien, et sa mobilité.La dégradation intellectuelle est inéluctable aboutissant au décès à la fin de la 2 èmedécennie ou au début de l'âge adulte, souvent lors d'une infection respiratoire.

\section{La MPS IV ou maladie de Morquio :}

Deux enzymes sont à l'origine de la maladie : le IV A : dû à un déficit en $\mathrm{N}$-acétyl-galactosamine et le IV $\mathbf{B}$ à un déficit en $\beta$ galactosidase.L'aspect clinique est le même pour les 2 types, il est caractéristique avec un nanisme avec raccourcissement du tronc, des opacités cornéennes minimes, une dysplasie osseuse différente des autres MPS et une préservation de l'intelligence (Figure 6) [7].

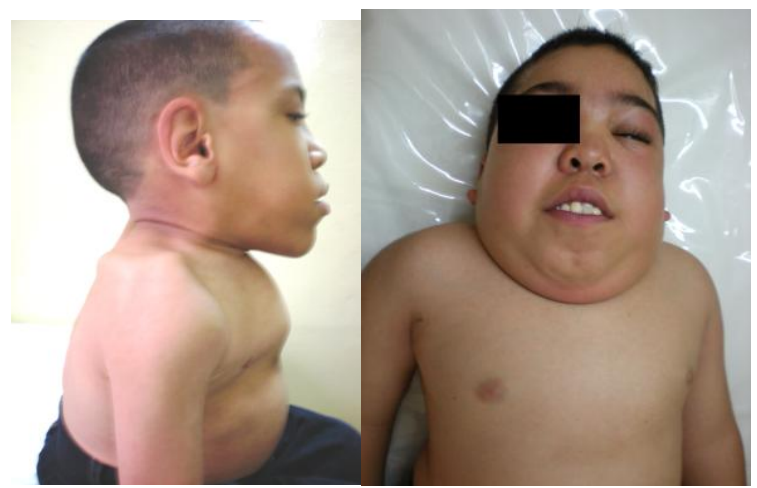

Figure 6 : MPS IV forme atténuée à gauche, forme grave à droite. Noter la protrusion sternale et l'hyperlaxité ligamentaire chez le patient de gauche, le patient de droite est décédé à l'âge de 14 ans des suites d'une insuffisance cardiorespiratoire. 
Les déformations osseuses sont différentes des autres MPS : genu valgum, hypoplasie de l'apophyse odontoïde à l'origine de l'instabilité de la colonne et de blessures de la moelle épinière.La radiographie de la colonne lombaire montre des spicules à l'extrémité des corps vertébraux.

\section{La MPS VI ou maladie de Maroteaux Lamy}

C'est la moins fréquente des MPS, 1/340 000 naissances environ[8]. L'enzyme déficient est l'arylsulfatase.Il y a également 2 formes: une forme sévère avec atteinte somatique plus marquée et une forme atténuée, dans les 2 cas l'intelligence est préservée (figure 7) [5].

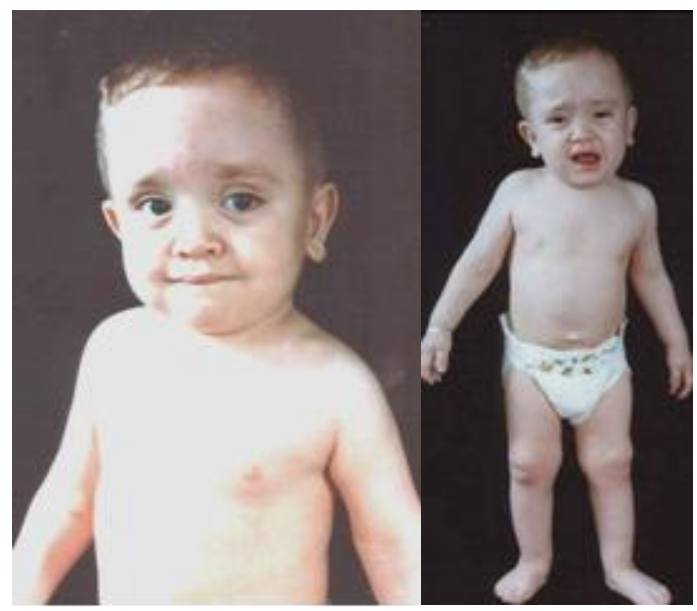

Figure 7 : MPS VI forme sévère: 2 frères atteints de la maladie actuellement grabataires en raison de coupures fréquentes du traitement

Les signes cliniques sont communs aux autres MPS avec une macrocrânie plus accentuée chez les patients atteints de MPS VI.

La MPS VII a les mêmes signes que la maladie de Hurler et ne peut être distinguée que par les études enzymatiques

La MPS IX est extrêmement rare et on n'a colligé que quelques patients dans le monde.

\section{PRISE EN CHARGE DES MPS}

L'Évaluation du degré de l'atteinte doit se faire à intervalles réguliers[9-11]:

1. Le syndrome d'obstruction des vois aériennes supérieures: épaississement de la langue et augmentation du volume des amygdales ;

2. Evaluation de la vision, de l'audition;

3. Recherche du syndrome du canal carpien (électromyogramme : les signes électriques précèdent les signes physiques) ;

4. Evaluation de la mobilité articulaire (goniomètre) des grosses articulations ;

5. Mesure du périmètre de marche durant 6 minutes $(6 \mathrm{mn}$ walk test) ;

6. Evaluation de l'atteinte cardiaque: échocardiographie, ECG : Il peut y avoir une atteinte des valvules mitrales et/ou aortiques, les artères coronaires sont le siège d'une obstruction parfois source d'ischémie.La myocardiopathie est la source principale de décès

7. L'atteinte de l'appareil respiratoire se manifeste par des déformations de la trachée à l'origine de difficultés majeures lors de l'intubation pour anesthésie et d'autre part l'atteinte interstitielle est à l'origine d'une hypo oxygénation.

8. la Recherche d'hydrocéphalie doit être systématique et peut nécessiter une dérivation ventriculo-péritonéale en cas d'hypertension intracrânienne. La compression spinale par épaississement de la dure-mère doit être surveillée par des examens successifs. Une laminectomie est indiquée si des signes cliniques apparaissent.

9. Nota Bene: L'anesthésie est un risque majeur pour les patients en raison de l'instabilité de l'atlas et de l'axis du fait de l'hypoplasie de l'apophyse odontoïde.

\section{Le diagnostic des MPS :}

Le diagnostic des MPS suspecté sur les signes cliniques, et est orienté par une analyse des GAG urinaires à la fois quantitative (augmentation et dans certaines formes très atténuées dans la plupart des cas mais pas toujours dans les MPS IV) et qualitative (mise en évidence de fraction anormales de dermatane sulfate, héparane sulfate et kératane sulfate permettant d'orienter le diagnostic (cf. tableau 2) $[12,13]$.

Tableau 2: Synopsis du diagnostic pour les MPS

MPS I : Héparane Sulfate et Dermatane Sulfate (HS et DS)

MPS II: HS, DS

MPS III: HS

MPS IV: KS (Kératane sulfate)

MPS VI: DS

MPS VII : CS ou CS + DS+ HS (CS= Chondroïtine sulfate)

MPS IX : Acide Hyaluronique

Le diagnostic de certitude fait appel à des méthodes enzymatiques à la génétique[14,15] :

- Recherche du déficit en cause sur des lymphocytes ou sur des fibroblastes.

- Actuellement le diagnostic enzymatique et éventuellement génétique peut se faire sur du papier buvard type "Guthrie» ou un papier filtre avec une bonne fiabilité[16].

- L'étude génétique peut mettre en évidence des génotypes susceptibles de traitement par greffe de moelle osseuse ou de par des cellules souches (comme dans la MPS I).

Le traitement symptomatique des MPS I-VII [17] fait appel à de nombreux spécialistes : Kinésithérapeute, cardiologue, et chirurgien cardiaque, ORL, Ophtalmologue, neurochirurgien, chirurgien, pneumologue.

Le traitement spécifique :Fait appel à 4 types de traitements :

La greffe de moelle osseuse, la greffe de cellules souches, l'enzyme recombinante et la thérapie génique.Un diagnostic précoce est impératif pour de meilleurs résultats car les séquelles sont généralement définitives.

L'enzyme de substitution est actuellement utilisée pour les MPS I, II, IV et VI. Elle est en cours d'évaluation pour les MPS III et VII.

MPS I : Aldurazyme (Laronidase) de Genzyme 100 $\mathrm{U} / \mathrm{Kg} /$ semaine ;

MPS II : Elaprase (Idursulfase) de Shire 0,5 mg/kg, et Hunterase (Idursulfase béta) de Green Cross à la même dose approuvée par la FDA et actuellement utilisée en Algérie et 
dans certains autres pays ;

MPS IV : Vimizim (Elosulfase alpha) utilisée aux USA et actuellement en Europe ;

MPS VI : Naglazyme (Galsulfase) par Biomarin à la dose de 1 $\mathrm{mg} / \mathrm{kg}$.

Greffe de moelle ou de cellules souches: Avantages: elle prévient l'atteinte cérébrale si utilisée tôt dans la MPS I avec génotypes: W402X et Q70X. Ces génotypes sont associés à une atteinte cérébrale si le traitement par la greffe ne se fait pas suffisamment tôt (avant 2 ans). Amélioration de la fonction cardiaque, respiratoire, articulaire, de l'audition, de la vision, diminution de l'hépatosplénomégalie.Inconvénients : risque de décès augmentés, risque de rejet.

Les enzymes recombinantes ou de substitution:avantages: amélioration de la mobilité articulaire, du périmètre de marche, de la fonction respiratoire, réduction de l'hépatosplénomégalie.Effets secondaires bénins : céphalées, fièvre, urticaire, effets secondaires graves: risque de réaction anaphylactique nécessitant un traitement d'urgence, troubles respiratoires.

La thérapie génique est actuellement utilisée dans certains centres (Bicêtre: Professeur Marc Tardieu : maladie de San Filippo B) : il s'agit de l'utilisation d'un virus génétiquement modifié et introduit dans le cerveau du patient. Le traitement n'est utilisé que chez des patients diagnostiqués par dépistage de la maladie dans la famille d'un patient atteint. Ce traitement doit être fait avant l'apparition des signes. Ce traitement est en cours d'essai clinique.

\section{CONCLUSION}

Les mucopolysaccharidoses sont des maladies rares mais compte tenu du contexte de consanguinité dans lequel nous vivons en Algérie, la fréquence est sûrement sous-estimée par manque d'information auprès des médecins généralistes. Un effort doit être fait à ce niveau pour détecter cette pathologie plus précocement et permettre un traitement rapide. Il est indispensable que les autorités fassent plus de facilités pour l'obtention des médicaments lorsque le diagnostic est établi et surtout qu'il n'y ait plus de rupture de stock comme c'est souvent le cas actuellement.

Par ailleurs, le traitement doit se faire au début par des équipes habituées à ce type de pathologie. Les malades doivent ensuite être transférés dans des hôpitaux au plus près de leur zone d'habitation pour éviter les déplacements fatigants pour les parents et les patients. Un consensus à l'échelle nationale est plus que souhaitable pour choisir la meilleure stratégie à prendre et également pour que les ressources financières soient utilisées le mieux possible, car le traitement de ces pathologies est extrêmement coûteux.
Déclaration d'intérêts : l'auteur ne déclare aucun conflit d'intérêt en rapport avec cet article.

\section{Messages clés}

$\checkmark \quad$ maladies lysosomales faciles à détecter pour la plupart d'entre elles par des signes communs: faciès grossier, cou court, gibbus, hépatosplénomégalie, retard staturopondéral, atteinte cérébrale avec régression psychomotrice chez certaines MPS.

$\checkmark$ Possibilité de traitement chez un grand nombre d'entre elles (greffe de moelle, enzymes de substitution, thérapie génique, et traitement des atteintes viscérales exemple adénoïdectomie, chirurgie cardiaque etc.)

\section{RÉFÉRENCES}

1. Baloghova J ; Chief Editor: Elston DM. Mucopolysaccharidoses Type I-VII : emedicine.medscape.com/article/1115193-overview

2. Mc Kusick VA. The Genetic mucopolysaccharidoses. Medicine 44:445-470, 1965

3. A Le Treut. Les glucides. Faculté de Rennes 1 Cours de Biochimie pour CPEM.

4. Andrieu N, Codogno P, Levade T. Les Lysosomes: Atlas des maladies lysosomales Vaincre les maladies lysosomales Généralités.

5. Neufeld ER, Muenzer J. The Mucopolysaccharidosis : in Scriver CR, Beaudet AL, Sly WS, The metabolic and Molecular Bases of Inherited Diseases 7 th ed. New York, Mc Graw-Hill, 1995, 3421-3451

6. Lowry RB, Applegarth DA, Toone JR, et al. Update on frequency of Mucopolysaccharide syndrome In British Columbia. Human genetic : 85:389, 1990

7. Montano AM, Tomatsu S, Gottesman GS, et al. International Morquio Registry: clinical manifestation and natural course of Morquio A disease. inherited Metab Dis. Apr 2007;30(2):165-14

8. Ben Turkia H, Tebib N, Azzouz $\mathrm{H}$, et al. Incidence des mucopolysaccharidoses en Tunisie. La Tunisie Médicale 2009; Vol 87(n¹1): 782-785

9. Muenzer J, Beck M, Eng CM, et al.Multidisciplary management of Hunter Disease. Pediatrics 2009; 124: e1228-39

10. Muenzer J, Wraith JE, Clarke LA. Mucolysaccharidosis: management and treatment guidelines. Pediatrics 2009; 123: 19-29

11. Valayanopoulos V, Nicely $\mathrm{H}$, Harmatz $\mathrm{P}$, et Al. Mucopolysaccharidosis VI. Orphanet Journal of Rare Diseases $2010 ; 5: 5$

12. Pennock CA. A review and selection of simple laboratory methods used for the study of glycosaminoglycan excretion and the diagnosis of the mucopolysaccharidoses. J Clin Pathol 29:111, 1976

13. Piraud M, Boyer S, Mathieu J, et al.Diagnosis of Mucopolysaccharidoses in a clinically selected population by urinary GAG analysis : a study of 2000 urine samples. Clinica Chimica Acta 1993, vol. 221(1)-171-81

14. Hall CW, Liebaers I, Di Natale P, et al. Enzymic diagnosis of the genetic mucopolysaccharide storage disorders. Methods Enzymol $50: 439,1978$

15. Kresse $\mathrm{H}$, Von Figura $\mathrm{K}$, Klein $\mathrm{U}$, et al. Enzymic diagnosis of the genetic mucopolysaccharide storage disorders. Methods Enzymol 83: 559, 1982

16. Gelb MH, Turecek F, Scott CR, et al. Direct multiplex assay of enzymes in dried blood spots by tandem spectrometry for the new born sreening of lysosomale storage disorders. J Inherit Metab Dis 2006; 29 : 397-404

17. Baloghova J, Schwartz RA, Zuzana Baranova, et al. Mucoplolysaccharidoses Types I-VII: treatment \& management. emedicine. Medscape.com/article/ 1115193-treatment

Cet article a été publié dans le « Batna Journal of Medical Sciences »BJMS, I'organe officiel de "I'association de la Recherche Pharmaceutique - Batna "

Le contenu de la Revue est ouvert « Open Access » et permet au lecteur de télécharger, d'utiliser le contenu dans un but personnel ou d'enseignement, sans demander l'autorisation de l'éditeur/auteur.

Pour plus d'informations, contacter BatnaJMS@gmail.com ou connectez-vous sur le site de la revue : www.batnajms.com 


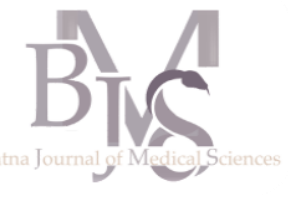

Faculté de Médecine, Université Hadj Lakhdar, Batna - Algérie

\section{Correspondance à :}

Ghania BELAALOUI

contact genetique@yahoo.fr

DOI :https://doi.org/10.48087/ B]MStf.2015.2111

Il s'agit d'un article en libre accès distribué selon les termes de la licence Creative Commons Attribution International License (CC BY 4.0), qui autorise une utilisation, une distribution et une reproduction sans restriction sur tout support ou format, à condition que l'auteur original et la revue soient dûment crédités.

\section{La maladie de Crohn : entre le déterminisme génétique et l'espoir épigénétique}

\section{Crohn's disease: between the genetic determinism and the epigenetic hope}

\section{Ghania Belaaloui}

\section{RÉSUMÉ}

La maladie de Crohn (MC) est une maladie inflammatoire chronique et multifactorielle. L'étiopathogénie de la maladie fait intervenir un état de dysbiose intestinale avec une altération de la perméabilité de la barrière épithéliale, ce qui entraîne une rupture de d'homéostasie intestinale et une réponse immunitaire aberrante. Ceci aboutit à un état inflammatoire chronique de l'intestin. La MC se développe chez des sujets génétiquement prédisposés, après exposition à certains facteurs environnementaux tels que le tabac et les facteurs modifiants le microbiote intestinal (comme le régime alimentaire et les antibiotiques). L'effet de l'environnement sur développement de la maladie semble se jouer via les modifications épigénétiques. L'étude de ces modifications a permis, non seulement une plus grande compréhension du mécanisme de la maladie, mais également l'ouverture de la voie d'utilisation de ces modifications épigénétiques pour développer des moyens de dépistage et de diagnostic. L'espoir d'une thérapie épigénétique est né récemment avec l'utilisation prometteuse des microRNA dans le traitement de colites provoquées chez des modèles expérimentaux murins.

Mots-clés : maladie de Crohn ; prédisposition génétique ; épigénétique ; environnement ; microRNA.

\begin{abstract}
Crohn's disease (CD) is a chronic, multifactorial inflammatory disease. Its pathogenesis involves an intestinal dysbiosis with an impaired permeability of the epithelial barrier. This induces a break of the intestinal homeostasis and an aberrant immune response. The final result is a chronic inflammatory condition of the bowel. CD develops in genetically predisposed subjects after exposure to some environmental factors such as tobacco and intestinal microbiota modifying factors (for instance diet and antibiotics). The effect of the environment on disease development seems to be conducted by epigenetic modifications. Studies of epigenetic modifications in CD contributed not only to a better conception of its mechanism, but opened also the way to the use of these epigenetic changes for the development of screening or diagnostic tools. The hope for an epigenetic therapy emerged recently with the promising use of microRNA in the treatment of induced colitis in murine experimental models.
\end{abstract}

Keywords: Crohn's disease; genetic predisposition; epigenetics; environment; microRNA.

\section{Pour citer l'article :}

Belaaloui G. La maladie de Crohn : entre le déterminisme génétique et l'espoir épigénétique. Batna J Med Sci 2015;2(1):45-49. https://doi.org/10.48087/ BJMStf.2015.2111

\section{داء الكرون: بين التحديد الوراثي وأمل علم التخلق الوراثي}

الكلمات المفتاحية: داء الكرون، الاستعداد الوراثي، علم التخلق الوراثي (أبيجيناتيكس)، البيئة، الحمض الريبونووي الدقيق (ميكرورنا) 


\section{INTRODUCTION}

La maladie de Crohn (MC) est une maladie inflammatoire chronique de l'intestin (MICI) qui touche préférentiellement l'iléon terminal, bien qu'elle puisse toucher tout le reste du tractus digestif. Elle se présente fréquemment par une diarrhée chronique avec des douleurs abdominales et une perte de poids. Son diagnostic repose sur un faisceau d'arguments cliniques, biologiques, radiologiques, endoscopiques et histologiques [1], et son évolution est parsemée de complications et finit souvent par avoir de fortes répercussions sur la qualité de vie des patients.

La MC touche typiquement les sujets des deux sexes âgés entre 20 et 40 ans, mais toutes les tranches d'âges peuvent se voir. Son incidence est influencée, en partie, par l'appartenance ethnique et la localisation géographique: elle suit classiquement un gradient décroissant Nord-Sud [2]. Bien que relativement rare en Afrique, une augmentation de son incidence a été signalée ces dernières années [3, 4]. En Algérie, nous ne retrouvons pas de données épidémiologiques publiées mais les statistiques hospitalières dénotent également l'augmentation de son incidence ces dernières années [5].

\section{ETIOPATHOGÉNIE DE LA MALADIE DE CROHN}

La MC est une maladie multifactorielle où des facteurs génétiques s'entremêlent avec des facteurs environnementaux dont la connaissance est encore loin d'arriver à expliquer totalement la pathogénie. Cependant, les données actuelles permettent de penser que le point de départ de la MC est une réponse immunitaire aberrante contre le microbiote intestinal (flore intestinale) chez des personnes qui sont génétiquement prédisposées. En d'autre terme, il existerait une rupture de l'état d'homéostasie intestinale chez ces personnes (Figure 1). L'homéostasie intestinale peut être définit, quant à elle, comme étant le monitoring et la régulation de l'interaction du microbiote avec la surface épithéliale intestinale par le système immunitaire inné et adaptatif de l'intestin [6].

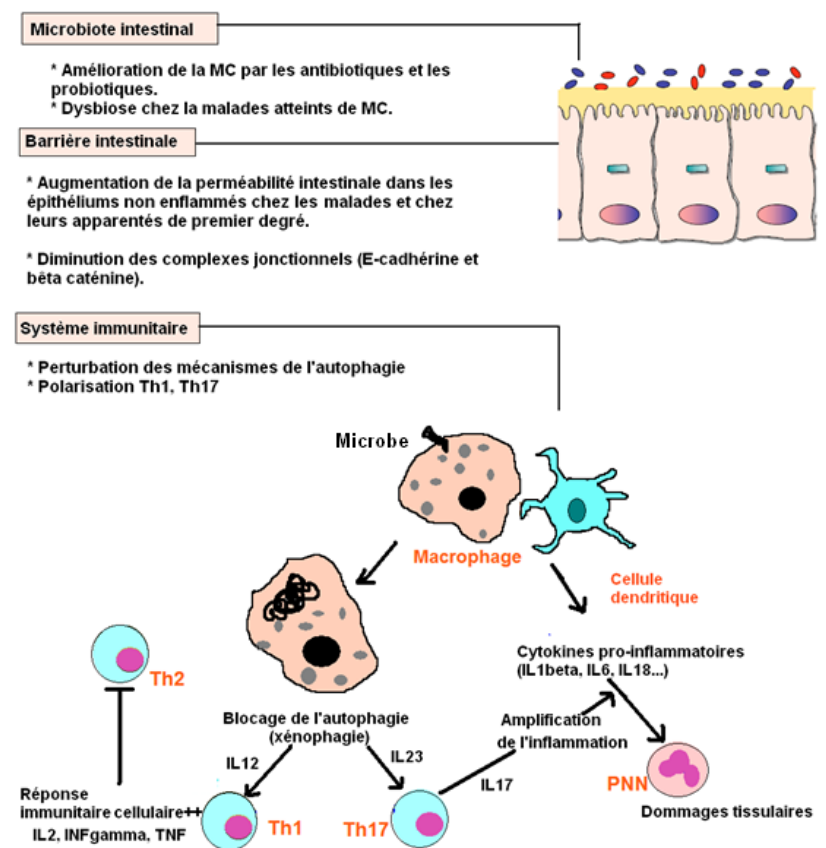

Fig 1 : Etiopathogénie de la MC. La MC résulte d'une rupture de l'état d'homéostasie intestinale impliquant le microbiote, la barrière intestinale et le système immunitaire inné et adaptatif. (MC : maladie de Crohn. PNN : polynucléiares neutrophiles).

\section{L'implication du microbiote}

La part du microbiote dans pathogénèse de la MC est démontrée, indirectement, par la découverte d'une dysbiose (modification du microbiote intestinal) chez les malades [7, 8], l'amélioration de l'état de ces derniers après antibiothérapie [9] et après administration de certains probiotiques $[10,11]$.

\section{Le rôle de la barrière intestinale}

L'intégrité de la barrière épithéliale intestinale est primordiale pour le maintien de l'homéostasie intestinale. Elle est assurée essentiellement par le mucus et par les jonctions intercellulaires qui sont dynamiquement régulées par des cytokines [12]. Dans l'épithélium iléal non enflammé des patients atteints d'une MC [13] et celui de leurs parents de premier de degré [14], une augmentation de la perméabilité intestinale aux macromolécules a été observée.

\section{Le dérèglement immunitaire}

A l'état normal d'homéostasie, il existe une inflammation physiologique de la muqueuse intestinale [15], où les cellules épithéliales et les cellules de Paneth, grâce à leurs récepteur de reconnaissance des pathogènes PRR (Pathogen recognition receptors), reconnaissent les PAMPs (Pathogen Associated Molecular Pattern) exprimés par les microbes, d'où la production de zinc, de lysozyme et d'autres molécules antimicrobiennes comme les défensines [12]. De plus, l'autophagie, qui est un processus de dégradation des agrégats protéiques et des organelles endommagés, est un autre mécanisme de défense innée qui intervient à l'état physiologique, elle consiste en l'élimination des pathogènes ayant parvenu à entrer en intracellulaire et elle est alors appelée xénophagie [16]. Un défaut de l'autophagie a été observé dans la MC et il a suscité d'énormes intérêts ces dernières années. Ce défaut pourrait conduire à la multiplication intracellulaire du microbe et une inflammation chronique et non contrôlée dans l'intestin [1719]. Ainsi, la sécrétion de cytokines pro-inflammatoires, qui en résulte, favorise l'activation de plusieurs cellules dont les neutrophiles qui vont engendrer des dommages tissulaires au niveau de l'intestin, et les lymphocytes $T$ qui sont polarisés vers les voies Th1 et Th17 au détriment de la voie Th2 [20].

\section{La prédisposition génétique}

Durant les 25 dernières années, la prédisposition génétique aux MICI a été largement explorée, à tel point qu'un consortium international sur la génétique des MICI (Inflammatory Bowel Disease Genetics Consortium (IIBDGC)) a été créé [21]. Les études d'association à large échelle «GWAS» (Genome-Wide Association Studies) ont permis ainsi d'associer les polymorphismes génétiques de plusieurs loci et gènes à la susceptibilité aux MICI. Une dernière métaanalyse révèle l'implication de 163 loci dans le risque des MICI, dont 30 sont spécifiques à la MC [22]. La plupart de ces gènes sont impliqués dans la réponse immunitaire et dans la fonction de la barrière épithéliale intestinale, à l'exemple du :

- Gène NOD2 (Nucleotide-binding Oligomerisation Domaincontaining 2) qui est un gène majeur de susceptibilité à la MC qui code pour un PRR, et des gènes ATG16L1 (autophagy-related 16 like-1) et IRGM, qui sont tous impliqués dans le processus d'autophagie.

- Gène du récepteur de l'IL23 (IL23R) et d'autres gènes impliqués dans la voie de différenciation Th17 (IL12B, JAK2, STAT3, CCR6 and TNFSF15) 
- Gène de l'IL-10.

- Gènes régulateurs des fonctions immunes tel que: PTPN22, CARD9, IL1R2, REL, SMAD3, et PRDM1.12.

- Gènes contrôlant la perméabilité intestinale: (EP4, OCTN1/2, mdr-1)

Beaucoup de ces loci sont impliqués également dans d'autres maladies immunologiques notamment la spondylarthrite ankylosante et le psoriasis [23].

\section{Les facteurs environnementaux}

L'augmentation de l'incidence de la MC dans certains pays asiatiques dont le mode de vie s'est occidentalisé ainsi que chez des ethnies originaires du Sud après leur installation dans des pays nordiques, témoigne d'un virage épidémiologique important qui démontre l'effet de l'environnement sur le risque de développer la MC [2]. De plus, les études génétiques avaient déjà montré un important taux de discordance de survenue de la maladie entre les jumeaux monozygotes [24]. Parmi les facteurs de risque environnementaux identifiés, on retrouve le tabagisme, le régime alimentaire et le mode de vie ainsi que le facteur psychique et la perturbation $\mathrm{du}$ microbiote par les antibiotiques [2].

\section{QUAND LA GÉNÉTIQUE NOUS MÈNE VERS L'ÉPIGÉNÉTIQUE}

Ce sont les résultats des GWAS qui ont ouvert la piste de l'épigénétique dans la $\mathrm{MC}$ en découvrant des polymorphismes génétiques prédisposant à la MC qui sont localisés sur les gènes des enzymes de régulation épigénétique comme la DNA methyltransferase (DNMT) 3a et la DNMT3b [22]. C'est ainsi que l'étude des modifications épigénétiques (ME) dans la MC n'a pas cessé de croitre [25] et il semble alors que ce sont ces modifications qui font le lien entre la susceptibilité génétique à la maladie et l'environnement (Figure 2).

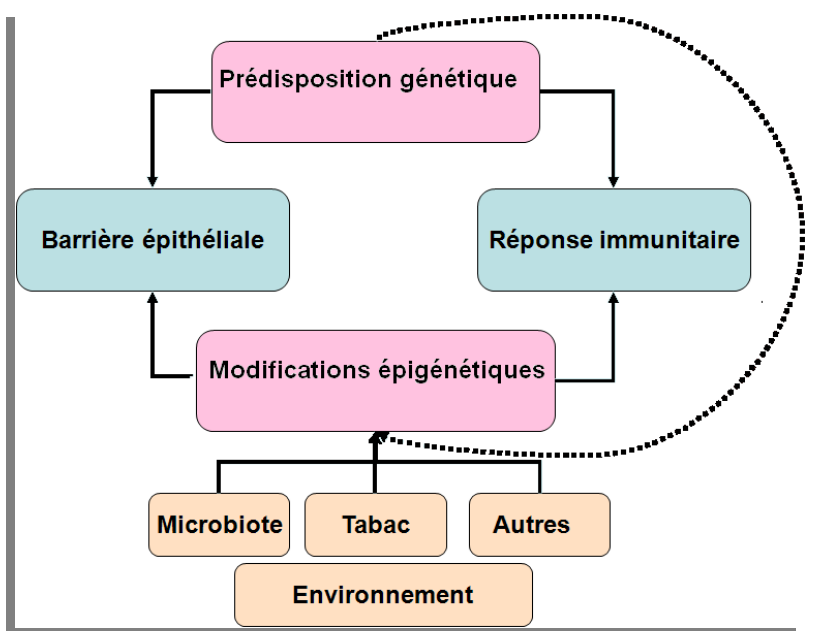

Figure 2 : Influences génétiques et épigénétiques sur le risque de la MC. Des variants génétiques et des modifications épigénétiques touchant des régions du génome qui sont impliquées dans la réponse immunitaire et dans l'intégrité de la barrière épithéliale intestinale, ont été identifiés. Les modifications épigénétiques semblent être le résultat de l'effet des facteurs environnementaux. Les modifications épigénétiques obéissent elles même à un déterminisme génétique.

\section{Les modifications épigénétiques dans la MC}

L'épigénétique consiste en l'ensemble des modifications possibles de l'expression des gènes (activation ou inactivation) sans changements des séquences nucléotidiques de ces derniers mais en en apportant des modifications biochimiques (méthylations, acétylations, phosphorylations...) à l'ADN et/ou aux histones, ou bien en interférant avec l'expression des gènes via des ARN non codants (ncRNA, non coding $R N A$ ), ces derniers peuvent être longs (long ncRNAs) ou petits (small ncRNAs). Les small ncRNAs comportent, entre autres, des siRNAs (short interfering RNAs) et des miRNAs (microRNAs)). Les ME sont caractérisées par le fait qu'elles soient largement transmissibles après les mitoses mais elles sont également réversibles. L'épigénétique a été impliquée dans plusieurs phénomènes physiologiques telle que la spécialisation tissulaire lors de l'embryogénèse, l'inactivation de l'un des chromosomes X chez la femme ou bien l'empreinte parentale sur certains gènes (imprinting)[26, 27].

Les ME sont influencées par plusieurs facteurs : génétiques (les enzymes qui font ses modifications étant codées par des gènes), environnementaux, et aussi stochastiques (facteurs non encore déterminés cachés sous ce terme qui signifie 'aléatoires') [28, 29]. Les influences de l'environnement sur les ME commencent dès la vie fœtale et continuent tout le long de la vie de l'individu [29].

Des ME 'anormales' ont été observées dans nombre de pathologies chroniques tel que le cancer, le diabète de type 2 , la maladie d'Alzheimer...etc. Dans le cas de la MC, les études épigénétiques ont montré qu'une cinquantaine de gènes des leucocytes circulants sont méthylés de manière différentielle entre les malades et les témoins sains, ce sont majoritairement des gènes impliqués sans la réponse immunitaire: IL12/IL23, NOD2, MAPK, DOK2, Tap1, TNF, ULK1...[30]. De plus, une importante acétylation de l'histone H4 a été observée dans les cellules des épithéliums enflammés des malades par rapport aux épithéliums sains [31]. L'acétylation des histones intervient dans la régulation de plusieurs gènes impliqués dans l'inflammation tels que NOD2 et TLR4 $[32,33]$. Enfin, des variations des quantités de certains miRNA (miR21, miR155, miR196...) dans les cellules épithéliales ou dans les leucocytes circulants des malades ont été observées après comparaison avec des sujets sains [25].

\section{L'espoir épigénétique dans la MC}

A l'instar de l'engouement qu'avait suscité l'épigénétique en oncologie, à la fois comme un possible moyen de dépistage, de diagnostic, de prévention ou de thérapie, on retrouve le même enthousiasme chez les scientifiques dans le cas de la MC. Ainsi, des études ont montré que la variation de la quantité de certains miRNA entre la muqueuse colique intacte des patients atteints d'une MC et celle des patients atteints de rectocolite ulcérative, pourrait constituer un moyen de diagnostic différentiel entre ces deux entités histopathologiques [34, 35]. Et depuis la découverte de miRNA circulants dans le sérum sous forme empaquetée dans des vésicules très stables et protégées contre la dégradation, l'espoir est de les utiliser comme biomarqueurs pour le diagnostic de la maladie [35].

La variation de la méthylation de certains gènes a même été proposée comme moyen de prédiction de développement d'un cancer chez les malades souffrant d'une MC, ainsi la méthylation des gènes SLIT2 et TMEFF2 est plus fréquente 
dans la muqueuse des patients à haut risque par rapport à ceux qui sont à faible risque de dysplasie ou de cancer [37].

Cependant, la question de la possible survenue des ME suite à la MC ou aux traitements reçu, et non pas l'inverse, se pose toujours. Ainsi, et afin de mieux positionner les ME dans de la $\mathrm{MC}$, le projet IBD-character (Inflammatory Bowel Disease CHARACTERization) regroupant dix partenaires européens académiques et industriels a été créé en 2012 [38]. Il vise à générer la plus grande collection d'échantillons chez des patients récemment diagnostiqués pour des MICI et n'ayant pas encore reçu de traitement. Une analyse et une caractérisation approfondies de l'état de méthylation de l'ADN, celui du transcriptome, des marqueurs protéiques, du microbiote intestinal, du génotype des patients pour les loci de susceptibilité connus, ainsi que des données cliniques des malades, permettra d'avoir un aperçu moléculaire des MICI, dont la MC, au début de leur manifestation [38].

Enfin, il ne faut pas oublier de dire que le caractère réversible des modifications épigénétiques a fomenté lui aussi d'énormes intérêts dans le contexte de la MC, à l'instar d'autres pathologies. L'espoir a été récemment donné par des modèles murins où l'utilisation de miRNA inhibant la voie Th17 (miR-155, miR-210) [39, 40] ou le trafic des leucocytes (miR-141) [41] a permis d'éviter le développement de colites ulcéreuses induites.

Déclaration d'intérêts : l'auteur ne déclare aucun conflit d'intérêt en rapport avec cet article.

\section{RÉFÉRENCES}

1. Kalla, R., N.T. Ventham, J. Satsangil.D. Arnott. Crohn's disease. Bmj 2014; 349: g6670.

2. Ananthakrishnan, A.N. Epidemiology and risk factors for IBD. Nat Rev Gastroenterol Hepatol 2015; 12(4): 205-217.

3. Archampong, T.N.K.N. Nkrumah. Inflammatory bowel disease in Accra: what new trends. West Afr J Med 2013; 32(1): 40-44.

4. Ukwenya, A.Y., A. Ahmed, V.I. OdigieA. Mohammed. Inflammatory bowel disease in Nigerians: still a rare diagnosis? Ann Afr Med 2011; 10(2): 175-179.

5. Berkane, S., La maladie de Crohn, pathologie, symptômes, diagnostics et moyens préventifs, in Santé Mag. 2012. p. 46-47.

6. Duerkop, B.A., S. VaishnavaL.V. Hooper. Immune responses to the microbiota at the intestinal mucosal surface. Immunity 2009; 31(3): 368-376.

7. Ott, S.J., M. Musfeldt, D.F. Wenderoth, J. Hampe, O. Brant, et al. Reduction in diversity of the colonic mucosa associated bacterial microflora in patients with active inflammatory bowel disease. Gut 2004; 53(5): 685-693.

8. Urcelay, E., J.L. Mendoza, M.C. Martin, A. Mas, A. Martinez, et al. MDR1 gene: susceptibility in Spanish Crohn's disease and ulcerative colitis patients. Inflamm Bowel Dis 2006; 12(1): 33-37.

9. Wang, S.L., Z.R. WangC.Q. Yang. Meta-analysis of broad-spectrum antibiotic therapy in patients with active inflammatory bowel disease. Exp Ther Med 2012; 4(6): 1051-1056.

10. Celiberto, L.S., R. Bedani, E.A. RossiD.C. Cavallini. Probiotics: The Scientific Evidence in the Context of Inflammatory Bowel Disease. Crit Rev Food Sci Nutr 2015: 0.

11. Wasilewski, A., M. Zielinska, M. StorrJ. Fichna. Beneficial Effects of Probiotics, Prebiotics, Synbiotics, and Psychobiotics in Inflammatory Bowel Disease. Inflamm Bowel Dis 2015.
12. Garrett, W.S., J.I. GordonL.H. Glimcher. Homeostasis and inflammation in the intestine. Cell 2010; 140(6): 859-870.

13. Soderholm, J.D., G. Olaison, K.H. Peterson, L.E. Franzen, T. Lindmark, et al. Augmented increase in tight junction permeability by luminal stimuli in the non-inflamed ileum of Crohn's disease. Gut 2002; 50(3): 307-313.

14. Irvine, E.J.J.K. Marshall. Increased intestinal permeability precedes the onset of Crohn's disease in a subject with familial risk. Gastroenterology 2000; 119(6): 1740-1744.

15. Chassaing, B.A. Darfeuille-Michaud. The commensal microbiota and enteropathogens in the pathogenesis of inflammatory bowel diseases. Gastroenterology 2011; 140(6): 1720-1728.

16. Deretic, V.B. Levine. Autophagy, immunity, and microbial adaptations. Cell Host Microbe 2009; 5(6): 527-549.

17. Henderson, P.C. Stevens. The role of autophagy in Crohn's disease. Cells 2012; 1(3): 492-519.

18. Muzes, G., Z. TulassayF. Sipos. Interplay of autophagy and innate immunity in Crohn's disease: a key immunobiologic feature. World J Gastroenterol 2013; 19(28): 4447-4454.

19. Stappenbeck, T.S., J.D. Rioux, A. Mizoguchi, T. Saitoh, A. Huett, et al. Crohn disease: a current perspective on genetics, autophagy and immunity. Autophagy 2011; 7(4): 355-374.

20. Xavier, R.J.D.K. Podolsky. Unravelling the pathogenesis of inflammatory bowel disease. Nature 2007; 448(7152): 427-434.

21. IIBDGC. http://www.ibdgenetics.org/. Accessed on June 7th 2015.

22. Jostins, L., S. Ripke, R.K. Weersma, R.H. Duerr, D.P. McGovern, et al. Host-microbe interactions have shaped the genetic architecture of inflammatory bowel disease. Nature 2012; 491(7422): 119-124.

23. Lees, C.W., J.C. Barrett, M. ParkesJ. Satsangi. New IBD genetics: common pathways with other diseases. Gut 2011; 60(12): 17391753.

24. Tysk, C., E. Lindberg, G. JarnerotB. Floderus-Myrhed. Ulcerative colitis and Crohn's disease in an unselected population of monozygotic and dizygotic twins. A study of heritability and the influence of smoking. Gut 1988; 29(7): 990-996.

25. Ventham, N.T., N.A. Kennedy, E.R. NimmoJ. Satsangi. Beyond gene discovery in inflammatory bowel disease: the emerging role of epigenetics. Gastroenterology 2013; 145(2): 293-308.

26. Qiu, J. Epigenetics: unfinished symphony. Nature 2006; 441(7090) 143-145.

27. Dupont, C., D.R. ArmantC.A. Brenner. Epigenetics: definition, mechanisms and clinical perspective. Semin Reprod Med 2009; 27(5): 351-357.

28. Aguilera, O., A.F. Fernandez, A. MunozM.F. Fraga. Epigenetics and environment: a complex relationship. J Appl Physiol (1985) 2010; 109(1): 243-251.

29. Fraga, M.F. Genetic and epigenetic regulation of aging. Curr Opin Immunol 2009; 21(4): 446-453.

30. Nimmo, E.R., J.G. Prendergast, M.C. Aldhous, N.A. Kennedy, P. Henderson, et al. Genome-wide methylation profiling in Crohn's disease identifies altered epigenetic regulation of key host defense mechanisms including the Th17 pathway. Inflamm Bowel Dis 2012; 18(5): 889-899.

31. Tsaprouni, L.G., K. Ito, J.J. Powell, I.M. AdcockN. Punchard. Differential patterns of histone acetylation in inflammatory bowe diseases. J Inflamm (Lond) 2011; 8(1): 1.

32. Leung, C.H., W. Lam, D.L. Ma, E.A. GullenY.C. Cheng. Butyrate mediates nucleotide-binding and oligomerisation domain (NOD) 2 dependent mucosal immune responses against peptidoglycan. Eur J Immunol 2009; 39(12): 3529-3537. 
33. Takahashi, K., Y. Sugi, A. HosonoS. Kaminogawa. Epigenetic regulation of TLR4 gene expression in intestinal epithelial cells for the maintenance of intestinal homeostasis. J Immunol 2009; 183(10): 6522-6529.

34. Wu, F., M. Zikusoka, A. Trindade, T. Dassopoulos, M.L. Harris, et al. MicroRNAs are differentially expressed in ulcerative colitis and alter expression of macrophage inflammatory peptide-2 alpha. Gastroenterology 2008; 135(5): 1624-1635 e1624.

35. Fasseu, M., X. Treton, C. Guichard, E. Pedruzzi, D. Cazals-Hatem, et al. Identification of restricted subsets of mature microRNA abnormally expressed in inactive colonic mucosa of patients with inflammatory bowel disease. PLoS One 2010; 5(10).

36. Chen, X., Y. Ba, L. Ma, X. Cai, Y. Yin, et al. Characterization of microRNAs in serum: a novel class of biomarkers for diagnosis of cancer and other diseases. Cell Res 2008; 18(10): 997-1006.
37. Azuara, D., F. Rodriguez-Moranta, J. de Oca, X. Sanjuan, J. Guardiola, et al. Novel methylation panel for the early detection of neoplasia in high-risk ulcerative colitis and Crohn's colitis patients. Inflamm Bowel Dis 2013; 19(1): 165-173.

38. IBDcharacter. http://www.ibdcharacter.eu/. Accessed on June 7th 2015.

39. Singh, U.P., A.E. Murphy, R.T. Enos, H.A. Shamran, N.P. Singh, et al. miR-155 deficiency protects mice from experimental colitis by reducing $T$ helper type 1/type 17 responses. Immunology 2014; 143(3): 478-489.

40. Wang, H., H. Flach, M. Onizawa, L. Wei, M.T. McManus, et al. Negative regulation of Hif1a expression and TH17 differentiation by the hypoxia-regulated microRNA miR-210. Nat Immunol 2014; 15(4): 393-401.

41. Huang, Z., T. Shi, Q. Zhou, S. Shi, R. Zhao, et al. miR-141 Regulates colonic leukocytic trafficking by targeting CXCL12beta during murine colitis and human Crohn's disease. Gut 2014; 63(8): 12471257.

Cet article a été publié dans le "Batna Journal of Medical Sciences » BJMS, l'organe officiel de "I'association de la Recherche Pharmaceutique - Batna "

Le contenu de la Revue est ouvert « Open Access » et permet au lecteur de télécharger, d'utiliser le contenu dans un but personnel ou d'enseignement, sans demander l'autorisation de l'éditeur/auteur.

Avantages à publier dans BJMS :

- Open access : une fois publié, votre article est disponible gratuitement au téléchargement

- Soumission gratuite : pas de frais de soumission, contrairement à la plupart des revues « Open Access »

- Possibilité de publier dans 3 langues : français, anglais, arabe

- Qualité de la relecture : des relecteurs/reviewers indépendants géographiquement, respectant l'anonymat, pour garantir la neutralité et la qualité des manuscrits.

Pour plus d'informations, contacter BatnaJMS@gmail.com

ou connectez-vous sur le site de la revue : www.batnajms.com 


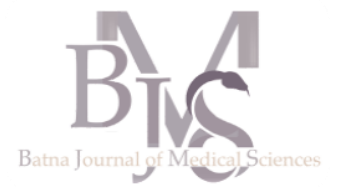

Faculté de Médecine, Université Abou Bekr Belkaid, Tlemcen Algérie

\section{Correspondance à :}

Mahfoud CHERIF BENMOUSSA

cbenmaf@gmail.com

DOI :https://doi.org/10.48087/ BJMStf.2015.2112

Il s'agit d'un article en libre accès distribué selon les termes de la licence Creative Commons Attribution International License (CC BY 4.0), qui autorise une utilisation, une distribution et une reproduction sans restriction sur tout support ou format, à condition que l'auteur original et la revue soient dûment crédités.

Pour citer l'article :

Cherfi Benmoussa M, Grari R, Sari Hamidou R, et al. La génétique et l'épigénétique au service des maladies rénales chroniques. Batna J Med Sci 2015;2(1):50-55. https://doi.org/10.48087/ BJMStf.2015.2112

\title{
La génétique et l'épigénétique au service des maladies rénales
}

\section{Genetics and epigenetics at the service of kidney diseases}

\author{
Mahfoud Cherif Benmoussa, Riad Grari, Rawda Sari Hamidou, Mustapha Benmansour
}

\section{RÉSUMÉ}

La maladie rénale chronique augmente le risque de morbi-mortalité. Le diagnostic est aisé mais rétrospectif, les facteurs de risques sont codifiés et ainsi que les principales cibles thérapeutiques. L'épigénome, interface entre le génome et le phénotype, semble attiser l'attention de la médecine moderne pour établir de nouvelles méthodes diagnostiques précoces et adopter une prise en charge personnalisée pour une meilleure efficience.

Mots-clés : maladie rénale chronique, génétique, épigénétique, facteur de risque, cardiovasculaire.

\begin{abstract}
The chronic kidney disease increases the risk of morbi-mortality. Diagnosis is easy but made retrospectively; risk factors are codified as well as main therapeutic targets. The epigenome, interface between the genome and the phenotype, seems to stir the attention of the modern medicine, in order to establish new early diagnostic methods and adopt a personalized management for a better efficacy.
\end{abstract}

Keywords: chronic kidney disease, genetics, epigenetics, risk factor, cardiovascular.

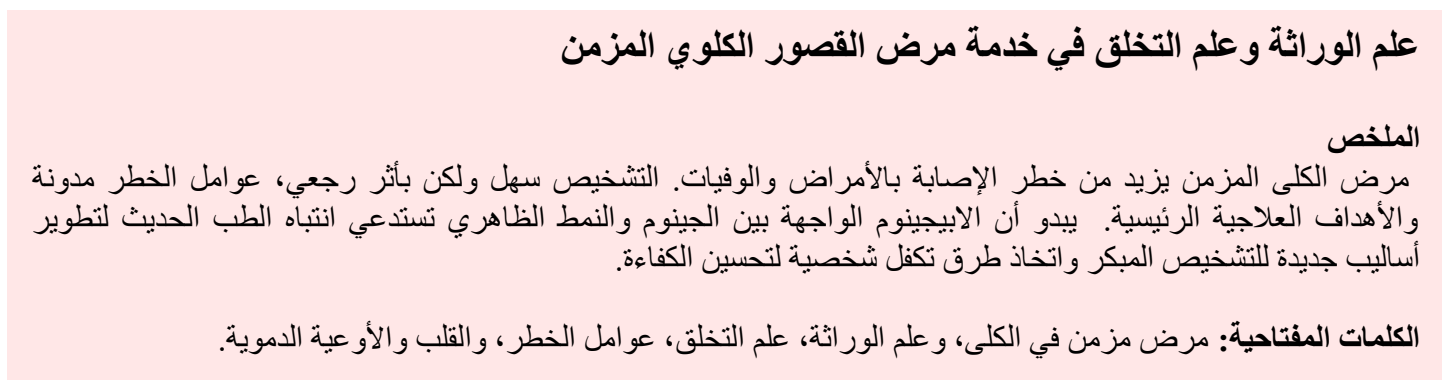

\section{INTRODUCTION}

La maladie rénale chronique constitue un problème de santé publique aussi bien dans les pays développés mais aussi dans les pays en voie de développement. L'atteinte rénale augmente le risque d'évènement cardiovasculaire [1], elle augmente aussi le risque de décès toute cause confondue [2].

La réduction néphronique se traduit par un remaniement de la structure histologique des néphrons soumis à des agressions au cours des pathologies systémiques dominées par le diabète et l'hypertension artérielle. Au cours des dernières décennies, les travaux se sont intéressés à établir le rôle de plusieurs facteurs dits facteurs de risques non héritables de dégradation de la fonction rénale pour en reconnaitre la micro-albuminurie, la protéinurie l'hypertension artérielle, l'hyperglycémie, la dyslipidémie, les dépôts minéraux (calcium, urée etc.), les drogues néphrotoxiques et les maladies inflammatoires.

Des individus issus d'une même population, soumis aux mêmes facteurs de risques traités par des protocoles similaires peuvent évoluer différemment. De ce principe il est nécessaire d'intégrer une approche personnalisée via la génétique.

Les raisonnements physiopathologiques semblent ne plus se limiter aux facteurs de risques connus à ce jour comme la microalbuminurie, la protéinurie, la pression artérielle, la dyslipidémie avec un impact d'exposition environnemental [3]. La perte néphronique qu'elle soit non spécifique, glomérulaire ou tubulaire implique une activation de plusieurs voies comme les cytokines, chémokines et facteurs de croissance déséquilibrant la balance formation dégradation, avec accumulation des produits inflammatoires et fibroses [4,5].

L'évaluation biologique de la fonction rénale utilisée de nos jours reste assez sommaire ; elle mesure le débit de filtration du glomérule [6,7]. La «créatinine » reste toujours la molécule de référence pour l'évaluation de la fonction rénale. Les méthodes de calcul ont évolué depuis la formule de cockroft et gault à la méthode de MDRD «modified diet renal desease »; les méthodes de dosages biologiques deviennent plus spécifiques 
passant du dosage par les réactions chimiques à l'acide picrique au dosage enzymatique. Les marqueurs tels que la cystatine C, KIM1, IL18 sont prometteurs sans rivaliser avec la créatinine et peu applicables en pratique clinique quotidienne. Pour conclure, ces évaluations ne sont que le reflet de lésions histologiques installées et un organe déjà atteint.

L'histologie accessible pour cet organe est un examen incontournable dans la pratique du néphrologue dans plusieurs contextes pathologiques. Le geste est invasif et ses indications sont bien encadrées. La génétique est incontournable dans la prise en charge des maladies rénales héréditaires, l'appliquer pour les diagnostics précoces dans des néphropathies plus répandues semble être accessible.

\section{QU'EST CE QUE LA GÉNÉTIQUE ET L'ÉPIGÉNÉTIQUE}

Gregor Mendel à la fin du XIXe siècle consigne ses observations sur des croisements entre variétés de petits pois. En 1953, c'est au tour de James Watson et Francis Crick d'apporter leurs contributions à la génétique en découvrant la structure en double hélice de l'ADN. Sydney Brenner, François Jacob et Matthew Meselson mettront ensuite en évidence l'existence de molécules, les ARN messagers, lien entre l'ADN et les protéines. Cependant, alors que toutes nos cellules sont porteuses de la même information génétique, il existe une centaine de types cellulaires, aux fonctions et propriétés différentes : cela prouve que la séquence du génome ne peut pas tout expliquer. Il apparaît évident qu'il existe un phénomène qui dans l'expression des gènes permet la diversification des potentialités génétiques au sein des différents types de cellules et est transmise de façon stable au cours des générations cellulaires pour constituer des organes et tissus fonctionnels. Ce phénomène est représenté par l'épigénétique.

L'épigénome est une interface entre la génétique et l'environnement, quand la plasticité de l'épigénétique code un gène qui est supposé être stable pour exprimer un phénotype $[8,9]$. L'épigénétique est ce matériel sensé moduler des anomalies génétiques adaptant la lecture du code génétique et corriger des expressions pathogènes, mais l'épigénétique altérée peut aboutir à l'expression de certaines pathologies en détournant une séquence non altérée $[10,11]$. L'épigénétique est influencée contrairement à la génétique par l'environnement comme la diététique, l'inflammation, le stress oxydatif, les modifications métaboliques et les toxines $[12,13]$. L'atteinte épigénétique peut être exprimée physiopathologiquement par la méthylation de l'ADN, les modifications des histones et l'interférence ARN/ADN.

La formation des protéines intègre trois étapes; la transcription de l'ADN en ARN inverse puis en ARN messager pour former la chaines d'acides aminés. Un gène peut coder en réalité plusieurs séquences d'acides aminés qui dépend des zone promotrices de l'emplacement des exons le fragment exprimé alors que les introns est un fragment non codé. Ce polymorphisme est décrit au cours de l'insertion délétion au sein de l'intron 16 du gène codant pour l'enzyme de conversion décrite dans la progression de l'insuffisance rénale chronique chez des enfants japonais [14,15]. L'embryologiste écossais Conrad Waddington, dans les années 1940, désigne en effet l'épigénétique comme le lien entre le génotype (l'ensemble des gènes) et le phénotype (l'ensemble des caractères exprimés).

Aujourd'hui, on sait que non seulement nos caractères dépendent de ces gènes hérités, mais aussi des marques épigénétiques portées par la chromatine ; elle est héritable au cours des divisions cellulaires ou de la méiose. En effet, l'environnement, normal ou pathologique, en fournissant des signaux, est à même de modifier le modèle d'expression d'une cellule, puis peut être éventuellement transmis aux descendants.

En Suède, les généticiens se sont intéressés à un village bien particulier où la qualité des récoltes a été consignée avec soin, de la fin du XVIIIe et au début du XIXe siècle. L'alimentation des grands-pères qui ont connu de très bonnes récoltes (surnutrition) ou de très maigres récoltes (sous-nutrition) semble ainsi avoir des répercussions sur la santé de leurs petitsenfants. D'un côté, la malnutrition du grand-père est associée à un risque quatre fois plus grand de décéder d'un diabète que dans la population générale. D'un autre côté, la surnutrition des grands-pères entraîne sur deux générations une espérance de vie diminuée, de six ans en moyenne, par rapport aux enfants et petits-enfants de grands-parents qui ont enduré de mauvaises récoltes. Toutes ces modifications épigénétiques sont déclenchées par des schémas préprogrammés, enfouis dans les gènes, résume ainsi Saadi Khochbin.

$\mathrm{Au}$ sein de l'ADN humain seul $4 \%$ du code génétique est décrypté et produit les protéines. Les changements d'un seul nucléotide sont fréquents et sont connus sous le nom de polymorphismes nucléotidiques simple «SNPs» qui en général se situent dans les régions non codantes et n'impactent pas le morphotype, dans le cas contraire si ces modifications ont lieu au sein des séquences codantes, elles affectent la constitution biologique des protéines et se traduit par la manifestation de pathologies.

Depuis le séquençage du génome humain en 2004, la diversité du message génétique a révélé l'existence de variantes c'est-à-dire d'un changement du message génétique correspondant le plus souvent à la substitution d'une base par une autre à une localisation définie (1). Ces variantes dénommées SNP (Single Nucleotide Polymorphisms) sont bialléliques c'est-à-dire que le changement du message est présent à l'état homozygote ou hétérozygote. Les fréquences des génotypes, par exemple pour un SNP donné : CC, ou CT ou TT, ou bien des allèles (ici $\mathrm{C}$ ou T) est comparée ; et si un génotype ou un allèle est plus fréquemment observé chez les malades que chez les témoins, le SNP est considéré comme associé avec la maladie. Il existe plus de 14 millions de SNP dans le génome humain, dont environ 3 millions sont différents lorsque l'on compare la séquence génomique de deux individus non apparentés pris au hasard. Parmi ces SNP, environ 7 millions représentent un degré de polymorphisme assez fréquent puisque l'allèle mineur est présent chez plus de $5 \%$ des individus.

À partir de ces progrès, les scientifiques ont pu réaliser des études d'association sur l'ensemble de ces SNP, c'est-à-dire comparer la fréquence génotypique de chaque variant entre une population témoin et une population atteinte d'une pathologie. En 2005 fut ainsi publiée la première étude d'association utilisant un grand nombre de variantes génétiques et dénommée ainsi GWAS pour Genome-wide Association Study [16]. À la fin de l'année 2011, le catalogue international des études de GWAS répertoriait environ 5000 SNP associés à des pathologies diverses et issues d'environ un millier de publications.

En effet, la plupart des maladies multifactorielles comme le diabète, l'hypertension artérielle, la maladie d'Alzheimer ou les maladies inflammatoires chroniques de l'intestin ont une composante héréditaire. Cette hérédité est polygénique en ce sens qu'elle résulte de la combinaison de l'effet de nombreux 
variants avec d'autres facteurs liés à l'effet de l'environnement. Dans la plupart des maladies fréquentes étudiées à l'heure actuelle par les GWAS, de nombreux SNP ont été identifiés expliquant au total entre 10 et $30 \%$ de l'héritabilité de chacune de ces maladies. Dans la majorité des cas, les SNP identifiés représentent un effet faible dans le calcul du risque (Odds Ratio entre 1,10 et 1,30). Ce résultat est lié à l'analyse des tag-SNP qui pour la plupart ont une fréquence allélique supérieure à $5 \%$. Les puces actuelles et les données obtenues par l'étude de SNP rares (fréquence < $5 \%$ ), vont permettre de poursuivre l'identification de nouveaux gènes contribuant au risque des maladies multifactorielles.

\section{LA METHYLATION DE L'ADN ET MALADIES CARDIO- VASCULAIRES}

Les premiers travaux sur la relation des modifications épigénétiques et les anomalies morphologiques ont été conduits dans le domaine de la cardiologie [17], pour établir la relation entre les phénomènes épigénétique dans l'apparition des maladies cardiovasculaires et leurs évolution, passant par la genèse d'une maladie comme l'hypertension artérielle ou les atteintes coronariennes, pour définir les micro-ARN circulants comme biomarqueurs.

La méthylation des brins d'ADN est une modification épigénétique assez stable qui est définie par insertion du méthyl au sein du 5 ème carbone du groupe cytosine du dinucléotide " $C G$ » chez les mammifères. L'intérêt de la méthylation est de moduler l'expression de l'information de l'ADN à tout le processus de transcription avec un rôle assez crucial dans l'inactivation du chromosome X, éviter la répétition afin d'éviter les évènements pathogènes. Les modifications de l'ADN ainsi que les anomalies de méthylation ont été décrites dans la carcinogénèse et l'athérogènese. Citons comme exemple la relation étroite entre le taux d'homocystéine sérique de maladies cardiovasculaires et la méthylation du complexe « $C G$ » du brin d'ADN [18].

Récemment, Baccarelli a pu mettre en évidence une relation étroite entre la méthylation du brin d'ADN et les évènements cardiovasculaires comprenant les évènements ischémiques [19], le rôle protecteur de l'ostéogène contre l'athérogènese en se liant aux récepteurs ESR1 et ESR2. Un défaut d'expression de ces récepteurs est lié à la méthylation des gènes promoteurs sur le brin d'ADN de ces mêmes récepteurs [20]. L'expression de l'hypertension artérielle n'a pas échappé en partie aux modifications épigénétiques ; ainsi le gène codant la 11 beta-hydroxysteroide dehydrogénase 2 (HSD11B2) enzyme qui convertit la cortisol en cortisone; induit par sa méthylation une baisse du fonctionnement de l'enzyme liée à une augmentation du rapport cortisol/cortisone; rapport lié significativement à l'apparition d'une hypertension artérielle [21]. D'autres études se sont intéressées au promoteurs du gènes qui produisent les co-transporteurs $\mathrm{Na}, \mathrm{K}, \mathrm{Cl}$ au niveau rénal; ainsi Lee et collaborateurs ont pu détecter le gène qui régule l'expression de ces pompes alors qu'une hypométhylation influencée principalement par l'environnement et l'alimentation contribuerait au développement de l'hypertension par réabsorption excessive de sodium [22].

\section{LA GÉNÉTIQUE AU COURS DES MALADIES RÉNALES}

Les travaux sur la génétique au cours des néphropathies monogéniques ou maladies dues à des mutation d'un seul gène, ce raisonnement est valable pour les néphropathies polykystiques autosomiques dominantes (ADPKD) ou récessives (ARPKD), le syndrome d'Alport secondaire à une mutation dominante ou récessive sur le gène $X$, ou des pathologies multisystémiques dont les manifestation souvent sévère comme le syndrome de Bardet-Biedl [23]; ces maladies sont référencées dans les associations européennes rénales de hildebrandt, ou association de dialyse et transplantation (ERA-EDTA) [24,25].

Les pathologies dites polygéniques multifactorielles, quelque soit leur expression et leur physiopathologie, dépendent de l'interaction polygénique influencée probablement par des facteurs environnementaux. Ce lien entre complexe phénotypique et codage génétique est mis en évidence à l'aide de deux procédés soit via les études des associations de gènes candidats «GWAS » en comparant des sujets sains et sujets atteints ou études cas par cas à la recherche de différences entre groupes de patients. Les études d'association de gènes prennent naissance sur une base d'hypothèse de relation nécessitant un nombre d'individus souvent faible avec un nombre de gênes étudiés réduit dont le rôle phénotypique déjà suggéré et les effets biologiques sont établis sur des bases théoriques solides. L'exemple d'étude du gène candidat serait l'étude d'interaction du gène codant pour le chemokine receptor 5 (CCR5) et un taux de CRP élevé chez 603 sujets dialysés pour conclure que le CCR5x32 module l'état inflammatoire et a un impact sur la mortalité chez les insuffisants rénaux terminaux [26], hypothèse revue au cours d'une cohorte suédoise qui affirme qu'une délétion du gène protège et atténue les effets inflammatoires sur la mortalité quelque soit la cause [27].

Au cours des GWAS le génome est étudié de manière plus large, sans hypothèse préalable, dépendant de l'étude d'une population très importante souvent en méta-analyse; ainsi au cours d'une étude de consortium sur association du génomes à la pression artérielle chez 200.000 individus européens ont révélé 16 nouveaux locus associés à l'hypertension artérielle [28].

3000 papiers scientifiques ont été publiés sur la génétique au cours des pathologies rénales depuis les années 1980, cependant il y a peu de cohortes et les résultats sont assez hétérogènes. Même si des gènes sont plus étudiés que d'autres comme les travaux sur «UMOD » sont plus solides, il $\mathrm{y}$ a de nombreux travaux qui méritent une certaine attention selon le type d'atteinte rénale.

\section{Maladies rénales chroniques}

Köttgen et al. [29] ont pu mettre en évidence au cours d'une étude comprenant 19877 individus des SNPs dans le génome, le plus connu serait le gène UMOD qui code l'uromoduline exclusivement exprimée au niveau rénal. La concentration de cette uromoduline urinaire dépend de l'expression du gène ; elle est liée significativement au risque de développer une maladie rénale dans les 10 années. Il a donc été suggéré que rs429393 codant pour une uromoduline altérée représenterait un marqueur diagnostique [30].

En plus du gène UMOD, d'autres découvertes du consortium de maladies rénales chronique génétiques ont pu identifier PRKAG2, locus étudié chez une population de plus de 5000 sujets insuffisants rénaux, [31] qui code la production de l'adénosine monophosphate, protéine kinase : même si le rôle est moins clair au cours des néphropathies chroniques que pour le gène UMOD.

$\mathrm{Au}$ cours des maladies lithiasiques, thorleifsson et associés [32] ont identifié CLDN14, Claudin 14 exprimée dans le rein 
qui régule la perméabilité paracellulaire au niveau des tight junctions. Un défaut d'expression du gène est lié non seulement à une fréquence importante des lithiases mais aussi à la baisse de la densité osseuse et la dérégulation de l'homéostasie du calcium [33],

$\mathrm{Au}$ cours des hyalinose segmentaires et focales le rôle de plusieurs gènes a été identifié, chez plusieurs individus de générations différentes [32,34]. Chez une population d'afroaméricains, a été établi que cette mutation autosomique dominante s'exprime au sein des podocytes [35].

\section{Evaluation de la fonction rénale}

Plusieurs locus SHROOM3, GATM-SPATA5L1, STC1 analysés ont un rôle dans la morphogénèse épithéliale au cours du développement rénal [36]. STC1 code pour stanniocalcin 1, exprimée largement au sein du néphron [37], régule les concentrations de calcium intracellulaire qui a un rôle de cytotprotection et un rôle anti-inflammatoire. L'anomalie d'expression du gène est décrite au cours des glomérulonéphrites [38].

La néphrogénèse dépend aussi des locus ALMS1, VEGFA and DACH1. Plus précisément on peut relier la fonction podcytaire aux gènes DAB2 et VEGFA, l'angiogenèse au VEGFA, au transport des solutés liés aux gènes SLC7A9 et SLC34A1. Ces mêmes gènes sont incriminés par défaut de leur expression à plusieurs néphropathies [39].

Le débit de filtration glomérulaire évalué par la créatinine ou la cystatine $C$ dépend étroitement de l'expression des locus cystatine C (CST3) et cystatine-9 (CST9) [39-41].

A ce jour, 20 régions génomiques ont été découvertes au cours de travaux incluant plus 65000 individus. Le locus p43.13 est lié à la fonction rénale (LASS2, GCKR, NAT8/ALMS1, TFDP2, DAB2, SLC34A1, VEGFA, PRKAG2, FAM122A/PIP5K1B, ATXN2, DACH1, UBE2Q2/FBX022, et SLC7A9). 7 locus sont responsables de la production de la créatinine (CPS1, SLC22A2, TMEM60, WDR37, SLC6A13, WDR72, et TBX2/BCAS3). Ces données sont traitées des études qui sont toujours en cours pour identifier les facteurs de influençant l'évaluation de la fonction rénale $[40,41]$

\section{Insuffisance rénale aigue}

Le gène pax2 est crucial pour le développement des reins et le développement des voies urinaires et la voie de reproduction [42]. Un défaut d'expression du gène est lié chez la souris à une agénésie rénale et génitale [43]. Chez l'homme la mutation du gène se manifeste par le syndrome colobome comprenant une dysplasie voire aplasie de l'appareil urinaire avec reflux urétérovasical [44]. Un autre rôle suggéré dans la régénération des cellules épithéliales est qu'un défaut d'expression limiterait la récupération de la fonction rénale devant les agressions [45].

\section{Albuminurie}

La micro-albuminurie est un facteur de progression de la maladie rénale et un facteur de risque cardiovasculaire, [46], sa présence au niveau des urines a été rapportée dans plusieurs méta-analyses à l'expression du gène CUBN chez les Européens et Afro-Américains. Le gène CUBN est associé au risque progression de l'insuffisance rénale terminale [47] ; la cubiline codée par ce gène est largement exprimée dans les tubules rénaux proximaux [47]. Son expression entrainerait une dysfonction d'endocytose et donc participe dans la pathogénèse de la néphropathie diabétique. [46]

\section{Néphropathie diabétique}

La réflexion sur l'association de la génétique et la néphropathie diabétiques, est née après publication de cas de diabétiques normotendus, non ou peu micro albuminuriques qui développent une insuffisance rénale.

L'étude des reins des diabétiques retrouve la présence non régulée de $\mathrm{NF} \mathrm{kb}$ avec défaut de transcription du gène correspondant [48]. Les travaux sur l'épigénétique décrivent les effets de l'hyperglycémie sur la structure des histones qui se traduit par une modification de l'expression du gène vasculaire [49); et sur la fonction des monocytes [50]. D'autres travaux ont pu corréler la modification de l'ARN messager et l'expression des cellules épithéliales, podocytaires et mésangiales [51]

Les premiers gènes identifiés chez les Japonais indiquent que ELMO1 (engulfment and cell motility 1) comme gène conférant probablement une susceptibilité à développer une néphropathie diabétique type2 [52]. D’autres gènes ont été associés comme étant potentiellement des facteurs de risque de développer une néphropathie diabétique FRMD3, CARS (cystéinyl-tRNA synthétase) et ACACB (acetyl coznzyme Acarboxylase) [53]

\section{Néphropathie à IgA}

La néphropathie à IgA bien répandue en Europe et en Asie, peut être liée à l'anomalie d'expression de plusieurs gènes essentiellement HORMAD2 et gène $\mathrm{CFH}$ significativement au cours de la maladie $[54,55]$

\section{Perspectives et limites de la génétique}

Les variations au sein du génome et de l'épigénome engendrant des pathologies; leur compréhension à intérêt diagnostique mais aussi thérapeutique. En effet, agir sur des facteurs comme: l'hygiène de vie, une supplémentation alimentaire peut avoir un impact thérapeutique [56,57] pour aboutir dans un futur à une médecine personnalisée.

A l'inverse, une étude publiée récemment qui avait pour objectif d'évaluer le risque de développer une insuffisance rénale chronique stade 3 au bout de 10 ans chez 2489 personnes d'origine européenne issus de la de la cohorte de framingham n'a pas permis de montrer l'impact de 16 SNP comme facteur de risque au dans un modèle multifactoriel comprenant l'âge, le sexe, la fonction rénale, l'hypertension, le diabète et la protéinurie. Il ne semble pas que les antécédents familiaux d'insuffisance rénale chronique ne soit un facteur de risque [58,59].

\section{CONCLUSION}

Il est donc actuellement difficile d'intégrer les données génétiques et épigénétique dans notre pratique clinique quotidienne pour la prise en charge des maladies multifactorielles. Il n'est pas encore possible de prédire l'apparition d'affections systémiques après lecture du génome. Il serait cependant intéressant de suivre l'évolution des travaux afin de peaufiner la lecture du génome qui permettrait le médecin d'agir plutôt dans l'évolution des pathologies et d'être plus efficace en prévention primaire.

Déclaration d'intérêts : l'auteur ne déclare aucun conflit d'intérêt en rapport avec cet article. 


\section{RÉFÉRENCES}

1. Sarnak MJ, Levey AS, Schoolwerth AC, et al. Kidney disease as a risk factor for development of cardiovascular disease: a statement from the American Heart Association councils on kidney in cardiovascular disease, high blood pressure research, clinical cardiology, and epidemiology and prevention. Circulation $108,2154-2169$ (2003).

2. Weiner DE1, Tighiouart $\mathrm{H}$, Amin MG, et al. Chronic kidney disease as a risk factor for cardiovascular disease and all-cause mortality: a pooled analysis of community-based tudies. J Am Soc Nephrol. 2004 May;15(5):1307-15.

3. Dennis C. Epigenetics and disease: altered states. J Am Soc Nephrol. 2003;14:2902-7

4. Baldwin DS. Chronic glomerulonephritis: nonimmunologic mechanisms of progressive glomerular damage. Kidney Int. 1982; 21:109-20.

5. Qin $W$, Chung $A C$, Huang $X R$, et al. TGF- $\beta / S m a d 3$ signaling promotes renal fibrosis by inhibiting miR-29. J Am Soc Nephrol 22: 1462-74.

6. Melamed ML, Bauer C, Hostetter TH. eGFR : is it ready for early identification of CKD? Clin J Am Soc Nephrol 2008;3:1569-1572.

7. Rouse RL, Zhang J, Stewart SR, et al. Comparative profile of commercially available urinary biomarkers in preclinical drug-induced kidney injury and recovery in rats. Kidney Int 2011; 79: 1186-1197.

8. Bonasio R, Tu S, Reinberg D. Molecular signals of epigenetic states. Science. 2010; 30:612-6.

9. Franks PW, Nettleton JA. Invited commentary: gene $x$ lifestyle interactions and complex diseasetraits-inferring cause and effect from observational data, sine qua non. Am J Epidemiol. 2010;172:992-7.

10. Lawson HA, Zelle KM, Fawcett GL, et al. Genetic, epigenetic, and gene-by-diet interaction effects underlie variation in serum lipids in a $L G / J x S M / J m u r i n e$ model. J Lipid Res. 2010; 51:2976-84.

11. Feinberg AP. Epigenetics at the epicenter of modern medicine. JAMA. 2008; 299:1345-50.

12. Luch A. Nature and nurture-lessons from chemical carcinogenesis. Nat Rev Cancer. 2005; 5:113

13. Robertson KD, Wolffe AP. DNA methylation in health and disease. Nat Rev Genet. 2000; 1:11-9.

14. Nakayama $Y$, Nonoguchi $H$, Kohda $Y$, et al. Different mechanisms for the progression of CKD with ACE gene polymorphisms.Nephron Clin Pract. 2009;111(4):c240-c246.

15. Elshamaa MF, Sabry SM, Bazaraa HM, et al. Genetic polymorphism of ACE and the angiotensin II type1 receptor genes in children with chronic kidney disease. J Inflamm (Lond). 2011;8(1):20.

16. Klein RJ, Zeiss $\mathrm{C}$, Chew $\mathrm{EY}$ et al. Complement factor $\mathrm{H}$ polymorphism in agerelated macular degeneration. Science, 2005, 308: 385-389.

17. Jones, P.A, Laird, P.W. Cancer epigenetics comes of age. Nat. Genet. 21 (2), 163-167.

18. Handy, D.E., Castro, R., Loscalzo, J. Epigenetic modifications: basic mechanisms and role in cardiovascular disease. Circulation 123 (19), 21452156.

19. Baccarelli, A, Wright, R, Bollati, V, et al. Ischemic heart disease and stroke in relation to blood DNA methylation. Epidemiology 21 (6), 819-828.

20. Zuo, X., Shen, L., Issa, et al. 15-Lipoxygenase-1 transcriptional silencing by DNA methyltransferase-1 independently of DNA methylation. FASEB J. 22 (6), 1981-1992.

21. Friso, S., Pizzolo, F., Choi, Set al. Epigenetic control of 11 betahydroxysteroid dehydrogenase 2 gene promoter is related to human hypertension. Atherosclerosis 199 (2), 323-327.

22. Lee, H.A, Baek, I, Seok, Y, et al. Promoter hypomethylation upregulates Na+$\mathrm{K}+-2 \mathrm{Cl}$ _ cotransporter 1 in spontaneously hypertensive rats. Biochem. Biophys. Res. Commun. 396 (2), 252-257.

23. Robert J. Klein. Complement Factor H Polymorphism in Age-Related macular degeneration. Science 2005;308:385-389.

24. Hildebrandt F. Genetic kidney diseases. Lancet. 2010;375 (9722):1287-1295

25. ERA-EDTA Working Group on Inherited Kidney Disorders. WGiKD. Available at: http://www.eradta.org/wgikd/ERAEDTA working_group_on_Inherited_kidne y_disorders.htm. Accessed Feb 22, 2012.
26. Muntinghe $\mathrm{FL}$, Verduijn $\mathrm{M}$, Zuurman $\mathrm{MW}$, et al. CCR5 deletion protects against inflammation-associated mortality in dialysis patients. J Am Soc Nephrol. 2009;20(7):1641-1649.

27. Muntinghe $\mathrm{FL}$, Vegter $\mathrm{S}$, Verduijn $\mathrm{M}$, et al. Using a genetic, observational study as a strategy to estimate the potential cost-effectiveness of pharmacological CCR5 blockade in dialysis patients. Pharmacogenet Genomics. 2011; 21(7) : 417-425.

28. Ehret GB, Munroe PB, Rice KM, et al. Genetic variants in novel pathways influence blood pressure and cardiovascular disease risk. Nature. 2011;478(7367):103-109

29. Kottgen A, Glazer NL, Dehghan A et al. Multiple loci associated with indices of renal function and chronic kidney disease. Nat Genet 2009; 41:712-717.

30. Kottgen A, Hwang SJ, Larson MG et al. Uromodulin levels associate with a common UMOD variant and risk for incident CKD. J Am Soc Nephrol 2010; 21: 337-344.

31. Kottgen A, Pattaro C, Boger CA, et al. New loci associated with kidney function and chronic kidney disease.Nat Genet. 2010;42(5):376-384.

32. Kopp JB, Smith MW, Nelson GW, et al. MYH9 is a major-effect risk gene for focal segmental glomerulosclerosis. Nat Genet. 2008;40(10):1175-1184.

33. Wilcox ER, Burton $\mathrm{Q} L, \mathrm{Naz} S$, et al. Mutations in the gene encoding tight junction claudin-14 cause autosomal recessive deafness DFNB29. Cell. 2001;104(1):165-172.

34. Kao WH, Klag MJ, Meoni LA, et al. MYH9 is associated with nondiabetic endstage renal disease in African Americans. Nat Genet. 2008;40(10):1185-1192.

35. Dong F, Li S, Pujol-Moix N, et al. Genotypephenotype correlation in MYH9related thrombocytopenia. Br J Haematol. 2005;130(4):620-627.

36. Lee C, Le MP, Wallingford JB. The shroom family proteins play broad roles in the morphogenesis of thickenedepithelial sheets. Dev Dyn. 2009;238(6):14801491.

37. Chang $A C$, Janosi J, Hulsbeek $M$, et al. A novel human CDNA highly homologous to the fish hormone stanniocalcin.Mol Cell Endocrinol. $1995 ; 112(2): 241-247$

38. Huang L, Garcia G, Lou Y, et al. Anti-inflammatory and renal protective actions of stanniocalcin-1 in a model of anti-glomerular basement membrane glomerulonephritis. Am J Pathol. 2009;174(4):1368-1378.

39. O'Seaghdha CM, Fox CS. Genome-wide association studies of chronic kidney disease: what have we learned? Nat Rev Nephrol. 2012; 8: 89-99.

40. Pattaro C, Kottgen A, Teumer A et al. Genome-wide association and functional follow-up reveals new loci for kidney function. PLoS Genet 2012; 8:e1002584.

41. Marian AJ, Belmont J. Strategic approaches to unraveling genetic causes of cardiovascular diseases. Circ Res 2011; 108: 1252-1269.

42. Robson E.J, He S.J, Eccles M.R. APANorama of PAX genesin cancer and development. Nat Rev Cancer6,52-62.

43. Soofi, A.,Levitan,I.,Dressler, et al. Two novel EGFP insertionalle lesreveal unique aspects of Pax2 function in embryonic and adult. kidneys Dev Biol 365, 241-250.

44. Bower,M.,Salomon,R.,Allanson,et al. Update of PAX2 mutations in renal coloboma syndrome and establishment of a locus-specific database. Hum Mutat 33,457-466.

45. Kusaba,T.,Lalli,M.,Kramann,R.,Kobayashi,A.,Humphreys,B.D.,2014.Differentiated kidneye pithelial cells repair injured proximal tubule. Proc Natl Acad. Sci. U.S.A.111,1527-1532.

46. Boger CA, Chen MH, Tin A et al. CUBN is a gene locus for albuminuria. JAm Soc Nephrol 2011; 22: 555-570.

47. Reznichenko A, Snieder $\mathrm{H}$, van den Born J et al. CUBN as a novel locus for endstage renal disease: insights from renal transplantation. PLoS One 2012; 7: e36512.

48. Woroniecka, K.I Park, Mohtat. Transcriptome analysis of human diabetic kidney disease. Diabetes 2011, 60, 2354-2369.

49. Tonna, S ,El-Osta, A, Cooper M.E, et al. Metabolic memory and diabetic nephropathy: Potential role for epigenetic mechanisms. Nat. Rev. Nephrol. 2010, 6, 332-341.

50. El-Osta A, Brasacchio D, Yao D, et al. Transient high glucose causes persistent epigenetic changes and altered gene expression during subsequent normoglycemia. J. Exp. Med. 2008, 205, 2409-2417. 


\section{DOSSIER THÉMATIQUE}

51. Wang $B$, Herman-Edelstein, $M, K o h P$, et al. E-cadherin expression is regulated by miR-192/215 by a mechanism that is independent of the profibrotic effects of transforming growth factor-beta. Diabetes. 2010 Jul; 59(7): 1794-1802

52. Shimazaki A, Kawamura $Y$, Kanazawa $A$ et al. Genetic variations in the gene encoding ELMO1 are associated with susceptibility to diabetic nephropathy.Diabetes 2005; 54: 1171-1178.

53. Sandholm N, Salem RM, McKnight AJ et al. New susceptibility loci associated with kidney disease in type 1 diabetes. PLoS Genet 2012; 8:e1002921.

54. Palmer ND, Freedman BI. Insights into the genetic architecture of diabetic nephropathy. Curr Diab Rep 2012; 12: 423-431.

55. Feehally J, Farrall $M$, Boland $A$ et al. HLA has strongest association with genome-wide search for linkage of estimated glomerular filtration rate (eGFR) in the Family Investigation of Nephropathy and Diabetes (FIND). PLOS ONE 8: e81888, 2013.
56. Ingrosso D, Cimmino A, Perna AF, Masella L, De Santo NG, De Bonis ML, et al. Folate treatment and unbalanced methylation and changes of allelic expression induced by hyperhomocysteinaemia in patients. The lancet. Volume 361; Number 9370 ; p1665-1754.

57. Oommen AM, Griffin JB, Sarath G, Zempleni J. Roles for nutrients in epigenetic events. J NutrBiochem. 2005; 16:74-7.

58. Conall M. Performance of a Genetic Risk Score for CKD Stage 3 in the General Population ». American Journal of Kidney Diseases 59, no. 1 (janvier 2012): $19-$ 24.

59. William M, et al.Association of Family History of ESRD, Prevalent Albuminuria, and Reduced GFR With Incident ESRD. American Journal of Kidney Diseases 59, no. 1janvier 2012: 25-31

Cet article a été publié dans le « Batna Journal of Medical Sciences » BJMS, l'organe officiel de "I'association de la Recherche Pharmaceutique - Batna»

Le contenu de la Revue est ouvert "Open Access » et permet au lecteur de télécharger, d'utiliser le contenu dans un but personnel ou d'enseignement, sans demander l'autorisation de l'éditeur/auteur.

Avantages à publier dans BJMS :

- Open access : une fois publié, votre article est disponible gratuitement au téléchargement

- Soumission gratuite : pas de frais de soumission, contrairement à la plupart des revues « Open Access "

- Possibilité de publier dans 3 langues : français, anglais, arabe

- Qualité de la relecture : des relecteurs/reviewers indépendants géographiquement, respectant l'anonymat, pour garantir la neutralité et la qualité des manuscrits.

Pour plus d'informations, contacter BatnaJMS@gmail.com

ou connectez-vous sur le site de la revue : www.batnajms.com 


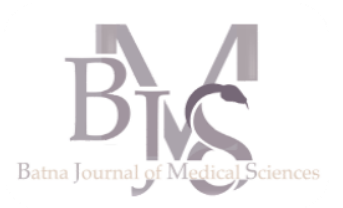

\title{
Épigénétique et pathogénèse du diabète type 2
}

\author{
Epigenetics and pathogenesis of type 2 diabetes
}

\author{
Adel Gouri ${ }^{1}$, Mohamed Bouchareb ${ }^{2}$, Aoulia Dekaken ${ }^{3}$, Ahmed Aimen Bentorki ${ }^{4}$, \\ Mohamed Chefrour ${ }^{5}$, Régis Guieu ${ }^{5}$, Sadek Benharkat 1
}

1. Laboratoire de Biochimie Médicale, CHU IBN ROCHD, Annaba, Algérie.

2. Laboratoire de Microbiologie, EPH IBN ZOHR, Guelma, Algérie.

3. Service de Médecine interne, EPH Prof EL OKBI, Guelma, Algérie.

4. Laboratoire de Microbiologie, CHU DORBAN, Annaba, Algérie

5. Laboratoire de Biochimie, Hôpital Universitaire de La TIMONE, AP-HM, Marseille France.

\section{Correspondance à :}

Adel GOURI

pharmagor@gmail.com

DOI :https://doi.org/10.48087/ BJMStf.2015.2113

Il s'agit d'un article en libre accès distribué selon les termes de la licence Creative Commons Attribution International License (CC BY 4.0), qui autorise une utilisation, une distribution et une reproduction sans restriction sur tout support ou format, à condition que l'auteur original et la revue soient dûment crédités.

\section{RÉSUMÉ}

Le diabète a atteint des proportions épidémiques dans le monde entier. Des éléments de preuves suggèrent qu'il y a une interaction complexe entre les gènes et l'environnement pouvant ainsi jouer un rôle majeur dans la pathogénie multifactorielle de cette maladie et ses complications évoquant ainsi, un résultat de l'implication des facteurs épigénétiques. De récentes études montrent que les facteurs épigénétiques, y compris la méthylation de l'ADN et les modifications de l'histone, peuvent affecter la sensibilité ainsi que l'évolution des complications du diabète de type 2. Toutefois, nos connaissances sur les mécanismes moléculaires reliés aux facteurs environnementaux et le diabète de type 2 restent limitées.

Mots-clés : Epigénétique, Diabète de Type 2, Méthylation de l'ADN, Histones

\begin{abstract}
Diabetes has reached epidemic proportions throughout the world. Increasing evidence suggests that complex interactions between genes and the environment might play a major role in the pathogenesis of this multifactorial disease and its complications, and this might be a result of the involvement of epigenetic factors. Recent studies show that epigenetic factors, including DNA methylation and histone modification may affect the susceptibility for type 2 diabetes and the progression of their complications. However, molecular mechanisms linking environmental factors and type 2 diabetes still remain limited.
\end{abstract}

Keywords: Epigenetics, Type 2 diabetes, DNA methylation, Histones

$$
\begin{aligned}
& \text { الوراثة اللاجينية والآلية المرضية في مرض السكري من النوع } 2 \\
& \text { الملخص: } \\
& \text { إن مرض السكري قد بلغ مستويات وبائية مرتفعة في جميع أنحاء العالم، وتثير أدلة متز ايدة على أن التفاعلات المعقدة بين الجينات والبيئة قد }
\end{aligned}
$$

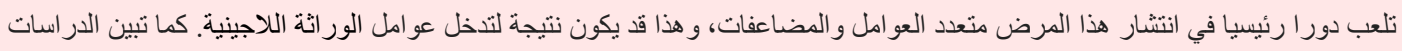

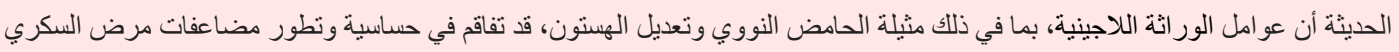

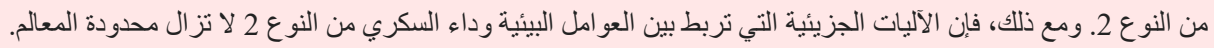

$$
\begin{aligned}
& \text { الكلمات المفتاحية: الور اثة اللاجينية، مرض السكري من النوع 2، مثيلة الحامض النووي، الهستون. }
\end{aligned}
$$

\section{INTRODUCTION}

À l'orée du 21e siècle, le diabète s'impose comme l'un des problèmes majeurs de santé publique au niveau mondial, la prévalence du diabète chez les adultes était estimée à 366 millions en 2011; en 2030 elle avoisinera les 552 millions. Le diabète de type 2 est la forme prédominante et représente au moins $90 \%$ des cas. Dans les pays à faible et moyen revenu, vivent $80 \%$ des personnes atteintes de diabète dont la classe d'âge la plus touchée est comprise entre 40 et 59 ans. [1, 2]

Le diabète de type 2 , connu par sa coexistence polygénique et multifactorielle est une maladie caractérisée par une hyperglycémie et une modification du métabolisme lipidique, due à l'altération de la sécrétion d'insuline par les cellules- $\beta$ du pancréas. Aujourd'hui, il est bien établi que des combinaisons de risques entre les facteurs non génétiques (tels que : l'obésité, l'inactivité physique, et le vieillissement) et les facteurs génétiques influencent la sensibilité au diabète de type 2. Des études de "GenomeWide-Association" ont identifié plus de 40 polymorphismes associés à un risque accru de la maladie [3-7].

D'autre études récentes ont montré que les facteurs épigénétiques, y compris la méthylation de l'ADN et les modifications de 
l'histone, peuvent affecter également la sensibilité ainsi que l'évolution des complications du diabète de type 2 [8].

Toutefois, les mécanismes moléculaires reliés aux facteurs environnementaux et le diabète de type 2 restent limités. Cette mis au point permettra d'établir un aperçu sur les mécanismes épigénétiques impliqués dans le développement du diabète de type 2 .

\section{APERÇU SUR LES MÉCANISMES ÉPIGÉNÉTIQUES}

L'épigénétique est actuellement décrite comme l'étude des modifications de l'expression génétique qui se distingue non pas par la modification de la séquence d'ADN, mais en modifiant la méthylation de l'ADN et la rénovation de la chromatine. Au cours des dernières années, des progrès majeurs dans la compréhension des mécanismes épigénétiques ont démontré que ces derniers sont les acteurs clés dans plusieurs processus cellulaires, y compris la différenciation cellulaire, le vieillissement, la réplication et la réparation de l'ADN [9-12].

Les principaux mécanismes épigénétiques sont la méthylation de l'ADN, les modifications de l'histone et la modulation de la transcription et de la traduction des gènes par les Non-Coding RNAs, y compris les Micro RNAs (MiRNAs).

La méthylation de l'ADN est une modification génomique qui peut influencer l'activité génétique. Elle survient presque exclusivement sur les résidus de la cytosine au niveau des dinucléotides de types $C P G$, qui tendent à se regrouper dans des régions appelées "CpG îlot". La fonction principale de la méthylation de l'ADN est souvent utilisée pour réduire au silence ou réguler dans les régions de l'ADN où la transcription n'est pas souhaitée [13].

Les modifications des histones sont le résultat conformationnel de régulation transcriptionnelle de la chromatine qui modifie l'accès des promoteurs de facteurs de transcription. Ces modifications, intervenant par quatre types majeurs à savoir: l'acétylation, la méthylation, la phosphorylation, et l'ubiquitination; agissant ainsi sur l'interaction entre les histones, l'ADN et les protéines nucléaires : donc touchant le gène de transcription et réguler celui de la neutralisation ou d'expression [13]

Un troisième mécanisme implique l'expression de Non-coding RNAs court, cette expression peut conduire à la neutralisation en translation par la liaison spécifique et à une éventuelle dégradation de l'ARN transcrit. MicroRNAs (miRNAs) peut également réguler la méthylation de l'ADN et les modifications de l'histone [14]

\section{EPIGÉNÉTIQUE ET PATHOGÉNÈSE DU DIABÈTE DE TYPE 2}

Le terrain de recherche Epigénétique sur le diabète du type 2 (DT2) est à ces débuts.

Les rôles des mécanismes épigénétiques dans l'étiologie du diabète et de ses anomalies métaboliques tels que l'obésité, la dyslipidémie, l'hypertension, l'hyperglycémie sont mal défini. Les effets épigénétiques peuvent également être touchés par l'environnement, rendant ainsi ces mécanismes potentiellement pathogènes au cours des maladies multifactorielles entre autre le diabète de type 2 (Figure 1).
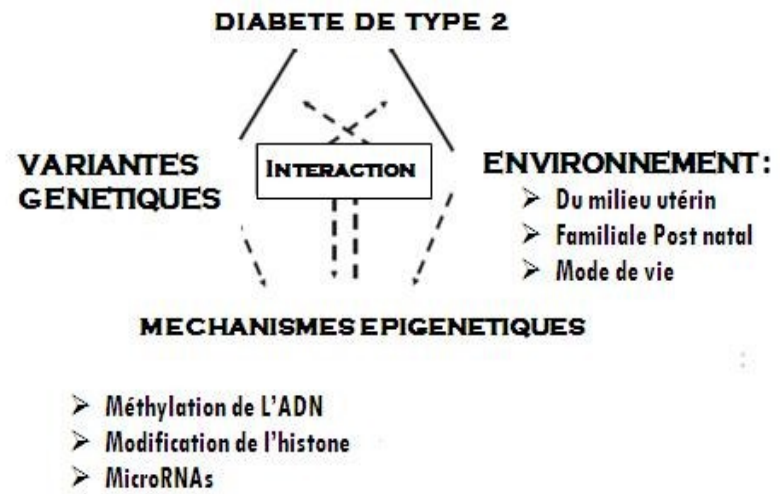

Figure 1: interactions entre facteurs génétiques et environnementaux dans la genèse du diabète.

Des évidences importantes impliquant le rôle des facteurs épigénétiques dans la pathogenèse du DT2 on été relevées à partir de l'analyse des données de plus de 12 million d'enregistrements de la base Medline [15]. Ces études ont prouvé que la méthylation de l'ADN et la chromatine sont les points clés impliqués dans le développement du DT2.

L'épigénétique du DT2 est le résultat d'interaction entre l'activation génique et l'épidémiologie, où l'activation du gène peut se faire soit par : la méthylation de l'ADN, la modification d'histone ou l'activation d'ARN ; cette activation pourrait être affectée par différents facteurs épidémiologiques à savoir : l'âge, l'obésité, la nutrition, l'activité physique, en outre il a été suggéré l'existence de liens entre les diverses expositions au cours de la vie intra-utérine (voire dès la fécondation) et le risque du diabète [16-18].

Les Mécanismes épigénétiques tels que la méthylation de l'ADN et la modification d'histone sont de plus en plus impliqués dans la transmission phénotypique et le développement de DT2. Dans les cellules de mammifères différenciées, l'ajout de groupements de méthyle de l'ADN se produit sur les résidus cytosines et ces modifications sont établies pour la plupart dans le contexte de cytosine guanine dinucléotides (CpGs), cette réaction est catalysée par différents membres d'une seule famille d'enzymes. La méthylation de l'ADN est souvent associée à la mise en silence du gène et contribue à l'inactivation du chromosome $\mathrm{X}$, ainsi que l'imprégnation génomique et la régulation transcriptionnelle des gènes spécifiques de tissu durant la différenciation cellulaire [16].

Bien que l'analyse de données ait suggéré un rôle des facteurs épigénétiques dans la pathogenèse du diabète de type 2 [19], il y a seulement un nombre limité d'études qui ont exploré les modifications épigénétiques dans les tissus cibles des patients atteints de diabète de type 2 .

Récemment, des chercheurs ont révèlé que l'épigénétique du DT2 humain implique le Peroxysome Proliférateur Active Récepteur Gamma Co-activateur 1 Alpha (communément appelé : PGC-1 $\alpha$, et codé par le PPARGC1A) et l'adaptation génique du métabolisme mitochondrial. De cette implication résulterait un déficit dans la sensibilité aux taux de glucose sanguins, suite à des dysfonctionnements mitochondriaux, qui se traduit par des fluctuations dans la production d'ATP et le stimulus nécessaire à la sécrétion d'insuline par la cellule- $\beta$ du pancréas. En plus, le niveau de méthylation d'ADN augmente le taux du Co-activateur transcriptionnel PGC1 $\alpha$ dans les îlots pancréatiques de malades atteints de DT2 comparativement à un groupe témoin apparenté de même insulino-résistance [20]. 
Toutefois, l'association entre le gène codé par le PPARGC1A et la sensibilité à l'insuline témoigne d'une expression génique dans les muscles squelettiques constatée lors des précédentes études dont le résultat est contradictoire. Une étude récente a établi une relation paradoxale entre la méthylation de l'ADN PPARGC1A et l'action d'insuline dans le muscle squelettique [21].

En plus, une analyse globale de la méthylation de l'ADN dans les muscles squelettiques, à révélé chez des personnes ayant des antécédents familiaux de DT2 une différence de méthylation de l'ADN des gènes impliqués dans la fonction musculaire ainsi que dans la voie de signalisation de l'insuline et du calcium [22].

En outre, un lien évident entre la modification d'histone et le métabolisme a été confirmé par le fait qu'une diminution de la fonction de déméthylase d'histone (JHDM2A) est génératrice de l'obésité et diminue l'expression des gènes métaboliques en cause, y compris le peroxisome proliferatoractivated receptor alpha (PPARA) et uncoupling protein 1 (UCP1). Idem à la méthylation de l'ADN, les modifications d'histone fournissent également un lien moléculaire entre un mode de vie sédentaire et le développement de T2D [19].

Récemment, Jufvas A et al. ont révélé une grande différence dans le niveau spécifique de méthylation de l'histone $\mathrm{H} 3$ des adipocytes chez les sujets diabétiques ou en surpoids par rapport aux sujets de poids normale et non diabétiques [23]. Il est maintenant évident que la contribution de la régulation épigénétique dans la manifestation des maladies métaboliques reste à élucider.

\section{EPIGÉNÉTIQUE ET COMPLICATIONS DIABÉTIQUES}

Le diabète et les troubles du métabolisme sont les principales causes de micro et macro complications vasculaires telles que l'athérosclérose, l'hypertension, la néphropathie, la rétinopathie et la neuropathie. Un événement majeur dans la progression des complications diabétiques est l'inflammation vasculaire engendrée par une expression accrue de gènes inflammatoires. Le stress oxydatif, la dyslipidémie et l'hyperglycémie ont été impliqués dans le développement des complications diabétiques [24].

\section{Complications cardiovasculaires}

Elles restent la principale cause de morbi-mortalité dans la population diabétique. Il est de plus en plus évident que l'hyperglycémie est le facteur majeur conduisant à ces complications. De récentes études ont proposé que l'hyperglycémie puisse induire des modifications épigénétiques de gènes impliqués dans l'inflammation vasculaire. Ces études ont révélé que le déterminant transcriptionnel; le facteur nucléaire (NF)- $\kappa B$, qui est facilement activées par les phases d'hyperglycémie, joue un rôle essentiel dans les complications vasculaires chez les diabétiques [24].

En outre, l'activation du NF-кB entraîne l'hyperstimulation de plusieurs molécules telle que la cytokine (MCP)-1 et les molécules d'adhésion aux cellules vasculaires (VCAM)-1, qui ont été largement étudiés dans l'athérosclérose [25].

Les mécanismes épigénétiques tels que la modification post transcriptionnelle des histones et la méthylation de l'ADN, jouent un rôle central de la régulation des gènes en affectant la structure et la fonction de la chromatine. De récentes études ont suggéré que l'hyperglycémie induit un changement dans la méthylation de l'ADN qui persiste dans l'état de la mémoire métabolique.

Le rôle de la méthylation de l'ADN dans la pathogenèse des maladies cardio-vasculaires n'est pas complètement élucidé. L'athérosclérose était associée à une hypo-méthylation globale des cellules vasculaires des muscles lisses (VSMCs) [24].

En plus, plusieurs études ont impliqué les miRNAs dans la pathogenèse du diabète. Toutefois, le rôle des miRNAs dans les complications vasculaires du diabète est moins étudié. Des preuves ont montré que les miRNAs peuvent affecter à la fois la fonction des cellules endothéliales (ECS) et des cellules vasculaires des muscles lisses (VSMCs) engendrant ainsi des lésions vasculaires [27-29].

\section{La néphropathie diabétique}

Dans la néphropathie diabétique, une fibrose tubulointerstitielle, due à une surexpression des protéines de la matrice extracellulaire telles que le collagène et les fibronectines, est lancée et soutenue par un certains nombres de facteurs, y compris la famille de TGF $\beta$. Cette famille de médiateurs inflammatoires est connue par une expression aberrante en mémoire métabolique, impliquant le TGF- $\beta$ comme un grand médiateur des événements épigénétiques dans la néphropathie diabétique [30,31].

\section{La rétinopathie diabétique}

Le rôle des mécanismes épigénétiques dans la pathogenèse de la rétinopathie diabétique a été récemment décrit. La première étude a examiné les moyens de contrôle du VEGF (significatif à la fois dans les stades précoces et tardifs de rétinopathie diabétique) par les micros ARN (miR-200b). La deuxième a révélé que l'activité des métalloprotéinases matricielles MMP2 et MMP9 provoque des dommages de l'ADN mitochondrial et une dégradation des membranes mitochondriales dans les cellules capillaires de la rétine qui à son tour induit son apoptose [31].

\section{CONCLUSION}

Le diabète est une maladie multifactorielle impliquant des interactions entre des facteurs génétiques et environnementaux. Des estimations alarmantes indiquent que la fréquence du diabète et les complications associées augmentent rapidement, d'où la nécessité de nouvelles stratégies pour limiter ces tendances. L'Epigénétique fournit un mécanisme qui peut expliquer l'étiologie du diabète et de la diversité des phénotypes dans la population générale. Bien qu'il y ait un appui pour le rôle de l'épigénétique dans la pathogenèse du diabète et de ses complications, les études sur les tissus humains auprès de diabétique restent limitées.

Le nombre de cibles thérapeutiques potentielles augmente à mesure que progressent les connaissances des mécanismes épigénétiques du développement du diabète. Cependant, le diabète étant un syndrome complexe et multifactoriel, son traitement doit prendre en compte non seulement les réalités physiologiques liées au terrain génétique, mais également les facteurs épigénétiques, nutritionnels et environnementaux.

Déclaration d'intérêts : l'auteur ne déclare aucun conflit d'intérêt en rapport avec cet article. 


\section{RÉFÉRENCES}

1. Waki H, Yamauchi T, Kadowaki T. The epigenome and its role in diabetes. Curr Diab Rep. 2012 Dec;12(6):673-85.

2. Magklara A, Lomvardas S. Epigenetics and Human Disease. N. Ahituv (ed.), Gene Regulatory Sequences and Human Disease, 2012, Springer Pages 253279.

3. Bluestone JA, Herold K, Eisenbarth G. Genetics, pathogenesis and clinical interventions in type 1 diabetes. Nature. 2010 Apr 29;464(7293):1293-300

4. Ziegler AG, Nepom GT. Prediction and pathogenesis in type 1 diabetes. Immunity (2010) 32:468-478.

5. MacFarlane AJ., Epigenetic Epidemiology of Type 1 Diabetes. K.B. Michels (ed.), Epigenetic Epidemiology, 2012 Springer (C).

6. Wren JD, Garner HR. Data-mining analysis suggests an epigenetic pathogenesis for type 2 diabetes. J Biomed Biotechnol; 2005(2):104-112.

7. Villeneuve LM, Reddy MA, Natarajan R.. Epigenetics: deciphering its role in diabetes and its chronic complications. Clin Exp Pharmacol Physiol. 2011 Jul;38(7):451-9.

8. Pinney SE, Simmons RA.. Epigenetic mechanisms in the development of type 2 diabetes. Trends Endocrinol Metab. 2010 Apr;21(4):223-9.

9. Ling C, Groop L. Epigenetics: A Molecular Link Between Environmental Factors and Type 2 Diabetes Diabetes. 2009 Dec;58(12):2718-25.

10. Kirchner $H$, Osler ME, Krook A, Zierath JR. Epigenetic flexibility in metabolic regulation: disease cause and prevention?. Trends Cell Biol. 2013 May;23(5):203-9

11. Lin J, Handschin C, Spiegelman BM. Metabolic control through the PGC-1 family of transcription coactivators. Cell Metab. 2005;1(6):361-370.

12. Nitert, M.D. et al. Impact of an exercise intervention on DNA methylation in skeletal muscle from first-degree relatives of patients with type 2 diabetes. (2012) Diabetes 61, 3322-3332.

13. Reddy MA, Natarajan R. Epigenetic mechanisms in diabetic vascular complications. Cardiovasc Res. 2011 Jun 1;90(3):421-9.

14. Brownlee M. . Biochemistry and molecular cell biology of diabetic complications. Nature. 2001;414:813-820.

15. Burke AP, Kolodgie FD, Zieske A, et al. Morphologic findings of coronary atherosclerotic plaques in diabetics: a postmortem study. Arterioscler Thromb Vasc Biol.2004;24:1266-1271.

16. Urbich C, Kuehbacher A, Dimmeler S. Role of microRNAs in vascular diseases, inflammation, and angiogenesis. Cardiovasc Res 2008;79:581-588.
17. Muhonen $\mathrm{P}$, Holthofer $\mathrm{H}$. Epigenetic and microRNA-mediated regulation in diabetes. Nephrol Dial Transplant 2009;24:1088-1096.

18. Cordes KR, Srivastava D. MicroRNA regulation of cardiovascular development. Circ Res 2009;104:724-732.

19. Burns WC, Twigg SM, Forbes JM, Pete J, Tikellis C, Thallas-Bonke V, et al. Connective tissue growth factor plays an important role in advanced glycation end product-induced tubular epithelialto- mesenchymal transition: implications for diabetic renal disease. J Am Soc Nephrol. 2006;17:2484-94.

20. Wang B, Koh P, Winbanks C, Coughlan MT, McClelland A, Watson A, et al. miR200a Prevents renal fibrogenesis through repression of TGF-beta2 expression. Diabetes. 2011;60:280-7.

21. Zhong $Q$, Kowluru RA. Regulation of matrix metalloproteinase-9 by epigenetic modifications and the development of diabetic retinopathy. Diabetes. 2013 Jul;62(7):2559-68.

22. Mazzola N. Review of Current and Emerging Therapies in Type 2 Diabetes Mellitus . Am J Manag Care. 2012 Jan;18(1 Suppl):S17-26.

23. Gray SG. The Potential of Epigenetic Compounds in Treating Diabetes. T. Tollefsbol (Ed): Epigenetics in Human Disease. 2012 Elsevier.

24. Nuria C. Bramswig and Klaus H. Kaestner. Epigenetics and diabetes treatment: an unrealized promise? Trends Endocrinol Metab. 2012 Jun;23(6):286-91.

25. Bolden JE, Peart MJ, Johnstone RW. Anticancer activities of histone deacetylase inhibitors. Nat Rev Drug Discov 2006;5:769e84.

26. Outeiro TF, Kontopoulos E, Altman S, Kufareva I, Strathearn KE, Amore AM, et al. Sirtuin 2 Inhibitors Rescue a-Synuclein-Mediated Toxicity in Models of Parkinson's Disease. Science 2007;317:516e9.

27. Schuetz A, Min J, Antoshenko $T$, Wang CL, Allali-Hassani A, Dong $A$, et al. Structural basis of inhibition of the human NADp-dependent deacetylase SIRT5 by suramin. Structure 2007;15:377e89.

28. Kiviranta PH, Leppanen J, Kyrylenko S, Salo HS, Lahtela-Kakkonen M, Tervo AJ, et al. N, N'-Bisbenzylidenebenzene- 1,4-diamines and N, N'Bisbenzylidenenaphthalene-1,4-diamines as Sirtuin Type 2 (SIRT2). Inhibitors. J Med Chem 2006;49:7907e11.

29. Tervo AJ, Suuronen T, Kyrylenko S, Kuusisto E, Kiviranta PH, Salminen A, et al. Discovering inhibitors of human sirtuin type 2: novel structural scaffolds. J Med Chem 2006;49:7239e41

30. Dashwood RH, Ho E. Dietary histone deacetylase inhibitors: From cells to mice to man. Semin Cancer Biol 2007;17:363e9.

31. Czech MP, Aouadi M, Tesz GJ. RNAi-based therapeutic strategies for metabolic disease. Nat Rev Endocrinol 2011.

Cet article a été publié dans le « Batna Journal of Medical Sciences » BJMS, l'organe officiel de "I'association de la Recherche Pharmaceutique - Batna »

Le contenu de la Revue est ouvert « Open Access » et permet au lecteur de télécharger, d'utiliser le contenu dans un but personnel ou d'enseignement, sans demander l'autorisation de l'éditeur/auteur.

Avantages à publier dans BJMS :

- Open access : une fois publié, votre article est disponible gratuitement au téléchargement

- Soumission gratuite : pas de frais de soumission, contrairement à la plupart des revues « Open Access »

- Possibilité de publier dans 3 langues : français, anglais, arabe

- Qualité de la relecture : des relecteurs/reviewers indépendants géographiquement, respectant l'anonymat, pour garantir la neutralité et la qualité des manuscrits.

Pour plus d'informations, contacter BatnaJMS@gmail.com

ou connectez-vous sur le site de la revue : www.batnajms.com

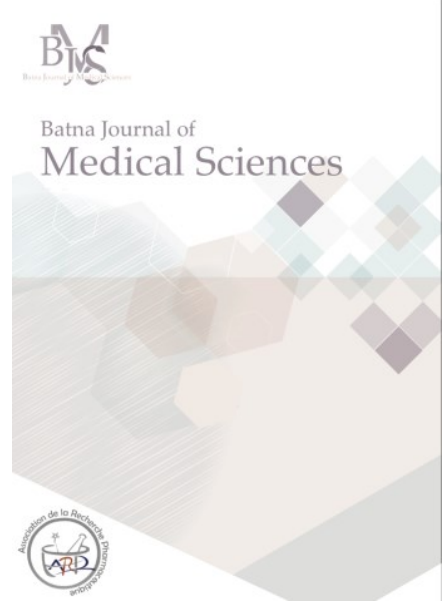




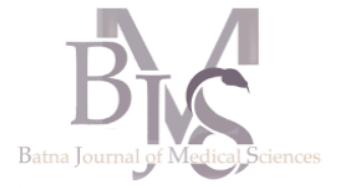
Centre AntiCancéreux Batna, Algérie

\section{Correspondance à :}

Adlen NEZZAR

nezzar.adlen@gmail.com

DOI :https://doi.org/10.48087/ B]MStf.2015.2114

\section{Il s'agit d'un article en libre} accès distribué selon les termes de la licence Creative Commons Attribution International License (CC BY 4.0), qui autorise une utilisation, une distribution et une reproduction sans restriction sur tout support ou format, à condition que l'auteur original et la revue soient dûment crédités.

\section{Biologie moléculaire et cancer de la thyroïde}

\section{Molecular biology and thyroid cancer}

\section{Adlen Nezzar}

\section{RÉSUMÉ}

Pendant les 20 dernières années, la compréhension de la biologie moléculaire des cancers de la thyroïde a progressé, le développement des analyses moléculaires laisse entrevoir des implications importantes pour une aide au diagnostic en pathologie thyroïdienne; ces analyses moléculaires peuvent aussi potentiellement permettre de mieux apprécier le pronostic de certaines lésions thyroïdiennes. Quatre types de mutation représentent la grande majorité des mutations somatiques actuellement connues, ayant le plus grand impact pour le diagnostic et le pronostic des carcinomes folliculaires et papillaires de la thyroïde : il s'agit des mutations ponctuelles de BRAF et de RAS et des réarrangements de RET/PTC et de PAX8/ PPAR $\gamma$. Les mutations constitutionnelles de RET permettent de distinguer les formes familiales et les formes sporadiques des carcinomes médullaires de la thyroïde. La mutation activatrice $\mathrm{V} 600 \mathrm{E}$ du gène BRAF est l'évènement oncogénique le plus fréquent et le plus spécifique des carcinomes papillaires (CP). Les miARN devraient rapidement avoir un intérêt diagnostique des tumeurs folliculaires sur cytoponction, grâce aux travaux récents explorant un plus grand nombre de classes tumorales et notamment des groupes de tumeurs histologiquement atypiques.

Mots-clés : biologie moléculaire, génétique, cancer de la thyroïde, mutation, micro-ARN.

\begin{abstract}
During the last two decades, the understanding of molecular biology of thyroid cancers has greatly improved, the development of molecular biology analyses in thyroid pathology is linked to interesting new tools for diagnosis improvement; these molecular biology analyses can also potentially allow to better estimate the prognosis of certain thyroid tumors. Four types of mutation represent the main somatic mutations currently known which can have a diagnostic and a prognostic impact in vesicular and papillary thyroid carcinoma: BRAF and RAS mutations and RET/PTC and PAX8/PPAR $\gamma$ rearrangements. Additionally, RET mutations distinguish the familial and sporadic forms of medullary thyroid carcinoma. The activating mutation of the BRAF gene, V600E, is the most common and specific genetic alteration in PTC. Measurement of several miRNA should have soon a diagnostic interest for follicular tumors according to recent studies exploring atypical tumors on histology.
\end{abstract}

Keywords: molecular biology, genetics, thyroid cancer, mutation, micro-RNA

\section{Pour citer l'article :}

Nezzar A. Biologie moléculaire et cancer de la thyroïde. Batna J Med Sci 2015;2(1):60-65.

https://doi.org/10.48087/ BJMStf.2015.2114

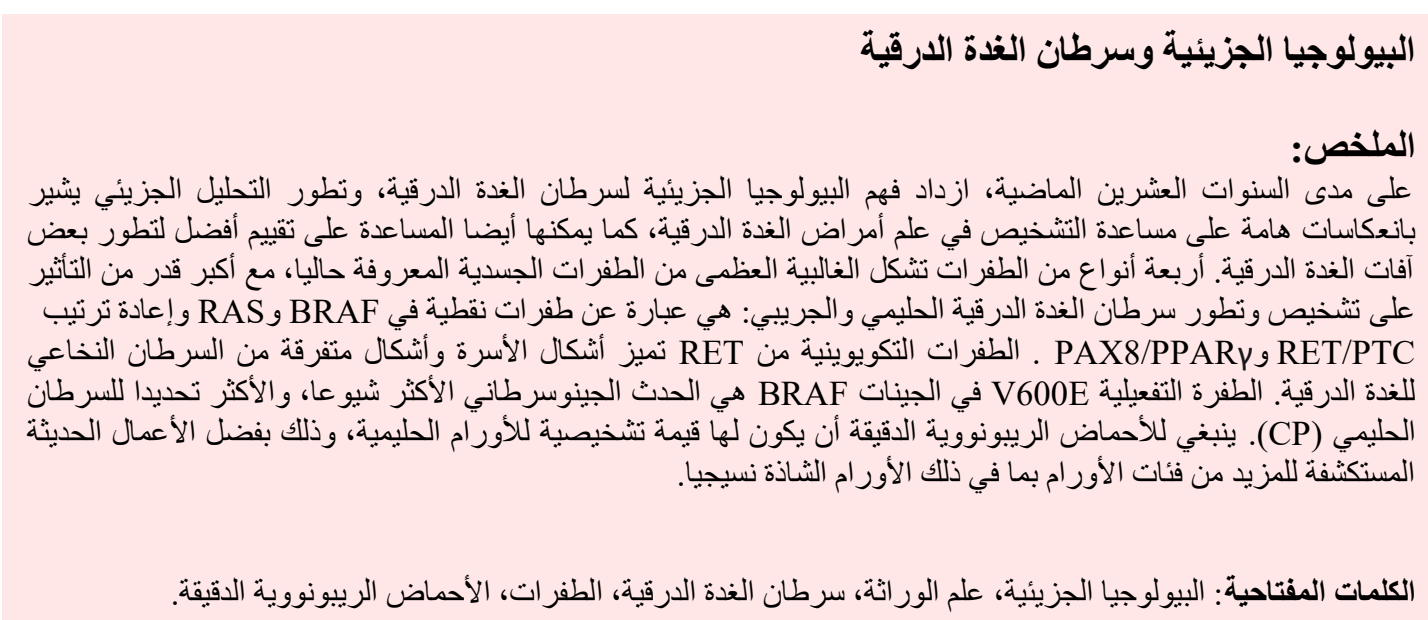

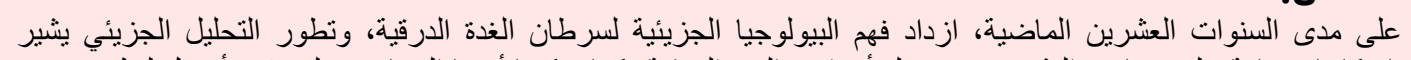

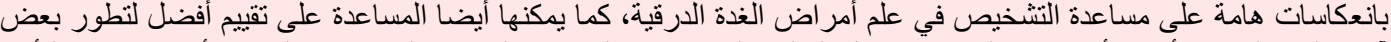

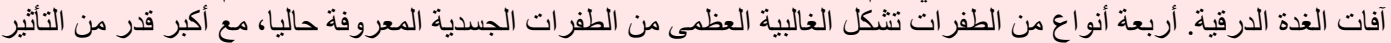

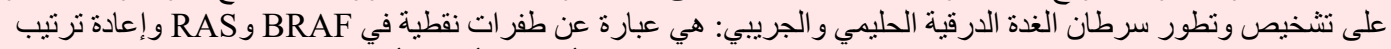
PAX8/PPARy RET/PTC

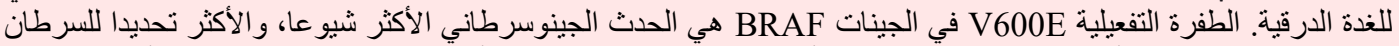

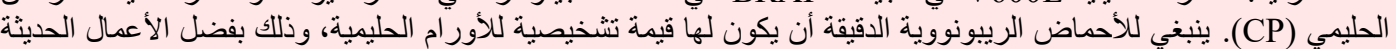
المستكثفة للمزيد من فئات الأورام بما في ذللك الأورام الثاذة نسيجيا.

الكلمات المفتاحية: البيولوجيا الجزيئية، علم الور اثة، سرطان الغدة الدرقية، الطفرات، الأحماض الريبونووية الدقيقة. 


\section{INTRODUCTION}

L'incidence des cancers de la thyroïde est en constante augmentation [1]. Cette augmentation est due, au moins en partie, aux possibilités de dépistage des lésions thyroïdiennes par une imagerie de plus en plus précise et aux indications de plus en plus fréquentes des cytoponctions thyroïdiennes. Les altérations génétiques et les différentes mutations observées en pathologie thyroïdienne sont nombreuses, variant selon le sous-type histologique. Les cancers de souche folliculaire sont d'histologie papillaire (75-80\%), folliculaire (5-10\%) et indifférenciée (2-5\%), alors que les cancers médullaires qui sont beaucoup plus rares (5-10\%) et dérivent des cellules C. Les mécanismes de tumorigenèse peuvent donc être associés à des changements qualitatifs (présence de mutation) ou quantitatifs (niveau d'expression des gènes). Les anomalies génétiques liées à la tumorigenèse thyroïdienne impliquent les deux voies de transduction du signal que sont la voie MAPkinase et la voie PI3kinase/Akt. L'activation de la voie PI3kinase/Akt est associée au développement de tumeurs différenciées de mauvais pronostic ou aux tumeurs anaplasiques [4,5]. L'activation de la voie MAPkinase est le plus souvent associée au développement des cancers papillaires (CPT). Parmi les mutations somatiques retrouvées dans les CPT, les mutations dans les gènes BRAF sont les plus fréquentes. Elles représentent 40 à $45 \%$ des anomalies rencontrées selon les séries alors que le réarrangement Ret/PTC et les mutations dans les gènes RAS sont présentes dans 10 à 20\% des CPT [6] . La majorité des cancers folliculaires et papillaires est guérie après traitement et leur survie à 20 ans est supérieure à 90\% [2]. Les métastases à distance surviennent chez moins de $10 \%$ des patients, et la rémission complète est obtenue avec les traitements par l'iode 131 chez un tiers des patients métastatiques [3]. Les formes réfractaires de cancer de la thyroïde sont peu fréquentes [2]. La maladie métastatique est souvent lentement évolutive, de nouvelles molécules sont utilisées dans le cancer de la thyroïde, et leur utilisation repose sur la biologie de ces cancers.

\section{ALTÉRATIONS GÉNÉTIQUES MOLÉCULAIRES EN PATHOLOGIE THYROÏDIENNE}

\section{La mutation de BRAF}

Les mutations activatrices sur le gène BRAF sont les altérations génétiques les plus communes des carcinomes papillaires de la thyroïde (CPT), trouvées dans environ 45\% de ces tumeurs selon les séries [7-10]. Les anomalies du gène BRAF sont très majoritairement situées sur le nucléotide 1799 et résultent de la substitution d'une valine par une glutamine au niveau du résidu 600 (V600E). Cette mutation ponctuelle entraîne une activation constitutive de la kinase, associée à son pouvoir oncogénique [11].

D'autres mutations du gène BRAF sont plus exceptionnellement retrouvées, en particulier dans certains CPT associés à des antécédents d'exposition à des radiations ionisantes ou dans des types histologiques rares. La mutation BRAFV600 est essentiellement observée dans les CPT de type histologique « conventionnel ou classique » $[12,13]$ ainsi que dans la variante à cellules hautes [14], bien plus rarement dans la variante folliculaire des CPT $[15,16]$. Cette mutation peut parfois être observée dans les carcinomes anaplasiques et dans certains carcinomes peu différenciés se développant sur un CPT [10]. Elle n'est jamais retrouvée dans les carcinomes folliculaires ou dans les tumeurs bénignes de la thyroïde, ce qui est en fait un marqueur spécifique des CPT [7, $10]$.

\section{Le réarrangement de RET/PTC}

Le réarrangement de RET/PTC est l'autre altération génétique également trouvée dans les CPT. Ce réarrangement est formé par la fusion entre la portion 3' du récepteur tyrosine kinase RET et la portion 5' de plusieurs autres gènes. Le réarrangement de RET/PTC est détecté dans environ 20\% des cas sporadiques de CPT de l'adulte [17]. RET/PTC survient aussi avec une plus forte incidence en cas d'antécédent d'irradiation (50-80\%) et en cas de CPT survenant chez l'enfant et l'adulte jeune (40-70\%). De façon globale, les CPT positifs pour RET/PTC surviennent préférentiellement chez des sujets plus jeunes, ont une architecture histologique papillaire classique et une tendance à être associés à des métastases ganglionnaires

\section{La mutation de RAS}

Les mutations ponctuelles du gène RAS ne sont pas spécifiques d'un type particulier de tumeur de la thyroïde et sont ainsi observées dans les CPT, les carcinomes et les adénomes folliculaires. La résultante de ces mutations est que la protéine mutée devient activée de façon permanente et stimule de façon chronique les cibles situées en aval. Dans les CPT, les mutations de RAS surviennent dans 10-20\% des tumeurs. Il est intéressant de constater que parmi les CPT présentant une mutation de RAS, il s'agit presque toujours de CPT à variante histologique folliculaire, encapsulés, et avec peu ou pas de métastases ganglionnaires. Finalement, les mutations de RAS sont retrouvées dans 40-50\% des carcinomes folliculaires et dans $20-30 \%$ des adénomes folliculaires.

\section{Le réarrangement PAX8/PPAR $\gamma$}

Le réarrangement de PAX8/PPAR $\gamma$ résulte d'une translocation $\mathrm{t}(2 ; 3)(\mathrm{q} 13 ; \mathrm{p} 25)$, entraînant la fusion entre le gène PAX8 et le gène du récepteur PPAR $\gamma$ (" peroxisome proliferator activated receptor ») [18]. Ce réarrangement conduit à une forte expression de la protéine PPAR $\gamma$, mais les mécanismes de cette transformation cellulaire consécutifs à cet événement génétique ne sont pas encore totalement élucidés. PAX8/PPAR $\gamma$ est mis en évidence dans 30-40\% des formes histologiques " classiques 》 des carcinomes folliculaires, et avec une plus faible prévalence dans les carcinomes oncocytaires [10]. Les tumeurs présentant ce réarrangement ont tendance à se développer chez les sujets plus jeunes, d'être de plus petite taille, d'avoir une architecture solide et d'être angio-invasives. Plus rarement, ce réarrangement peut être observé dans les adénomes folliculaires (2-10\%) et dans des CPT de variante folliculaire [19]. Cependant, il a été décrit que les adénomes folliculaires possédant ce réarrangement avaient typiquement une capsule épaisse et montraient un profil immunohistochimique plus " en faveur " d'un carcinome, laissant ainsi suggérer pour certains auteurs que ces tumeurs représenteraient finalement des carcinomes folliculaires in situ ou pré-invasifs ou bien des tumeurs malignes pour lesquelles l'invasion avait été sous-estimée lors de l'analyse histologique initiale [20].

\section{Les mutations de RET et les carcinomes médullaires de la thyroïde}

Les carcinomes médullaires de la thyroïde (CMT) sont des tumeurs rares. Ils sont plus agressifs que les carcinomes bien différenciés d'origine folliculaire, avec un taux de survie 
moyen de $50 \%$ à 10 ans, responsables d'environ $13 \%$ des décès liés aux carcinomes thyroïdiens [21]. Il n'y a pas eu d'amélioration de la survie des patients sur ces 30 dernières années [22], imposant la nécessité de développer de nouvelles stratégies thérapeutiques. L'origine des CMT est la cellule C (ou cellule para-folliculaire). Ils peuvent sécréter de la calcitonine, ou d'autres hormones comme l'ACTH. On distingue les CMT sporadiques (75\% des cas environ) et les CMT familiaux $(25 \%$ des cas) héréditaires à transmission autosomique dominante. Parmi ces derniers, on distingue une forme de CMT familial isolée sans polyendocrinopathie et une forme associée à une néoplasie endocrinienne multiple (NEM) de type IIa ou IIb. Le diagnostic de formes familiales ou sporadiques des CMT passe par une détection des mutations germinales de RET effectuées sur un prélèvement sanguin $[22,23]$.

\section{Les microARN}

De très courts segments d'ARN, appelés microARN, considérés pendant longtemps comme des produits de dégradation métabolique sans activité physiologique, font actuellement l'objet de nombreuses recherches et publications [24, 25, 26, 27]. Les miRNA sont des petits ARN simple brin non-codant, constitués de 21 à 23 nucléotides, qui s'associent aux protéines de la famille Argonaute afin de contrôler l'expression des gènes [28]. Dans le génome humain, il existe plusieurs centaines de miRNA impliqués dans le contrôle positif ou négatif de la prolifération cellulaire [29]. Puisqu'on estime qu'ils réguleraient environ un tiers des gènes codant pour des protéines, leur implication dans le développement tumoral est liée soit à une modification du nombre de copies de miRNA susceptibles d'agir sur des cibles ARNm, correspondant à des gènes suppresseurs de tumeurs ou des oncogènes, soit à des modifications de leur affinité pour les cibles ARNm en présence de polymorphismes (SNP ou single nucleotide polymorphism ) dans la région d'appariement du miRNA avec les ARNm cibles. Ainsi, un appariement anormal entre le miRNA et l'ARNm peut être lié à la présence de polymorphismes dans l'ARNm du gène c-KIT, impliqué dans les voies de régulation du cycle cellulaire, et sont spécifiquement associés au risque de développement des cancers papillaires de la thyroïde [30]. Plusieurs microARN, incluant mir-146b, mir-221, mir-222, mir-181b, mir-155 et mir-224, sont surexprimés de façon très significative dans les CPT [31,32]. De façon intéressante les niveaux de surexpression de certains de ces microARN peuvent être corrélés avec le profil mutationnel de certains CPT.

\section{Le vascular endothelial growth factor (VEGF)}

Plusieurs facteurs sont impliqués dans le développement de la vascularisation de la tumeur primitive et des métastases, dont le vascular endothelial growth factor (VEGF) est le chef de file [33,34]. L'activité angiogénique du VEGF s'exerce au niveau des cellules endothéliales des vaisseaux après liaison à 2 récepteurs membranaires à activité tyrosine kinase, VEGFR1 (Flt-1) et VEGFR-2 (Flk-1/KDR), qui activent la voie des MAP-kinases. L'angiogenèse tumorale permet aux cellules tumorales de survivre et de proliférer. Dans le cancer de la thyroïde, la densité de micro-vaisseaux est plus élevée que dans le tissu thyroïdien normal ; l'expression de VEFG est élevée et est corrélée à une extension plus importante de la tumeur de la thyroïde, à un taux élevé de métastases et de récidives et à une diminution de la survie sans récidive et donc le ciblage du VEGF inhibe la croissance de la tumeur de la thyroïde $[35,36]$.

\section{IMPACT DES MARQUEURS MOLÉCULAIRES EN PATHOLOGIE THYROÏDIENNE}

La détection des différents marqueurs moléculaires décrits plus hauts peut se faire dans les produits cytologiques issus des ponctions thyroïdiennes à l'aiguille fine, ou bien à partir des pièces de résection chirurgicale [37]. Les résultats obtenus offrent des informations utiles et additionnelles pour le diagnostic, pour la prise en charge thérapeutique et pour le suivi des patients ayant un nodule thyroïdien $[38,39]$.

\section{Intérêt des tests moléculaires développés à partir des cytoponctions thyroïdiennes à l'aiguille fine}

L'analyse cytologique des prélèvements sur ponction à l'aiguille fine des nodules thyroïdiens est une approche quasi incontournable pour définir le diagnostic de ces lésions et pour adopter une stratégie thérapeutique. L'approche moléculaire augmente de façon significative la spécificité du diagnostic. Il semble que l'impact diagnostique de ces recherches est d'autant plus important qu'un panel de mutations est recherché (BRAF, RAS, Ret/PTC, PPARg /Pax8), en particulier pour les lésions de classes indéterminées en cytologie [40]. Cette dernière catégorie englobe en fait plusieurs sous-types de lésions thyroïdiennes : les lésions folliculaires de signification indéterminée, les néoplasies folliculaires et les néoplasies à cellules de Hurthle, et les lésions suspectes de malignité, corrélées respectivement avec un risque estimée pour la malignité de $5-10 \%, 20-30 \%$ et 50 $75 \%$ [41]. Compte tenu de l'absence de diagnostic de certitude, la grande majorité de ces patients sera donc opérée, mais seulement 8-17\% des nodules correspondant se révéleront en fait comme étant des tumeurs malignes. Un nombre croissant de publications montre que les différentes analyses moléculaires pratiquées à partir de produits de cytoponction thyroïdienne augmentent de façon significative la spécificité du diagnostic des nodules thyroïdiens ainsi ponctionnés $[42,43]$. Parmi ces publications, une grande majorité explore le rôle diagnostique de la présence d'une mutation BRAF [44-47]. Toutes les études réalisées montrent que parmi les cytoponctions qui étaient BRAF positives, les tumeurs enlevées chirurgicalement étaient des CPT. Il faut souligner qu'un grand nombre des analyses moléculaires, réalisées à la recherche de la mutation de BRAF est positive pour cette mutation, avait un diagnostic cytologique de lésions indéterminées ou bien étaient non contributives. Un certain nombre de diagnostics cytologiques classant des lésions comme étant bénignes, mais mutées pour BRAF, correspondait à un CPT à l'examen histologique. Pour certains auteurs, la présence de la mutation BRAF devrait même conduire à une thyroïdectomie totale, sans tenir compte de l'aspect bénin observé cytologiquement.

\section{Intérêt des tests moléculaires développés sur pièces opératoires}

Les tumeurs portant la mutation de BRAF correspondent histologiquement à des CPT classiques et à des CPT à cellules hautes, ne devant pas ainsi poser de problème de diagnostic. Ainsi, la présence de cette mutation n'apporte pas d'aide supplémentaire au diagnostic de ces lésions. Les variantes folliculaires des CPT sont souvent associées à une mutation de RAS, mais cette mutation peut être aussi observée dans les adénomes folliculaires, ce qui limite l'intérêt de cette analyse moléculaire. Lorsqu'une lésion folliculaire est suspecte d'être un carcinome, la détection de PAX8/PPAR $\gamma$ peut être d'une grande aide au diagnostic. Une étude a été réalisée dans le but de voir si les analyses moléculaires pouvaient aider au 
diagnostic des tumeurs thyroïdiennes de potentiel de malignité incertain [48]. Cependant même si un certain nombre de mutations ponctuelles a été retrouvé, notamment sur le gène RAS, il n'existait pas dans cette série de profil mutationnel particulier pouvant orienter le diagnostic vers l'une des entités constituant le groupe de ces tumeurs [48]. Ainsi, la recherche de ces mutations semble avoir un impact limité dans le cadre du diagnostic des lésions histologiques thyroïdiennes.

\section{PATHOLOGIE MOLÉCULAIRE DE LA THYROÏDE ET FACTEURS PRÉDICTIFS D'AGRESSIVITÉ TUMORALE}

La plupart des cancers bien différenciés de la thyroïde, en particulier ceux de petite taille et bien localisés, ont un comportement peu agressif et peuvent être parfaitement traités. Cependant, certains de ces cancers ont de façon non prévisible un potentiel évolutif plus important et nécessiteraient de ce fait un traitement d'emblée plus «agressif». Les biomarqueurs moléculaires peuvent ainsi contribuer à évaluer le pronostic de ces tumeurs [49].

\section{BRAF}

La mutation de BRAF est responsable d'une altération de la fonction du transporteur d'iode, expliquant probablement certains échecs thérapeutiques [50]. BRAF favorise aussi l'évolution vers une dédifférenciation tumorale et vers le développement possible d'un carcinome anaplasique, dont le pronostic est très défavorable. Près de $45 \%$ des tumeurs BRAF positives sont significativement associées à un stade tumoral avancé avec un risque significativement plus élevé de métastases locorégionales et à distance, contrairement aux tumeurs BRAF négatives. La valeur pronostique de cette mutation semble être aussi particulièrement importante pour les microcarcinomes papillaires. Si la plupart de ces lésions ne sont pas accompagnées de récidive, un certain nombre d'entre elles peuvent entraîner des métastases et le décès des patients. Ainsi, plusieurs études montrent que les microcarcinomes papillaires présentant une mutation de BRAF ont un potentiel métastatique ganglionnaire plus important et un potentiel invasif extra-thyroïdien [51,52]. Ces microcarcinomes papillaires à potentiel agressif devraient donc certainement bénéficier d'un traitement initialement plus agressif. Il paraît important d'essayer de déterminer dans le futur le groupe de CPT BRAF mutés à fort potentiel agressif. En effet, tous les CPT BRAF mutés ne sont pas agressifs et les CPT non mutés pour BRAF peuvent aussi évoluer d'une façon péjorative. Enfin, les tumeurs BRAF positives se développent à un âge significativement plus tardif (49,3 - 16,2 ans) que celles BRAF négatives (35,0 - 17,3 ans) [53].

\section{RAS}

Le rôle de RAS comme biomarqueur pronostique des tumeurs thyroïdes n'est pas déterminé. Comme cette mutation est également retrouvée dans des tumeurs folliculaires bénignes, le statut RAS par lui-même ne peut pas être utilisé pour évaluer le pronostic tumoral. Quelques études montrent toutefois que la présence de RAS détectée dans certains CPT ou dans des carcinomes folliculaires est corrélée à un pronostic plus défavorable, avec en particulier le développement de métastases osseuses [54].

\section{RET/PTC}

La corrélation entre la présence de RET/PTC et le pronostic des CPT est incertaine. Des études montrent une corrélation avec un pronostic plus favorable. Ainsi, contrairement aux CPT ayant une mutation de BRAF et de NRAS, ceux ayant la mutation de RET/PTC, en particulier de RET/PTC1, ont une plus faible probabilité de progression vers un carcinome peu différencié ou vers un carcinome anaplasique. Cependant, en cas de mutations de BRAF et de RET/PTC, certains CPT auraient tendance à récidiver plus fréquemment [55].

\section{MiRNA}

Plus fréquemment, le profil d'expression des miRNA va être modifié selon la classe de tumeurs et/ou l'agressivité tumorale et ainsi servir de biomarqueurs diagnostiques ou pronostiques. Dans le cas des cancers thyroïdiens, une expression différentielle de miRNA a été décrite dans plusieurs études rassemblant près de 500 échantillons de tumeurs dont plus de $40 \%$ sont des CPT [56, 57]. Cinq de ces études ont servi de référence pour identifier les profils différentiels des cancers folliculaires, papillaires, des cancers peu différenciés et des anaplasiques [58]. Elles montrent que les marqueurs miRNA sont majoritairement surexprimés dans les tumeurs différenciées, alors qu'ils sont sousexprimés dans les tumeurs dédifférenciées.

\section{PERSPECTIVES}

Les options thérapeutiques des patients ayant des cancers de la thyroïde réfractaires sont élargies avec l'avènement des thérapies ciblées. Cependant, plusieurs points méritent d'être soulignés : ces traitements procurent un bénéfice clinique, somme des réponses complètes, des réponses partielles et des stabilisations tumorales, ainsi que par l'amélioration de la survie sans progression. Dans tous les cas, il est important de prendre en compte les critères de progression avant l'inclusion pour interpréter la stabilité. Il faut noter que les réponses tumorales ont été partielles, et souvent transitoires, ce qui incite à poursuivre les essais avec de nouvelles molécules utilisées soit seules, soit en association. La cible biologique impliquée dans la réponse à chaque traitement reste mal déterminée, de même que les mécanismes de résistance primaire ou secondaire. À l'avenir, ceci permettra de définir pour chaque patient le traitement le mieux adapté. De nouvelles possibilités thérapeutiques sont ouvertes par les données expérimentales, par exemple l'inhibition de MEK en cas de mutation BRAF [59]. L'utilisation des marqueurs pour documenter la réponse n'a pas été considérée comme critère de jugement dans la majorité des études. Enfin, il est nécessaire d'évaluer la toxicité de ces traitements et la qualité de vie des patients à court et à long terme.

\section{CONCLUSION}

Les examens de biologie moléculaire doivent permettre dans le futur d'améliorer le diagnostic des lésions thyroïdiennes. Le typage moléculaire des tumeurs thyroïdiennes sur prélèvement de ponction à l'aiguille fine est très prometteur dans l'identification de facteurs diagnostiques et pronostiques notamment de certains microcarcinomes papillaires à potentiel agressif. Pour l'instant, seule la recherche combinée de la mutation BRAF et la mesure du taux de miR-146b pourraient permettre de détecter les CPT de mauvais pronostic et d'identifier ceux pouvant bénéficier d'une meilleure prise en charge thérapeutique.

Déclaration d'intérêts : l'auteur ne déclare aucun conflit d'intérêt en rapport avec cet article. 


\section{RÉFÉRENCES}

1. Davies L, Welch HG. Increasing incidence of thyroid cancer in the United States, 1973-2002. JAMA 2006;295(18):2164-7.

2. Schlumberger MJ. Papillary and follicular thyroid carcinoma. N Engl J Med 1998;338:297-306.

3. Durante C, Haddy N, Baudin E, et al. Long term outcome of 444 patients with distant metastases from papillary and follicular thyroid carcinoma : benefits and limits of radioiodine therapy. J Clin Endocrinol Metab 2006;91:2892-9.

4. Kundra P, Burman KD. Thyroid cancer molecular signaling pathways and use of targeted therapy. Endocrinol Metab Clin North Am 2007;36:839-53. viii.

5. Liu Z, Hou P, Ji M, et al. Highly prevalent genetic alterations in receptor tyrosine kinases and phosphatidylinositol 3-kinase/akt and mitogen-activated protein kinase pathways in anaplastic and follicular thyroid cancers. J Clin Endocrinol Metab 2008;93:3106-16.

6. Lassalle $\mathrm{S}$, Hofman $\mathrm{V}$, Ilie $\mathrm{M}$, et al. Clinical impact of the detection of BRAF mutations in thyroid pathology: potential usefulness as diagnostic, prognostic and theragnostic applications. Curr Med Chem 2010;17:1839-50.

7. Lassalle $\mathrm{S}$, Hofman $\mathrm{V}$, Ilie $\mathrm{M}$, et al. Clinical impact of the detection of BRAF mutations in thyroid pathology: potential usefulness as diagnostic, prognostic and theragnostic applications. Curr Med Chem 2010;17(17):1839-50.

8. Cohen $\mathrm{Y}$, Xing $\mathrm{M}$, Mambo $\mathrm{E}$, et al. BRAF mutation in papillary thyroid carcinoma. J Natl Cancer Inst 2003;95(8):625-7.

9. Kebebew E, Weng J, Bauer J, et al. The prevalence and prognostic value of BRAF mutation in thyroid cancer. Ann Surg 2007;246(3):466- 70.

10. Xing M. BRAF mutation in thyroid cancer. Endocr Relat Cancer 2005;12(2):24562

11. Fukushima $T$, Suzuki $S$, Mashiko $M$, et al. BRAF mutations in papillary carcinomas of the thyroid. Oncogene 2003;22(41):6455-7.

12. Trovisco V, Vieira de Castro I, Soares $P$ et al. BRAF mutations are associated with some histological types of papillary thyroid carcinoma. J Pathol 2004;202(2):247-51

13. Trovisco $V$, Soares $P$, Sobrinho-Simões $M$. B-RAF mutations in the etiopathogenesis, diagnosis, and prognosis of thyroid carcinomas. Hum Pathol 2006;37(7):781-6

14. LiVolsi VA. Papillary carcinoma tall cell variant (TCV): a review. Endocr Pathol 2010;21(1):12-5.

15. Jakubowski M, Hunt JL. BRAF mutational analysis in papillary carcinomas with mixed follicular and papillary growth patterns. Am J Surg Pathol 2009;33(11):1590-3.

16. Rivera M, Ricarte-Filho J, Knauf J, et al. Molecular genotyping of papillary thyroid carcinoma follicular variant according to its histological subtypes (encapsulated vs infiltrative) reveals distinct BRAF and RAS mutation patterns. Mod Pathol 2010

17. De Groot JW, Links TP, Plukker JT, et al. RET as a diagnostic and therapeutic target in sporadic and hereditary endocrine tumors. Endocr Rev 2006;27(3):535-60.

18. Kroll TG, Sarraf P, Pecciarini L, et al. PAX8-PPARgamma1fusion oncogene in human thyroid carcinoma [corrected]. Science 2000;289(5483):1357-60.

19. Castro $P$, Rebocho AP, Soares RJ, et al. PAX8-PPARgamma rearrangement is frequently detected in the follicular variant of papillary thyroid carcinoma. J Clin Endocrinol Metab 2006;91(1):213-20.

20. Nikiforova MN, Lynch RA, Biddinger PW, et al. RAS point mutations and PAX8PPAR gamma rearrangement in thyroid tumors: evidence for distinct molecular pathways in thyroid follicular carcinoma. J Clin Endocrinol Metab 2003;88(5):2318-26

21. Kebebew E, Clark OH. Medullary thyroid cancer. Curr Treat Options Oncol 2000;1(4):359-67.

22. Roman S, Mehta P, Sosa JA. Medullary thyroid cancer: early detection and novel treatments. Curr Opin Oncol 2009;21(1):5-10.

23. Cakir M, Grossman AB. Medullary thyroid cancer: molecular biology and novel molecular therapies. Neuroendocrinology 2009;90(4):323-48.

24. Croce $\mathrm{CM}$. Causes and consequences of microRNA dysregulation in cancer. Nat Rev Genet 2009;10(10):704-14.

25. Garzon R, Calin GA, Croce CM. .MicroRNAs in Cancer. Annu Rev Med 2009;60:167-79.
26. Iorio $\mathrm{MV}$, Croce $\mathrm{CM}$. MicroRNAs in cancer: small molecules with a huge impact. J Clin Oncol 2009;27(34):5848-56.

27. Ortholan $\mathrm{C}$, Puissegur MP, llie M, et al. MicroRNAs and lung cancer: new oncogenes and tumor suppressors, new prognostic factors and potential therapeutic targets. Curr Med Chem 2009;16(9):1047-61.

28. Kim VN, Han J, Siomi MC. Biogenesis of small RNAs in animals. Nat Rev Mol Cell Biol 2009;10:126-39.

29. Lewis BP, Burge CB, Bartel DP. Conserved seed pairing, often flanked by adenosines, indicates that thousands of human genes are microRNA targets. Cell 2005;120:15-20.

30. He H, Jazdzewski K, Li W, Liyanarachchi S, Nagy R, Volinia S, et al. The role of microRNA genes in papillary thyroid carcinoma. Proc Natl Acad Sci U S A 2005;102:19075-80.

31. Nikiforova MN, Tseng GC, Steward D, et al. MicroRNA expression profiling of thyroid tumors: biological significance and diagnostic utility. J Clin Endocrinol Metab 2008;93(5):1600-8.

32. Nikiforova MN, Chiosea SI, Nikiforov YE. MicroRNA expression profiles in thyroid tumors. Endocr Pathol 2009;20(2):85-91.

33. Bunone $G$, Vigneri $P$, Mariani $L$, et al. Expression of angiogenesis stimulators and inhibitors in human thyroid tumors and correlation with clinical pathological features. Am J Pathol 1999;155:1967-76.

34. Fenton C, Patel A, Dinauer C, et al. The expression of vascular endothelial growth factor and the type 1 vascular endothelial growth factor receptor correlate with the size of papillary thyroid carcinoma in children and young adults. Thyroid 2000;10:349-57.

35. Bauer AJ, Patel A, Terrell R, et al. Systemic administration of vascular endothelial growth factor monoclonal antibody reduces the growth of papillary thyroid carcinoma in a nude mouse model. Ann Clin Lab Sci 2003:33:192-9.

36. Bauer AJ, Terrell R, Doniparthi NK, et al. Vascular endothelial growth factor monoclonal antibody inhibits growth of anaplastic thyroid cancer xenografts in nude mice. Thyroid 2002;12:953-61.

37. Cohen $Y$, Rosenbaum $E$, Clark DP, et al. Mutational analysis of BRAF in fine needle aspiration biopsies of the thyroid: a potential application for the preoperative assessment of thyroid nodules. Clin Cancer Res 2004;10(8):27615 .

38. Pinto $A E$, Leite $V$, Soares J. Clinical implications of molecular markers in follicular cell-derived thyroid cancer. Expert Rev Mol Diagn 2009;9(7):679-94.

39. Wreesmann VB, Singh B. Clinical impact of molecular analysis on thyroid cancer management. Surg Oncol Clin N Am 2008;17(1):1-35.

40. Nikiforov YE, Nikiforova MN. Molecular genetics and diagnosis of thyroid cancer. Nat Rev Endocrinol 2011;7:569-80.

41. morphologic criteria for cytologic diagnosis of thyroid lesions: a synopsis of the National Cancer Institute Thyroid Fine-Needle Aspiration State of the Science Conference. Diagn Cytopathol 2008;36(6):425-37.

42. French CA, Fletcher JA, Cibas ES, et al. Molecular detection of PPAR gamma rearrangements and thyroid carcinoma in preoperative fine-needle aspiration biopsies. Endocr Pathol 2008;19(3):166-74.

43. Gómez Saez JM. Diagnostic usefulness of tumor markers in the thyroid cytological samples extracted by fine-needle aspiration biopsy. Endocr Metab Immune Disord Drug Targets 2010;10(1):47-56.

44. Guo F, Hou P, Shi B. Detection of BRAF mutation on fine needle aspiration biopsy specimens: diagnostic and clinical implications for papillary thyroid cancer. Acta Cytol 2010;54(3):291-5.

45. Jin L, Sebo TJ, Nakamura $\mathrm{N}$, et al. BRAF mutation analysis in fine needle aspiration (FNA) cytology of the thyroid. Diagn Mol Pathol 2006;15(3):136-43.

46. Marchetti I, Lessi F, Mazzanti CM, et al. A morpho-molecular diagnosis of papillary thyroid carcinoma: BRAF V600E detection as an important tool in preoperative evaluation of fine-needle aspirates. Thyroid 2009;19(8):837-42.

47. Nam SY, Han BK, Ko EY, et al. BRAF V600E mutation analysis of thyroid nodules needle aspirates in relation to their ultrasongraphic classification: a potential guide for selection of samples for molecular analysis. Thyroid 2010;20(3):273-9.

48. Hofman V, Lassalle S, Bonnetaud C, et al. Thyroid tumours of uncertain malignant potential: frequency and diagnostic reproducibility. Virchows Arch 2009;455(1):21-33.

49. Handkiewicz-Junak D, Czarniecka A, Jarzab B. Molecular prognostic markers in papillary and follicular thyroid cancer: Current status and future directions. Mol Cell Endocrinol 2010;322(1-2):8-28. 


\section{DOSSIER THÉMATIQUE}

50. Elisei R, Ugolini C, Viola D, et al. BRAF(V600E) mutation and outcome of patients with papillary thyroid carcinoma: a 15-year median follow-up study. J Clin Endocrinol Metab 2008;93(10):3943-9.

51. Lee $X$, Gao M, Ji Y, Yu Y, et al. Analysis of differential BRAF(V600E) mutational status in high aggressive papillary thyroid microcarcinoma. Ann Surg Oncol 2009;16(2):240-5.

52. Lupi C, Giannini R, Ugolini C, et al. Association of BRAF V600E mutation with poor clinicopathological outcomes in 500 consecutive cases of papillary thyroid carcinoma. J Clin Endocrinol Metab 2007;92(11):4085-90.

53. Nikiforova MN, Kimura ET, Gandhi M, et al. BRAF mutations in thyroid tumors are restricted to papillary carcinomas and anaplastic or poorly differentiated carcinomas arising from papillary carcinomas. J Clin Endocrinol Metab 2003; 88:5399-404.

54. Garcia-Rostan G, Zhao H, Camp RL, et al. ras mutations are associated with aggressive tumor phenotypes and poor prognosis in thyroid cancer. J Clin Oncol 2003;21(17):3226-35.
55. Henderson YC, Shellenberger TD, Williams MD, et al. High rate of BRAF and RET/PTC dual mutations associated with recurrent papillary thyroid carcinoma. Clin Cancer Res 2009;15(2):485-91

56. Braun J, Hoang-Vu C, Dralle H, Huttelmaier S. Downregulation of microRNAs directs the EMT and invasive potential of anaplastic thyroid carcinomas. Oncogene 2010;29:4237-44.

57. Weber F, Teresi RE, Broelsch CE, Frilling A, Eng C. A limited set of human MicroRNA is deregulated in follicular thyroid carcinoma. J Clin Endocrinol Metab 2006;91:3584-91.

58. Braun J, Huttelmaier S. Pathogenic mechanisms of deregulated micro-RNA expression in thyroid carcinomas of follicular origin. Thyroid Res 2001;4(Suppl. 1):S1.

59. Solit DB, Garraway LA, Pratilas CA, Sawai A, Getz G, Basso A et al. BRAF mutation predicts sensitivity to MEK inhibition. Nature 2006;439:358-62.

Cet article a été publié dans le « Batna Journal of Medical Sciences » BJMS, l'organe officiel de "I'association de la Recherche Pharmaceutique - Batna »

Le contenu de la Revue est ouvert "Open Access » et permet au lecteur de télécharger, d'utiliser le contenu dans un but personnel ou d'enseignement, sans demander l'autorisation de l'éditeur/auteur.

Avantages à publier dans BJMS :

- Open access : une fois publié, votre article est disponible gratuitement au téléchargement

- Soumission gratuite : pas de frais de soumission, contrairement à la plupart des revues « Open Access "

- Possibilité de publier dans 3 langues : français, anglais, arabe

- Qualité de la relecture : des relecteurs/reviewers indépendants géographiquement, respectant l'anonymat, pour garantir la neutralité et la qualité des manuscrits.

Pour plus d'informations, contacter BatnaJMS@gmail.com

ou connectez-vous sur le site de la revue : www.batnajms.com 


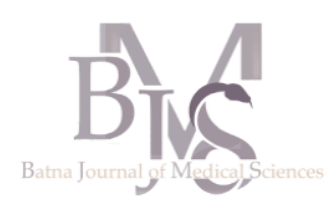

Faculté de Médecine, Université Alger 1, Alger - Algérie

\section{Correspondance à :}

Fadia RAHAL

ffadia 2007@yahoo.fr

DOI :https://doi.org/10.48087/ BJMStf.2015.2115

Il s'agit d'un article en libre accès distribué selon les termes de la licence Creative Commons Attribution International License (CC BY 4.0), qui autorise une utilisation, une distribution et une reproduction sans restriction sur tout support ou format, à condition que l'auteur original et la revue soient dûment crédités.

\section{Maladie de Gaucher}

\section{Gaucher's disease}

\section{Fadia Rahal, Nadjia Brahimi, Aicha Ladjouze-Rezig}

\section{RÉSUMÉ}

La maladie de Gaucher (MG) est une maladie lysosomale due au déficit génétique en bglucosidase, de transmission autosomique récessive; on en distingue trois types. La MG de type 1 , la plus fréquente, se caractérise par une splénomégalie dans $90 \%$ des cas, une cytopénie (essentiellement, anémie et thrombopénie), et une atteinte ostéoarticulaire infiltrative et destructrice qui impacte souvent la qualité de vie des patients. Les deux autres phénotypes incluent une atteinte neurologique, sévère dans le type 2 qui affecte le nourrisson et évolue rapidement vers le décès, et plus lentement progressive dans le type 3 qui comporte également les symptômes du type 1 . Le diagnostic repose sur le dosage de la bglucosidase dans les leucocytes. Cependant, la maladie de Gaucher reste peu connue, et son diagnostic est souvent fait tardivement particulièrement en milieu rhumatologique ou se sont essentiellement les différentes complications ostéarticulaires associées aux manifestations hématologiques qui permettent d'orienter vers cette maladie. Par ailleurs, l'approche thérapeutique dominante est l'enzymothérapie substitutive (ETS), disponible actuellement pour certaines maladies lysosomales.

Mots clés : maladie lysosomale, hépatosplénomegalie, ostéonécrose aseptique, enzymothérapie substitutive

\begin{abstract}
Gaucher's disease (GD) is a lysosomal disease which is due to a genetic defect in bglucosidase, with autosomal recessive transmission; there are 3 types. Type $1 \mathrm{GD}$ is the most frequent and is characterized by splenomegaly in $90 \%$ of cases, cytopenia (mainly anemia and throbopenia) and a musculoskeletal involvement, with infiltration and destruction that can compromise the patient's life. The two other phenotypes include a severe neurological involvement in type 2 that affects infants and is deadly; in type 3 the neurological involvement is less severe and is also associated with features of types 1 . Diagnosis is made based on the dosing of the bglucosidase in leucocytes. However, GD is still not very well known and its diagnosis is made very late, in rheumatological setting in the context of musculoskeletal complications associated with hematological involvement, which allow to guide the diagnosis. Furthermore, the main therapeutic approach includes enzyme replacement therapies, available in some lysosomal diseases.
\end{abstract}

Keywords: Lysosomal disease, hepatoplenomegaly, aseptic necrosis, enzyme replacement therapy.

\section{Pour citer l'article :}

Rahal F, N Brahimi, A Ladjouze-Rezig. Maladie de Gaucher. Batna J Med Sci 2015;2(1):66-69. https://doi.org/10.48087/ BJMStf.2015.2115

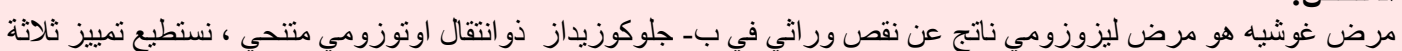

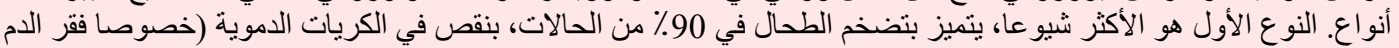

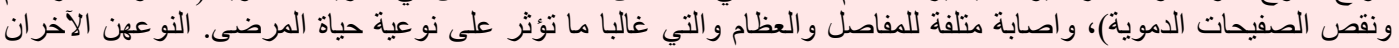

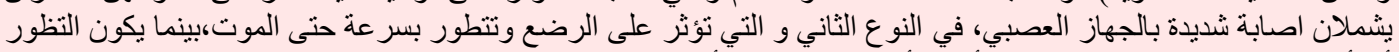

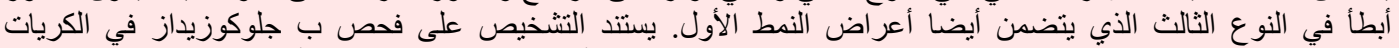

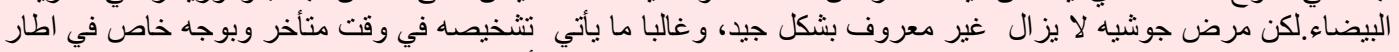

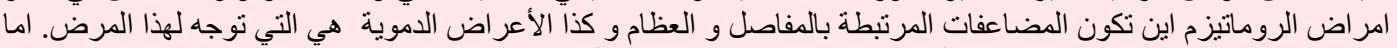

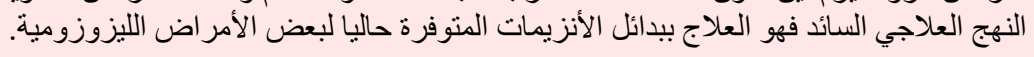
كلمات البحث: المرض الليزوزومي، ضخامة الكبد والطحال، تنخر العظم، العلاج ببدائل الأنزيمات 


\section{INTRODUCTION}

La maladie de Gaucher (MG) est une affection génétique rare, autosomique récessive, due à un déficit de l'activité de l'enzyme lysosomale : la glucocérébrosidase.

Considérée comme la plus fréquente des maladies lysosomales, la MG est héréditaire à transmission autosomique récessive et sa distribution est ubiquitaire [1], elle se caractérise par une hépatosplénomégalie fréquente, une atteinte osseuse parfois sévère et, dans certaines formes rares, une atteinte neurologique [2]. La prévalence de la MG est de l'ordre de 1/60 000 dans la population générale, mais peut atteindre 1/1000 dans la population juive ashkénaze.

\section{PHYSIOPATHOLOGIE}

La glucocérébrosidase est une enzyme lysosomale qui hydrolyse le glucosylcéramide (lipide complexe) en céramide et glucose (figure 1). Dans la maladie de Gaucher, le glucosylcéramide non dégradé s'accumule dans les lysosomes des cellules du système réticulo-endothélial (macrophages), leur donnant un aspect caractéristique en microscopie " cytoplasme en aspect de papier froissé et noyau excentré » (figure 2). Ces macrophages, appelés cellules de Gaucher, vont infiltrer le foie, la rate et la moelle osseuse, et sont responsables des principaux signes cliniques de la maladie [3].

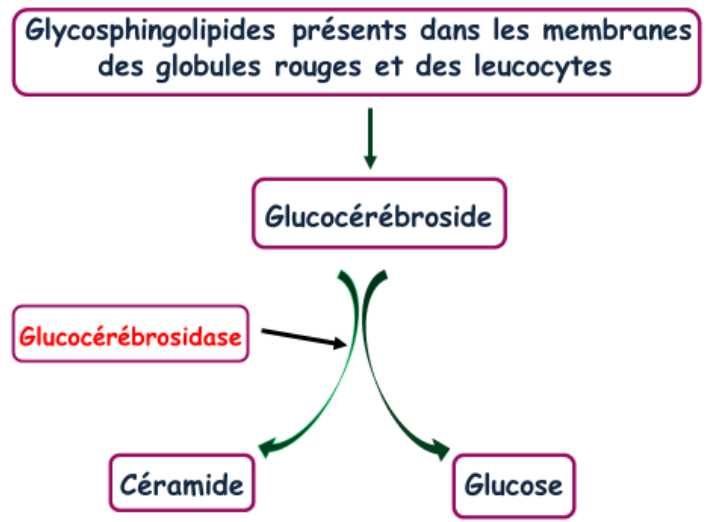

Figure 1 : catabolisme du glucocérébroside

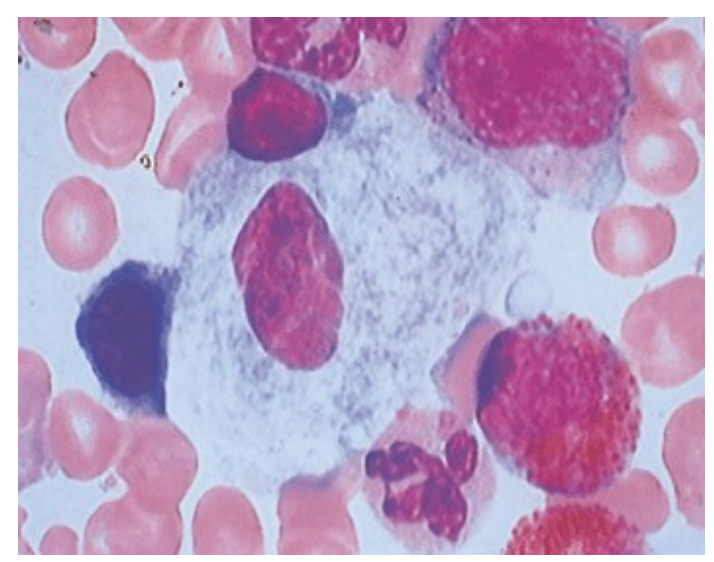

Figure 2 : cellule de Gaucher

\section{DESCRIPTION CLINIQUE}

On décrit classiquement trois phénotypes définis sur des caractéristiques cliniques.

\section{Type 1, sans atteinte neurologique}

Le type 1, défini classiquement par l'absence d'atteinte neurologique [4], est la forme la plus fréquente (95\%). La présentation clinique est très hétérogène, avec parfois des formes asymptomatiques. L'asthénie est fréquente retentissant souvent sur la vie scolaire et socioprofessionnelle. La splénomégalie, parfois majeure, peut aboutir à un véritable infarctus splénique; elle est présente chez $90 \%$ des patients. L'hépatomégalie est notée chez plus de $80 \%$ des patients.

L'hépatosplénomégalie entraîne parfois une distension abdominale douloureuse qui peut comprimer les autres organes et gêner la respiration. L'échographie, et si possible, l'imagerie par résonance magnétique (IRM) abdominale, est un élément important de l'évaluation initiale et de la surveillance.

L'atteinte osseuse est présente dans $80 \%$ des cas et peut retentir sur le pronostic fonctionnel : troubles du remodelage osseux, ostéopénie, ostéoporose, fractures pathologiques et tassements vertébraux (figure 3), lyses osseuses, infarctus osseux se manifestant par des crises douloureuses intenses et invalidantes; ostéonécroses aseptiques (têtes fémorales, têtes humérales, etc.) pouvant évoluer vers une arthropathie dégénérative (figure 4) et justifiant la mise en place d'une prothèse.
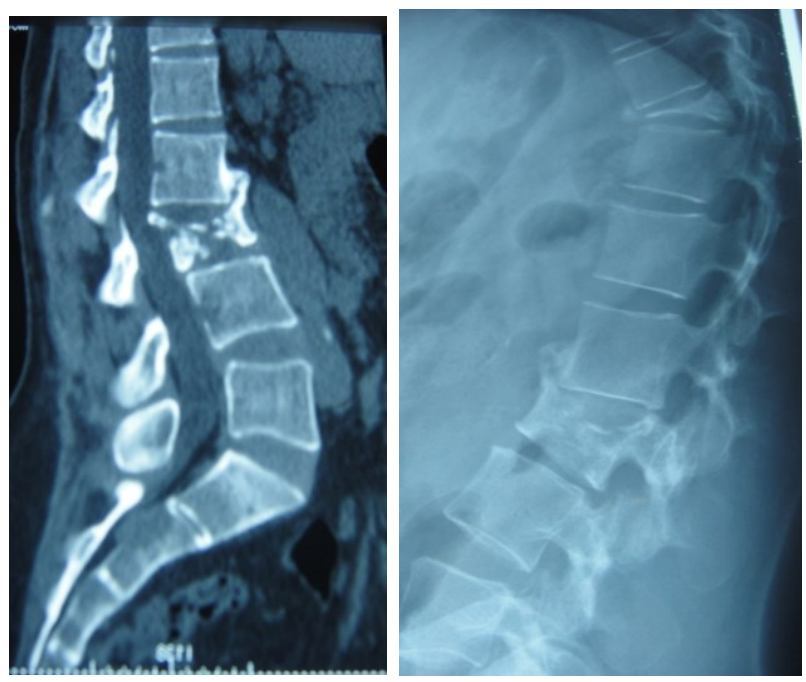

Figure 3 : fractures vertebrales : D11 et L3

L'atteinte des autres organes est rare : atteinte pulmonaire (atteinte interstitielle, fibrose pulmonaire, syndrome restrictif secondaire aux déformations du rachis, hypertension artérielle pulmonaire); atteinte cardiaque (infiltration myocardique ou péricardique, calcifications valvulaires rapportées essentiellement dans le type 3); atteinte rénale se manifestant par une protéinurie et une hématurie, traduisant les dépôts de cellules de Gaucher dans les glomérules ; exceptionnelles atteintes cutanée, oculaire ou digestive.

\section{Type 2, aigu neuronopathique}

Le type $2(1 \%)$, caractérisé par une atteinte neurologique précoce et sévère [5], débute généralement chez le 
nourrisson de trois à six mois par une atteinte systémique avec hépatosplénomégalie et manifestations neurologiques gravissimes.

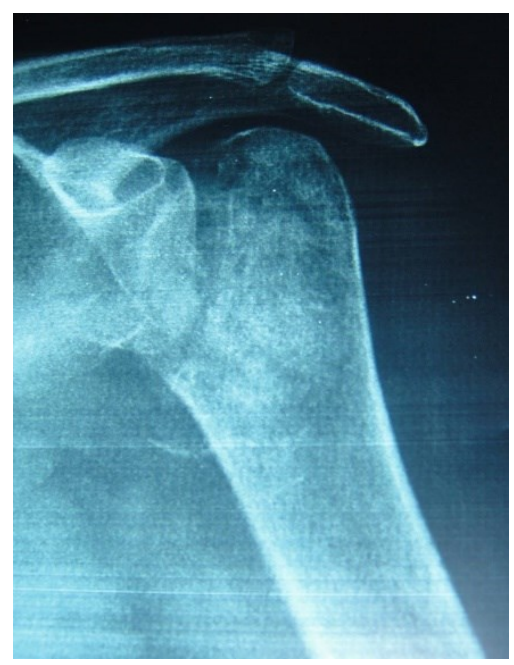

Figure 4 : ostéonécrose de la tête humérale

Les premiers signes sont une paralysie oculomotrice ou un strabisme fixé bilatéral associés secondairement à des signes bulbaires, en particulier des troubles sévères de la déglutition, une spasticité progressive et des mouvements dystoniques. Les convulsions sont plus tardives, se manifestant par une épilepsie myoclonique résistante aux traitements antiépileptiques. Le décès survient le plus souvent avant l'âge de deux ans. Le traitement enzymatique est inefficace.

\section{Type 3}

Le type $3(5 \%)$ est caractérisé par une atteinte neurologique d'apparition plus tardive et d'évolution plus progressive que dans le type 2 [6]. Il est également appelé type juvénile ou type neurologique subaigu. Comme pour le type 1 , il regroupe des malades très hétérogènes. Certains patients ont une atteinte systémique modérée et une ophtalmoplégie associée, comme seul symptôme neurologique. Pour les formes plus sévères les signes neurologiques rencontrés sont variables : ophtalmoplégie horizontale supra nucléaire, épilepsie myoclonique progressive, ataxie cérébelleuse, spasticité et démence dans certains cas. Le traitement enzymatique semble ralentir l'évolution de l'atteinte neurologique. Sans traitement spécifique, l'évolution se fait vers le décès en quelques années.

\section{EXAMENS COMPLÉMENTAIRES POUR DIAGNOSTIC DE LA MALADIE DE GAUCHER}

\section{Myélogramme ou biopsie ostéomédullaire}

Ils permettent de mettre en évidence des cellules de Gaucher, qui sont de grosses cellules macrophagiques dont le cytoplasme est plissé et qui contiennent des inclusions lysosomales constituées de glucosylcéramide. Les cellules de Gaucher ne sont pas spécifiques de la maladie. En effet, on peut retrouver des cellules avec les mêmes caractéristiques cytologiques ("pseudo-Gaucher») dans la leucémie myéloïde chronique, au cours du myélome ou d'autres hémopathies. Le myélogramme permet de caractériser le type de cytopénie.

\section{Dosage de la bêta-glucocérébrosidase}

C'est le dosage de l'activité enzymatique déficiente, il permet de faire le diagnostic. Les valeurs de l'activité enzymatique sont de 10 à $30 \%$ des valeurs normales, parfois moins. Le taux n'est pas prédictif de la sévérité de la maladie.

\section{Recherche de mutations}

On peut rechercher les cinq mutations les plus fréquentes par Polymerase Chain Reaction (PCR). Elles sont parfois un lien avec l'expression clinique de la maladie.

\section{Marqueurs biologiques}

La pancytopénie (par hypersplénisme et/ou infiltration médullaire) associe à des degrés divers une thrombopénie habituelle et rarement profonde, une anémie et une leuconeutropénie (plus rare et peu sévère), Le myélogramme, outre les cellules de Gaucher qu'il peut mettre en évidence, permet d'en préciser le mécanisme soit central par infiltration médullaire, soit périphérique par destruction ou séquestration splénique [7]. L'anémie est souvent au second plan. Les neutropénies sont parfois sévères.

Il existe parfois des troubles de la coagulation à type d'allongement du TCA, sans déficit en facteur de coagulation, dû à l'interférence des sphingolipides avec les protéines de la coagulation ou à une diminution de facteurs de coagulation par consommation par les macrophages activés. Les accidents hémorragiques sont rares en dehors des thrombopénies sévères.

Signes de l'activation macrophagique: Il existe une augmentation de protéines lysosomales comme LAMP-1 (lysosomalassociated protein) et SAP-C (saposine C) dans les formes sévères de la MG.

Actuellement, les deux marqueurs les plus intéressants pour le suivi de la maladie sont la chitotriosidase est le marqueur le plus intéressant, particulièrement pour le suivi du traitement enzymatique. Sa corrélation avec la sévérité de la maladie est bonne quoique imparfaite; un autre marqueur peut être indiqué pour le suivi de la maladie : La chémokine CCL18 est un marqueur intéressant car il est très spécifique de la MG. Son taux peut atteindre jusqu'à 30 fois la normale. C'est un bon marqueur de suivi thérapeutique qui pourrait être dosé chez les patients sans activité chitotriosidase. Cependant ce dosage n'est pas disponible en pratique courante.

\section{Imagerie de l'appareil locomoteur}

\section{Radiographie conventionnelle}

Elle permet d'évoquer le diagnostic, mais les images radiographiques peuvent prêter à confusion avec les hémoglobinopathies et notamment la drépanocytose ou des autres maladies de surcharges (ostéonécrose, ischémie médullaire...).

Scintigraphie osseuse au 99mTechnétium

Non spécifique, elle sert à établir une cartographie des différentes localisations osseuses de la maladie.

\section{Tomodensitométrie}

Elle peut avoir un intérêt pour préciser une atteinte articulaire ou un envahissement des parties molles en cas d'infection.

\section{Imagerie par résonance magnétique (IRM)}

C'est le moyen d'investigation de choix, elle visualise précocement les complications de la maladie : infarctus osseux, ostéonécrose, lésions infectieuses, lésions des parties molles.

\section{PRISE EN CHARGE}

Les objectifs du traitement de la MG de l'adulte sont triples : 
$\checkmark$ Apporter un soulagement fonctionnel;

$\checkmark$ Améliorer la qualité de vie des patients ;

$\checkmark$ Prévenir les complications.

En matière d'atteinte ostéarticulaire, les objectifs thérapeutiques sont précis :

$\checkmark$ Diminuer ou supprimer les douleurs osseuses dans un délai de un à deux ans ;

$\checkmark$ Prévenir les crises douloureuses osseuses;

$\checkmark$ Prévenir les ostéonécroses et les fractures souschondrales ;

$\checkmark$ Augmenter la DMO trabéculaire dans un délai de trois à cinq ans.

\section{Traitement spécifique}

Actuellement, deux approches thérapeutiques spécifiques sont à notre disposition. Le traitement spécifique n'est pas justifié chez tous les patients. Lorsqu'il est commencé, le traitement doit cependant être poursuivi à vie.

Le traitement de première intention de la maladie de Gaucher de type 1 symptomatique et de la maladie de Gaucher de type 3 est l'enzymothérapie de substitution (8), qui permet la dégradation du glycosylcéramide accumulé, améliorant ainsi les anomalies hématologiques, l'hépatosplénomégalie et la qualité de vie en quelques mois. Deux enzymes ont actuellement obtenu l'autorisation de mise sur le marché (AMM): imiglucérase (Cerezyme) et vélaglucérase (VPRIV). Les enzymes sont dispensés en perfusions tous les 15 jours.

Le traitement par réduction de substrat, miglustat (Zavesca) diminue l'accumulation du glucosylcéramide en excès.

L'efficacité de ce traitement administré par voie orale est moins importante que le traitement enzymatique, et les effets secondaires plus fréquents ; c'est donc un traitement de seconde intention.

\section{Surveillance}

Le suivi des patients nécessite, outre le suivi clinique régulier (tous les 3 à 6 mois), un suivi biologique comportant notamment les différents marqueurs (tous les 6 à 12 mois), un suivi radiologique osseux (IRM) tous les 6 mois au début, puis tous les 24 mois après stabilisation, et abdominal tous les 12 à 24 mois (échographie, IRM), une échographie cardiaque initiale et tous les ans en cas d'atteinte cardiaque.

\section{PRONOSTIC}

Le pronostic vital de la maladie de Gaucher de type 1 n'est en général plus engagé depuis l'utilisation du traitement enzymatique substitutif ; cependant, le pronostic fonctionnel est parfois médiocre compte tenu des possibles séquelles liées à l'atteinte osseuse et des pathologies associées à la maladie (myélome, lymphome, syndrome parkinsonien).

Déclaration d'intérêts : les auteurs ne déclarent aucun conflit d'intérêt en rapport avec cet article.

\section{RÉFÉRENCES}

1. Grabowski GA. Phenotype, diagnosis, and treatment of Gaucher's disease. Lancet 2008;372:1263-71.

2. Grabowski GA. Recent clinical progress in Gaucher disease. Curr Opin Pediatr 2005;17:519-24.

3. Charrow J, Andersson HC, Kaplan P, et al. The Gaucher registry: demographics and disease characteristics of 1698 patients with Gaucher disease. Arch Intern Med 2000; 160: 2835-43

4. Erikson A, Bembi B, Schiffmann R. Neuronopathic forms of Gaucher's disease. Baillieres Clin Haematol 1997 ; 10(4):711-23.

5. Mignot C, Gelot A, Bessieres B, Daffos F, Voyer M, Menez F, et al. Perinatallethal Gaucher disease. Am J Med Genet A 2003 ; 120 (3) : 338-44.

6. Mignot C. Aspects cliniques des formes neurologiques précoces de maladie de Gaucher. Rev Med Interne 2006 ; (Suppl 1):S14-7.

7. Stirnemann J, Caubel I, Kettaneh A, Fain O, Belmatoug N. Aspects épidémiologiques, cliniques, biologiques et thérapeutiques dans la maladie de Gaucher. Presse Med 2003;32 (11):503-11.

8. Barton NW, Brady RO, Dambrosia JM, et al. Replacement therapy for inherited enzyme deficiency-macrophage-targeted glucocerebrosidase for Gaucher's disease. N Engl J Med. 1991;324:1464-70.

Cet article a été publié dans le « Batna Journal of Medical Sciences » BJMS, l'organe officiel de "I'association de la Recherche Pharmaceutique - Batna "

Le contenu de la Revue est ouvert « Open Access » et permet au lecteur de télécharger, d'utiliser le contenu dans un but personnel ou d'enseignement, sans demander l'autorisation de l'éditeur/auteur.

Avantages à publier dans BJMS :

- Open access : une fois publié, votre article est disponible gratuitement au téléchargement

- Soumission gratuite : pas de frais de soumission, contrairement à la plupart des revues « Open Access"

- Possibilité de publier dans 3 langues : français, anglais, arabe

- Qualité de la relecture : des relecteurs/reviewers indépendants géographiquement, respectant l'anonymat, pour garantir la neutralité et la qualité des manuscrits.

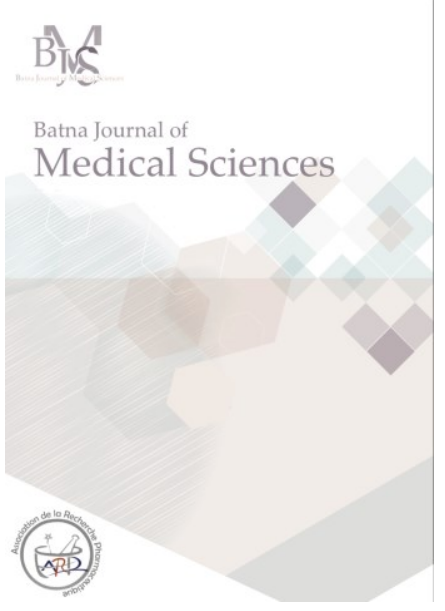




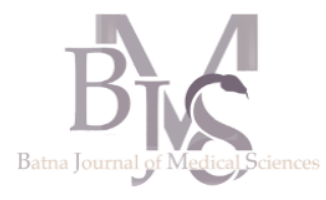

\title{
La maladie génétique et la famille : fatalité ou culpabilité ?
}

\author{
The hereditary disease and family : fatality or culpability?
}

\author{
Hanifa Salhi
}

Maitre de Conférence en psychologie clinique Département de Psychologie, Université Hadj Lakhdar Batna, Algérie

\section{Correspondance à :}

Hanifa SALHI

salhi.hanifa@yahoo.fr

DOI :https://doi.org/10.48087/ B]MStf.2015.2116

Il s'agit d'un article en libre accès distribué selon les termes de la licence Creative Commons Attribution International License (CC BY 4.0), qui autorise une utilisation, une distribution et une reproduction sans restriction sur tout support ou format, à condition que l'auteur original et la revue soient dûment crédités.

\section{RÉSUMÉ}

L'annonce d'une maladie rare chez un enfant est le début d'un chemin long et tortueux, pour lui et pour ses proches. Généralement, les parents apprennent la maladie de leur enfant alors que ce dernier est encore un nourrisson en bonne santé. Les réactions des familles face à l'annonce du diagnostic sont très variables. Certains parents semblent accepter la maladie souvent comme forme de soumission au «maktoub » (fatalisme), d'autres à l'extrême, se séparent dans la douleur et la culpabilité peu de temps après l'annonce, ou même après des années de souffrance. Mais dans tous les cas, l'histoire de la famille semble s'articuler autour de la révélation de la maladie, pour laquelle, rappelons le, les symptômes sont invisibles, ce qui laisse une place importante aux fantasmes les plus redoutables. Les sentiments d'insécurité, de précarité en face d'un tel dilemme s'installent, et la nécessité d'avoir du temps pour faire le deuil de l'enfant classique, normal font aggraver le vécu de la famille, qui s'apprête -comme un système en évolution- à faire face à un déséquilibre systémique. Souvent ce n'est pas tellement les difficultés ou les handicaps de l'enfant qui sont difficiles pour les parents, mais le fait qu'il soit malade, qu'ils n'arrivent pas à assimiler les effets secondaires des médicaments, qu'ils sont incapables de prévoir son avenir. Donner la vie et la maladie est un paradoxe difficile à vivre dans ce genre de situation. Un suivi médical s'inscrit dans la durée (bilans annuels, soins), et dans de rares services hospitaliers, il est possible pour les familles de rencontrer le psychologue lorsqu'elles le souhaitent. Le soutien psychologique est fort recommandé devant un vécu qui dévie entre un sentiment de culpabilité intense et un sentiment d'impuissance et de soumission devant la maladie qui peut vouer à l'échec tout le processus de prise en charge médicale.

Mots-clés : Maladie rare, hérédité, famille, réaction.

\begin{abstract}
Announcing a rare disease in a child is the bginning of a long and tortuous way for the patient as well as his parents. Globally, parents learn about the disease of their child since he is still very young and in good health. Reactions of the family when the diagnosis is announced are very variable. Some parents seem to accept disease in a kind of submission or resignation "Maktoob", and on the other side, will separate shortly after or after years of suffering. In all cases, the history of the family seems to be articulated around the revealing of the disease, for which, symptoms are not visible yet, so all kinds of dangerous fantasies. Feelings of insecurity in front of such a dilemma come and the necessity to have enough time to mourn the "classical child" will worsen the experience of the family, which expect to systematically face a systematic imbalance. Often, difficulties do not come from the child's handicap but from the fact that they are unable to assimilate the side effects of the different drugs his is taking, which are not capable of predicting his future. Giving life as well as illness is a dilemma that is difficult to live in this kind of situation. A long-time medial follow-up with annual check-ups and various medical care, provided only in very few hospital departments, it is possible for family members to get in touch with psychologists, if they wish. Psychological support is highly recommended in front of a situation of intensive culpability and of incapacity or submission, which can lead to a failure in the process of medical management of the patient.
\end{abstract}

Keywords: Rare disease, heredity, family, reaction.

\section{Pour citer l'article :}

Salhi H. La maladie génétique et la famille : fatalité ou culpabilité ? Batna J Med Sci 2015;2(1):70-74. https://doi.org/10.48087/ BJMStf.2015.2116

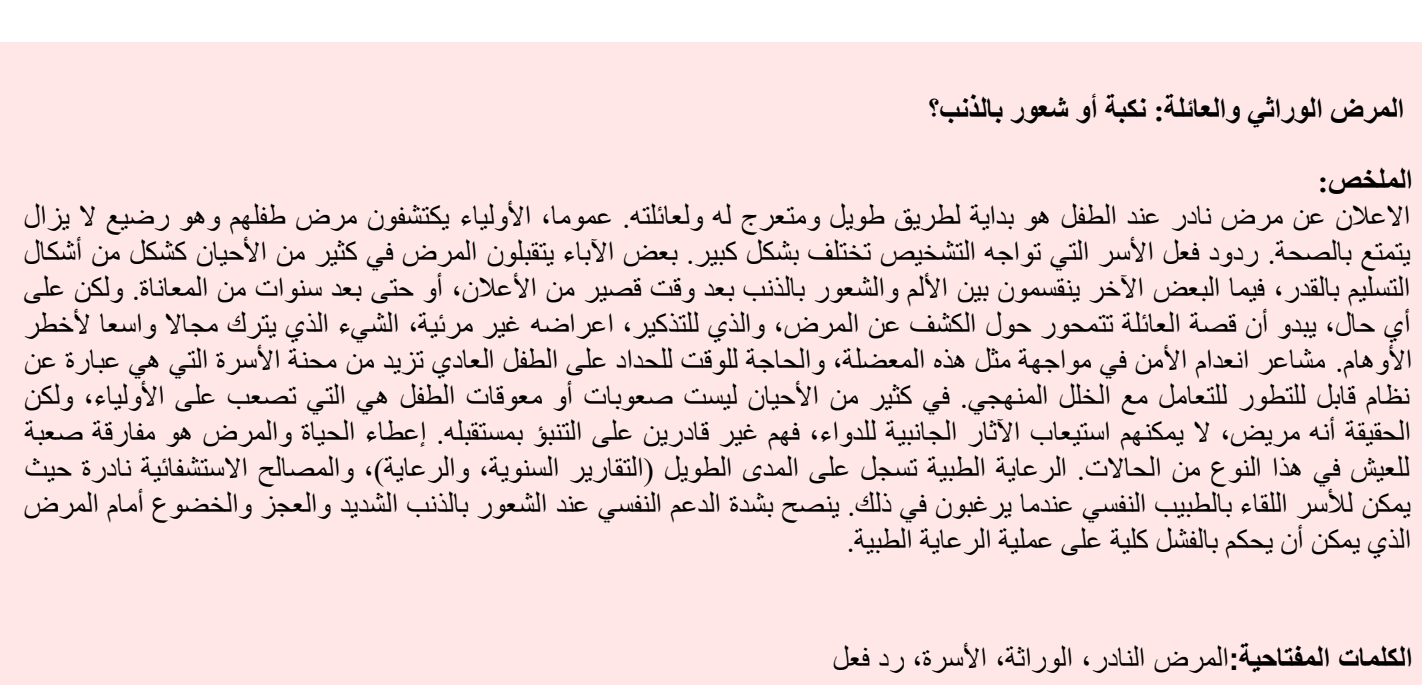




\section{INTRODUCTION}

Une maladie se définit par une altération de l'état de santé, elle désigne un état morbide dont on connaît le plus souvent la cause, mais quelques soient sa durée et sa gravité, elle marque la vie psychologique et sociale de l'individu. C'est une épreuve, une crise, qui modifie une situation relationnelle préalablement établie. Les réactions à la maladie ne peuvent être considérées comme des épiphénomènes, elles font partie intégrante du processus de maladie lui-même, influençant parfois, de façon inquiétante, son évolution et son pronostic.

Entendre un diagnostic de maladie génétique qu'il soit pour un adulte ou pour un enfant est vécu par le malade et ses proches comme quelque chose de brutal et de destructeur. Même si les conditions de cette annonce furent l'une des meilleures (choix du moment, des mots, compassion du médecin) cela signe une rupture, une catastrophe avec, parfois, des sensations physiques de bascule, de désorientation temporo-spatiale.

Le vécu d'un tel moment peut -être attribué à une blessure narcissique intense, un effondrement qui s'accompagne d'un fort sentiment de culpabilité, avec parfois des constructions imaginaires non rationnelles. L'annonce du diagnostic reste presque toujours gravée dans les mémoires comme une mauvaise nouvelle marquant la fin d'un temps de vie où la maladie était absente, voire inimaginable. Car, même si l'annonce a été précédée d'une période d'investigations et d'examens, cela ne peut toujours amortir le choc de la "révélation», il peut aussi être source d'un grand soulagement: il signe le point de départ d'un nouvel engagement, d'une reconstruction... dans les meilleurs des cas.

Le diagnostic de maladie génétique est un moment clé de l'histoire de la famille qui se trouve «atteinte» dans sa totalité : les malades, qui vont devoir "gérer» au quotidien une maladie douloureuse, invisible pour l'entourage; les parents qui, après l'annonce du diagnostic, vont devoir réaménager leur vie et composer avec les changements que la maladie engendre au sein du foyer familial; les frères et sœurs qui vont devoir se repositionner par rapport à ce frère ou cette sœur malade et essayer de trouver des réponses à la multitude de questions qu'ils se posent, et enfin, les grandsparents qui peuvent éprouver une certaine culpabilité et se demandent souvent comment aider les parents.

Tout le rôle de l'approche psychologique dans ces maladies est alors de donner ou de redonner une place à chaque membre de la famille.

\section{LA MALADIE GENETIQUE OU LA SOUFFRANCE CHRONIQUE}

Comme il est défini théoriquement : «les maladies génétiques désignent l'ensemble des maladies qui sont causées par un ou plusieurs gènes défectueux ou par une anomalie chromosomique, elles peuvent être héréditaires ou non. Ainsi, les maladies génétiques qui concernent des gènes défectueux sont transmissibles à la descendance. Les maladies chromosomiques, elles, ne sont pas héréditaires (la trisomie 21 constitue le cas le plus répandu d'anomalie du nombre de chromosomes, les parents d'un enfant atteint ne sont pas porteurs de l'anomalie génétique). » [1]

En dépit de leur grande hétérogénéité, les maladies génétiques - à l'instar des maladies chroniques - présentent des caractéristiques assez communes, ce sont des maladies :
- invalidantes, avec une qualité de vie dégradée et une perte d'autonomie ;

- la douleur du malade occupe une place importante ;

- pour lesquelles il n'existe pas de traitement, mais des moyens pour soulager les symptômes, ce qui améliore quelque peu la qualité de vie ;

- parfois rares, évolutives, chroniques

- avec mise en jeu du pronostic vital.

Les réactions face à la maladie sont diverses et dépendent de nombreux facteurs qui concernent le malade lui-même (âge, sexe, position dans la famille, fonctionnement psychique antérieur, modification de l'image corporelle) ou des facteurs qui se rattachent à son entourage proche (personnalité parentale, modes relationnels parents-enfant, histoire familiale quant à la maladie...etc.)

Le souci majeur tourne autour du devenir de ces malades, surtout ceux atteints à un bas âge qui se trouvent condamnés toute une vie par l'aspect chronique et invalidant de ces maladies. Une étude effectuée par la Fondation WYETH en France [2] a pris pour point de départ le souci du vécu psycho-social des malades jeunes : «Si la médecine clinique, avec de nouvelles molécules, une meilleure prise en charge thérapeutique et chirurgicale sait mieux traiter les conséquences physiologiques et fonctionnelles de ces maladies, le traitement de l'impact psychologique, social et sociétal d'une vie " sous contrôle médical » est encore trop souvent négligé.» [3]. L'étude comprend 20 interviews de jeunes âgés de 12 à 25 ans et 10 interviews de parents d'enfants de moins de 18 ans. Les entretiens réalisés visaient l'illustration de diverses situations :

- selon la visibilité pour autrui,

- selon la notoriété, la connaissance de la maladie par autrui,

- selon le poids et la nature des traitements et des soins,

- selon l’origine de la maladie (acquise, génétique),

- selon le pronostic vital de la maladie chronique.

Les opinions dégagées surtout des enfants et adolescents peuvent être résumées comme suit :

$\checkmark$ « C'est une maladie qui se répète, qui normalement, dans la plupart des cas, je pense, ne peut pas se soigner.»

$\checkmark$ «Ils ont commencé à dire que c'était ma mère et mon père qui m'empoisonnaient»

$\checkmark$ «On était bien contents de savoir, qu'on puisse enfin dire « elle a ça donc on va pouvoir faire ça, on va lui donner ça comme médicaments, elle est dans tel stade.» (avis d'un parent)

$\checkmark$ «Si les parents, ils ne savent pas tout ce qu'il faut, ils ne peuvent pas aider l'enfant» (avis d'un soignant)

$\checkmark$ « C'est important pour moi d'être autonome déjà pour pouvoir faire ma vie.»

Une tendance nette vers cette volonté d'autonomie et de cohabitation avec la maladie, renforcée par l'évolution galopante de la médecine qui offre désormais aux parents et aux proches des alternatives plus éclairées de la façon dont ils peuvent aider le malade. Les tests de dépistage de certaines maladies génétiques sont rendus possibles par les progrès de la biologie moléculaire. Dans certains cas, il est possible de traiter des carences d'ordre génétique par un régime approprié.

\section{LES SEQUELES PSYCHOLOGIQUES ET SOCIALES :}

La maladie génétique chronique est douloureuse, éprouvante, souvent épuisante, d'évolution variable et difficilement 
prévisible, potentiellement invalidante. Douleurs aiguës et chroniques, souffrance morale : il faut insister sur la sévérité et la spécificité des douleurs et des handicaps, dont les conséquences psychologiques, familiales, sociales, peuvent être encore mal analysées voire incomprises.

Le vécu de chaque malade est donc particulièrement important à connaître et reconnaître. La prise en charge est d'autant plus efficace qu'elle est centrée sur le patient, son vécu, ses besoins, ses croyances, chaque personne étant prise dans sa globalité, d'où l'approche systémique s'impose comme démarche de prise en charge multidimensionnelle.

L'annonce du diagnostic (le "verdict") constitue un choc, voire un traumatisme difficile à surmonter, mais permet aussi de mettre un nom sur la maladie, l'identifier et l'appréhender parfois après une période pénible d'incertitude. Une succession d'étapes psychologiques est alors franchie par chaque personne, à un rythme qui lui est propre : choc, dénégation, révolte, puis prise de conscience progressive, réflexion ; finalement une dynamique constructive s'installe : le patient doit apprendre à gérer activement la maladie par des stratégies (coping) qui lui sont propres (selon son historique, sa personnalité, ses compétences...) et son nouvel équilibre de vie, aux niveaux personnel, familial, social et professionnel.

Le patient doit sans cesse "inventer son propre chemin". Les stratégies actives de "coping", centrées sur la résolution des problèmes (recherche d'information, contrôle de la douleur, organisation du planning quotidien et du "réseau de soins"...) sont associées à un vécu positif, souvent une conséquence du soutien familial lui même dépendant du mode de relation établi entre le malade, la famille et l'équipe soignante. Par contre les stratégies basées sur la recherche de compassion d'autrui freinent les capacités d'adaptation et d'affrontement chez le malade en état de dépendance émotionnelle et affective vis-à-vis l'entourage. Beaucoup de patients reconnaissent d'autre part des aspects positifs à leur maladie et lui trouvent un sens : meilleure écoute des autres, plus grande sérénité vis à vis des petits maux de la vie, plus grande force morale... là où la fatalité peut jouer un rôle de versets différents!

Cette lutte constante pour surmonter des difficultés parfois majeures dans l'accomplissement de chacun des gestes de la vie quotidienne, l'incapacité à faire des projets, sont vécues souvent dans un sentiment de solitude et d'incompréhension, de dévalorisation, même si l'entourage, qui joue un rôle capital, est présent. On comprend bien la fréquence de l'anxiété (craintes pour l'avenir, douleurs, handicap, isolement, pertes affectives, sociales, professionnelles...) et l'apparition possible d'une dépression, un retrait avec repli sur soi, qui doit être reconnu et traité, qu'il soit chez le malade ou chez l'un de ses proches.

\section{Les réactions parentales :}

«L'annonce de la maladie sépare un avant et un après : un avant mythique où rien de la souffrance n'existait et un après angoissant, inquiétant. Les réactions des parents dépendent de leur propre histoire - individuelle, de couple, de famille Que s'est-il passé avant l'arrivée de cet enfant ?... » [4]

Angoisse: peut devenir majeure surtout s'il s'agit des maladies qui engagent le pronostic vital, ou quand le risque de l'handicap est majeur (ex : l'épilepsie). A côté de l'angoisse de mort s'ajoute un ressenti d'impuissance, la situation est vécue «comme une sanction quelque peu démoniaque, inquiétante, proche de la folie » [5]. Les réactions face à cette angoisse peuvent conduire à la limitation non justifiée des activités de l'enfant, une forme de surprotection qui révèle l'ambivalence parentale, ou au contraire on peut assister à une permissivité excessive par peur que chaque contrariété est sensée aggraver l'état de l'enfant... un sentiment de toute puissance qui peut nuire à l'équilibre psychologique de l'enfant (problème de limites) et entraver son cursus scolaire. Parfois l'angoisse provoquée par la maladie vient révéler des crises préexistantes masquées par un apparent équilibre familial qui s'écroule subitement (séparation parentale, décompensation de l'un des parents ou l'un des membres de la famille souvent sur un mode dépressif...etc.)

Dépression: elle est souvent maternelle, elle peut être purement réactionnelle, comme elle peut s'inscrire dans un processus plus complexe où la maladie n'est qu'un catalyseur. Les réactions peuvent se manifester par une fuite de l'affrontement avec l'enfant malade par peur de s'écrouler psychiquement, ce qui donne parfois l'image de «mauvais parent » auprès de l'équipe soignante et peut même enraciner l'idée fausse de l'incapacité d'aider l'enfant malade. Le cas contraire est largement reconnaissable par les médecins qui affrontent souvent des parents exigeants et intransigeants, tellement présents avec leur enfant, ce qui empêche ce dernier de nouer des relations propres avec les personnes qui le soignent. L'exigence des parents peut aller à l'encontre de l'enfant en lui demandant d'assumer sa maladie et le mal qu'elle engendre chez eux, ne lui permettant pas d'exprimer ses souffrances et sa douleur, ce qui est néfaste pour lui. Il se voit contraint d'adopter un faux self hyper-mâture prédisposé à la décompensation psychique de tout genre et à tout moment.

Agressivité : liée souvent au sentiment de culpabilité dû à une ambivalence entre la blessure narcissique et l'effondrement du fantasme de l'enfant imaginaire ce qui les conduit à considérer tout signe de la maladie comme une agression dirigée contre eux directement. Le sentiment d'échec face aux parents des parents (grands-parents) qui n'ont pas su comme leurs prédécesseurs protéger leur progéniture. La perception d'une punition - méritée ou pas inconsciemment - fait augmenter les tendances agressives souvent masquées ou déniées sous forme de surprotection (renforcement des contraintes et des interdits, rigorisme thérapeutique obsessionnel que l'enfant peut refuser par des conduites d'opposition), ou un rejet direct de l'enfant ou de l'équipe soignante dont les compétences sont remises en cause.

Culpabilité : le caractère génétique de la maladie donne l'impression d'avoir transmis une tare à son enfant et certifie une part de responsabilité dans le processus de la maladie. Les manifestations sont identiques à celles de l'agressivité, tellement entremêlées comme sentiments.

\section{Les réactions de la fratrie :}

Le sentiment de rivalité, normal dans les relations de fratrie se trouve bouleversé dans le cas d'un frère ou sœur malade. Même devant une jalousie légitime face à un intérêt majeur accordé à l'enfant malade, les frères et sœurs sont appelés au contraire à manifester un sentiment maternel envers cet enfant fragile et faible. Une culpabilité envahissante se fait sentir devant une agressivité "légitime » ce qui ramène le vécu affectif et émotionnel de toute la famille à un déséquilibre pathogène, si la prise en charge psychologique ne fait pas irruption à tout ce système, donc «le principal danger pour la fratrie est de ne pas pouvoir préserver sa place, ses désirs propres, ses réactions agressives normales et leur élaboration » [6]. 


\section{LA PRISE EN CHARGE MULTIDISCIPLINAIRE :}

La génétique médicale est un sujet complexe, qui nécessite un accompagnement particulier. Lors du parcours de soin, le malade et sa famille rencontrent plusieurs professionnels spécialisés dans ce domaine. En qualité d'intervenant on peut citer :

\section{Le généticien clinicien : la consultation \\ Le médecin généticien assure plusieurs rôles :}

Rôle de diagnostic : le médecin généticien reçoit les patients en consultation, dès le début du parcours de soin, d'où l'importance de son intervention dans tout le processus thérapeutique. Il établit l'arbre généalogique du patient et recense ses antécédents familiaux. Il examine le patient et peut prescrire des examens complémentaires afin de compléter son diagnostic (radiographies, échographies, etc.). Il informe le patient, ou ses parents s'il est mineur, des modalités du test génétique et de ses conséquences psychologiques et thérapeutiques. Selon la maladie suspectée et les souhaits du patient, il prescrit ensuite l'analyse génétique adaptée. Au sein de l'hôpital, le généticien peut donner des consultations dans tous les services et voir des patients aux pathologies très variées: retard mental, maladies neurologiques, pathologies immunitaires, malformations, troubles sensoriels, certains cancers, etc.

Rôle de prévention : le généticien peut dépister des sujets à risque de transmission à leur descendance d'une maladie génétique grave et incurable identifiée dans leur famille (par exemple mucoviscidose, myopathie). S'ils sont porteurs de cette maladie et s'ils le souhaitent, le médecin organise et coordonne le diagnostic prénatal. Il peut dépister les sujets à risque de développer eux-mêmes une maladie liée à une anomalie génétique identifiée dans leur famille (par exemple prédisposition génétique au cancer). L'objectif est de mettre en place chez ceux qui se révèlent porteurs de l'anomalie génétique les mesures de prévention et de soin. On parle alors de «médecine prédictive ».

Rôle de coordination : après avoir établi son diagnostic, le médecin généticien oriente son patient, dont la prise en charge peut nécessiter de consulter de nombreux autres spécialistes. Il apporte également une aide dans le soutien psychologique et social des patients, en les mettant en relation avec des professionnels spécialisés et en les aidant à trouver des structures et des associations pour une meilleure gestion de la vie quotidienne. Le dialogue médecin / malade est important à toutes les phases de la maladie. Il s'agit d'un véritable "contrat de confiance" basé sur l'information réciproque et le respect mutuel.

\section{Le généticien biologiste : le laboratoire}

Le généticien biologiste réalise les analyses biologiques génétiques. Il peut effectuer des analyses chromosomiques (cytogénéticien) ou géniques (généticien moléculaire). Il vérifie les résultats des examens et met au point les tests diagnostiques pour les anomalies génétiques nouvellement identifiées. En raison de la haute technicité des analyses effectuées et de la particularité des informations obtenues il doit faire preuve de rigueur et d'assurance en même temps.

\section{Le conseiller en génétique}

Le conseiller en génétique occupe, en quelque sorte, une place d'intermédiaire entre le patient et le médecin généticien qui le suit. Son rôle est en effet d'aider le patient à mieux comprendre les implications des résultats d'une analyse génétique pour lui et pour sa famille, et de présenter les risques éventuels de la transmission de la maladie à ses enfants. Après la réalisation du test génétique, il informe également les patients sur leur prise en charge médicale et les thérapies possibles. A l'écoute du malade, il peut apporter un soutien psychologique autant qu'un suivi, dans le respect des conditions éthiques, sociales et culturelles.

\section{Le psychologue}

Le rôle du psychologue est d'accompagner les patients, qui peuvent être confrontés à des situations délicates : maladies évolutives entraînant des dysfonctionnements moteurs et cognitifs, maladies rares difficiles à diagnostiquer, maladies génétiques incurables, etc. Le psychologue est là pour apporter le soutien nécessaire aux patients afin de les préparer et de les accompagner au mieux, à la fois pour gérer la maladie sur le plan individuel mais aussi pour en gérer les répercussions familiales. La consultation avec le psychologue est proposée au patient et à sa famille.

Le besoin d'information, de reconnaissance, ne pas être seul et bénéficier de la prise en charge optimale, incluant les innovations thérapeutiques : c'est tout l'enjeu des relations avec l'équipe soignante et les associations. La qualité du dialogue médecin / malade est essentielle tout au long de l'évolution de la maladie, la relation de confiance qui doit s'instaurer entre les deux - où le patient joue un rôle de plus en plus actif et responsable, tant au niveau personnel qu'associatif - est capitale pour le processus thérapeutique.

Chaque poussée, chaque changement thérapeutique, chaque décision chirurgicale sont autant d'étapes importantes à gérer par la suite par toute l'équipe. La consultation constitue un moment privilégié de dialogue, de prise de décisions importantes... avec aussi un risque de déception, tant les attentes sont fortes.

La qualité de l'information du patient et de son entourage est capitale : une meilleure compréhension de la maladie, de ses retentissements, de ses traitements, permet de dédramatiser et d'aller de l'avant. Cette information est une des attentes majeures du patient, un besoin et un droit. Elle doit être personnalisée, claire et complète, évolutive ; il faut expliquer, rassurer, insister sur les points essentiels, vérifier la compréhension ; au dialogue peuvent s'ajouter des documents écrits. Elle aboutit au consentement éclairé et à la décision partagée, qui a le plus de chance de succès.

L'information se prolonge par une véritable éducation thérapeutique, démarche éducative structurée qui permet au malade d'acquérir savoir, savoir-faire et savoir être, pour mobiliser toutes ses ressources et réussir le "coping". Nul ne peut nier les effets positifs sur l'observance, l'état physique, psychique, le recours aux soins et même le pronostic général.

Le patient devient ainsi un expert et un gestionnaire actif de sa maladie. Il est membre à part entière de l'équipe multidisciplinaire (outre les médecins : infirmiers, kinésithérapeutes, psychologues, assistantes sociales...) impliqué dans la prise en charge globale personnalisée. 


\section{CONCLUSION}

La participation active des patients fait partie des grands axes de la démarche de multi partenariat que doit mettre en œuvre l'Etat pour une prise en charge effective et efficace des malades génétiques. Il faut saisir que tant que le malade se sent une partie prenante dans ce programme ainsi que son entourage, les perspectives d'une meilleure gestion de ces maladies peuvent voir le jour. Dépasser le sentiment de culpabilité et l'impuissance devant une fatalité sévère est une motivation de cohabiter avec la maladie et pourquoi ne pas y faire face. En Occident, des " Programmes Patient Partenaire " sont mis en marche et dans lesquels des patients volontaires et formés pourraient peut-être alors enseigner avec succès le vécu de leur maladie, notamment aux étudiants en médecine.

Les Associations de patients qui peuvent intégrer des parents de personnes - et notamment d'enfants - malades jouent un rôle important. Leur mission première est d'informer leurs adhérents, de les aider à trouver un nouvel équilibre. L'information du public, actuellement nulle, devient particulièrement importante, car elle permettrait de changer l'image de ces maladies qui souffrent encore de nombreuses idées fausses.

Les associations sont aussi des interlocuteurs privilégiés pour les professionnels de santé et pour la société civile, et devraient intervenir au niveau des différents leviers de décisions. L'apparition de thérapeutiques innovantes, d'efficacité prouvée chez les malades les plus sévèrement atteints, mais de coût élevé, source d'interrogations éthiques et médico-économiques, devrait renforcer toute forme de multi partenariat. Il s'agit bien d'une priorité de santé publique... Au total, la prise en compte du vécu et de la dimension psychosociale est indispensable. C'est en alliant la qualité humaine du dialogue aux avancées des traitements et des stratégies que l'on pourra répondre pleinement aux attentes légitimes des patients et de leurs familles.
Déclaration d'intérêts : l'auteur ne déclare aucun conflit d'intérêt en rapport avec cet article.

\section{RÉFÉRENCES}

1. "Maladie génétique - Définition », Sante-Médecine, Réalisé en collaboration avec des professionnels de la santé et de la médecine, sous la direction du docteur Pierrick HORDE, Juin 2014.

2. Maladies chroniques de l'enfant et de l'adolescent « Une vie à construire ", Conférence $\mathrm{N}^{\circ} 1$, DIAGNOSTIC ET PREMIERS TRAITEMENTS,UN PROJET DE VIE FRACTURE ?. Mardi 16 janvier 2007.

3. Maladies chroniques de l'enfant et de l'adolescent " Une vie à construire ", Conférence $N^{\circ} 2$. Mardi 16 janvier 2007.

4. Faure J, Romero M. Retentissements psychologiques de la drépanocytose. Seen on http://lesouriredeselasse.free.fr/telecha rgement /Retentissements-psychologiques-drepanocytose.pdf

5. Epelbaum C, Ferrari P. Réactions psychologiques à la maladie chez I'enfant. In : Ferrari P, Epelbaum C, eds. Psychiatrie de l'enfant et de I'adolescent. Paris : Médecine-Sciences, Flammarion, 1993. p53

6. Epelbaum C, Ferrari P. Réactions psychologiques à la maladie chez I'enfant. In : Ferrari P, Epelbaum C, eds. Psychiatrie de l'enfant et de I'adolescent. Paris : Médecine-Sciences, Flammarion, 1993. p445.

7. Lacombe D., Toussaint E., 2007, « Annonce du diagnostic d'une maladie génétique et prise en charge psychologique des patients et de leur entourage ", La Presse médicale, 36 : 1S20-1S25.

8. Association Française pour la Recherche sur l'hidrosadénite. Accédé sur http://www.afrh.fr/la_maladie_de_verneuil_ou_h/vecu.html, consulté le 12/08/2014

9. Les maladies rares en bref : entre communauté et diversité. Accédé sur http://www.hopital.fr/Hopitaux/Vos-dossiers-sante/Maladiesrares2, consulté le 23/09/2014

Cet article a été publié dans le « Batna Journal of Medical Sciences » BJMS, I'organe officiel de "l'association de la Recherche Pharmaceutique - Batna "

Le contenu de la Revue est ouvert « Open Access » et permet au lecteur de télécharger, d'utiliser le contenu dans un but personnel ou d'enseignement, sans demander l'autorisation de l'éditeur/auteur.

Avantages à publier dans BJMS :

- Open access : une fois publié, votre article est disponible gratuitement au téléchargement

- Soumission gratuite : pas de frais de soumission, contrairement à la plupart des revues "Open

Access »

- Possibilité de publier dans 3 langues : français, anglais, arabe

- Qualité de la relecture : des relecteurs/reviewers indépendants géographiquement, respectant l'anonymat, pour garantir la neutralité et la qualité des manuscrits.

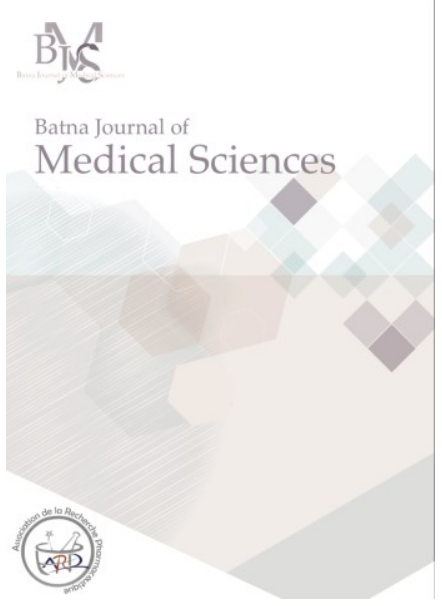




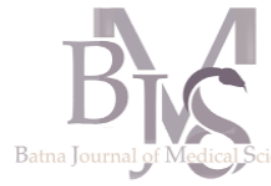

Service d'Endocrinologie et Maladies Métaboliques, $\mathrm{CHU}$ Bab El Oued, Alger - Algérie

\section{Correspondance à}

Dr. Said AZZOUG

saidazzoug@yahoo.fr

DOI : $\underline{\text { https://doi.org/10.48087/ }}$ BIMScr.2015.2117

Il s'agit d'un article en libre accès distribué selon les termes de la licence Creative Commons Attribution International License (CC BY 4.0), qui autorise une utilisation, une distribution et une reproduction sans restriction sur tout support ou format, à condition que l'auteur original et la revue soient dûment crédités.

\title{
Traitement d'une neurosarcoïdose infundibulaire par corticoïdes
}

\section{Management of infundibular neurosarcoidosis with corticosteroids}

\author{
Said Azzoug, Farida Chentli
}

\section{RÉSUMÉ}

Introduction : La neurosarcoïdose hypothalamo-hypophysaire est une pathologie rare et son traitement n'est pas bien codifié ; nous rapportons à ce propos l'observation d'une patiente qui a présenté une sarcoïdose infundibulaire infiltrant le chiasma optique et qui a répondu favorablement à la corticothérapie. Observation: Mme B.F âgée de 35 ans s'était présenté en consultation pour un syndrome aménorrhée/galactorrhée, l'examen clinique ainsi que le bilan hormonal avaient mis en évidence une insuffisance antéhypophysaire, un diabète insipide et une hyperprolactinémie modérée. L'IRM cérébrale mettait en évidence un épaississement de la tige pituitaire mesuré à $9 \mathrm{~mm}$ infiltrant le chiasma optique qui était épaissi. Le diagnostic de neurosarcoïdose a été retenu devant l'élévation de l'enzyme de conversion et le scanner thoracique qui retrouva l'aspect d'une sarcoïdose médiastinopulmonaire stade 2 . La patiente a été mise sous Prednisone à la dose de $1 \mathrm{mg} / \mathrm{kg} / \mathrm{j}$, l'évolution était marquée par une diminution de l'épaississement de la tige pituitaire et une régression de l'infiltration du chiasma optique, les lésions médiastino-pulmonaires étaient stationnaires. Sur le plan biologique, l'enzyme de conversion s'est normalisée, l'insuffisance antéhypophysaire par contre était persistante. Conclusion: Les corticoïdes peuvent être efficaces dans la sarcoïdose hypothalamohypophysaire, même s'ils ne permettent pas le plus souvent la récupération de la fonction hypophysaire, ils peuvent faire régresser les lésions et diminuer le risque de compression des structures de voisinage.

Mots clés : Sarcoïdose infundibulaire, corticothérapie, insuffisance hypophysaire, diabète insipide

\begin{abstract}
Background: hypothalamic-pituitary neurosarcoidosis is a rare disease and its management has yet to be established; we report the case of a patient presenting with an infundibular sarcoidosis that infiltrated the optic nerve and responded well to corticosteroids. Case report: Mrs. B.F, aged 35 years-old, presented with a amenorrhea and galactorrhea syndrome; physical examination and hormonal investigations have revealed pituitary insufficiency, diabetes insipidus and a moderate hyperprolactinemia. Brain MRI has shown a thickening of the pituitary stalk, measured at $9 \mathrm{~mm}$. Diagnosis of neurosarcoidosis was established with the elevation of the angiotensine converting enzyme (ACE) and a chest CT that revealed a typical grade 2 sarcoisosis. The patient underwent a treatment with Prednisone at 1 $\mathrm{mg} / \mathrm{Kg} /$ day; the evolution was favourable, with a decrease in the thickening of the pituitary stalk whereas the mediastinal and lung lesions remained stable. Lab study found a normalization of the ACE levels; however, the patient did not recover from her pituitary insufficiency. Conclusion: corticosteroids could be efficient in hypothalamic-pituitary sarcoidosis, and even if they may not allow a complete recovery of the hypothalamic function, improvement can be seen and compression may be prevented and/or limited.
\end{abstract}

Keywords: infundibular sarcoidosis, corticosteroids, pituitary insufficiency, diabetes insipidus.

\section{Pour citer l'article :}

Azzoug S, Chentli F. Traitement d'une neurosarcoïdose par corticoïdes. Batna J Med Sci 2015;2(1):75-77. https://doi.org/10.48087/ BJMScr.2015.2117

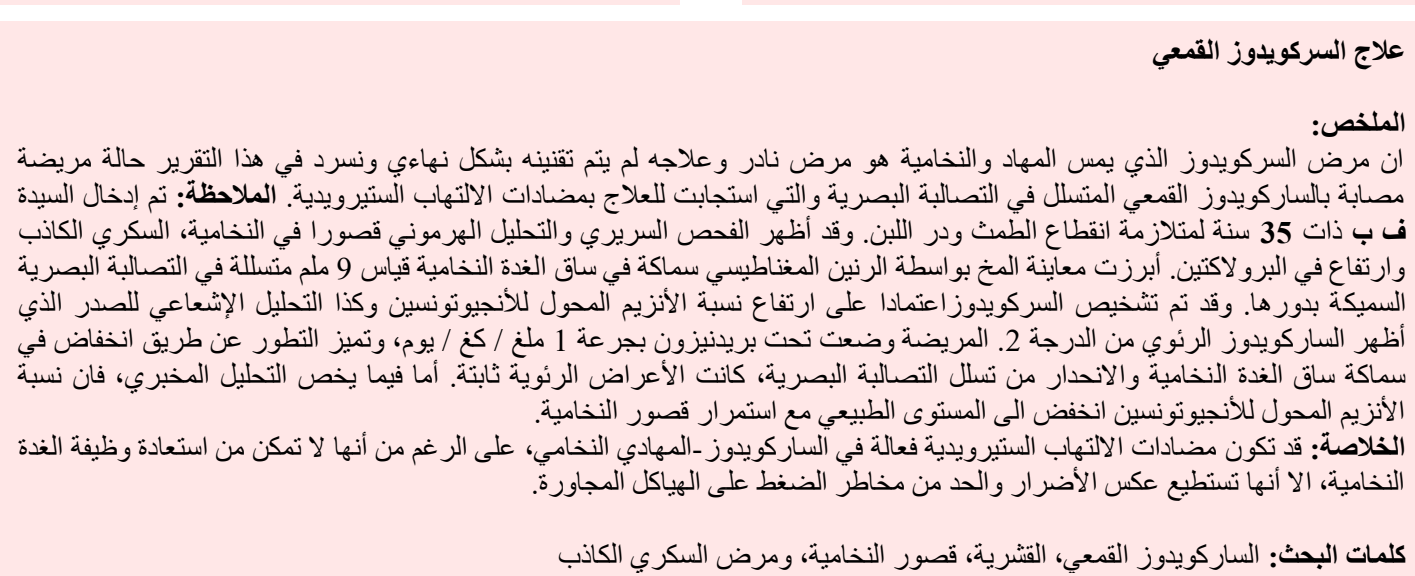




\section{INTRODUCTION}

La neurosarcoïdose hypothalamo-hypophysaire est une pathologie rare et son traitement n'est pas bien codifié ; nous rapportons à ce propos l'observation d'une patiente qui a présenté une sarcoïdose infundibulaire infiltrant le chiasma optique et qui a répondu favorablement à la corticothérapie.

\section{OBSERVATION}

Mme B.F âgée de 35 ans s'était présentée en consultation pour un syndrome aménorrhée galactorrhée. Le début de sa symptomatologie remontait à une année avant sa consultation par l'apparition d'une aménorrhée, d'une galactorrhée, d'un amaigrissement, d'une asthénie et d'une constipation. L'examen clinique mettait en évidence une patiente en normo-poids avec un indice de masse corporelle à $23 \mathrm{~kg} / \mathrm{m}^{2}$, normo-tendue à $110 / 80 \mathrm{~mm} \mathrm{Hg}$, ne présentant pas de troubles neuro-ophtalmologiques ni de symptomatologie respiratoire ou cutanée. Il existait par ailleurs un syndrome polyuropolydipsique hypotonique en rapport avec un diabète insipide central. Le bilan hormonal avait mis en évidence une insuffisance antéhypophysaire associée à une hyperprolactinémie modérée (Tableau 1).

Tableau 1: Bilan hormonal chez la patiente

\begin{tabular}{lcc}
\hline Paramètres & Patiente & Normes \\
\hline Cortisol $(\mathrm{nmol} / \mathrm{l})$ & 54 & $154-638$ \\
ACTH $(\mathrm{pg} / \mathrm{ml})$ & 28.37 & $7.2-63.3$ \\
FT4 $(\mathrm{pmol} / \mathrm{l})$ & 7.95 & $9-19$ \\
TSH $(\mu \mathrm{u} / \mathrm{ml})$ & 2.57 & $0.35-4.94$ \\
Estradiol $(\mathrm{pmol} / \mathrm{l})$ & 37 & $77-92.1$ \\
LH $(\mathrm{UI} / \mathrm{l})$ & $<0.07$ & $2.39-6.6$ \\
FSH $(\mathrm{UI} / \mathrm{l})$ & 2.82 & $3-8$ \\
GH $(\mathrm{UI} / \mathrm{l})$ & 0.47 & $0-20$ \\
Prolactine $(\mu \mathrm{U} / \mathrm{l})$ & 854 & $<785$ \\
\hline
\end{tabular}

Le bilan biologique mettait en évidence un syndrome inflammatoire avec une vitesse de sédimentation accélérée, une CRP positive et une hypergammaglobulinémie à l'électrophorèse des protéines. L'IRM cérébrale mettait en évidence un épaississement de la tige pituitaire mesuré à 9 $\mathrm{mm}$ infiltrant le chiasma optique qui était épaissi (Figure 1).

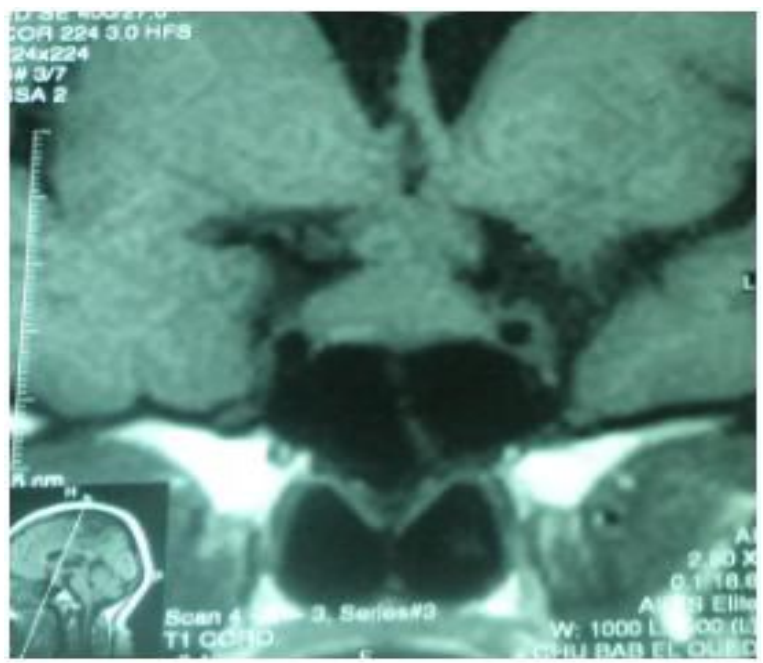

Figure 1 : épaississement du chiasma optique
Les anticorps antinucléaires étaient négatifs, l'IDR à la tuberculine retrouvait une anergie tuberculinique, l'échographie abdominale était normale. Finalement le diagnostic de neurosarcoïdose a été retenu devant l'élévation de l'enzyme de conversion et le scanner thoracique qui retrouva l'aspect d'une sarcoïdose médiastino-pulmonaire stade 2 [Figure 2].

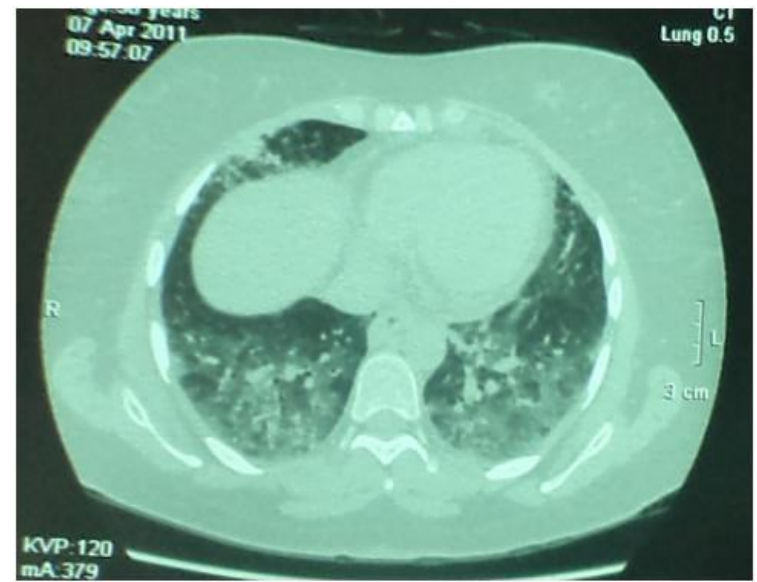

Figure 2: TDM thoraique objectivant un aspect de sarcoïdose médiastino-pulmonaire de grade 2 .

La patiente a été mise sous Prednisone à la dose de $1 \mathrm{mg} / \mathrm{kg} / \mathrm{j}$ pendant trois mois, la dose des corticoïdes a été réduite progressivement pour une durée totale du traitement de deux ans et a été arrêtée récemment. L'évolution était marquée par une diminution de l'épaississement de la tige pituitaire et une régression de l'infiltration du chiasma optique [Figure 3], les lésions médiastino-pulmonaires étaient stationnaires. Sur le plan biologique, la vitesse de sédimentation, la CRP et l'électrophorèse des protéines étaient normales. L'enzyme de conversion s'est normalisée, l'insuffisance antéhypophysaire par contre était persistante.

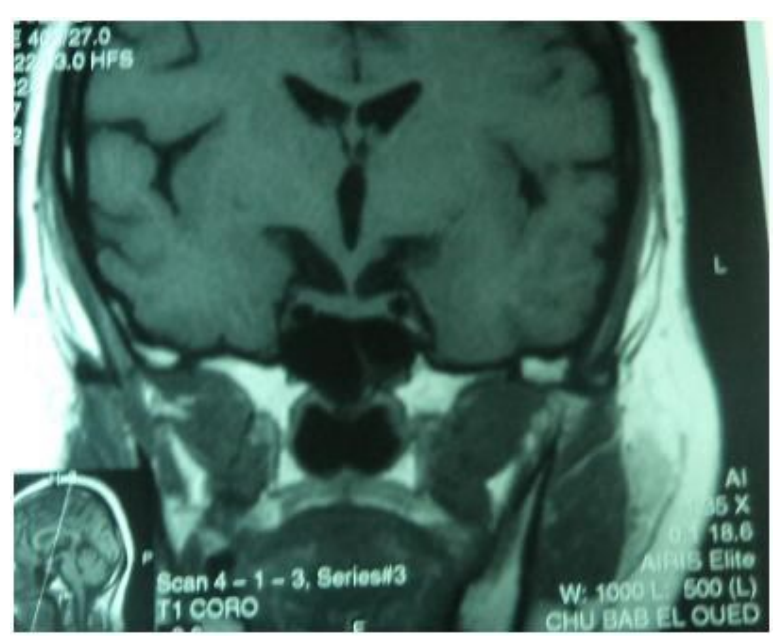

Figure 3 : régression de l'épaississement de l'infundibulum et du chiasma optique

\section{DISCUSSION}

La sarcoïdose est une pathologie chronique multi systémique caractérisée par l'accumulation de granulomes épithélioïdes sans nécrose caséeuse au niveau des organes atteints. La pathogénie de la sarcoïdose reste discutée, des facteurs génétiques, immunologiques, environnementaux et infectieux 
ont été incriminés [1]. La localisation pulmonaire est l'atteinte la plus fréquente dans la sarcoïdose, la localisation nerveuse concerne 5 à $25 \%$ des sujets [2].

La sarcoïdose hypothalamo-hypophysaire est rare représentant entre $1 \%$ et $2.5 \%$ des lésions intra-sellaires $[3,4]$. Un diabète insipide et/ou une insuffisance antéhypophysaire partielle ou globale et/ou une hyperprolactinémie sont retrouvés chez une grande proportion des patients atteints de sarcoïdose hypothalamohypophysaire comme ce fut le cas chez notre patiente. Les corticoïdes sont le principal traitement de la sarcoïdose systémique, ils sont indiqués en cas d'atteinte neurologique, cardiaque, rénale, stade II et III de l'atteinte pulmonaire, d'atteinte cutanée faciale et d'hypercalcémie [5]. Le traitement par corticoïdes entraine une amélioration des lésions radiologiques, par contre les déficits hormonaux sont le plus souvent irréversibles comme ce fut le cas chez notre patiente [6]. Rarement le traitement par corticoïdes permet de restaurer la fonction hypophysaire quand il est institué précocement [7].

\section{CONCLUSION}

Les corticoïdes peuvent être efficaces dans la sarcoïdose hypothalamo-hypophysaire, même s'ils ne permettent pas le plus souvent la récupération de la fonction hypophysaire, ils peuvent faire régresser les lésions et diminuer le risque de compression des structures de voisinage.
Déclaration d'intérêts: les auteurs ne déclarent aucun conflit d'intérêt en rapport avec cet article.

\section{RÉFÉRENCES}

1. Lannuzzi MC, Rybicky BA, Teirstein AS. Sarcoidosis. N Engl J Med 2007; 357:2153-65.

2. Lower EE, Weiss KL. Neurosarcoidosis. Clin Chest Med. 2008; 29:475-92

3. Bihan $\mathrm{H}$, Guillot $\mathrm{H}$, Fysekidis $M$, et al. Sarcoidosis: the involvement of anterior pituitary hormones is poorly recognized. Presse Med. 2012; 41(10):e524-9

4. Freda PU, Post KD. Differential diagnosis of sellar masses. Endocrinol Metab Clin North Am. 1999; 28:81-117

5. American thoracic society. Statement on sarcoidosis. Am J Respir Crit Care Med. 1999; 160:736-55

6. Langrand $\mathrm{C}$, Bihan $\mathrm{H}$, Raverot $\mathrm{G}$, et al. Hypothalamo-pituitary sarcoidosis: a multicenter study of 24 patients. Q J Med 2012; 105:981-995

7. Tanaka KI, Yamamoto M, Okazaki K, et al. Partial Improvement of Anterior Pituitary Deficiency Following Steroid Treatment in a Patient with Neurosarcoidosis Accompanied by Central Diabetes Insipidus. Intern Med 2012; 51:2175-2179

Cet article a été publié dans le « Batna Journal of Medical Sciences » BJMS, l'organe officiel de "I'association de la Recherche Pharmaceutique - Batna »

Le contenu de la Revue est ouvert "Open Access » et permet au lecteur de télécharger, d'utiliser le contenu dans un but personnel ou d'enseignement, sans demander l'autorisation de l'éditeur/auteur.

Avantages à publier dans BJMS :

- Open access : une fois publié, votre article est disponible gratuitement au téléchargement

- Soumission gratuite : pas de frais de soumission, contrairement à la plupart des revues « Open Access »

- Possibilité de publier dans 3 langues : français, anglais, arabe

- Qualité de la relecture : des relecteurs/reviewers indépendants géographiquement, respectant l'anonymat, pour garantir la neutralité et la qualité des manuscrits. 


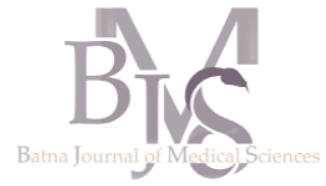

Service de psychiatrie, EHS psychiatrique El Madher, Batna - Algérie

Correspondance à :

Zoubir BENMEBAREK

zoubirbenmeb@gmail.com

DOI :https://doi.org/10.48087/ BJMScr.2015.2118

Il s'agit d'un article en libre accès distribué selon les termes de la licence Creative Commons Attribution International License (CC BY 4.0), qui autorise une utilisation, une distribution et une reproduction sans restriction sur tout support ou format, à condition que l'auteur original et la revue soient dûment crédités.

\section{Infanticide au décours d'une psychose du post-partum}

\section{Infanticide following a postpartum psychosis}

\section{Zoubir Benmebarek}

\section{RÉSUMÉ}

L'infanticide est un crime connu depuis les temps anciens. Il suscite émoi, répulsion et incompréhension car venant souvent de la personne censée la plus soucieuse du bien-être de l'enfant. Ses causes peuvent être d'origine psychopathologiques mais les contraintes socio-économiques et culturelles jouent un rôle non négligeable d'où la grande variation de ses présentations cliniques. Nous avons choisi de présenter le cas d'une femme de 26 ans, sans suivi psychiatrique antérieur à l'acte, qui a tué son enfant âgé de trois mois en le jetant pardessus la fenêtre du troisième étage de son domicile. L'examen psychiatrique a relevé des antécédents de signes anxieux non spécifiques remontant à plusieurs années avec une décompensation psychotique trois jours avant l'acte. L'approche légale de l'infanticide tend à atténuer la responsabilité de la mère au vu des modifications psycho-physiologiques et hormonales engendrées par l'accouchement. La détection précoce des troubles mentaux durant la grossesse et du post-partum et leur traitement pourrait diminuer l'incidence de ce crime.

Mots-clés : infanticide, psychose, troubles du postpartum, responsabilité atténuée

\begin{abstract}
Infanticide is a repulsive and unfathomable crime. It may be caused either by psychological disturbances or by socio economic and cultural constraints. Consequently, there are various clinical features of infanticide. We present the case of a 26 years old woman with no prior psychiatric follow-up, who killed her three months old child by throwing him over the window of the third floor. Till that day she did not show obvious signs of mental illness. The psychiatric examination showed an onset of unspecific anxiety signs many years ago with a psychotic outburst three days before the crime. Diminished responsibility is often claimed since biological and psychological changes after childbirth could trigger a vulnerability that may explain the crime. The early detection of mental disorders during pregnancy and in the postpartum period and their management could prevent and reduce the incidence of this crime.
\end{abstract}

Keywords: infanticide, psychosis, post-partum disorders, diminished responsibility

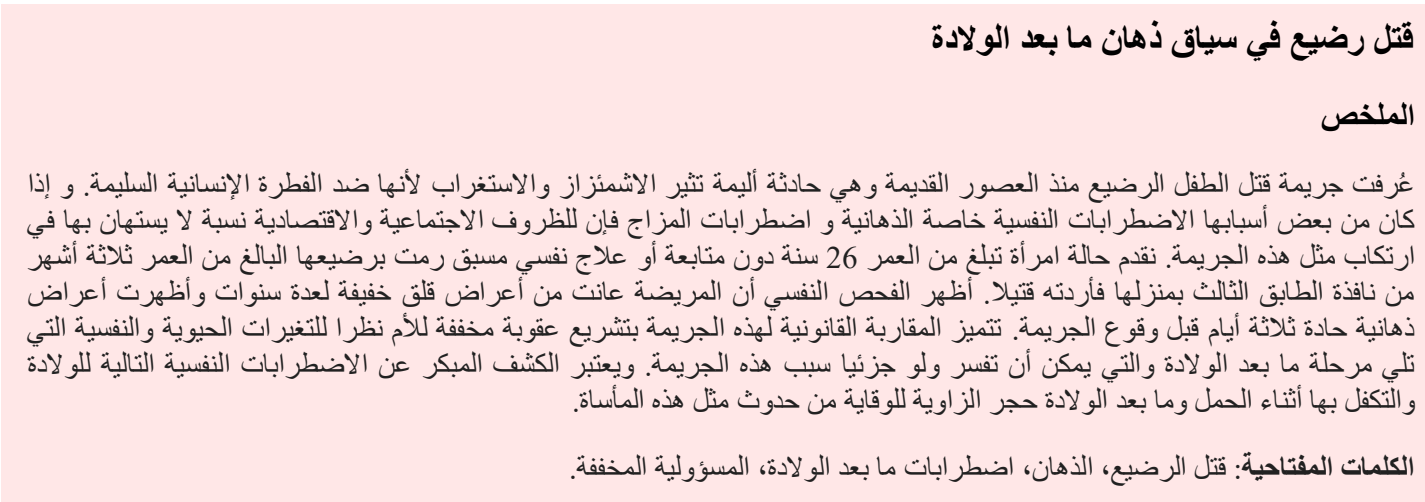

\section{INTRODUCTION}

La période puerpérale représente un risque important de déclenchement de troubles mentaux ou de décompensation d'un trouble psychiatrique préexistant surtout durant la première année après l'accouchement $[1,2]$. Les troubles mentaux vont de la dépression aux troubles anxieux et de la psychose puerpérale aux troubles thymiques bipolaires [2,3].
L'infanticide est un crime traumatisant et incompréhensible. Il soulève des interrogations sur les causes de cet acte venant souvent de la personne la plus proche, censée protéger l'enfant (la mère et à un moindre degré le père). L'infanticide d'origine psychotique suppose la présence active d'un trouble psychiatrique majeur (psychose, troubles thymiques avec caractéristiques psychotiques..) au moment de l'offense et l'absence d'un motif rational du 
crime [1]. Il est difficile de chiffrer avec exactitude l'incidence des infanticides mais il y a un accord pour dire que les chiffres officiels sont en deçà des chiffres réels tant beaucoup de cas sont cachés ou masqués [4].

\section{PRÉSENTATION DU CAS}

N. âgée de 26 ans, mariée, mère d'un enfant de 03 ans, est admise au service de psychiatrie pour infanticide d'un enfant âgé de trois mois par défenestration survenue la veille. La décision d'hospitalisation a été ordonnée par le procureur de la république au vu des troubles psychiatriques patents présentés lors de son audience.

\section{Biographie}

Issue d'un mariage non consanguin, elle était l'ainée d'une fratrie de 11 enfants. Ses parents sont vivants et bien portants, de niveau socio-économique moyen. Elle fût scolarisée jusqu'à l'âge de 10 ans puis elle a arrêté sa scolarité sur sa propre décision. Depuis son jeune âge elle disait qu'elle se voyait différente des autres, n'aimait pas les contacts sociaux, se sentait inférieure, trop timide, parlait très peu même avec ses proches parents, préférait une fois le travail de ménage terminé rester seule isolée des autres.

Dans les antécédents, dès l'adolescence on retrouve la présence de symptômes anxieux avec instabilité, irritabilité, des épisodes de dépersonnalisation passagers et des phobies non spécifiques peu intenses. Une tentative de suicide par phlébotomie a été réalisée six ans auparavant mais sans séquelles somatiques ni suivi psychiatrique.

Sa première grossesse s'est passée sans incidents majeurs sauf des moments passagers d'angoisses flottantes et des soucis exagérés. Elle a accouché par voie basse et a allaité son bébé jusqu'à l'âge de deux ans. Elle a eu une bonne relation avec l'enfant. Lors de la deuxième grossesse, elle a aussi présenté des angoisses intermittentes, se sentait lasse, fatiguée, communiquait très peu avec son entourage. Elle n'a ni consulté ni pris aucun traitement pour ces symptômes. L'accouchement se déroula sans problèmes, elle n'a pas présenté de signes particuliers durant les semaines suivant son accouchement.

Trois jours avant l'incident, s'installa une insomnie et une anorexie puis apparition brutale d'une activité hallucinatoire et délirante centrée sur l'enfant ; elle était submergée d'idées telles : « je n'ai pas eu un enfant», entendait des voix qui lui disaient «si tu jettes l'enfant tu vas guérir». Elle avait l'impression que les murs lui parlaient. L'intensité de ces symptômes va croître, L'idée de se débarrasser de l'enfant était toujours prégnante; les hallucinations impératives l'accablaient continuellement.

Le troisième jour elle n'a pas donné à l'enfant sa tétée ; le soir, sur insistance du mari qui lui demandait de le nourrir, elle est partie vers sa chambre, la fenêtre était ouverte, elle a froidement jeté l'enfant du 3ème étage. Elle est restée calme, n'a pas crié ou pleuré. Elle n'a pas bougé de la chambre. À Son mari qui lui demandait où est l'enfant elle lui répondait qu'elle n'a jamais eu d'enfant.

Après la découverte macabre la patiente fut ligotée, on appela la police et elle fut transportée à l'hôpital local d'où elle fût orientée vers notre service sur ordre du procureur de la république.

\section{L'examen à l'admission}

À l'admission, l'examen psychiatrique retrouve une patiente calme, indifférente, contact froid, elle était envahie par une activité délirante et hallucinatoire, présentait une incohérence de la pensée, un déni de l'accouchement et de la naissance de l'enfant.

L'examen psychologique retrouve une absence d'autocritique, absence de sentiment de culpabilité et révèle une désorganisation idéique, des troubles de la mémoire et un déni des troubles. Elle disait qu'elle ne voulait pas cet enfant et qu'elle ne pouvait assumer sa responsabilité.

\section{Évolution sous traitement}

Une neuroleptisation est entamée dès l'admission. Une évolution favorable est observée après trois semaines de traitement neuroleptique classique. Elle autocritique acte, décrit les symptômes qu'elle a présentés avec détails, reconnaît qu'elle était malade sous l'emprise de pensées bizarres et d'hallucinations auxquelles elle n'a pu échapper ou résister.

L'hospitalisation de la patiente dura six mois et neuf jours. La sortie fût décidée par le procureur de la république suite à un certificat attestant l'amélioration clinique. Elle regagna son domicile vu qu'elle fût considérée irresponsable de ses actes au moment des faits.

\section{Discussion diagnostique}

Devant ce tableau clinique un trouble dépressif du postpartum avec caractéristiques psychotiques est éliminé car elle n'a pas présenté des signes dépressifs francs bien que l'asthénie, le sentiment d'incapacité peuvent faire penser à ce diagnostic. Le caractère aigu de l'apparition des troubles, l'absence d'humeur dépressive, d'idées suicidaires, écartent ce diagnostic. La psychose secondaire à une affection organique est écartée devant l'absence des signes somatiques, la normalité des bilans et une TDM cérébrale sans anomalies.

Le diagnostic retenu (selon le DSM IV) est un trouble psychotique bref avec début lors du post-partum.

\section{DISCUSSION}

Il y a plusieurs termes définissant le meurtre d'un enfant qui sont interchangeables; l'infanticide définit le meurtre d'un enfant âgé de plus de 1 jour et de moins d'un an et le néonaticide le meurtre d'un enfant âgé de moins d'un jour. Le filicide, lui englobe tout meurtre d'un enfant âgé de plus d'un jour et de moins de 18 ans et le libéricide tout meurtre d'un enfant âgé de plus d'un an et moins de 18 ans $[5,6]$.

L'infanticide est un acte qui renvoie à des causes multiples, sociales, culturelles, économiques ou psychiques bien que la faible fréquence des troubles mentaux dans l'infanticide est plutôt la règle [5,7-10]. Certains auteurs font une distinction entre le néonaticide où les facteurs socio-économiques jouent un rôle important (niveau socio-économique bas, célibat, jeune âge, grossesse non désirée etc.) et l'infanticide où ce 
sont les facteurs psychiatriques qui prédominent [4-6].

\section{Aspects descriptifs de l'infanticide}

- Les méthodes de l'infanticide sont variables : la suffocation, la strangulation, la noyade, l'exposition aux intempéries les traumatismes crâniens, les coups y compris les coups de couteaux [5] ;

- La victime : certains auteurs considèrent que les garçons présentent plus de risque que les filles d'être victime d'infanticide [9] ;

- Profil de la mère: le pic de prévalence des mères infanticides se situe entre 20 et 25 ans, de milieu socio culturel modeste et sans profession. Il est observé chez ces femmes une immaturité, des difficultés à communiquer, une grande solitude affective, le déni d'une grossesse souvent cachée à l'entourage et l'illégitimité de l'enfant [8] .

\section{Infanticide et troubles mentaux}

La période post natale est une période de vulnérabilité au vu des perturbations importantes biologiques et psychologiques générées par l'accouchement. Les femmes en suites de couche sont sujettes à de nombreuses affections psychiatriques anxieuses, thymiques ou psychotiques [2,3]. L'incidence des troubles psychotiques chez les mères ayant commis un infanticide varie selon les études de 10 à $66 \%$ [5,6,11]. Cette différence est expliquée par la variété des échantillons et par l'âge des enfants victimes d'infanticides.

Dans l'étude D'Orban, une population de 89 femmes accusées d'avoir tué ou tenté de tuer leurs enfants est étudiée durant la période 1970-1975 en Angleterre. Sur ce total, 14 femmes uniquement sont psychotiques [12]. Menik, en analysant des expertises pour infanticide, a retrouvé uniquement 3\% de pathologies mentales alors que 97 \% étaient déclarées saines au moment de l'acte [10]. Adinkrah a analysé 16 infanticides sur une période de 11 ans au Fiji ; aucun n'était secondaire à une maladie mentale [7]. La synthèse faite par Resnick retrouve seulement $17 \%$ de psychotiques parmi les femmes ayant commis un néonaticide alors que $66 \%$ des mères filicides étaient psychotiques [5]. Enfin, l'incidence des troubles psychotiques chez les mères ayant commis un infanticide en Autriche et en Finlande entre 1995 et 2005 était de $10 \%$ et de $12 \%$ respectivement [11].

\section{Aspects cliniques des troubles psychiatriques}

Malgré les descriptions cliniques détaillés et anciennes des psychoses du post-partum (ou psychoses puerpérales), cette entité n'est pas reconnue comme un trouble psychiatrique indépendant [13]. En effet, le DSM IV ( Manuel diagnostique et statistique des troubles mentaux) ne donne aucune spécification aux troubles mentaux survenant durant cette période excepté leur caractère temporal c'est-à-dire survenant dans les quatre premières semaines après l'accouchement [14], alors que certains cliniciens prolongent cette période jusqu' à une année [6].

Cliniquement, la psychose se traduit par des troubles perceptifs, un syndrome pseudo confusionnel, une labilité affective et une désorganisation cognitive [13]. Les troubles sont fluctuants et changeant, avec des phases de stabilité et des périodes de désorganisation comportementale [13].

L'infanticide suite à un trouble psychotique du post-partum est caractérisé par des signes précurseurs anxieux, par l'imprévisibilité de l'acte, la présence de troubles délirants et hallucinatoires, le déni de l'enfant ainsi que l'indifférence aux conséquences de l'infanticide [15].

Le lien entre pathologie mentale apparue après l'accouchement et l'infanticide n'est pas clairement établi. Si certains auteurs y voient, dans les modifications psychologiques et biologiques durant la première année suivant l'accouchement, des facteurs déclenchant de troubles psychotiques spécifiques qui entrainent dans leurs sillages l'infanticide (survenue après l'accouchement, rôle hormonal et neuro endocrinien, prédominance des troubles affectifs, présence de signes psychotiques et confusionnels) et l'assimilent à une psychose organique $[4,13]$; d'autres y voient seulement un phénomène purement chronologique et temporel, l'infanticide étant considéré comme tout autre crime dont la parturience n'est qu'un facteur précipitant [1].

\section{Aspect légal de l'infanticide}

Certaines pays (Angleterre, Australie, Canada, Turquie, Inde etc.) prenant en compte l'état psychologique particulier du post-partum et de l'allaitement, ont légiféré dans le sens de l'allègement des peines (l'infanticide est considéré alors comme un homicide involontaire) justifiant l'approche médicale de prévention et de réhabilitation au lieu de la punition $[5,16]$ alors que d'autre pays (Ecosse, Etats-Unis, France) jugent les mères coupables comme n'importe quel autre crime et sont soumises à la réglementation concernant tout homicide (qu'il soit associé ou non à une maladie mentale) $[1,13,17]$.

\section{L'infanticide dans la loi algérienne}

Selon l'article 259 du code pénal, l'infanticide est le meurtre ou l'assassinat d'un nouveau né.

Selon l'article 261, tout coupable de d'assassinat ou de parricide ou d'empoisonnement est puni de mort. Toutefois, la mère, auteur principal ou complice de l'assassinat ou du meurtre de son enfant est punie de la réclusion à temps, de dix à vingt ans, mais sans que cette disposition puisse s'appliquer à ses coauteurs ou complices [18].

La tendance du législateur est donc de prendre en considération l'aspect particulier de l'infanticide et les modifications psycho-physiologiques des suites de couche en commuant la peine prévue pour une mère supposée saine. En cas de trouble mental sévère, la non responsabilité est la règle selon l'article $47 \mathrm{du}$ code pénal et l'internement judiciaire dans un servie fermé sera ordonné conformément à l'article 21 dudit code [18].

\section{Prévention}

L'identification des troubles mentaux durant la grossesse, et le suivi des femmes à risque représentent des mesures indispensables dans la prévention de l'infanticide $[4,6]$.

Le rôle du psychiatre est important dans le dépistage, la détection et la prise en charge à temps de ces troubles [6]. La période anténatale est le temps idéal pour évaluer en détail l'état psychologique de la femme enceinte (antécédents psychiatriques, antécédents familiaux, symptômes présentés, caractère et personnalité, facteurs sociaux etc.) et le suivi sera 
prolongé après l'accouchement, surtout durant le premier trimestre [13].

L'examen sera centré, en plus des symptômes psychiques, sur l'attitude de la mère envers son enfant. Les idées de nuire à l'enfant doivent être explorées systématiquement (idées délirantes, enfant non désiré etc.) [6]. Les dépressions et les psychoses du post-partum sont les pathologies mentales les plus à même de provoquer un infanticide [4].

Ceci se fera sans oublier que la variabilité et la fluctuation des symptômes, leur non spécificité, l'imprévisibilité de l'acte peuvent rendre la prévention difficile d'autant plus que beaucoup des cas d'infanticide surviennent chez des patientes non suivis [5].

\section{CONCLUSION}

L'infanticide est un crime multifactoriel qui doit être analysé dans son contexte. S'il est secondaire à une affection psychiatrique il doit être pris en charge dans un cadre médical pluridisciplinaire plutôt que dans un cadre légal. La majorité des cas d'infanticides sont imprévisibles mais l'identification des femmes enceintes à risque et le développement d'un programme de suivi rapproché pourrait diminuer l'incidence de ce drame.

Déclaration d'intérêts : l'auteur ne déclare aucun conflit d'intérêt en rapport avec cet article.

\section{RÉFÉRENCES}

1. Lambie I Mothers who kill. The crime of infanticide. International Journal of Law and Psychiatry $2001 ; 24: 71-80$.
2. Sutter A-L, Lacaze I, Loustau N, Paulais J-Y, Glatigny-Dallay E.Troubles psychiatriques et période périnatale. Annales médico psychologiques $2005 ; 163$ : 524-528.

3. Brockington I. Diagnosis and management of post-partum disorders: a review. World Psychiatry June 2004 ; 3:2 :89-95.

4. Craig M. Perinatal risk factors for neonaticide and infant homicide: can we identify those at risk ? J R soc Med 2004; 97:57-61.

5. Resnick Philipe et al, Meurtre de nouveau-né : une synthèse psychiatrique sur le néonaticide. Enfance \& Psy 2009/3 ; 44 :42-54.

6. West SG. An overview of filicide. Psychiatry (Edgmont). $2007 ; 4(2)$ : 48-57.

7. Adinkrah M. Maternal infanticides in Fiji. Child Abuse \& Neglect $2000 ; 24,12: 1543-1555$

8. Bénézech $M$, Le Bihan $P$ et Bourgeois $M L$. Criminologie et psychiatrie. Encycl Méd Chir, Psychiatrie, 37-906-A-10, 2002, 15 p.

9. Marks M. Infanticide. Psychiatry 2006 ; 5:1: 13-15.

10. Menick D M. Les contours psychosociaux de l'infanticide en Afrique noire: le cas du Sénégal. Child Abuse \& Neglect 2000 ; 24,12 : 15571565.

11. Putkonen $\mathrm{H}$, Amon $\mathrm{S}$, Almiron $\mathrm{MP}$, et al. Filicide in Austria and Finland - A register-based study on all filicide cases in Austria and Finland 1995-2005. BMC Psychiatry 2009 ; 9:74

12. D'Orbán PT. Women who kill their children. Br J Psychiatry 1979 ; 134:560-71.

13. Spinelli MG. Maternal infanticide associated with mental illness: prevention and the promise of saved lives. Am J Psychiatry 2004 ; 161:1548-1557.

14. American Psychiatric Association. Manuel diagnostique et statistique des troubles mentaux IV. Paris :Masson ;1996.

15. Rammouz I, D.A. Tahiri, R. Aalouane, S. Kjiri, A. Belhous, J.E. Ktiouet, F.Z. Sekkat. Infanticide en post-partum: à propos d'un cas. L’Encéphale (2008) ; 34 : 284-288.

16. Dean PJ. Child homicide and Infanticide in New Zealand. International Journal of Law and Psychiatry $2004 ; 27$ : 339-348.

17. Getti JP. Un traitement judiciaire de I'infanticide. Perspectives Psy $2007 / 2 ; 46: 115-118$.

18. Didane M. Code penal. Alger : Belkeise edition; 2012.

Cet article a été publié dans le « Batna Journal of Medical Sciences » BJMS, l'organe officiel de "I'association de la Recherche Pharmaceutique - Batna »

Le contenu de la Revue est ouvert « Open Access » et permet au lecteur de télécharger, d'utiliser le contenu dans un but personnel ou d'enseignement, sans demander l'autorisation de l'éditeur/auteur.

Avantages à publier dans BJMS :

- Open access : une fois publié, votre article est disponible gratuitement au téléchargement

- Soumission gratuite : pas de frais de soumission, contrairement à la plupart des revues «Open Access »

Possibilité de publier dans 3 langues : français, anglais, arabe

- Qualité de la relecture : des relecteurs/reviewers indépendants géographiquement, respectant l'anonymat, pour garantir la neutralité et la qualité des manuscrits.

Pour plus d'informations, contacter BatnaJMS@gmail.com

ou connectez-vous sur le site de la revue : www.batnajms.com 


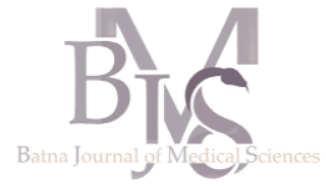

${ }^{1}$ Service de parasitologie mycologie CHU de Batna, Batna - Algérie.

2 Service d'orthopédie CHU de Batna, Batna - Algérie.

\section{Correspondance à :}

Ouanassa HAMOUDA

hamoudaouanassa@yahoo.fr

DOI :https://doi.org/10.48087 BJMScr.2015.2119

\section{Il s'agit d'un article en libre} accès distribué selon les termes de la licence Creative Commons Attribution International License (CC BY 4.0), qui autorise une utilisation, une distribution et une reproduction sans restriction sur tout support ou format, à condition que l'auteur original et la revue soient dûment crédités.

\title{
Kyste hydatique du psoas: à propos d'un cas
}

\author{
Hydatid cyst of the psoas: a case report.
}

\section{Ouanassa Hamouda ${ }^{1}$, Mayssa Benchikh ${ }^{1}$, Nouar Boudjouraf ${ }^{2}$}

\section{RÉSUMÉ}

Nous rapportons un cas rare de kyste hydatique du psoas chez une patiente âgée de 79 ans, qui a présenté une tuméfaction de la face antéro-interne de la cuisse droite. L'échographie abdomino-pelvienne, la tomodensitométrie et la sérologie hydatique ont contribué au diagnostic préopératoire. Le traitement était chirurgical et a consisté en une périkystectomie totale par un abord retropéritonéal. La masse a été enlevée en totalité par une approche rétropéritonéale.

Mots-clés : Kyste hydatique, périkystectomie, psoas.

\begin{abstract}
We report a rare case of hydatid cyst of the psoas in a 79-year-old woman, who had a swelling on the internal anterior surface of her right thigh. The abdominal and pelvic ultrasound, computed tomography and hydatid serology contributed to the preoperative diagnosis of a hydatid cyst. Treatment was surgical; a total peri-cystectomy was performed, the cyst was completely removed based on a retroperitoneal approach.
\end{abstract}

Keywords: hydatid cyst, pericystectomy; psoas.

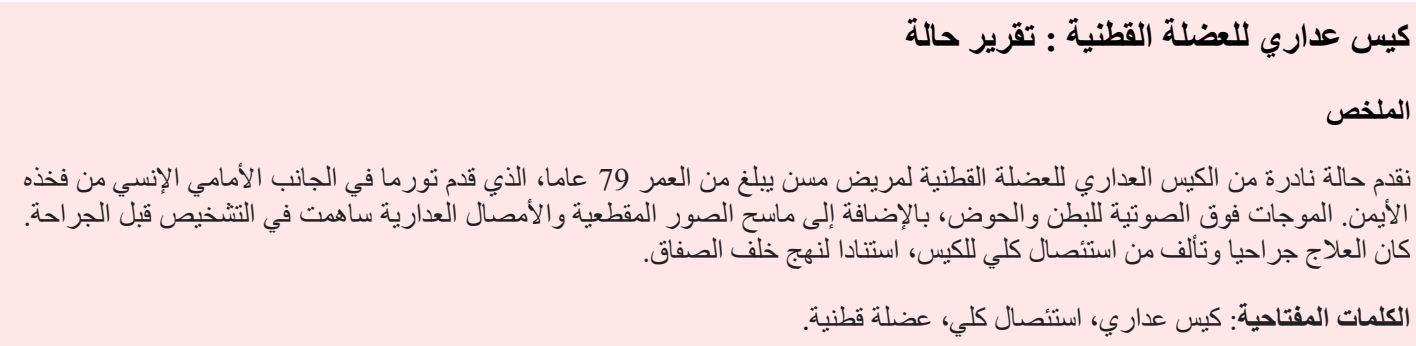

\section{INTRODUCTION}

L'hydatidose est une anthropozoonose cosmopolite commune à l'homme et à de nombreux mammifères due au développement dans l'organisme d'un Taenia du chien appelé Echinococcus granulosis [1]. Elle se manifeste typiquement au niveau hépatique ou pulmonaire; cependant une fois sur dix, l'embryon va se loger dans n'importe quel tissu ou organe du corps humain [2]. La localisation musculaire isolée du kyste hydatique est une entité rare. Un peu plus d'une trentaine de cas sont rapportés dans la littérature mondiale concernant le kyste hydatique du muscle psoas [3].

Le but de cet article est de rapporter une nouvelle observation de kyste hydatique du psoas; le diagnostic a été fait avant l'intervention grâce à l'échographie, à la Tomodensitométrie et à la sérologie hydatique.

\section{OBSERVATION}

Mme D.Y âgée de 79 ans d'origine rurale, sans antécédents pathologiques particuliers, a été hospitalisée dans le service d'orthopédie pour exploration d'une tuméfaction de la face antéro-interne de la cuisse droite apparue de façon progressive et insidieuse depuis 3 mois. La patiente a été en bon état général, elle n'a rapporté aucun déficit sensitivomoteur en particulier au membre inférieur droit. L'examen clinique de l'appareil locomoteur objectivait une tuméfaction au niveau de la partie proximale de la face antéro-interne de la cuisse droite, ferme, indolore, mobile à la palpation, sans signe inflammatoire, de contour régulier, mesurant 20 centimètres de grand axe.

La radiographie du fémur droit numérisé (figure 1) ainsi que l'échographie des parties molles de la cuisse droite ont objectivé une volumineuse collection liquidienne pariétale de la racine de la cuisse étendue au niveau de la loge du psoas de plus de 20 centimètres. Il n'y avait pas de lésion osseuse.

Une échographie abdomino-pelvienne avait montré une distension de la bourse ilio-psoas droite (figure 2). Le reste de l'exploration de la cavité abdominale ne retrouvait aucune lésion 
identique ni dans le foie, ni dans la rate, ni dans le rein. La radiographie du thorax ne montrait pas d'opacités sur les champs pulmonaires.

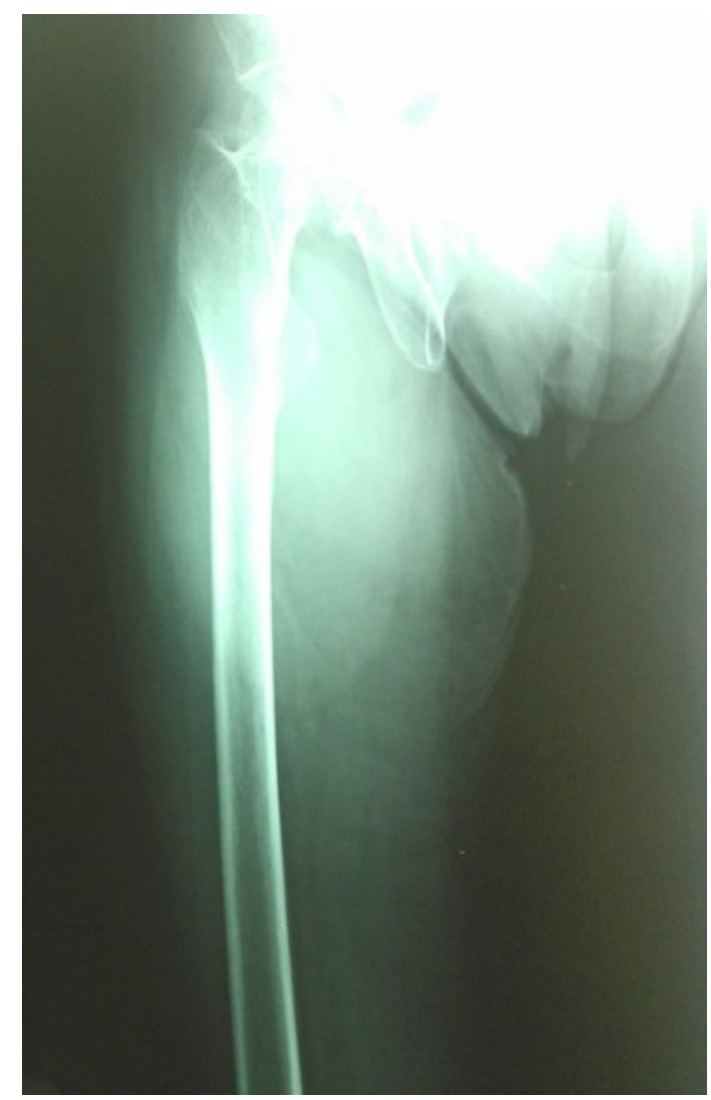

Figure 1. Radiographie numérisée du fémur droit. Volumineuse collection liquidienne pariétale de la racine de la cuisse droite étendue au niveau de la loge du psoas, mesurant plus de $20 \mathrm{~cm}$.

La tomodensitométrie abdomino-pelvienne et fémorale montrait une volumineuse collection liquidienne du psoas droit étendue via le canal inguinal vers la racine de la cuisse de plus de 20 centimètres de diamètre (figure 3 ).

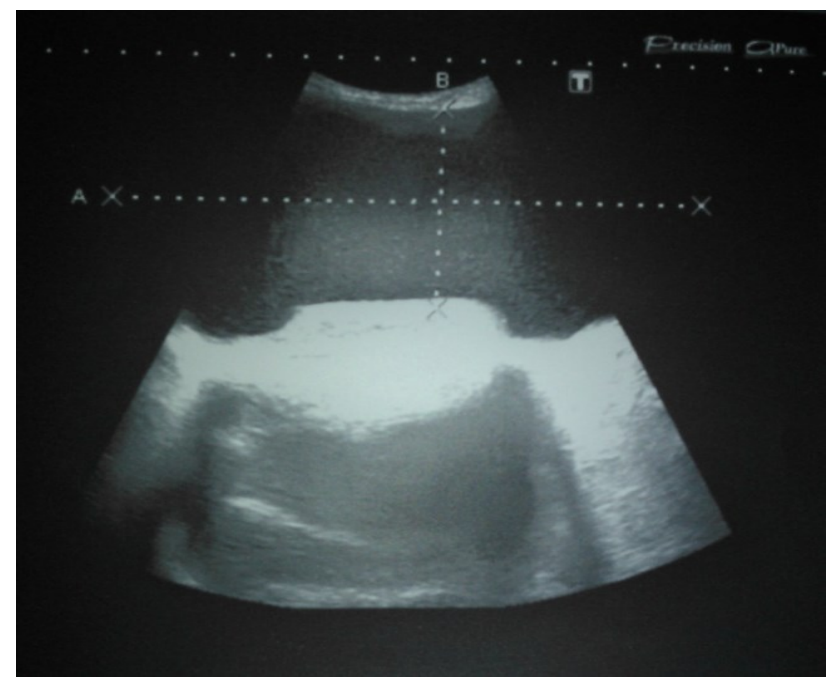

Figure 2. Échographie abdomino-pelvienne montrant une distension de la bourse ilio-psoas droite.
La sérologie hydatique par test Elisa (enzyme linked immunosorbent assay) était positive avec un taux de 23,70 UI/l. Devant le contexte épidémiologique et l'aspect très évocateur de la lésion kystique à l'échographie, le diagnostic de kyste hydatique a été retenu. Le traitement était chirurgical et consistait en une périkystectomie totale par un abord rétropéritonéal. La masse a été enlevée en totalité.

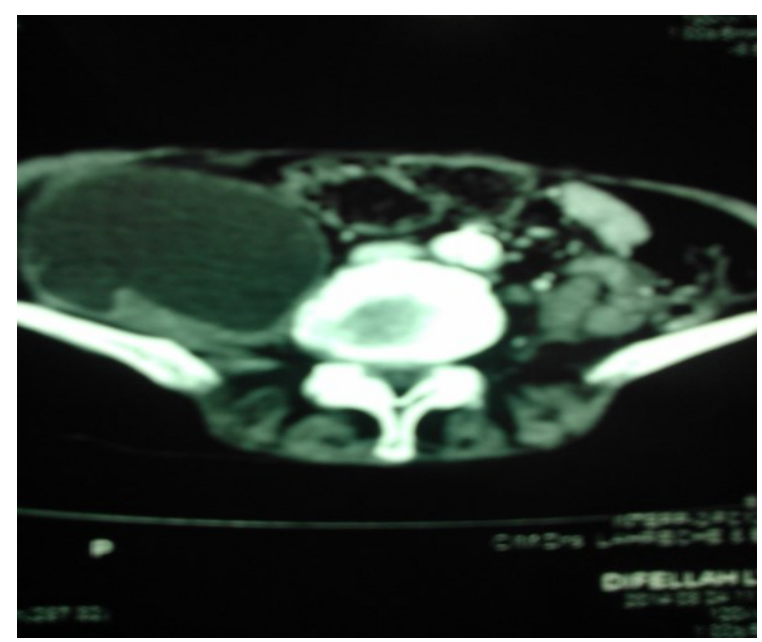

Figure 3. Tomodensitométrie abdomino-pelvienne et fémorale ayant montré une volumineuse collection liquidienne du psoas droit étendue via le canal inguinal.

\section{DISCUSSION}

Les localisations hépatiques et pulmonaires représentent $90 \%$ de toutes les localisations du kyste hydatique. La localisation hydatique musculaire est rare (1-3\%), même en pays d'endémie. Les muscles proximaux des membres inférieurs sont le plus fréquemment touchés [5]. Les kystes hydatiques primaires développés dans le tissu rétropéritonéal, sans connexion apparente, demeurent une entité exceptionnelle. Les oeufs du ténia échinocoque ingérés par l'homme libèrent dans l'intestin l'embryon hexacanthe. Ce dernier franchit la muqueuse intestinale et passe dans la circulation porte. Le courant portal emporte cet embryon vers le premier barrage qui est le foie où il s'arrête, sinon par l'intermédiaire des veines sus-hépatiques, le parasite gagne la veine cave inférieure, le coeur droit, puis le poumon qui constitue le $2^{\text {ème }}$ barrage. Une fois sur dix, l'embryon hexacanthe franchit les deux barrages, se retrouve dans la grande circulation et peut se loger dans n'importe quel endroit de l'organisme [6]. Sur le plan pathogénique, la présence d'une localisation musculaire implique que l'embryon hexacanthe avait franchi le filtre hépatopulmonaire pour se fixer sur un organe, le plus souvent bien irrigué, le long de son trajet de migration [7]. L'atteinte de régions moins bien vascularisées est expliquée par certains auteurs par un mode de contamination direct, secondaire à la morsure d'un animal infesté ou à la souillure d'une plaie par les défécations d'animaux infectés [8].

Le diagnostic de kyste hydatique du muscle psoas, souvent difficile, est celui d'une masse abdominale, à localisation iliaque ou lombaire, rénitente, fixée au plan profond. Certains kystes peuvent être révélés par des complications à type de compression nerveuse, urinaire, vasculaire, ou par une surinfection par voie hématogène pouvant engendrer un sepsis parfois sévère. Le motif de consultation est une 
tuméfaction des parties molles augmentant progressivement de volume. L'échographie permet d'évoquer le diagnostic devant l'échostructure liquidienne dans un contexte épidémique, et elle permet d'éviter la ponction. Le muscle psoas est une localisation exceptionnelle [6]. Le diagnostic différentiel se fait avec toutes les tumeurs kystiques ou mixtes rétro-péritonéales, les abcès à pyogènes ou les abcès tuberculeux [9].

Sur le plan thérapeutique, la chirurgie reste la méthode de référence et consiste en une périkystectomie totale par un abord extra-péritonéal mais elle reste dangereuse du fait des rapports intimes du kyste avec l'uretère ou le pédicule iliaque [9].

\section{CONSLUSION}

Dans les pays d'endémie hydatique, toutes les localisations du kyste hydatique sont possibles. La localisation au muscle psoas est rare et le diagnostic peut être confirmé par l'échographie et la Tomodensitométrie. Le traitement consiste en une périkystectomie totale par un abord extra péritonéal. La recherche d'autres localisations des kystes est impérative avant toute décision thérapeutique.

Déclaration d'intérêts : les auteurs ne déclarent aucun conflit d'intérêt en rapport avec cet article.

\section{RÉFÉRENCES}

1. Sakka SA. Kyste hydatique primitif de la cuisse à manifestation douloureuse au niveau de la hanche a propos d'un cas. Rev Chir Orthop Reparatrice App Mot 1993;79:226-8.

2. Venara A, Mehinto DK, Lermite E, Chabasse D, Hamy A, Arnaud JP. Localisations primitives inhabituelles du kyste hydatique. La presse medicale 2011;40:438-42

3. El Malki HO, El Mejdoubi Y, Bouslami B, Mohsine R, Ifrine L, Belkouchi A. Kyste hydatique du muscle psoas. Cahiers santé 2007;17:177-79

4. Soufi M, Lahlou MK, Messrouri R, et al. Kyste hydatique du psoas : à propos de deux cas. Journal de Radiologie 2010;91:1292-4.

5. Aubry P. Hydatidose ou Kyste hydatique. médecine tropicale. Mise à jour 06/11/2013

6. Louchi A, Chtata $H$, Araoui M, Zizi A. Kyste hydatique du muscle psoas. Ann chir 2001;126:820-1

7. Meddeb N, Bachrouch N, Elleuch M. Kyste hydatique des adducteurs de la cuisse. Aspect I.R.M, à propos d'un cas. Bull Soc Pathol Exot, 2001, 94, 2, 106-108

8. Gignoux M, Caltran M. A propos d'un cas de kyste hydatique du creux poplité. Lyon Chir, 1969;65:905-7

9. Benchekroun A, Jira H, Cheikhani OJ, Kasmaoui EH, Zannoud M, Faik M. Kyste hydatique du psoas. À propos d'un cas. Ann Urol 2001;35:108-10

Cet article a été publié dans le « Batna Journal of Medical Sciences » BJMS, l'organe officiel de "I'association de la Recherche Pharmaceutique - Batna »

Le contenu de la Revue est ouvert « Open Access » et permet au lecteur de télécharger, d'utiliser le contenu dans un but personnel ou d'enseignement, sans demander l'autorisation de l'éditeur/auteur.

Avantages à publier dans BJMS :

- Open access : une fois publié, votre article est disponible gratuitement au téléchargement

- Soumission gratuite : pas de frais de soumission, contrairement à la plupart des revues « Open Access »

- Possibilité de publier dans 3 langues : français, anglais, arabe

- Qualité de la relecture : des relecteurs/reviewers indépendants géographiquement, respectant l'anonymat, pour garantir la neutralité et la qualité des manuscrits.

Pour plus d'informations, contacter BatnaJMS@gmail.com

ou connectez-vous sur le site de la revue : www.batnajms.com 


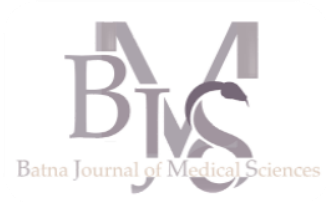

\title{
Endométriose colique : à propos de 2 cas
}

\section{Colic endometriosis: report of 2 cases.}

\author{
Wissem Merzouki ${ }^{1}$, Saida Khouni ${ }^{1}$, Ali Benazza ${ }^{2}$, Omar Bafdel ${ }^{2}$, Aissa Hadj \\ Brahim $^{3}$, Sihem Guerfi ${ }^{4}$, Hachani Khadraoui ${ }^{4}$
}

${ }^{1}$ Unité d'endoscopie digestive, CHU Batna, Algérie

${ }^{2}$ Service de Chirurgie Générale, CHU Batna, Algérie

${ }^{3}$ Service de radiologie, EPH El Meniaa, Ghardaia, Algérie

${ }^{4}$ Service d'anatomopathologie, CHU Batna, Algérie

\section{Correspondance à}

Wissem MERZOUKI

wissemerzouki@yahoo.fr

DOI :https://doi.org/10.48087/ BJMScr.2015.2120

Il s'agit d'un article en libre accès distribué selon les termes de la licence Creative Commons Attribution International License (CC BY 4.0), qui autorise une utilisation, une distribution et une reproduction sans restriction sur tout support ou format, à condition que l'auteur original et la revue soient dûment crédités.

\section{RÉSUMÉ}

L'endométriose est une affection gynécologique touchant 5 à $10 \%$ des femmes en activité génitale. La localisation intestinale est la forme extra pelvienne la plus fréquente de l'endométriose ; son diagnostic reste difficile et peut même simuler, dans certains cas, un cancer $\mathrm{du}$ colon. Nous rapportons 2 observations cliniques d'endométriose colique, la première à localisation rare caecoappendiculaire suspecte de malignité et la deuxième de siège rectal, chez 2 jeunes femmes mariées en pleine activité génitale dont une est nullipare. Dans les 2 cas, la découverte de la masse endométriotique a été radiologique (TDM abdomino-pelvienne, IRM pelvienne) réalisée à la suite de manifestations cliniques assez variables (douleurs pelviennes chroniques, dyschésie rectale). L'atteinte digestive est dans les 2 cas associée à une atteinte gynécologique ; la coloscopie a été normale chez la première patiente ; par contre elle a mis en évidence chez la deuxième patiente au 5 ème jour du cycle, une rectite congestive avec un aspect bombé correspondant à la compression de l'endométriose de la cloison recto-vaginale. Les 2 patientes ont reçu un traitement hormonal (contraceptif) pendant une durée de 3 à 6 mois avec un contrôle gynécologique; par contre la patiente chez qui on a découvert une masse cœco-appendiculaire suspecte, a bénéficié d'un traitement chirurgical radical. L'évolution sous traitement a été favorable chez les 2 patientes.

Mots clés : Endométriose colique, rectite, traitement chirurgical

\begin{abstract}
Endometriosis is a gynaecological condition affecting 5 to $10 \%$ of genitally active women. Intestinal involvement is the most frequent extra-pelvic form; its diagnosis remains difficult and can even simulate colic cancer. We report here 2 cases of colic endometriosis; the first is a rare location on the caecum and appendix, which was suspect for malignancy, and the second was located on the rectum, in two married women, one being nulliparous. In both cases, diagnosis of the mass was established based on imaging findings (abdominal and pelvic CT-scan, pelvic MRI), which were performed after variable symptoms (chronic pelvic pain, rectal dyschezia). In both cases, gynaecological manifestations were associated with digestive complaints. In patient 1 , colonoscopy was normal, but in patient 2 , rectoscopy performed at the $5^{\text {th }}$ day of the menstrual cycle showed congestive proctitis with a bulged appearance, corresponding to the compression of endometriosis over the recto-vaginal bulkhead. Both patients received a hormonal treatment (contraceptive therapy) during a period of 3 to 6 months, with gynaecological follow-up; however, in the patient in whom a suspect caeco-appendicular lesion has been discovered, surgical removal was performed. Evolution was favourable under treatment in both patients.
\end{abstract}

Keywords: Colic endometriosis, proctitis, surgical treatment.
Pour citer l'article :

Merzouki W, Khouni S, Benazza A, et al.

Endométriose colique : à propos de 2 cas. Batna J

Med Sci 2015;2(1):85-88.

https://doi.org/10.48087/

BJMScr.2015.2120

\section{سرد حالتين للالتهاب بطانة الرحم القولونية}

ملفصل بطانة الرحم هو من الأمر اض التي تصيب 5-10٪ من النساء في سن الإنجاب. التوطين في الأمعاء (القولون) هو النوع الأكثر شيوعا

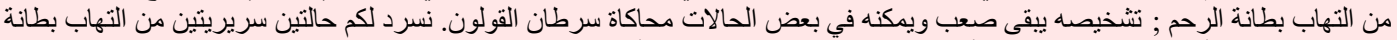

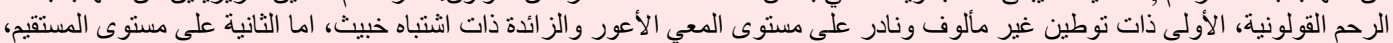

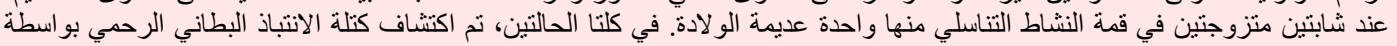

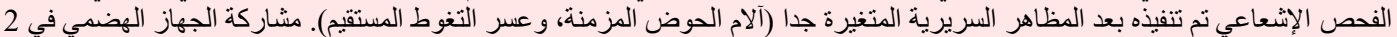

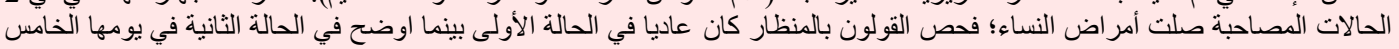

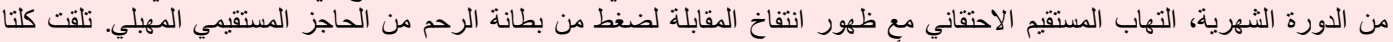

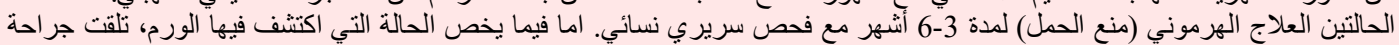
جذرية. كان التطور إيجابيا تحت العلاج في كلتا الحالتين.

كلمات البحث: التهاب بطانة الرحم، التهاب المستقيم، العلاج الجراحي 


\section{INTRODUCTION}

L'endométriose est une affection gynécologique touchant 5 à $10 \%$ des femmes en activité génitale, $15 \%$ des femmes infertiles et $30 \%$ des femmes infertiles souffrant d'algies pelviennes chroniques [1]. Deux localisations peuvent se voir: pelvienne et extra pelvienne [1]. La localisation intestinale est la plus fréquente de l'endométriose extra pelvienne, elle prédomine au niveau du rectum et du sigmoïde dans $70-88 \%$ des cas, suivie de l'iléon, de l'appendice et du cœcum [2]. Son diagnostic reste difficile et peut même simuler, dans certains cas, un cancer du colon. Nous rapportons 2 observations cliniques d'endométriose cœco-appendiculaire et rectale.

\section{OBSERVATION 1}

Une femme âgée de 40 ans, sans antécédents médicochirurgicaux, mariée depuis 7 ans, nullipare, chez qui on a découvert, à la TDM abdomino-pelvienne, réalisée à la suite d'une exploration plus approfondie d'une douleur pelvienne chronique, une masse caeco-appendiculaire de $30 \mathrm{~mm}$ de diamètre associée à des adénopathies profondes mésentériques (figure1). La coloscopie a été réalisée suite aux données scannographiques, et elle est revenue normale. L'exploration peropératoire a mis en évidence une masse cœco-appendiculaire suspecte de malignité (figure2); la patiente a bénéficié d'une hémicolectomie droite avec anastomose iléo-transverse. L'examen anatomo-pathologique de la pièce opératoire a confirmé le diagnostic de l'endométriose sans signes de malignité (figure3).

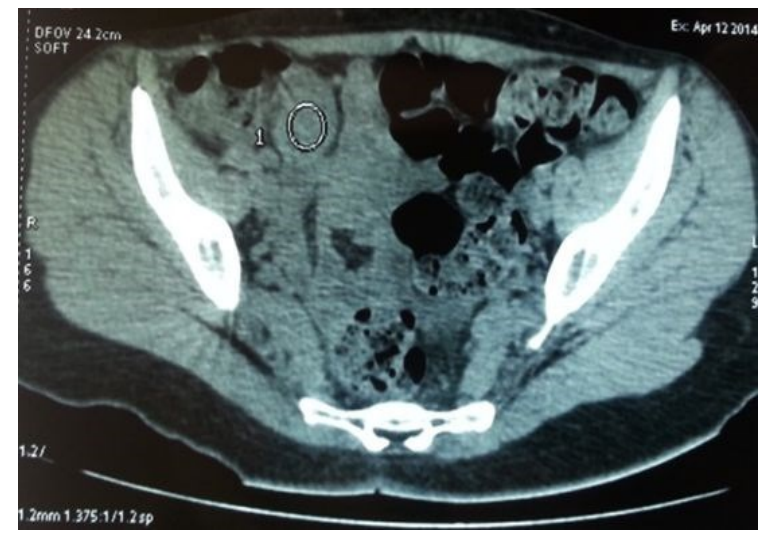

Figure 1: TDM abdomino- pelvienne :masse coeco-appendiculaire + ADP profondes mésentériques.

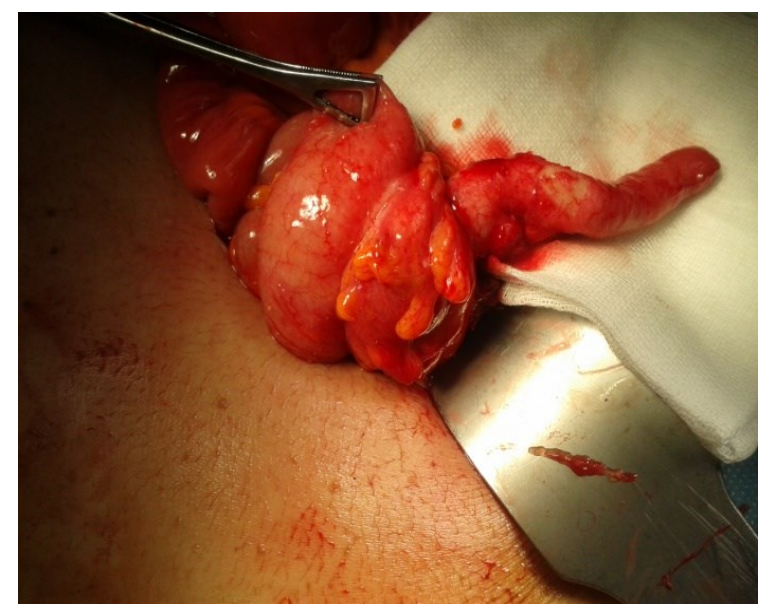

Figure 2 : Laparotomie exploratrice. Masse caeco-appendiculaire.
La patiente a été confiée à une gynécologue pour une recherche de localisation pelvienne de l'endométriose; une échographie endovaginale a été réalisée objectivant de multiples foyers endométriotiques. La patiente est nullipare, elle présente un long passé de douleurs pelviennes chroniques et de dyspareunies profondes. Elle a été mise sous traitement hormonal pendant une durée de 6 mois. L'évolution a été marquée par la disparition totale des douleurs pelviennes juste après la chirurgie, et également la disparation des nodules endométriotiques vaginaux 6 mois après le traitement hormonal.

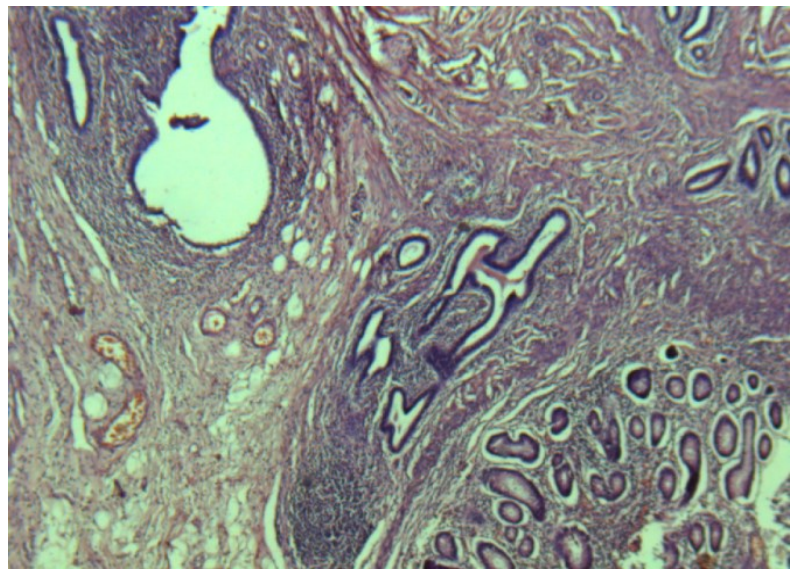

Figure 3: Étude anatomopathologique de la pièce opératoire Endométriose colique + absence de malignité.

\section{OBSERVATION 2}

Une femme âgée de 35 ans mariée et mère de 2 enfants, appendicectomisée à l'âge de 5 ans, s'est présenté pour une dyschésie ano-rectale associée à un syndrome rectal, de survenue de périodique au cours de chaque cycle menstruel depuis plus de 3 ans, exacerbée ses derniers mois de façon permanente, sans émission de sang ou de glaire par l'anus. Par ailleurs, elle ne présente pas de dysménorrhée, de dyspareunie ou de douleur abdominale. Une IRM pelvienne, recommandée par son gynécologue, a été réalisée ; elle met en évidence une adénomyose associée à une endométriose de la cloison recto vaginale antéro-latérale droite mesurant environ $35 \mathrm{~mm}$ de grand axe (figure 4).

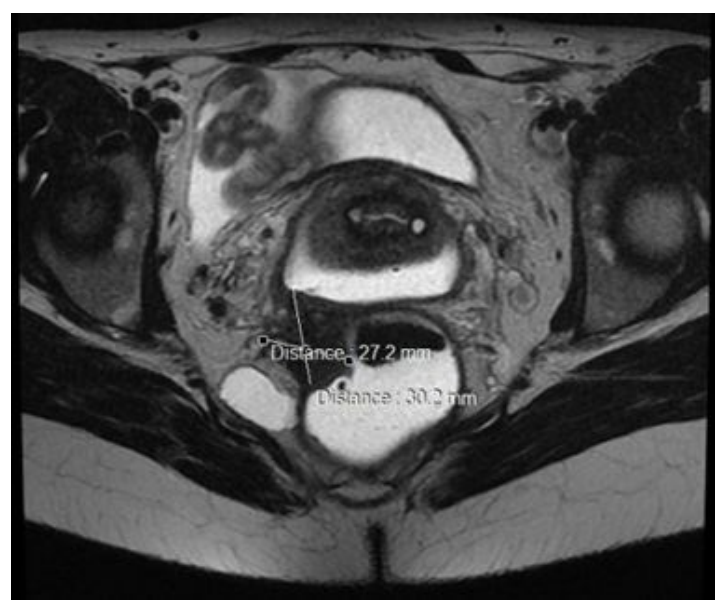

Figure 4 : endométriose de la cloison recto vaginale antéro-latérale droite. 
Au 5 ème jour du cycle menstruel, la patiente a bénéficié d'un dosage du marqueur tumoral CA-125 et d'une rectoscopie. Les résultats retrouvent un taux élevé de CA-125 à 55,69 $\mathrm{UI} / \mathrm{ml}(\mathrm{VN}<35)$ et à la rectoscopie la muqueuse du bas rectum était siège d'une importante congestion avec un aspect bombé de la face antéro-latérale droite (figure 5). Des biopsies ont été réalisées. L'étude anatomopathologique de des biopsies avait mis en évidence une rectite érythémateuse avec une importante suffusion hémorragique inhabituelle (figure 6). La patiente a été mise sous contraception par voie orale pendant une durée de 3 mois. L'évolution a été marquée par la disparition de la dyschésie et du syndrome rectal au cours et après fin de traitement. L'échographie endovaginale de contrôle avait montré une régression importante de la masse endométriale. La chirurgie d'exérèse doit être discutée en réunion de concertation multidisciplinaire en fonction du désir de la grossesse.

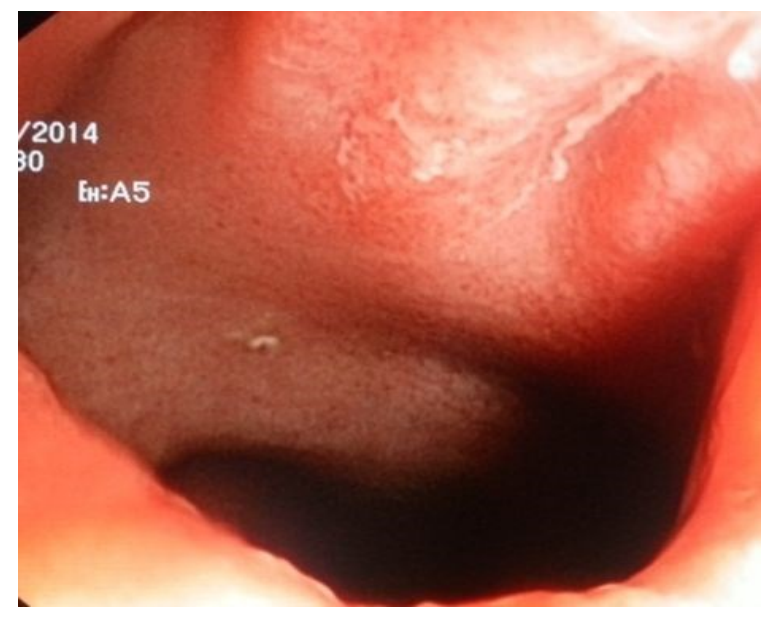

Figure 5 : Rectoscopie (au cours du cycle menstruel) : muqueuse très congestive avec un aspect bombé de la face antéro-latérale droite du bas rectum.

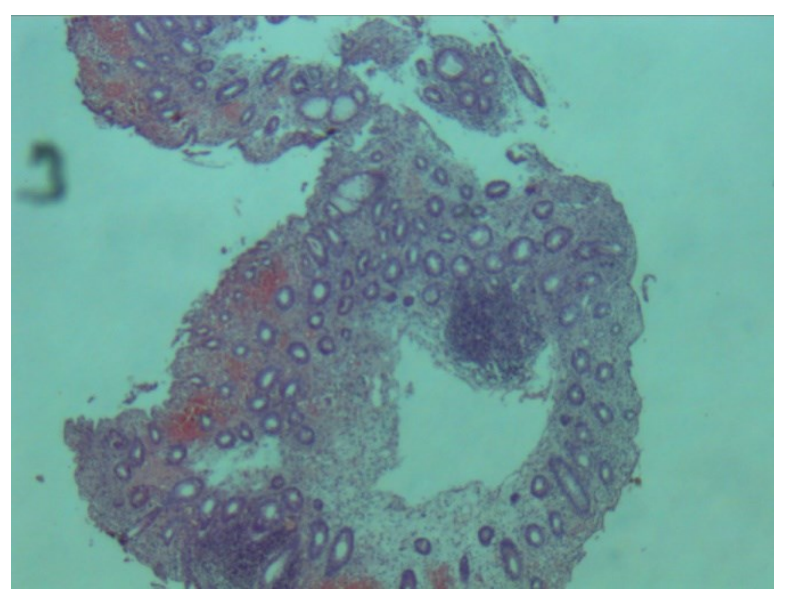

Figure 6 : Étude anatomopathologique de la biopsie rectale: rectite érythémateuse avec une importante suffusion hémorragique.

\section{DISCUSSION}

L'endométriose colique est une forme d'endométriose extra pelvienne profonde qui survient chez des femmes jeunes en activité génitale [1]. La forme caeco-appendiculaire est très rare par rapport à la forme recto sigmoïdienne [3]. Le mode de révélation est variable (symptomatique ou de découverte fortuite), elle peut simuler parfois un cancer du colon [4] comme c'est le cas chez notre première patiente [5]. La forme pelvienne doit être recherchée systématiquement surtout si présence de la triade clinique : infertilité, douleurs pelviennes, dyspareunies.

L'élévation du taux de CA125 au cours de l'endométriose est corrélée à la sévérité de la maladie; sa sensibilité et sa spécificité atteignent respectivement $50 \%$ et $90 \%$ pour l'atteinte endométriosique stade III-IV selon le score AFSR (American Fertility Society Revised) [6,7], qui correspond au stade de sévérité de l'endométriose pelvienne profonde (EPP) postérieure [8].

L'imagerie de l'endométriose est basée sur l'IRM pelvienne, l'écho endovaginale, écho endo rectale. Elle nécessite des opérateurs entrainés $[9,10]$. L'approche thérapeutique est multidisciplinaire, elle est recommandée dans la prise en charge de l'endométriose douloureuse, il est de l'ordre hormonal ou chirurgical $[11,12,13]$.

\section{CONCLUSION}

L'endométriose est une maladie bénigne, chronique, fréquente, qui peut se manifester par des douleurs et/ou une infertilité. La localisation rectale et sigmoïdienne est la plus fréquente de l'endométriose colique contrairement à la localisation appendiculaire et caecale qui est plus rare. La prise en charge de l'endométriose colique est multidisciplinaire; elle fait intervenir le gastroentérologue, le gynécologue et le chirurgien.

Déclaration d'intérêts : les auteurs ne déclarent aucun conflit d'intérêt en rapport avec cet article.

\section{RÉFÉRENCES}

1. Palazzo L, Roseau G. Comment faire le diagnostic d'une endométriose rectosigmoïdienne ? Post'U (2013) 281-29.

2. Musanda $M$, Bounaas $S$. Endométriose: cause inhabituelle d'occlusion intestinale. J Radiol 2000 ; 81 : 538-541.

3. Dimoulios $P$, loannis $E$ koutroubakis. $A$ case of sigmoid endometriosis difficult to differentiate from colon cancer, BMC Gastroenterology 2003, 3:18.

4. Kondo W, Ribeiro R et al. Laparoscopic Treatment of Deep Infiltrating Endometriosis Affecting the Rectosigmoid Colon: Nodulectomy or Segmental Resection? Gynecol Obstetric 2013, S3 :001.

5. Prise en charge de l'endométriose. Recommandations pour la pratique clinique. Texte des recommandations. J Gynécol Obstét Biol Reprod 2007 ; 36 : 186-190.

6. Katsikogiannis N, Tsaroucha AK. Case report Rectal endometriosis causing colonic obstruction and concurrent endometriosis of the appendix: Journal of Medical Case Reports 2011, 5:320.

7. Mol BW, Bayram N, Lijmer JG, et al. The performance of CA125 measurement in the detection of endometriosis: a meta- analysis. Fertil Steril 1998;70 (6):1101-8. 


\section{CAS CLINIQUE}

8. Fritel X. Les formes anatomocliniques de l'endométriose. J Gynécol Obstet Biol Reprod 2007;36:113-8.

9. Nezhat C, King LP. Robotic Assisted Laparoscopic Treatment of Bowel, Bladder and Ureter Endometriosis. JSLS; 2011 JulSep;15(3):387-92.

10. Revised American Fertility Society classification of endometriosis. Fertil Steril 1985;43:351- 2.
11. Senapati S, Barnhart K. Managing endometriosis-associated infertility. Clin Obstet Gynecol. 2011; 54: 720-726.

12. Lockyer C. Adenomyoma in the recto-uterine and recto-vaginal septa. Proc Roy Soc Med 1913;4:112 - 16.

13. Ferrero S, Camerini G. Bowel endometriosis: recent insights and unsolved. World J Gastrointest Surg. Mar 27, 2011; 3(3): 31-38.

Cet article a été publié dans le "Batna Journal of Medical Sciences » BJMS, l'organe officiel de "I'association de la Recherche Pharmaceutique - Batna »

Le contenu de la Revue est ouvert « Open Access » et permet au lecteur de télécharger, d'utiliser le contenu dans un but personnel ou d'enseignement, sans demander l'autorisation de l'éditeur/auteur.

Avantages à publier dans BJMS :

- Open access : une fois publié, votre article est disponible gratuitement au téléchargement

- Soumission gratuite : pas de frais de soumission, contrairement à la plupart des revues « Open Access $\gg$

- Possibilité de publier dans 3 langues : français, anglais, arabe

- Qualité de la relecture : des relecteurs/reviewers indépendants géographiquement, respectant l'anonymat, pour garantir la neutralité et la qualité des manuscrits.

Pour plus d'informations, contacter BatnaJMS@gmail.com

ou connectez-vous sur le site de la revue : www.batnajms.com 


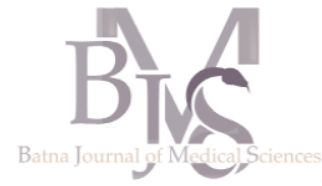

Service de Rhumatologie, Centre hospitalier universitaire Ibn Rochd, Quartier des hôpitaux, Casablanca - Maroc.

\section{Correspondance à :}

Wafa RACHIDI

wafae2403@yahoo.fr

DOI :https://doi.org/10.48087/ BJMScr.2015.2121

\section{Il s'agit d'un article en libre} accès distribué selon les termes de la licence Creative Commons Attribution International License (CC BY 4.0), qui autorise une utilisation, une distribution et une reproduction sans restriction sur tout support ou format, à condition que l'auteur original et la revue soient dûment crédités.

\section{Infarctus musculaire et diabète}

\section{Muscle infarction and diabetes}

\section{Wafae Rachidi, Kawtar Nassar, Saadia Janani, Ouafa Mkinsi}

\section{RÉSUMÉ}

L'infarctus musculaire est une complication rare et souvent méconnue du diabète ancien déséquilibré et multicompliqué. Nous rapportons le cas d'une patiente âgée de 56 ans, diabétique type II mal suivie depuis 14 ans, qui présentait une tuméfaction douloureuse de la cuisse gauche évoluant depuis trois semaines dans un contexte de fièvre et de fléchissement de l'état général. La biologie montrait une glycémie à $3,2 \mathrm{~g} / \mathrm{l}$, une hémoglobine glyquée à $8 \%$, une vitesse de sédimentation à $86 \mathrm{~mm}$ à la première heure et une protéine $\mathrm{C}$ réactive à $160 \mathrm{mg} / \mathrm{l}$. Le diagnostic d'infarctus musculaire, suspecté sur la clinique et les résultats de l'imagerie, a été confirmé par la biopsie musculaire. Cette dernière a été indiquée devant ce tableau atypique, permettant d'écarter les autres causes de tuméfaction musculaire douloureuse en particulier infectieuses. L'évolution clinique était favorable sous insulinothérapie et traitement symptomatique. Mais la patiente a présenté une récidive sur le mollet droit après six mois. Depuis sa première description par Angervall et Stener en 1965, plus de 200 cas d'infarctus musculaire diabétique ont été publiés. Sa physiopathogénie est incertaine. L'hypothèse la plus retenue est l'athérosclérose et la microangiopathie diabétique. La clinique et les données de l'imagerie par résonnance magnétique peuvent suffire au diagnostic. Le traitement est conservateur et l'évolution à court terme est habituellement favorable.

Mots clés: infarctus musculaire, diabète, imagerie par résonance magnétique.

\begin{abstract}
Muscle infarction is a rare and often misdiagnosed complication of long-standing poorly controlled diabetes mellitus. Most patients have associated long term complications. We report a case of a 56-yearold female with 14-year history of type II diabetes who presented painful swelling of her left thigh of three weeks duration, while she had fever and general health decline. Laboratory examinations showed: Blood glucose, $3.2 \mathrm{~g} / \mathrm{l}$; glycated hemoglobin, $8 \%$; erythrocyte sedimentation rate, $86 \mathrm{~mm} / \mathrm{h}$; creactive protein, $160 \mathrm{mg} / \mathrm{l}$. An open muscle biopsy was performed because of the atypical presentation and to rule out other causes of focal pain in patients with diabetes mellitus, especially infections. Patient successfully responded to the received analgesics and aggressive insulin therapy, but she had experienced a recurrence on her right leg after six months. Since it was first described by Angervall et Stener in 1965, diabetic muscle infarction (DMI) has been reported in about 200 cases. The pathogenesis of DMI is unclear. The main hypothesis includes arteriosclerosis and diabetic microangiopathy. Clinical suspicion and characteristic MRI findings are often sufficient to make a diagnosis. Treatment is conservative. Short-term prognosis is good.
\end{abstract}

Keywords: muscle infarction, diabetes mellitus, magnetic resonance imaging.

\section{Pour citer l'article :}

Rachidi W, Nassar K, Janani S, et al. Infarctus musculaire et diabète. Batna J Med Sci 2015;2(1):89-92. https://doi.org/10.48087/ BJMScr.2015.2121

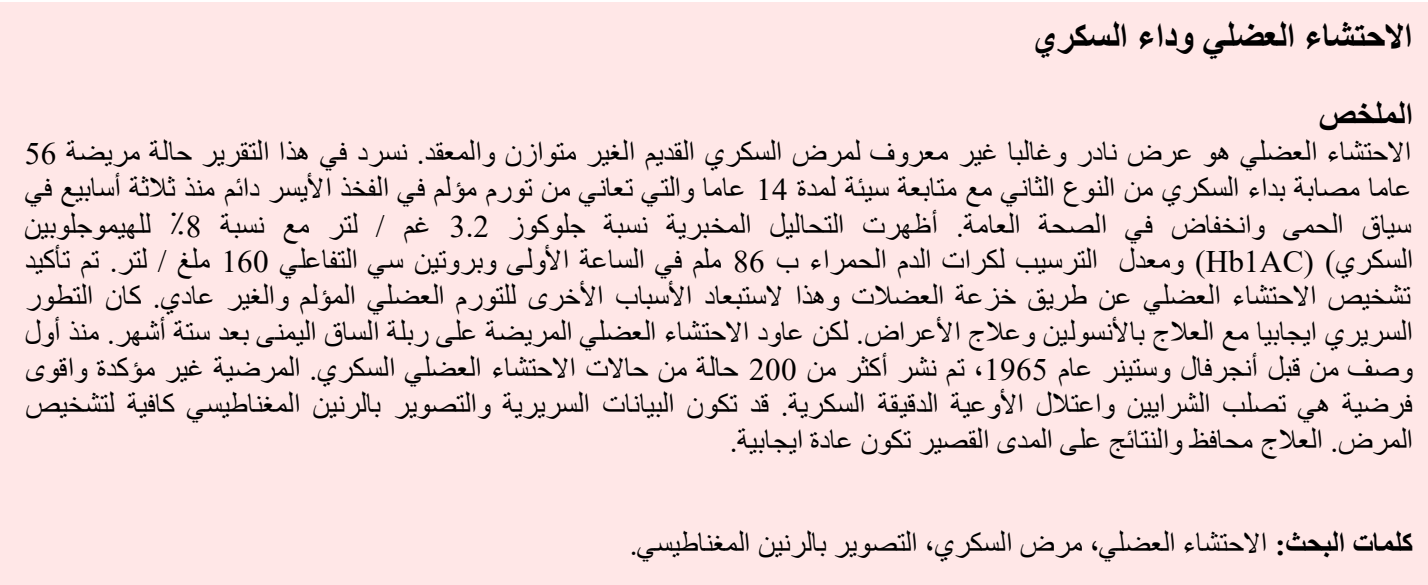




\section{INTRODUCTION}

L'infarctus musculaire diabétique est une entité rare décrite pour la première fois en 1965 par Angervall et Stener qui le nomment " dégénérescence musculaire focale tumoriforme » [1]. Les facteurs de risque associés à cette complication sont : le caractère ancien et insulinodépendant du diabète, le mauvais contrôle glycémique et la présence d'une microangiopathie diabétique $[2,3]$. Nous en rapportons une nouvelle observation.

\section{OBSERVATION}

Une femme de 50 ans était hospitalisée au service de rhumatologie du CHU Ibn Rochd pour une douleur au niveau de la partie interne de l'extrémité inférieure de la cuisse gauche, de caractère inflammatoire évoluant depuis trois semaines, associée à une impotence fonctionnelle du membre inférieur gauche évoluant dans un contexte de fièvre et de fléchissement de l'état général. L'interrogatoire ne retrouvait pas de contexte traumatique, ni de notion d'injection intramusculaire ou d'effort physique intense. La patiente avait pour antécédents un diabète type 2 mal suivi depuis 14 ans, sous insulinothérapie depuis 4 ans, compliqué d'une polyneuropathie et d'une rétinopathie diabétiques et d'une hypertension artérielle. L'examen a retrouvé une fièvre à $40^{\circ} \mathrm{C}$, une augmentation du volume de la cuisse gauche, avec un pourtour quadricipital gauche à plus $8 \mathrm{~cm}$ par rapport au droit, un point douloureux à la face interne de la cuisse gauche et une arthrite du genou gauche. Les examens biologiques ont révélé un syndrome inflammatoire avec une vitesse de sédimentation à $86 \mathrm{~mm}$ à la première heure et une protéine c réactive à $160 \mathrm{mg} / \mathrm{l}$. L'hémogramme était correct. Les hémocultures étaient négatives. Les enzymes musculaires étaient normales. Il y'avait une hyperglycémie à jeun à 3,2 g/l avec une hémoglobine glyquée à $8 \%$. La ponction articulaire du genou gauche a révélé un liquide articulaire mécanique stérile $\left(\mathrm{GB}<15\right.$ éléments $\left./ \mathrm{mm}^{3}\right)$. La radiographie standard du genou a été normale. L'échodoppler veineux des membres inférieurs n'a pas montré de thrombose veineuse profonde. L'échographie des parties molles a montré une plage hypoéchogène au niveau des muscles de la face interne de la cuisse gauche sans collection individualisable, une infiltration œdémateuse très importante du tissu cellulaire sous cutané avec une involution graisseuse des muscles.

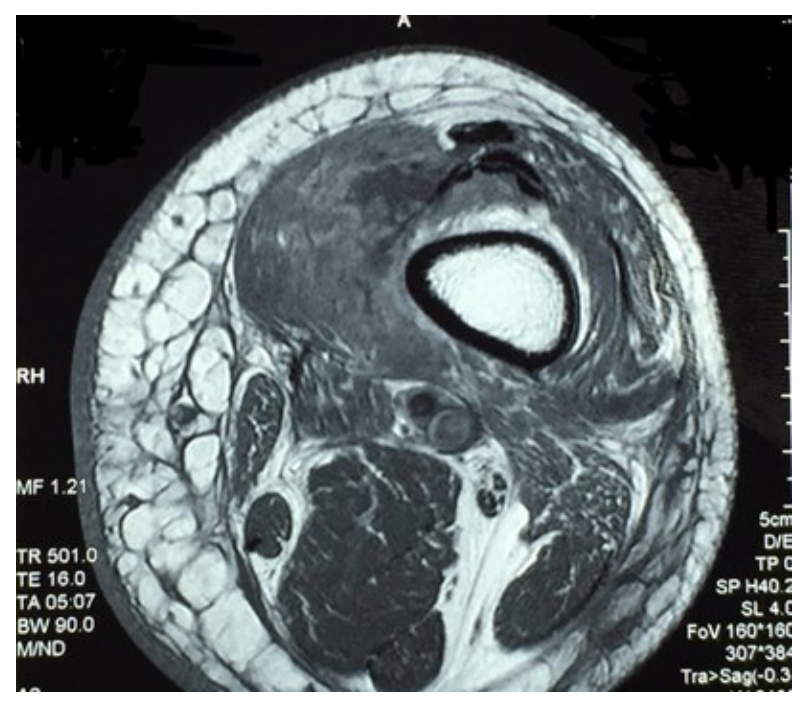

Figure 1 : Coupe axiale de la cuisse gauche : processus en hyposignal $\mathrm{T} 1 \mathrm{au}$ niveau du vaste interne gauche.
L'IRM de la cuisse gauche a montré un processus mal limité du vaste interne hypointense en $\mathrm{T} 1$, hyperintense en $\mathrm{T} 2$, avec une prise de contraste hétérogène significative à l'injection de gadolinium (Figures 1 et 2). En per-opératoire, le muscle est apparu oedématié, infiltré par un tissu blanchâtre. La biopsie musculaire a pu écarter les diagnostics différentiels, en montrant des zones de nécrose des fibres musculaires et un infiltrat de polynucléaires neutrophiles et de monocytes mononucléés. L'évolution était favorable après trois semaines sous traitement symptomatique associant un antalgique, un anti-inflammatoire non stéroïdien et le repos.

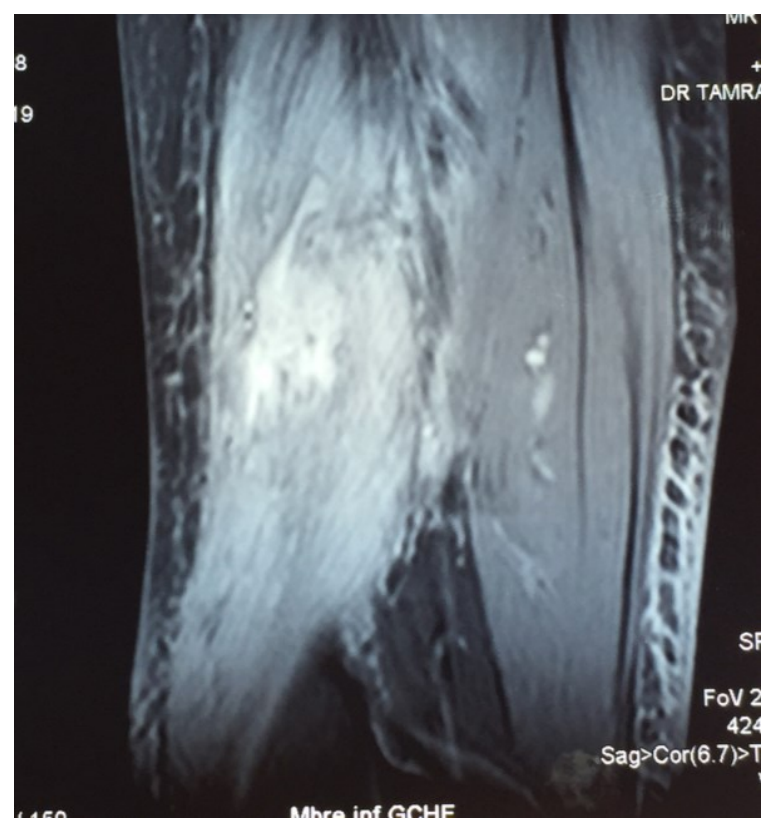

Figure 2: IRM en coupe sagittale en pondération T2 STIR, lésion du muscle vaste interne gauche, en hypersignal,hétérogène de contours mal limités .

Après six mois, la patiente a présenté une récidive sur le mollet droit. Le bilan réalisé a trouvé un diabète toujours déséquilibré avec une glycémie à $1,74 \mathrm{~g} / \mathrm{l}$ et $\mathrm{Hb}$ glyquée à $11,4 \%$. La patiente fut ré-adressée en consultation d'endocrinologie pour une bonne prise en charge de son diabète.

\section{DISCUSSION}

L'infarctus musculaire est une complication rare et souvent méconnue du diabète ancien déséquilibré. Il est plus fréquemment rencontré chez les femmes (61\%) avec un âge moyen de 42,6 ans (extrêmes : 13- 81 ans) [2]. Cependant, ce sexe ratio tend à s'inverser chez les diabétiques hémodialysés [4].

Sa physiopathologie demeure incertaine. Plusieurs hypothèses ont été évoquées: la variabilité glycémique, l'athérosclérose et les troubles de la coagulation à type d'hypercoagulabilité avec augmentation des concentrations du facteur VII, fibrinogène et thrombomoduline ; la présence d'anticorps anti-phospholipides et une diminution des taux de prostacycline, antithrombine et de l'activateur tissulaire du plasminogène. La plus retenue étant la microangiopathie diabétique. En effet, cette complication survient chez des patients ayant un diabète ancien évoluant depuis près de 14 ans en moyenne, préférentiellement de type 1 au stade de complications microvasculaires. Chez ces patients; la 
néphropathie, la rétinopathie et la neuropathie sont présentes dans $71 \%, 57 \%$ et $55 \%$ des cas respectivement $[2,5,6]$.

L'IMD se présente typiquement par une tuméfaction musculaire très douloureuse de début brutal du membre inférieur (cuisse ou mollet) sans facteur déclenchant en particulier traumatique. L'atteinte du membre supérieur a été décrite, mais reste exceptionnelle [4,7]. Deux cas d'atteinte de la paroi abdominale antérieure et du grand pectoral ont également été rapportés [8]. Les formes bilatérales représentent $8 \%$ des cas. Une impotence fonctionnelle peut être associée. La fièvre est présente dans $10 \%$ des cas [2].

La biologie est peu contributive au diagnostic. Un syndrome inflammatoire modéré est présent dans $53 \%$ des cas. L'hyperleucocytose peut être modérée $(8 \%)$ ou absente. Les enzymes musculaires sont normales ou modérément élevées (dans $50 \%$ des cas) en particulier au début. L'absence de corrélation entre l'élévation de la créatine kinase et l'atteinte musculaire peut être expliquée par un retard de consultation [2]. Ceci est corroboré par notre patiente qui a consulté 3 semaines après le début des symptômes, et chez qui le dosage des CPK et aldolases était normal. L'imagerie par résonnance magnétique est l'examen de choix pour le diagnostic de l'infarctus musculaire diabétique en raison de sa haute sensibilité et de son caractère non invasif. L'aspect typique est une augmentation du volume de l'aire musculaire (intraet périmusculaire) qui apparait en iso- ou hyposignal $\mathrm{T} 1$, hypersignal diffus en $\mathrm{T} 2$ et prenant le contraste après injection de gadolinium. Ceci est en rapport avec les phénomènes inflammatoires et oedémateux accompagnant l'infarctus musculaire. Dans $90 \%$ des cas, on retrouve un œdème sous-cutané et un épaississement des septas musculaires $[9,10]$.

La biopsie musculaire est le gold standard pour le diagnostic. Elle permet de révéler des foyers de nécrose musculaire, un infiltrat de cellules inflammatoires et des anomalies microvasculaires, comme ce qui a été noté chez notre malade. Cependant, elle ne doit pas être systématique en raison du risque d'exacerbation aigue, de surinfection et de retard de guérison évoqué par certains auteurs. De ce fait, elle devra être réservée aux patients ayant une présentation atypique ou en l'absence d'amélioration sous traitement symptomatique $[7,11]$.

Notre patiente était fébrile. Son bilan ne retrouvait pas d'hyperleucocytose, mais le syndrome inflammatoire était majeur avec une CRP très élevée à $160 \mathrm{mg} / \mathrm{l}$, ce qui a motivé la réalisation de la biopsie musculaire afin d'éliminer essentiellement une cause infectieuse ou inflammatoire.

Les diagnostics différentiels de l'infarctus musculaire sont présentés dans le tableau 1. Les infections doivent être évoquées en premier (pyomyosite, ostéomyélite, abcès, cellulite et fasciite nécrosante) d'autant plus qu'elles sont fréquentes sur un terrain diabétique. En faveur de ces étiologies, on retiendra la fièvre, l'hyperleucocytose, la présence à l'imagerie d'un renforcement des fascias, ou d'anneau entourant des collections individualisées [12]. Il convient d'éliminer en priorité la fasciite nécrosante, infection fulminante mettant en jeu le pronostic vital, et qui nécessite une prise en charge chirurgicale urgente si elle est suspectée [13]. Le caractère aigu et bruyant de la symptomatologie douloureuse et l'absence de masse compressive permettent de le différencier d'un processus tumoral. La normalité du taux des D-dimères et de l'échodoppler veineux des membres permettent d'écarter une thrombose veineuse profonde.
Tableau 1: Diagnostics différentiels de l'infarctus musculaire diabétique

Infections Pyomyosite, abcès, cellulite, fasciite nécrosante, ostéomyélite

Traumatisme Hématome, rupture musculaire, myosite ossifiante

Tumeurs Bénignes: lipome, chondrome, fibrome, leiomyome

Malignes: lymphome musculaire ou osseux, rhabdomyosarcome, liposarcome, fibrosarcome

Pathologies vasculaires

Thrombose veineuse profonde, syndrome compartimental aigu, lymphœdème, hémangiome

Pathologies Myosite focale, fasciite proliférante, musculaires inflammatoires

Autres polymyosite, dermatomyosite

Rupture de kyste poplité, myopathie médicamenteuse, amyotrophie diabétique, amylose

Il n’y a pas de traitement spécifique. Il est recommandé de proposer le repos et des traitements antalgiques avec recours prudent aux anti-inflammatoires non stéroïdiens si nécessaire (insuffisance rénale fréquente sur ce terrain) [2, 11]. Un contrôle optimal du diabète et l'arrêt du tabagisme sont vivement conseillés. Dans une analyse de cas d'infarctus musculaire comparant le devenir des patients sous traitement médical ou chirurgical. Il ressort que le temps de guérison était significativement plus long chez les patients ayant subi une excision chirurgicale du muscle nécrosé que ceux ayant eu un traitement conservateur (13 semaines versus 5,5) [3]. Certains auteurs proposent un traitement par antiagrégants plaquettaires tels l'aspirine à $80 \mathrm{mg} / \mathrm{j}$ (faible niveau de preuve) en raison de leur efficacité supposée sur la microangiopathie sous-jacente afin de prévenir les récidives [14]. D'autres thérapeutiques pourraient être bénéfiques: pentoxiphylline, dipyridamole et la nifedipine. Néanmoins, il n'y a aucun essai randomisé validant ces agents dans cette indication [13]. La rééducation physique et la pratique d'activités non en charge après la phase aigue peuvent être bénéfiques [10]. Par contre, une rééducation intensive ou une reprise trop précoce de la marche peuvent être sources d'exacerbation douloureuse [14].

Le pronostic à court terme est habituellement bon avec amélioration spontanée en quelques semaines. Cependant, les complications microvasculaires du diabète, généralement associées, sont à l'origine d'un mauvais pronostic à long terme avec une surmortalité dans les deux ans qui suivent. Des récidives peuvent survenir dans un délai variable dans $50 \%$ des cas. Près de la moitié de ces récurrences surviennent après un intervalle de 2 mois par rapport à l'épisode initial, de siège homolatéral ou controlatéral $[2,11,13]$. Ce fut le cas chez notre malade.

\section{CONCLUSION}

La myonécrose diabétique est une complication encore méconnue et sous diagnostiquée. Nous assisterons probablement à une augmentation de son incidence en raison de la prévalence croissante du diabète dans notre société. De ce fait, il est nécessaire de l'évoquer devant toute douleur musculaire brutale chez un patient diabétique, à fortiori si diabète de type 1 ancien et déséquilibré au stade de complications microvasculaires. La biopsie musculaire n'est 
pas indispensable. Le traitement est essentiellement symptomatique.

Déclaration d'intérêts : les auteurs ne déclarent aucun conflit d'intérêt en rapport avec cet article.

\section{RÉFÉRENCES}

1. Angervall L, Stener B. Tumoriform focal muscular degeneration in two diabetic patients. Diabetologia $1965 ; 1$ : 39-42.

2. Trujillo-Santos AJ. Diabetes muscle infarction. An underdiagnosed complication of long-standing diabetes. Diabetes Care 2003; 26:211-5.

3. Kapur S, McKendry RJ. Treatment and outcomes of diabetic muscle infarction. J Clin Rheumatol. 2005 Feb;11(1):8-12.

4. Joshi R, Reen B, Sheehan H. Upper extremity diabetic muscle infarction in three patients with end-stage renal disease: a case series and review. J Clin Rheumatol. 2009 Mar;15(2):81-4.

5. Meas T, Virally M, Kevorkian JP, et al. Infarctus musculaire chez une femme ayant un diabète de type 1 instable. Rev Med Int 2007;28:S123-4.

\section{RÉFÉRENCES}

6. Virally $\mathrm{M}$, Laloi-Michelin $\mathrm{M}$, Médeau $\mathrm{V}$, and al. Muscle infarction in a young woman with brittle type 1 diabetes. Diabetes \& Metabolism 2007 ; 33 : 466-468.

7. Mukhopadhyay P, Barai R, Philips C A, et al. An Unusual Case of Myonecrosis. Case Rep Endocrinol 2011; Vol 2011: 624020, 4 pages.

8. Ran X, Wang C, Wang H, Zhao T, Tong N, Song B, et al. Muscle infarction involving muscles of abdominal and throracic walls in diabetes. Diabet Med 2005;22:1757-60.

9. Kattapuram TM, Suri R, Rosol MS, and al. Idiopathic and diabetic skeletal muscle necrosis: evaluation by magnetic resonance imaging. Skeletal Radiol 2005 Apr;34(4):203-9.

10. Sran S, Sran M, Ferguson N, et al. Diabetic Myonecrosis: Uncommon Complications in Common Diseases. Case Rep Endocrinol 2014 ; Vol 2014 : 175029, 3 pages.

11. Bouchaud-Chabot A. Infarctus musculaire, le diabétique et les autres. Rev Rhum 2008 ; 75 : 166-168.

12. Mazoch MJ, Bajaj G, Nicholas R, et al. Diabetic Myonecrosis: Likely an Underrecognized Entity. Orthopedics 2014 Oct ; 37(10) : 936- 9.

13. Iyer SN, Drake AJ third, West RL, et al. Diabetic Muscle Infarction: A Rare Complication of Long-Standing and Poorly Controlled Diabetes Mellitus. Case Rep Med 2011; Vol 2011: 407921, 4 pages.

14. Litvinov IV, Radu A and Garfield N. Diabetic muscle infarction in a 57 year old male: a case report. BMC Research Notes 2012 ; 5: 701.

Cet article a été publié dans le « Batna Journal of Medical Sciences » BJMS, l'organe officiel de "I'association de la Recherche Pharmaceutique - Batna »

Le contenu de la Revue est ouvert "Open Access » et permet au lecteur de télécharger, d'utiliser le contenu dans un but personnel ou d'enseignement, sans demander l'autorisation de l'éditeur/auteur.

Avantages à publier dans BJMS :

- Open access : une fois publié, votre article est disponible gratuitement au téléchargement

- Soumission gratuite : pas de frais de soumission, contrairement à la plupart des revues « Open Access 》

- Possibilité de publier dans 3 langues : français, anglais, arabe

- Qualité de la relecture : des relecteurs/reviewers indépendants géographiquement, respectant l'anonymat, pour garantir la neutralité et la qualité des manuscrits.

Pour plus d'informations, contacter BatnaJMS@gmail.com

ou connectez-vous sur le site de la revue : www.batnajms.com 


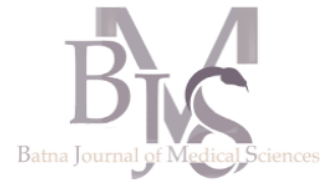

Service de Radiologie - CHU Batna, Algérie

\section{Correspondance à :}

Dr. Athmane SALAH SALAH

atman 311@hotmail.com

DOI :https://doi.org/10.48087/ B]MSicm.2015.2122

Il s'agit d'un article en libre accès distribué selon les termes de la licence Creative Commons Attribution International License (CC BY 4.0), qui autorise une utilisation, une distribution et une reproduction sans restriction sur tout support ou format, à condition que l'auteur original et la revue soient dûment crédités.

Pour citer l'article :

Salah Salah A. Spondyloarthrite diagnostiquée lors d'un bilan d'une maladie de Crohn compliquée. Batna J Med Sci 2015;2(1):93.

https://doi.org/10.48087/BJMS icm.2015.2122

\section{Spondyloarthrite diagnostiquée lors du bilan d'une maladie de Crohn compliquée}

\section{Spondyloarthritis diagnosed when exploring a complicated Crohn's disease}

\section{Athmane Salah Salah}
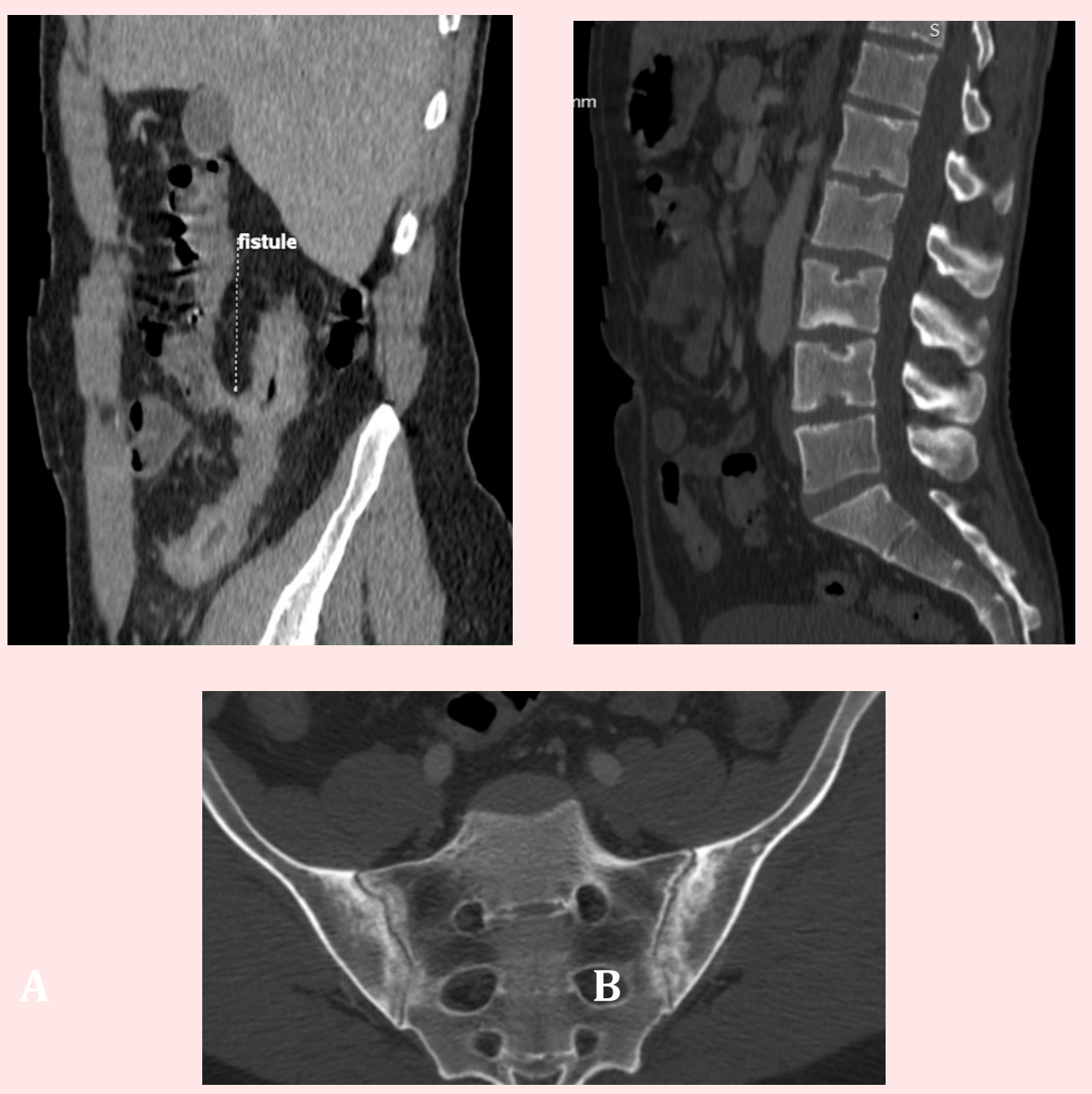

Patient âgé de 30 ans, atteint d'une maladie de Crohn diagnostiquée histologiquement, adressé pour une tomodensitométrie (TDM) abdominopelvienne, à la recherche de complications type fistule. Le diagnostic d'une maladie de Crohn fistulisée a été confirmé par la TDM (Figure A). Cependant, malgré l'indolence du patient, des lésions typiques de spoiidyloarthrite $(\mathrm{SpA})$ ont été détectées lors de cet examen tomodensitométrique, à type de spondylodiscite multiétagée prédominant en L2-L3, L3-L4 et L4-L5 (Figure B), ainsi qu'une sacroiliite bialtérale grade III (Figure C). Devant le contexte (jeune homme, maladie de Crohn active) et l'aspect scannographique typique, le diagnostic de sponyloarthrite est certain. Reste à connaître les répercussions de ce diagnostic sur le plan thérapeutique.

\section{Association ?}

Environ 20\% des patients atteints d'une maladie de Crohn développent une SpA, et vice versa. L'évolution de la SpA axiale (rachis et sacro-iliaques) est indépendante de l'évolution de la maladie de Crohn. La prise en charge thérapeutique pose un véritable défi, puisque le traitement de référence des $\mathrm{SpA}$, les antiinflammatoires non stéroïdiens (AINS) sont souvent contre-indiqués chez les patients atteints de maladie de Crohn. Le recours aux anti-TNF alpha, comme alternative thérapeutique, est fréquent. 


\title{
$1^{1 \text { ère }}$ conférence internationale de Guelma sur les hépatites $(\mathrm{CIGH})$
}

\author{
Est-ce que l'ère d'éradication des hépatites virales a débuté ?
}

Université de Guelma, 9 avril 2015

\section{ABSTRACTS DES CONFÉRENCES}

Les cibles thérapeutiques des hépatites virales $B$ et $C$

K Amoura, A Dahi, S Amiri, A Otmane, S Nedjai, ML Meghadecha, A

Mammeri, M Dekhil

Faculté de médecine Annaba

Avec 2 milliards d'individus infectés dans le monde par le virus de l'hépatite B et 170 millions infectés par le virus de l'hépatite $C$; les hépatites virales $B$ et $C$ constituent un problème de santé publique mondial. L'enjeu du traitement de ces hépatites virales chroniques est d'inhiber la réplication virale afin d'éviter l'évolution vers la cirrhose et le carcinome hépatocellulaire. Le traitement des hépatites virales chroniques a considérablement progressé ces dernières années avec l'apparition de nouvelles molécules antivirales. Les thérapies classiques avec l'interféron ont évolué vers les bi et trithérapies avec l'utilisation des analogues nucléosidiques pour l'hépatite B et les inhibiteurs de la protéase pour le virus de l'hépatite C. Cependant et malgré la disponibilité des nouvelles molécules le traitement des hépatites virales reste un véritable défi. L'enjeu de la recherche actuelle est de développer de nouvelles molécules ciblant directement les virus. L'amélioration des stratégies antivirales repose sur l'utilisation de combinaisons ciblant différentes étapes du cycle de multiplication pour obtenir un meilleur taux de réponse virologique. Une connaissance approfondie des interactions entre le virus et son hôte est indispensable pour comprendre l'évolution de la maladie et développer de nouvelles approches thérapeutiques.

\section{Traitement futur de l'hépatite $B$ chronique : une mini revue M Laouar \\ Université Badji Mokhtar, CHU Annaba}

Contexte: À la fin de cette année 2014 l'actualité concernant l'hépatite B chronique est marquée sur le plan épidémiologique par la persistance d'un nombre très important dans le monde de patients atteints par cette maladie avec toujours la même morbi-mortalité très importante faisant toujours de l'hépatite $\mathrm{B}$ chronique la première cause de transplantation hépatique dans le monde. En opposition aux avancées spectaculaires enregistrées dans le traitement de l'hépatite $C$, surtout avec l'avènement des antiviraux d'action directe où le but actuel du traitement est devenu non seulement la guérison de la maladie mais aussi l'éradication du virus C, le traitement de l'hépatite chronique $B$ est en stagnation et les molécules disponibles actuellement ne permettent pas d'atteindre le gold standard du traitement qui est la séroconversion de l'AgHBs. Dans cette revue de la littérature, on sélectionnera et détaillera les résultats des pistes de recherche les plus intéressantes sur les nouvelles molécules qui seront destinées au traitement de l'hépatite $B$ chronique. Méthodes : Une recherche initiale a été conduite dans les bases de données PubMed et Google scholar en utilisant les termes suivants: "therapeutic advances in hepatitis $b$; new therapeutic advances in hepatitis $b, H B V$ review, advances in hepatitis $b$ ». Tous les résultats rapportant des nouvelles stratégies ou de nouvelles molécules thérapeutiques utilisées dans le traitement de l'hépatite B chronique ont été retenus. Par la suite, les abstracts été lus avec accès au document concerné en full text par le biais de la plateforme SNDL donnant un accès aux documents en texte intégral via certaines bases de données telle que sciencedirect et MEDLINE Complete. Résultats : Plusieurs nouvelles molécules anti-VHB en développement dont les mécanismes et sites d'actions sont différents, sont prometteuses : les nouvelles molécules d'analogues, à l'exemple du Besifovir, Tenofovir Alafenamide et le CMX157, les inhibiteurs d'entrée du VHB, surtout le Myrcludex B, d'autres substances telles que la Cyclosporine A et Ezetimibe, les inhibiteurs d'assemblage de la capside comme les
HAP, les Phenylpropenamides et les Sulfamoylbenzamides, les inhibiteurs de libération de l'AgHBs (le REP 9 AC), les immunomodulatuers (agoniste des toll like receptor 7, et activateurs du récepteur lymphotoxin- $\beta$, les inactivateurs des gènes (ARC-520) et la Vaccinothérapie dans un cadre curatif et non préventif (GS-4774). Conclusion : Les nouvelles avancées thérapeutiques contre l'hépatite B restent à l'heure actuelle au stade des essais thérapeutiques. Certaines pistes sont encourageantes et ouvrent des perspectives qui peuvent aboutir même à une vraie clairance de l'AgHBs et dont le but est d'atteindre le gold standard du traitement à savoir la séroconversion $\mathrm{S}$.

\section{Réactivation virale B \\ M Messast \\ Clinique d'Infectiologie. CHU Benbadis, Constantine}

$\mathrm{Au}$ cours d'une chimiothérapie par immunosuppresseurs, une réactivation peut survenir, non seulement, chez les porteurs inactifs d'Ag HBs mais aussi chez les patients guéris d'une hépatite virale B (Ag HBs négatif, Ac $\mathrm{HBc}$ positif/Ac HBc positif). La réactivation plus grave qu'une hépatite aiguë (mortalité de $26 \%$ vs $4 \%$ ). Une réactivation est définie, chez les porteurs d'Ag HBs par une réversion de l'Ag HBe qui redevient positif et l'élévation de la charge virale et chez le patients Ag HBs négatifs, Ac HBc positifs/Ac HBs positifs par la réapparition de l'Ag $\mathrm{HBs}$ et une charge virale qui redevient détectable. Le risque de réactivation est plus élevé quand le traitement immunosuppresseur contient du Rituximab et/ou des stéroides. Le traitement pré-emptif est impératif avant de débuter une chimiothérapie chez les patients porteurs d'Ag HBs par de la Lamuvidine ou mieux par de l'Entécavir ou du Ténofovir. Chez les patients Ag HBs négatifs, Ac anti-HBc positifs la charge virale est surveillée périodiquement et le traitement est institué par de l'Entécavir ou du ténofovir quand la CV se positive. Conclusion : La réactivation virale $B$ peut survenir chez tout patient ayant été en contact avec le virus $B$ et recevant un traitement immunosuppresseur. La prévention de cette réactivation nécessite une sérologie systématique de l'hépatite virale $B$ et un traitement pré-emptif.

Efficacité de l'Entecavir chez les patients avec hépatite $B$ chronique : Notre expérience après cinq ans de traitement ML Meghadecha ${ }^{1}$, S Boulanouar ${ }^{2}$, A Mammeri $^{1}, Z$ Boumaza $^{1}, M$ Aidaoui $^{1}$, K Amoura ${ }^{3}$, M Laouar ${ }^{1}$

${ }^{1}$ Université Badji Mokhtar, Service d'infectiologie, CHU Annaba, Annaba, Algérie ${ }^{2}$ EPH Ibn Zohr ,Guelma, Algérie. ${ }^{3}$ Université Badji Mokhtar, Service de microbiologie, CHU Annaba, Annaba, Algérie

Contexte : La suppression de la réplication virale de façon continue améliore de façon très considérable la morbi-mortalité résultante de l'hépatite $B$ chronique et l'utilisation d'analogues antiviraux avec une forte action antivirale et une haute barrière de résistance génétique $y$ contribue efficacement. But: Déterminer la réponse virobiochimique de l'Entécavir dans le traitement de l'Hépatite B chronique après cinq ans de traitement. Méthode: Etude rétrospective et prospective, période rapportée du 01/01/2010 au 28/02/2015 d'un total de 40 patients atteints d'Hépatite B chronique, traités par Entécavir. 32 patients naïfs d'Analogues sous Entécavir 0,5 mg/j, Entécavir $1 \mathrm{mg} / \mathrm{j}$, Entécavir $0,5 \mathrm{mg} / \mathrm{semaine}$ (respectivement pour 27, 04, 01 patients) et 08 patients expérimentés aux analogues sous Entécavir $1 \mathrm{mg} / \mathrm{j}$ (ayant reçu pendant une période limitée de 48 semaines de la Lamivudine). Résultats : l'âge moyen des patients était de 48 ans \pm 12 ans , $72 \%$ de sexe masculin ,89\% AgHBe négatif, $86 \%$ avaient une 
charge virale faible $<7 \log \mathrm{UI} / \mathrm{ml}, 86 \%$ des patients avait une fibrose modérée à sévère et $28 \%$ avaient une cirrhose dont quatre avec une cirrhose décompensée, $67 \%$ des patients avait des ALAT > LSN avant le début du traitement. Sous Entecavir on n'a pas constaté de non réponse primaire. La réponse virologique et biochimique était respectivement à $94 \%$ et $97 \%(n=36), 93 \%$ et $96 \%(n=27), 100 \%$ et $95 \%(n=22), 100 \%$ et $95 \%(n=19), 100 \%$ et $100 \%(N=11)$, après respectivement un, deux, trois, quatre, cinq ans de traitement. Deux patients ont perdu l'Ag HBs. Conclusion : Le traitement de l'hépatite chronique B par l'Entécavir donne une négativation rapide de la charge virale avec un pourcentage de réponse virologique et biochimique qui augmente avec le temps, atteignant $100 \%$ dans notre étude après cinq ans de traitement.

\section{Coïnfection VIH/VHB : aspects épidémiologiques et traitement}

A Lacheheb, A Gasmi et W Guenifi

Université Ferhat Abbas Sétif-, Faculté de Médecine de Sétif, Centre Hospitalier universitaire de Sétif.

La coïnfection VIH/VHB est une situation fréquente en raison de leurs modes de transmission identiques et de la juxtaposition des zones géographiques de fortes prévalences. Chez les patients vivants avec le VIH (PVIH), les Ac anti HBc sont présents dans 70 à 90\% des cas et l'Ag HBs chez 5 à $20 \%$ des patients. La présence du VHB ne semble pas affecter l'évolution naturelle de l'infection par le VIH de même que la réponse au traitement anti rétroviral. Cependant, l'infection VIH modifie l'histoire naturelle et aggrave le pronostic de l'infection par le VHB. En effet, en cas d'hépatite aiguë, le risque de passage à la chronicité est de 20 à $25 \%$ chez le coïnfecté contre $5 \%$ chez le mono-infectés ; dans les formes chroniques d'HVB, la vitesse de progression de la fibrose est 3 à 6 fois plus importante et le risque d'apparition du carcinome hépato cellulaire (CHC) est majoré. Ainsi, il est actuellement recommandé dans la prise en charge des patients coinfectés VIH/VHB d'instaurer dès que possible les traitements anti viraux, indépendamment du taux des lymphocytes TCD4 et des facteurs habituellement pris en compte dans l'indication du traitement du VHB chez les patients mono-infectés (le taux d'ALAT, le taux ADN viral et le niveau des lésions nécrotico-inflammatoires). Le traitement doit impérativement inclure des molécules actives sur les deux virus ; le ténofovir est un agent privilégié et doit être prescrit si possible en association avec la lamivudine ou l'entricitabine. L'évolution de l'infection par le VHB sous traitement est en général favorable. Les études ont démontré une diminution nette de morbimortalité par rapport aux patients non traités et une chute de la prévalence des cirrhoses décompensées chez les patients traités par multi thérapie incluant le ténofovir.

\section{Traitement actuel de l'hépatite $C$ en Algérie}

N Debzi

Service d'hépatologie CHU Mustapha, Alger.

Sur le plan épidémiologique, l’Algérie est considérée comme une zone géographique de moyenne endémicité, chez les donneurs de sang, la prévalence des anticorps anti-VHC positifs ne dépasse pas $0,40 \%$ (2010, source ANS sur 300.000 donneurs de sang). La population des hémodialysés est la plus exposée, la prévalence de l'infection est de 23,8\% (2008, source MSPRH-IPA). Le génotype 1 est le plus fréquent en Algérie et c'est le plus difficile à traiter (Source Dr S Berkane cohorte nationale 2012, G1 72\%). Sur le plan géographique le G1 est prédominant à l'est (90\%) et au centre (70\%), à l'ouest on retrouve dans des proportions égales le G1 (50\%) et le G2 (50\%). Dans notre pays, la réponse virologique soutenue à la bithérapie Interféron pegylé-Ribavirine est de $59 \%$ pour le G1 (prédominance du sous-type 1b) et de $80 \%$ pour les G 2 et G 3 (source Dr N Debzi cohorte nationale 2012). Une nouvelle ère thérapeutique s'ouvre avec les nouvelles molécules approuvées récemment aux USA et en Europe, comme le sofosbuvir, la combinaison sofosbuvir-ledipasvir et l'association de trois agents antiviraux directs ABT $450 \mathrm{r}$, ABT 267 et ABT 333 , permettant de se passer de l'interféron. Les taux de RVS dépassent 95\%. En Algérie les indications suivantes des «interféron free » peuvent êtres retenues : patients naïs F3 F4, patients non éligibles à la Bithérapie $\mathrm{F} 2 \mathrm{~F} 3 \mathrm{~F} 4$, échecs $\mathrm{Bi}$ et trithérapie de première génération $\mathrm{F} 2 \mathrm{~F} 3 \mathrm{~F} 4$, Co-infectés VHC-VIH F2 F3 F4, manifestations extra-hépatiques. L'introduction large des IFN free en Algérie sera probablement limitée par le coût.
La trithérapie anti-VHC : Expérience du service des Maladies Infectieuses CHU Annaba

A Mammeri, A Touaref, M Meghadecha, Z Boumaza, M Laouar

Service des maladies infectieuses CHU Annaba.

Dans le monde, Jusqu'en 2011, le traitement de l'hépatite C chronique reposait sur une bithérapie associant IFN $\alpha$-PEG et ribavirine. La durée de la bithérapie était variable en fonction du génotype viral : 24 semaines pour les patients infectés par un VHC de génotype 2 ou 3 et 48 semaines pour ceux infectés par un VHC de génotype 1 ou 4 . L'efficacité du traitement est évaluée par la quantification de l'ARN viral en fin de traitement et 6 mois après l'arrêt du traitement. La réponse virale prolongée (RVP) ou réponse virologique soutenue (RVS) est définie par un ARN viral indétectable en fin de traitement et 6 mois après l'arrêt du traitement, un patient rechuteur a une quantification de l'ARN viral indétectable à l'arrêt du traitement et détectable 6 mois après l'arrêt du traitement et un non-répondeur présente une quantification de l'ARN viral détectable pendant toute la durée du traitement. Avec la bithérapie (IFN $\alpha$-PEG + ribavirine), le taux de RVP était de 40 à $60 \%$ pour les patients infectés par un VHC de génotype 1 ou 4 alors qu'il est de $80 \%$ pour les patients infectés par un VHC de génotype 2 ou 3. Le traitement associant IFN $\alpha$-PEG et ribavirine présente des effets secondaires nombreux et très invalidants pour les patients. Enfin, cette bithérapie est sans effet chez les patients dont le foie est en stade de fibrose avancée ou à l'état de cirrhose. En 2011, deux inhibiteurs de la protéase NS3 du VHC ont obtenu l'AMM en France pour la prise en charge des patients infectés par un VHC de génotype 1 : le bocéprévir et le télaprévir. Ces inhibiteurs de protéase sont indiqués en association avec la bithérapie [IFN $\alpha$-PEG+ribavirine] aussi bien chez des patients naïs que chez des patients ayant déjà été traités par bithérapie seule. Les essais cliniques ont montré que pour les patients de génotype 1 , cette trithérapie permet d'atteindre des taux de RVP de l'ordre de $80 \%$ et de diminuer la durée totale des traitements de 48 à 24 semaines. Les schémas thérapeutiques varient en fonction de l'antiprotéase choisi et du statut du patient (naïf/en échec de bithérapie). Les schémas utilisant le bocéprévir démarrent par une période de bithérapie [IFN $\alpha$-PEG+ribavirine] avant la mise en place d'une trithérapie puis un retour ou non à la bithérapie, ceux utilisant le télaprévir commencent par la trithérapie avant de passer à une bithérapie. L'efficacité des protocoles thérapeutiques est évaluée par la quantification de l'ARN viral entre 8 et 12 ou 24 semaines de traitement. L'observance aux traitements est indispensable pour empêcher la survenue de mutations et de résistances. Le télaprévir et le bocéprévir doivent être pris 3 fois par jour, au cours de repas contenant des graisses. Le bocéprévir et le télaprévir sont métabolisés dans la voie du cytochrome P450 si bien qu'un traitement associé par des molécules empruntant cette voie doit être réévalué afin d'éviter les sur/sous-dosages. Les principaux effets secondaires du bocéprévir sont des troubles gastro-intestinaux (diarrhée, vomissements, hémorroïdes et dysgueusie) qui répondent aux traitements habituels et disparaissent à l'arrêt du traitement. Nous rapportons l'expérience de notre service sur une série de 12 patients en échec thérapeutique à une bithérapie initiale (patients rechuteurs), mis sous BOCEPREVIR en Octobre 2014 (disponible seulement en 2014)

\section{Prise en charge thérapeutique de la co-infection VIH/VHC}

$Z$ Boudiaf, A Mammeri, A Touref, ML Meghdecha, N Messalhi, M Laouar Service des maladies infectieuses CHU Annaba.

La coinfection VIH / VHC est fréquente du fait que les deux virus partagent certains modes de transmission. Cette co-infection est devenue un principal facteur de comorbidité et de mortalité en dehors du VIH. L'infection par le VIH aggrave le pronostic de l'infection par le VHC, avec une progression deux fois plus rapide de la fibrose et donc du risque de cirrhose, et représente, en cas d'immunodépression profonde, un facteur de risque d'évolution vers carcinome hépatocellulaire chez le jeune. L'instauration précoce du traitement antirétroviral est bénéfique car la restauration immunitaire et le contrôle de la réplication du VIH sous ARV ralentissent la progression vers la fibrose. Les personnes ayant un pourcentage de CD $4>25 \%$ et une Charge Virale VIH indétectable ont plus de chance d'atteindre une Réponse Virologique Soutenue (RVS) par rapport à celles dont le pourcentage de CD4 est plus faible et la 
réplication VIH n'est pas contrôlée. L'objectif principal du traitement anti VHC est d'obtenir une RVS définie par un taux d'ARN VHC indétectable 24 semaines après l'arrêt du traitement. Le traitement de référence de l'hépatite $C$ chronique pour les génotypes 2,3 reste l'association de PEG-IFN alpha et de Ribavirine et il doit être instauré quelque soit le stade de la fibrose et la charge virale. Chez les patients co-infectés par le VHC génotype 1 surtout et 4 , si le degré de fibrose est mineure (F0, F1), le traitement de l'hépatite $C$ pourra être reporté. En cas de fibrose modérée ou sévère, une trithérapie à base d'antiprotéases, comme le bocéprévir ou le télparévir associée au + Peg INF/ RBV est d'indication avec une prolongation de la durée du traitement anti-VHC à 72 semaines en fonction des caractéristiques pré-thérapeutiques: âge $>40$ ans, score METAVIR F3F4, charge virale VHC élevée; et de la réponse virale VHC à la semaine 4 et 12 . Les premiers essais chez les personnes co-infectées VIH-VHC naïves ont montré une proportion significativement plus élevée de RVS12 24 de la trithérapie par rapport à la bithérapie, et de bonnes réponses thérapeutiques précoces y compris pour des stades de fibrose avancée. Le traitement de l'hépatite C chez les patients VIH recevant des ARV nécessite une surveillance particulière du fait de fréquents problèmes d'intolérance et/ou d'interaction médicamenteuse. Les progrès thérapeutiques en matière de co-infection VIH VHC sont majeurs et le VIH n'est plus une limite à la guérison mais les principaux défis restent : le dépistage et l'accès au traitement pour le plus grand nombre de patients co-infectés.

\section{Hépatite virale Delta : diagnostic et traitement}

A Gasmi, W Guenifi, A Ouyahia, M Rais, A Hachani, S Mechakra, A Lacheheb

Service des Maladies infectieuses, CHU Sétif

L'infection VHD ne peut survenir que dans un contexte d'infection par le VHB. Les données recueillies, dix ans après la découverte du VHD (à la fin des années quatre-vingts) suggéraient que la prévalence du VHD dans le monde était de $5 \%$ des porteurs de l'Ag HBs, mais la distribution n'est pas uniforme. En Algérie, les seuls résultats disponibles remontent aux années quatre-vingt : E.H. Bellabesen 1985 à Alger a trouvé une séroprévalence de 7,5\% (9/120). Trente-sept ans après sa découverte, des points essentiels ont marqué l'épidémiologie du VHD. (1) Une diminution de la prévalence du VHD surtout dans les pays industrialisés. (2) Actuellement il est devenu clair que la prévalence du VHD à travers le monde n'est pas une simple réplique de celle du VHB. (3) Le risque d'hépatites Delta sévères similaires à celles signalées dans les années quatre-vingts en Amazonie est toujours présent dans les communautés pauvres du monde où l'infection par le VHB n'est pas contrôlée. (4) Le VHD ré-émerge dans plusieurs pays du monde où des fortes prévalences ont étés signalées. (5) La découverte de nouveaux génotypes ( 5 au 8) et tous en Afrique. Sur le plan clinique, deux cas de figures sont à distinguer : la Coinfection VHB et VHD et la Surinfection par le VHD chez un porteur chronique de l'Ag. HBs. Les tableaux cliniques de co-infections sévères très fréquentes au début de l'épidémie chez des jeunes sujets et décrites surtout en Amazonie et chez les usagers de drogues ont vu leur fréquence diminuée en faveur des hépatites chroniques chez des sujets plus âgés. On décrit actuellement beaucoup plus le VHD chez les cirrhotiques et ceux présentant de carcinomes hépatocellulaires. Le diagnostic sérologique d'hépatite delta ne peut être évoqué en première intention, que si L'Ag HBs est positif. Toutefois, la sérologie du VHB ne doit pas se limiter à la seule recherche de l' Ag HBs. Les IgM anti-HBc constituent le marqueur clé, ils permettent de distinguer une co-infection d'une surinfection. Le diagnostic indirect de l'infection par le VHD repose sur la recherche des anticorps du VHD dans le sérum par des méthodes radio-immunologiques ou mieux immunoenzymatiques. La détection de l'ARN du VHD est sans doute le meilleur examen à demander aux différents stades de l'infection pour confirmer le diagnostic et pour le suivi sous traitement. L'interféron- $\alpha$ (IFN- $\alpha$ ), molécule physiologique de défense contre les virus, est le seul traitement ayant montré une efficacité dans le traitement des hépatites $\mathrm{D}$ chroniques. Les analogues nucléos( $\mathrm{t}$ )idiques utilisés dans le traitement de l'hépatite virale B sont avérés inefficace. De nouveaux traitements sont actuellement en étude. Parmi ceux-ci, les inhibiteurs de prénylation sont prometteurs. Deux types d'action sont nécessaires pour la prévention de l'hépatite delta : la vaccination contre le VHB et l'application de mesures de réduction des risques de transmission.

L'évaluation non invasive du stade de fibrose reste une étape cruciale dans l'ère de traitements puissants des hépatites chroniques C (HCC) et B (HCB) Mona Munteanu

Unité de Diagnostic Non-invasif «Bilan Anti-Fibrose», Groupe Hospitalier Pitié-Salpêtrière, Paris. Unité de Recherche en Hépatologie, BioPredictive, Paris. Université Pierre et Marie Curie (UPMC), UMR_S938 INSERM UPMC, Paris, France

Grace aux panel de diagnostic sanguin non invasifs tels que FibroMax et l'élastographie unidimensionelle (Fibroscan) et shearwave (SWE, Aixplorer), la prise en charge des hépatites a été transformée dans les derniers années, de manière à ce que le patient aura dans moins de 20 minutes l'état exact de son foie, des informations pronostiques à long terme et la prédiction de la réponse au traitement. Malgré des traitements efficaces qui assurent un taux élevé de guérison virale (VHC) ou la suppression virale (VHB) et conduisent à l'amélioration du pronostic à long terme des patients, l'évaluation non invasive de la fibrose demeure importante. La classification initiale de la fibrose est essentielle d'un par pour le choix du traitement et de leur couts, d'autre part pour le dépistage de la cirrhose qui reste un facteur prédictif négatif indépendant pour la réponse virale soutenue (RVS). En présence de cirrhose, le taux de réponse et diminué malgré les options thérapeutiques de deuxième et troisième génération (Direct Acting Agents -DAA-thérapie). Des biomarqueurs sensibles comme le FibroTest restent extrêmement utiles chez les patients guéris pour suivre le risque résiduel de carcinome hépatocellulaire (CHC) estimé à environ $5 \%$ à 10 ans après la RVS. La présence de deux ou plusieurs facteurs métaboliques était prédictive du risque de non-régression de la fibrose après la guérison virale $\mathrm{C}$. Dans cette perspective, les biomarqueurs tels que le panel FibroMax ont démontré récemment leur efficacité pour le dépistage et le pronostic chez ces sujets avec risque restant de steatohépatite métabolique. Les marqueurs non invasifs de fibrose et d'activité (ActiTest) nous permettent d'identifier sans faire appel à la biopsie, les porteurs inactifs du VHB et de simplifier leur suivi. Chez les porteurs actifs du VHB, une étude récente avec FibroTest montre que, malgré la suppression efficace du virus par les analogues nucléos(t)idiques (NUC), l'incidence globale de la cirrhose augmentait, avec un risque résiduel de $90 \%$ avaient une progression de la fibrose identifiée lors d'évaluations répétées avec FibroTest. Des seuils prédéterminés du FibroTest et de l'élastographie ont été validés pour classer la gravité des cirrhoses en fonction du risque pronostique : F4.1-cirrhose sans décompensation, F4.2 - cirrhose avec varices sans décompensation et F4.3- cirrhose décompensée et/ou avec complications ( $\mathrm{CHC}$ ). En conclusion, les cliniciens doivent continuer à estimer la dynamique de la fibrose malgré la guérison virale (VHC) ou le contrôle virologique (VHB), car la fibrose peut évoluer dans près de $10 \%$ des patients ayant atteint la guérison ou un contrôle virologique efficace.

\section{Réversibilité de la fibrose hépatique}

A Benyahia, $S$ Tebbal

Service d'infectiologie, EPH Batna

Les hépatopathies chroniques entraînent, à plus ou moins long terme, la formation de fibrose dont le caractère latent et la progression lente au stade ultime de cirrhose compromettent souvent le pronostic vital. Les étiologies virales (VHB, VHC) en sont souvent la cause en raison de leur prévalence élevée. La fibrose est un processus dynamique résultant d'un déséquilibre entre production et dégradation de la matrice extracellulaire dont il est primordial d'en retarder la progression. Plusieurs études ont montré qu'après éradication de l'agent causal on observe, histologiquement, une stabilisation et également une régression de la fibrose. La réversibilité de la fibrose est étroitement liée à une viro-suppression prolongée et à la correction de cofacteurs aggravants. En pratique clinique, l'évaluation de la fibrose hépatique en particulier dans le suivi et en raison du caractère invasif de la biopsie, a été améliorée par le développement de méthodes non invasives (biomarqueurs, élastométrie). 
Traitement du diabète chez les patients atteints de cirrhose, une revue de la littérature.

S Rouabhia

Service de médecine interne, CHU de Batna.

Les liens entre le diabète et la cirrhose sont actuellement bien établis et confirmés par une littérature médicale très riche. Le diabète est présent chez plus de la moitié des patients cirrhotiques et de même le diabète de type 2 est souvent associé à une stéatose hépatique qui peut évoluer vers la cirrhose. Cette association fréquente cirrhose et diabète pose le problème de prise en charge. Le traitement du diabète chez les patients atteints de cirrhose est un sujet de débat, En effet il n'existe aucun consensus concernant la prise en charge des patients cirrhotiques diabétiques. Beaucoup de praticiens suspendent les antidiabétiques oraux chez leurs patients cirrhotiques diabétiques, du fait de leur toxicité hépatique potentielle. Cependant, malgré le métabolisme hépatique des antidiabétiques oraux, ils sont rarement hépatotoxiques. Ils peuvent être prescrits sans risque chez les patients cirrhotiques. L'insuffisance hépatocellulaire reste la seule contre indication de ces médicaments. La metformine possède de nombreux effets bénéfiques vis-à-vis du foie rapportés dans plusieurs études. Certaines études rapportent un risque accru du cancer du foie chez les patients traités par insuline. Dans cette conférence, nous faisons une revue de la littérature sur le traitement du diabète chez les patients diabétiques atteint de cirrhose. Un consensus entre diabétologues et hépatologues est indispensable pour une prise en charge optimale des patients diabétiques atteints de cirrhose.

\section{ABSTRACTS DES POSTERS THÉMATIQUES}

\author{
PA1- Hépatopathies toxiques d'origine professionnelle \\ I Mokrani ${ }^{1}$, I Miadi ${ }^{2}$ \\ ${ }^{1}$ Service de Médecine du travail EPSP Bouchegouf, Guelma; 2 Service
} d'Anesthésie Réanimation EPH Bouchegouf, Guelma.

Ce travail a pour objet de faire le point sur la littérature consacrée aux hépatopathies toxiques d'origine professionnelles afin de constituer un outil pratique utilisable pour aider au diagnostic. Les hépatopathies d'origines professionnelles sont en général non connues par les praticiens, pourtant de nombreux salariés sont exposés dans le cadre du travail à des produits chimiques susceptibles d'être toxique pour le foie. Le problème pour le médecin généraliste ou spécialiste est de pouvoir faire la preuve de l'origine professionnelle; D'où l'intérêt d'une collaboration étroite entre médecins généralistes, hépato-gastroentérologues et médecins du travail.

PA2- Vingt-cinq ans de Vaccination contre l'hépatite virale B: bilan et contraintes

Az Nezzal, S Mélais, D Khezzane, Am Nezzal

Service de Médecine du travail, Faculté de Médecine, Université Badji Mokhtar, Annaba.

Le risque d'hépatite virale $B$ constitue le «Risque » le plus fréquent chez le personnel de santé. La vaccination contre l'hépatite $B$, préconisée pour le personnel de santé depuis 1988, a été rendue obligatoire depuis 2000 en Algérie. C'est ainsi que le personnel du CHU de Annaba a bénéficié d'un programme de vaccination contre l'hépatite virale B depuis 1988. Celui-ci nécessite des évaluations quantitatives et qualitatives périodiques. Objectifs : Décrire les caractéristiques épidémiologiques de la population à vacciner. Evaluer le programme de vaccination. Matériels et méthodes : Type d'étude: étude descriptive rétrospective. Population d'étude: 4471 travailleurs. Matériels de l'étude : Fichiers de la vaccination, documentation et réglementation. Variables de l'étude: âge, sexe, profession, catégorie professionnel, service, structure et statut vaccinal. Résultats : Plus $50 \%$ des travailleurs ont un âge supérieur à 40 ans et $59 \%$ des travailleurs sont de sexe féminin. $30,1 \%$ et $31 \%$ des salariés appartiennent respectivement aux corps paramédical et technique. Le corps médical salarié représente environ $12 \%$ de l'effectif global du CHU d'Annaba. 87,9\% des travailleurs, corps médical non inclus, sont correctement ou en cours de vaccination. Les résidents avec le personnel médical salarié, avec une couverture vaccinale respectivement de $33 \%$ et $14 \%$, constituent les deux populations les moins vaccinées. Conclusions : La vaccination en milieu de travail, en particulier la vaccination contre l'hépatite virale B, constitue une activité importante du service de Médecine du travail. Les contraintes subies par le programme de vaccination constituent des axes d'amélioration du programme de vaccination contre l'hépatite virale $B$.

PA3- Négativation de l'Ag HBS après 36 semaines de traitement par Peg interféron alpha 2 a d'une hépatite $B$ chronique à AgHBe négatif : à propos d'un cas

S Boulanouar ${ }^{1}$, M.L Meghadecha ${ }^{2}$

${ }^{1}$ Service d'infectiologie, EPH Ibn Zohr Guelma

${ }^{2}$ Université Badji Mokhtar, CHU Annaba

Contexte : L'hépatite B est une maladie virale cosmopolite posant un problème de santé publique en Algérie, où elle prédomine dans l'est du pays. C'est une pathologie qui pose encore un problème thérapeutique, l'Ag HBS reste présent dans le sang après le traitement par peg interféron ou entécavir. Nous rapportons une négativation de l'AgHBs après 36 semaines de traitement par peg interféron alpha 2a. Présentation du cas : Monsieur H.S âgé de 26 ans, dont l'hépatite B est découverte chez lui en 2011 lors d'un don de sang et ayant comme facteur de risque des soins dentaires réalisés quatre mois avant la découverte de l'infection. L'exploration initiale avant le traitement a objectivé un ADN du virus de l'hépatite $\mathrm{B}(\mathrm{VHB})=2707 \mathrm{UI} / \mathrm{ml}$ soit $(3,43 \mathrm{log})$ calculé par PCR temps réel, Alanine transférase (ALT) $=30 \mathrm{UI} / \mathrm{l}$, un AgHBe négatif, l'exploration histologique du foie par biopsie hépatique trouvant un score Metavir A1F2. Le patient était mis sous Peg-Interferon alpha 2a pendant 48 semaines à partir du mois de Mai 2014 avec un suivi régulier pour juger de l'efficacité du traitement et de la survenue des effets secondaires. Le traitement était bien toléré dans l'ensemble, on a noté un syndrome pseudo grippal ayant rapidement cédé après la quatrième semaine. Sur le plan efficacité on notait une négativation de l'ADN VHB à la $12^{\text {ème }}$ semaine de traitement, ainsi qu'à la 24ème et à la 48ème semaine. Une augmentation des ALT était observée du troisième au cinquième mois puis la réponse biochimique était complète et durable avec ALT inferieur à $40 \mathrm{UI} / \mathrm{l}$. Sur le plan immunologique, une négativation de l'Ag HBS était survenue à la $36^{\text {ème }}$ semaine de traitement avec appariation des Anticorps antiHBs avec un titre de 66UI/ml. Discussion : L'interféron à l'heure actuelle offre les meilleures chances de perte de l'Ag HBS surtout si l'ADN du VHB est négative sous et après la fin traitement. Conclusion: L'hépatite B est une maladie virale cosmopolite qui pose un problème de santé publique dans notre pays, elle pose aussi un problème thérapeutique, l'AgHBS reste présent dans le sang malgré un traitement adéquat, mais le cas clinique rapporté ci-dessus représente un succès thérapeutique avec une perte de l'Ag HBS dans la 36 ème semaine de traitement.

PA4- L'évolution favorable d'une hépatite $B$ nosocomiale posttransfusionnelle : a propos d'un cas.

H Sbaghdi, ML Meghadecha, M Aidaoui, A Mammeri, M Laouar Service d'infectiologie, Dr Dorban CHU Annaba.

L'hépatite virale constitue un problème majeur de santé publique, du fait quelle soit associée à une mortalité et une morbidité non négligeables, le virus de l'Hépatite $\mathrm{B}(\mathrm{VHB})$ en est la première cause. Dans le monde, on estime qu'environ 350 millions de personnes sont chroniquement infectées par le virus de l'hépatite $B$ et 1 million meurent chaque année des conséquences de cette affection. L'hépatite $\mathrm{B}$ se transmet par exposition cutanéo-muqueuse à du sang ou d'autres produits biologiques infectés par le virus. Les principales voies de transmission sont : sexuelle, sanguine et mère-enfant. L'infection par le VHB conduit à la guérison dans $90 \%$ cas, sa gravité dans la phase aigue est due à la forme fulminante. Le diagnostic de certitude de l'hépatite virale B repose sur la sérologie, alors que la prévention se fait par la vaccination. Nous présentons ici le cas d'un patient de 24 ans atteint d'une hépatite $B$ dans sa forme aigue grave ayant comme facteur de risque une poly transfusion sanguine pour une drépanocytose homozygote $S / S$, pour laquelle le diagnostic été posé précocement à la phase aigue et l'évolution été favorable vers la guérison. Ce cas clinique témoigne de la persistance à l'heure actuelle d'une contamination nosocomiale post transfusionnelle d'où la nécessité d'un meilleur contrôle au niveau des banques de sang 
notamment par l'introduction dans le dépistage du sang des Ac HBc voire même de la PCR du virus de l'hépatite B pour minimiser au mieux le risque de transmission due à la fenêtre sérologique, toutefois quelque soit les efforts fournis le risque zéro n'existe pas et les transfusions sanguines doivent être limitées aux vrais indications.

\section{PA5- Caractérisation et identification de cryoglobulines} associées aux hépatites virales.

A Alliouch-Kerboua ${ }^{1}, S$ Gadiri-Meriche ${ }^{1}, H$ Meriche $^{1}, Z$ Boumaza ${ }^{2}$, A Trima $^{2}, M_{\text {Laouar }}^{2}$

${ }^{1}$ Service d'Immunologie, Clinique Sainte Thérèse, CHU Annaba, Annaba, Algérie. ${ }^{2}$ Service d'infectieux, Hopital Dorban, CHU Annaba, Annaba, Algérie.

Introduction : Les cryoglobulines sont des immunoglobulines ou des complexes immuns qui précipitent à froid et se redissolvent à $37^{\circ} \mathrm{C}$. Elles peuvent accompagner des hémopathies, des maladies autoimmunes, des infections, notamment l'hépatite $\mathrm{C}$ chronique. Elles sont définies par leur typage immunochimique selon la classification de J.C. Brouet et celle qui résulte de sa révision par D. Le Carrer : type I (immunoglobulines monoclonales), type IIa (immunoglobuline monoclonale associée à des immunoglobulines polyclonales), type IIb (immunoglobuline monoclonale associée à des immunoglobulines oligoclonales) et type III (immunoglobulines polyclonales). Seules les cryoglobulines de type II et III (mixtes) sont associées à l'infection par le virus de l'hépatite $\mathrm{C}$. Le but de ce travail est de rapporter les résultats du protocole de recherche et d'identification établi par le laboratoire d'Immunologie pour les cryoglobulinémies mixtes liées aux hépatites virales C. Matériel et méthodes: Le présent travail a été réalisé sur 30 sérums de patients ayant une hépatite virale C (14 femmes et 16 hommes), la moyenne d'âge était de 44 ans. La détection de la cryoglobuline et sa caractérisation a fait appel à des techniques immunologiques telles que : l'électrophorèse sur gel d'agarose et l'immuno-diffusion double (technique d'Ouchterlony) qui sont des tests sensibles et spécifiques. Résultats: Cette étude a mis en évidence la présence de cryoglobulines chez 33,33\% des patients infectés par le virus de l'hépatite C. Le typage immunochimique de ces cryoglobulines a montré qu'elles sont de type III. Conclusion : Au terme des résultats préliminaires de notre étude, nous concluons que les cryoglobulines mixtes sont fréquemment associée à une infection par le virus de l'hépatite $\mathrm{C}$. La détection et l'identification de ces complexes immuns est importante pour la surveillance de possible complications viscérales et le diagnostic des manifestations extra hépatiques de l'infection par le virus de l'hépatite $\mathrm{C}$.

PA6- Est-ce que la vaccination introduite dans le calendrier vaccinal algérien contre le virus de l'hépatite $B$ est efficace ? $N$ Touhami, ML Meghadecha, M. Aidaoui, M. Laouar

Service d'infectiologie, CHU Annaba

Contexte : L'hépatite $B$ chronique est un problème de santé publique en Algérie, vue la morbidité et la mortalité résultante, particulièrement dans les zones à forte endémicité où l'infection touche les plus jeunes principalement par transmission maternofœtale avec risque élevé de cirrhose et de carcinome hépatocellulaire. En Algérie, la vaccination contre le VHB (virus de l'hépatite B) a été introduite dans le programme vaccinal en décembre 2002, le schéma comporte 3 injections à $\mathrm{M} 0-\mathrm{M} 1-\mathrm{M} 6$ avec une vaccination dès la naissance. Le but de ce travail est de calculer la prévalence des Ac anti HBs développés chez des enfants vaccinés contre le VHB, le titre de protection retenue est de $10 \mathrm{UI} / \mathrm{l}$. Matériel et méthode : Il s'agit d'une étude rétrospective et descriptive transversale à visé de séroprévalence. On a inclus dans notre étude 81 enfants dont l'âge varie entre 9 mois et 11 ans, correctement vaccinés contre le VHB suivant le calendrier vaccinal algérien, sur une période s'étalant sur une période de deux ans du 01/01/2013 au 31/12/2014, réalisée au niveau de la consultation de l'hépatite $B$ du service d'infectiologie du CHU d'Annaba, dans le cadre du dépistage de l'entourage familiale d'un patient porteur d'un antigène HBs positif. On été exclus de cette étude les enfants non correctement vaccinés, les enfants porteurs d'un AgHBs+, et les enfants ayant eu un contact avec le VHB et guéris (AcHbc positif). Résultats : La moyenne d'âge était de 4 ans avec des extrêmes allant de 9 mois à 11 ans, le sexe ratio $0,92.62 \%(n=50)$ des enfants étaient immunisés parmi lesquels seuls $42 \%$ présentaient un taux d'AcHBs très satisfaisant supérieur à 100UI/l contre 58\% entre 10 et 100UI/l. 38\%(n=31) n'étaient pas protégés avec un titre des Ac anti Hbs <10UI/l. Conclusion: Selon les résultats obtenus, l'efficacité du vaccin anti VHB semble suboptimale, le rajout de la 4ème injection de rappel dans le nouveau calendrier vaccinal permettrait d'optimiser l'immunogènicité du vaccin et sa fiabilité.

\section{PA7- Bevacizumab : effets thérapeutiques et toxiques} W Soudani ${ }^{1}$, R Djafer ${ }^{2}$, H Djeddi ${ }^{3}$, S Boughrira ${ }^{3}$, F Griffi ${ }^{3}$

${ }^{1}$ Laboratoire de Chimie thérapeutique,Département de Pharmacie, Faculté de Médecine Annaba. ${ }^{2}$ Service de Toxicologie CHU Annaba. ${ }^{3}$ Service d'hémato- oncologie CHU Annaba.

Introduction : L'utilisation des anticorps chimériques humanisés ou humains dans le traitement du cancer dont le but est d'assurer un meilleur contrôle des effets secondaires, permet aujourd'hui de noter des résultats prometteurs non seulement dans le traitement des tumeurs hématologiques mais aussi dans le traitement de certaines tumeurs solides. Nos objectifs étaient l'évaluation de la tolérance au Bévacizumab chez les patients cancéreux et l'influence des associations cytotoxiques sur cette tolérance, ainsi que la détermination des principaux facteurs de risque cancérigènes chez ces patients. Matériel et Méthode : Nous avons réalisé une étude rétrospective descriptive sur le traitement par le Bévacizumab à partir d'une enquête chez cinquante (50) patients cancéreux suivis au niveau du service d'hémato- oncologie CHU Annaba, prédominés par les femmes âgées de 45 à 85 ans, dans la période allant du mois de Décembre 2013 au mois de mai 2014. Résultats et Discussion: L'exposition prolongée aux certains facteurs endogènes ou exogènes pouvait augmenter le risque d'avoir un cancer à savoir le facteur hormonal (inducteurs d'ovulation) chez la femme $14 \%$ et le tabac $6 \%$. Les principales toxicités spécifiques liées au bévacizumab étaient l'hypertension artérielle $(27,27 \%$ en monothérapie, $30,67 \%$ en bithérapie, 7,69 \% en trithérapie) et l'hémorragie ( $45,45 \%$ en monothérapie, $23,07 \%$ en bithérapie, $15,38 \%$ en trithérapie) tandis que l'asthénie, les effets digestifs et les troubles neuropsychiques étaient liés aussi bien au bévacizumab qu'auxcytotoxiques. Nous avons démontré l'influence de la chimiothérapie associée sur l'augmentation de la fréquence des effets indésirables spécifiques, communs et cumulatifs. Nous avons constaté l'influence de la chimiothérapie sur la nature des effets indésirables cumulatifs produits par le Bévacizumab, des effets cumulatifs plus graves ont été obtenus en bithérapie (des troubles de la vision, des douleurs osseuses, des épigastralgies, fibrose pulmonaire, diabète chez 7.69\%) et en trithérapie (l'anémie chez $23.07 \%$, le diabète chez $15.38 \%$, le caillot du sang et l'épigastralgie chez 7.69\%). Conclusion :La nature et la fréquence des effets indésirables développés suggère la nécessité de solliciter les autorités de santé responsables à améliorer la dispensation de médicaments anticancéreux surtout les thérapeutiques ciblées chères, et de différents moyens anticancéreux notamment la radiothérapie, afin d'assurer une meilleure prise en charge des cancéreux.

PA8- La prévalence des hépatites virales $\mathrm{B}$ et $\mathrm{C}$ au niveau des CHU d'Annaba.

M Bouzrara, K Amoura, M Dekhil.

CHU Annaba

Les hépatites virales posent un problème mondial de santé public. Dans le but de déterminer la séroprévalence des hépatites virales $B$ et $\mathrm{C}$, nous avons réalisé une étude rétrospective au niveau du laboratoire de microbiologie CHU Annaba, La durée de l'étude est étendue du mois janvier 2013 à décembre 2014. La population étudiée : patients hospitalisés et externes. La méthode utilisée pour le diagnostic : ELISA. Le nombre de tests pour le dépistage de l'hépatie virale C dans l'année 2013 est 5614 dont 211 sont positifs, ce qui donne une prévalence de $3,758 \%$, Le nombre de tests dans l'année 2014: 6321 dont 238 sont positifs, ce qui donne une prévalence de : $3,765 \%$. La prévalence chez les externes est $0,168 \%$ avec un ratio : $\mathrm{H} / \mathrm{F}=0,41$. La tranche d'âge la plus touchée par HCV est de (20 à 50) ans. Le nombre de tests pour l'hépatite virale B dans l'année 2013 est de 5833 dont 100 sont HBV positifs, ce qui donne une prévalence de : $1,71 \%$, le nombre de tests dans l'année 2014 est de 7010 avec 233 test positif, ce qui donne une prévalence de 3,32\%. la prévalence chez les externes est de $0,17 \%$ avec un sexe ratio $\mathrm{H} / \mathrm{F}=1,5$, la tranche d'âge la plus touchée par l'HBV est de (20 à 50) ans. La majorité des patients détectés comme atteints de l'hépatite $\mathrm{C}$ 
sont hospitalisés au niveau des services de maladies infectieuses et d'hématologie et de néphrologie. En conclusion, on remarque une augmentation de l'incidence de HBV, le problème est lié peut être a une vaccination insuffisante,surtout la population a haut risque (les polytransfusés , les insuffisant rénaux , les thalassémiques ....)

\section{PA9- Effet hépatoprotecteur d'un antioxydant naturel extrait du thé vert}

A Labdi ${ }^{1}$, A Bitam $^{1}$, EA Koceir ${ }^{1}$, C Belarbi $^{1}$, A Zouambi $^{3}$, S Zabel $^{2}$

1 Equipe de Bioénergétique et Métabolisme Intermédiaire, LBPO/FSB/USTHB, Alger ${ }^{2}$ Laboratoire biotechnologie, /FSA/UHB, Chlef ${ }^{3}$ Laboratoire d'histopathologie, Ecole National Vétérinaire d'Alger

Objectif: Cette étude s'inscrit dans la perspective d'une évaluation de l'effet hypocholestérolémiant et hépatoprotecteur des polyphénols extraits du thé vert( Camellia sinensis L.). Matériel and méthodes: Ce travail a été commencé par l'extraction des polyphénols des feuilles du thé vert (Camellia sinensis) par décoction. Les teneurs en composées phénoliques et en flavonoïdes et l'activité antioxydante de l'extrait du thé vert ont été évaluées par la spectrophotométrie (UV). L'expérimentation animale a été réalisée en cinq mois ( Mai - Octobre 2012)à l'animalerie de USTHB d'Alger. Des rats Wistar (Rattus norvegicus) sains de sexe masculin ont été fournis de l'Institut Pasteur d'Alger ; ils ont été répartis au hasard en trois groupes expérimentaux (groupe normal « $\mathrm{T} »$, groupe de contrôle "gavage de cholestérol (1.5\%) « $\mathrm{C}$ » et groupe curative "CG", $(\mathrm{n}=6)$. Les profiles lipidiques qui ont été réalisées (cholestérol total "CT", triglycérides "TG", lipoprotéine de haute densité (HDL-Chol); lipoprotéines de basse densité( LDL-Chol) et lipoprotéines de très basse densité (VLDL-Chol). Histopathologie;une coloration de routine a été effectuée sur des lames en utilisant deux colorants différents hématoxyline-éosine (HE) combine hématoxyline qui colore les noyaux pourpre et éosine le cytoplasme rose. Résultats : L'extrait du thé vert est très riche en polyphénols « catéchines) et en flavonoïdes dont leurs teneurs sont respectivement $(29,5 \%$ et $25,6 \%$ de l'extrait du thé vert), ces derniers révèlent une activité antioxydante puissante avec un pourcentage de réduction de $70 \%$. Après quatre semaines d'expérimentation, les taux plasmatiques de LDL-C TC et TG ont augmenté significativement $(\mathrm{p}=0,05)$ dans les groupes nourris $1,5 \%$ de cholestérol (C,CG) par rapport au groupe normal (T), mais le taux plasmatique de HDL-Chol a été diminué. Après 8 semaines de traitement, il y a une diminution très hautement significative $(p=0,05)$ du taux plasmatique des LDL-Chol et des VLDLChol dans le groupe «CG» administré oralement par gavage les polyphénols du thé vert par rapport au groupe « $C$ », tandis que les taux plasmatiques de TC,de LDL-C et de VLDL-C ont diminué par rapport au groupe $« \mathrm{C} »$. Pour le groupe contrôle $« \mathrm{C} »$ les résultats obtenus sont prouvés histologiquement dans le foie par la présence d'une stéatose non alcoolique micro et macro vacuolaire avec la présence aussi de beaucoup de corps de Mallory. Tandis que le groupe curatif qui a subit le traitement par notre extrait, a eu une régénérescence importante des cellules hépatiques qui se traduit par une présence de cellules binucléaires (division mitotique). Conclusion: Le thé vert est l'une des boissons les plus populaires à travers le monde. Il fournit également une source alimentaire de composés biologiquement actifs qui aident à prévenir une grande variété de maladies. Les catéchines du thé vert qui donnent ses qualités anti-oxydantes sont également actives dans la protection du foie.

PA10- Lipoprotéine $X$ et Cholestase extra et intra hépatique (hépatite B et C).

H Benghezel

Hôpital non universitaire, EPH djillali rahmouni laboratoire, Alger, Algeria

Le but étant La mise en évidence d'une augmentation de la lipoprotéine $\mathrm{X}$ secondaire à des cholestases intrahépatiques comme les hépatites $B$ et $C$ ou extra hépatiques en éliminant les patients qui présentent une anomalie du métabolisme glucidique ainsi que ceux qui présentent un trouble héréditaire du métabolisme lipidique à été vérifier par le dosage du cholestérol / triglycérides selon la méthode de TRINDER des TGO/TGP/GGT Selon la méthode IFCC $37^{\circ} \mathrm{C}$ La phosphatase alcaline selon la méthode DGKC $37^{\circ} \mathrm{C}$ sur automate
ERBA XL 200. Sur un échantillon de 78 patients présentant une cholestase admis en service de gastro entérologie de différentes étiologies notamment d'hépatite $\mathrm{B}$ et $\mathrm{C} 32$ patients présentent un bilan lipidique perturbé, $83 \%$ des 32 patients présentent un taux de cholestérol $>2,40 \mathrm{~g} / \mathrm{L}$ alors que seulement $27 \%$ présentent un taux de triglycérides $>1,60 \mathrm{~g} / \mathrm{L}$. La présence d'un taux élevé de cholestérol constitue un témoin indirect de la présence de la lipoprotéine X (lp-X) cette dernière est mise en évidence par électrophorèse, c'est une particule lamellaire de 30 à $70 \mathrm{~nm}$ de diamètre elle est caractérisée par sa haute teneur en phospholipides (de $66 \%$ en poids) et en cholestérol non estérifié (22\%), et sa faible teneur en protéines, en esters de cholestérol et en triglycérides, elle ne contient pas d'apolipoprotéine B et donc elle n'est pas éliminé par le récepteur du LDLc, la Lp X disparaît généralement peu après que la cholestase (hépatite) soit guérie. En conclusion La Lipoprotéine -X peut être trouvée dans les échantillons de sérum de patients atteints de cholestase bien que sa présence ne permet pas de faire la distinction entre les causes intra hépatique (hépatiteB et $\mathrm{C}$ ) et extra-hépatiques.

PA11- Hépatite et addictologie : possibilités et limites de la prise en charge psychiatrique

W Fetni ${ }^{1}$, H Chirouf ${ }^{2}$

${ }^{1} \mathrm{EPH}$ Ibn Zohr, Guelma. ${ }^{2} \mathrm{EPH}$ Bouchaguof, Guelma

Prendre en charge un malade soufrant d'un phénomène d'addictologie est une chose difficile, mais il serai plus délicat quand cette prise en charge est destinée à un malade souffrant d'une hépatite grave avec un syndrome de cytolyse important, sans oublier que ce même patient présente une personnalité pathologique qui rend les choses plus difficiles encore. Penser à former des équipes multidisciplinaires pour la prise de cette pathologie lourde devient une nécessité de santé publique vu l'ampleur et la gravité de la toxicomanie dans notre société. Ce cas de figure est illustré par l'étude d'un cas clinique de notre pratique quotidienne d'interservice (maladies infectieuses et psychiatrie).

PA12- Hépatite C et Dysthyroïdie : Effet de l'infection ou du traitement?

A Gouri ${ }^{1}$, A Dekaken 2 , M Chefrour ${ }^{3}$, AA Bentorki ${ }^{1}, M$ Bouchareb $^{1}, F$ Mahiddine $^{1}$, M Belleili ${ }^{4}$, A Yakhlef $^{1}$

1 Laboratoire de Biochimie Clinique, EPH Ibn Zohr, Guelma, 2 Laboratoire de Biochimie. Hôpital de La Timone Enfant, Marseille, France, ${ }^{3}$ Service de Médecine Interne, EPH El Okbi, Guelma, ${ }^{4}$ Laboratoire de chimie analytique et pharmaceutique, Faculté de médecine, Annaba

La dysthyroïdie représente une des plus fréquentes manifestations endocriniennes de l'infection par le virus de l'hépatite C (VHC), exacerbée par le traitement à base de l'interféron. Le spectre des anomalies va de la production isolée des anticorps antithyroïdiens à l'hypothyroïdie, maladie de Basedow et la thyroïdite destructrice. L'objectif de cette mise au point est de passer en revue les différents aspects biologiques et physiopathologiques de cette association. En fait, la relation entre infection par le VHC et maladies thyroïdiennes soulèvent plusieurs questions : la prévalence de l'auto-immunité thyroïdienne chez les patients atteints d'hépatite chronique $C$, la prévalence de l'infection par le VHC chez les patients atteints de maladies thyroïdiennes auto-immunes, les effets sur la fonction thyroïdienne du traitement par interféron alpha conduit pour hépatite chronique $\mathrm{C}$. Si le traitement de l'hépatite $\mathrm{C}$ par interféron alpha peut se compliquer de dysthyroïdie, le lien direct entre dysthyroïdie et infection par le VHC reste à discuté. Les résultats de la plupart des études confirment une prévalence plus élevée des troubles auto-immunes de la thyroïde et de l'hypothyroïdie chez les patients porteurs chroniques du VHC. Le sexe féminin et la présence des anticorps anti TPO/Anti microsomes sont respectivement les principaux facteurs de risque pour le développement de l'hypothyroïdie et la dysthyroïdie auto-immune. Cependant, aucune étude n'a montré un changement significatif de la prévalence de l'hyperthyroïdie chez les sujets infectés par le VHC. Des études plus récentes ont trouvé que l'interféron alpha induit dans 3,5 a $15 \%$ des cas des dysthyroïdie dont les formes cliniques sont extrêmement diverses. I1 s'agit dans deux tiers des cas d'hypothyroïdies et dans un tiers des cas d'hyperthyroïdies.

En conclusion, des études cliniques sont encore nécessaires pour, d'une part, mieux préciser les relations entre infection par le VHC et 
auto-immunité thyroïdienne et, d'autre part, mieux définir les facteurs prédictifs de survenue de dysthyroïdie au cours des traitements par interféron alpha.

\section{PA13- Évaluation du programme de dépistage de l'HVB, l'HVC et de HIV \\ Az Nezzal, S Mélais, D Khezzane, M Douadi, Am Nezzal}

Service de Médecine du travail, Faculté de Médecine; Université Badji Mokhtar, Annaba

Le risque de transmission de l'HVB, l'HVC et de l'HIV lors des soins est un problème connu des professionnels de la santé. Même s'il est rare, il n'est pas exceptionnel. Donc le risque existe. L'OMS recommande que chaque pays doit évaluer ses performances de dépistage, car il représente un moyen nécessaire et efficace pour la lutte contre ces infections. La recherche simultanée des marqueurs sérologiques de ces infections (Ag HBs, Ac Anti-HVC et AcAnti-HIV) est une des stratégies de dépistage utilisée en particulier en Algérie. Objectifs : 1 . Décrire la population soumise au programme de dépistage en milieu hospitalier. 2 . Evaluer le programme de dépistage ('HVB, HVC et de HIV) sur une période de 06 ans. Matériels et méthodes : Type d'étude: descriptive rétrospective (2009 au 2014). Lieu de déroulement de l'étude : CHU. Durée de l'étude : 04 mois. Population d'étude: 2140 personnes. Matériels de l'étude : Registre des prélèvements, registre des maladies professionnelles, fichiers de vaccination contre l'HVB. logiciel Excel. Variables de l'étude: Age, sexe, profession, catégorie professionnelle, structure, année des prélèvements, statut vaccinal et les résultats des examens sérologiques. Outils statistiques: mesures de fréquence. Résultats : Sur 2140 sujets constituant la population d'étude, 4542 prélèvements ont été réalisés entre 2009 et 2014. Cette étude révèle que le dépistage de ces infections reste très faible (HVB et HVC) voire nul (HIV) dans la population surveillée. Conclusions : Cette évaluation de la stratégie de dépistage et de ses performances nous impose de revoir la stratégie mise en place depuis plus d'une vingtaine d'années pour lutter efficacement contre ces infections.

PA14- Les nouveaux traitements de l'hépatite $c$; une action multi-génotypique, effets indésirables moindres, succès thérapeutiques, au cours de développement des médicaments biosimilaires et biologique

A Belhachem, A Merah, H Houamria, $S$ Bouledroua, M Guidoum, $M$ Rezzag Bara, A Benoumechiara, C Krid, K Bourdjiba, .A Haji

Les biomédicaments sont indiqués pour le traitement d'un nombre croissant de pathologies. Ils constituent un axe de développement majeur pour l'industrie. Actuellement, plus d'un nouveau médicament sur deux autorisé en Europe est un biologique. Les biosimilaires ne sont pas les génériques des médicaments de biotechnologie, comme on les présente parfois. Il s'agit au contraire d'une nouvelle catégorie à part entière de médicaments qui ne sont ni radicalement différents ni radicalement identiques aux produits dont ils sont la copie légale, et dont l'apparition sur le marché soulève des questions techniques, législatives et économiques inédites. En 2020, les analystes de IMS Health prévoient un CA mondial des biosimilaires compris entre 11 et 25 milliards de dollars, en fonction de la dynamique, encore inconnue, du marché nord-américain. Cela représenterait entre 4 et $11 \%$ du marché total des biologiques à cet horizon de temps. En supposant un différentiel de prix de l'ordre de $25 \%$, les biosimilaires pourraient entraîner une baisse des dépenses pharmaceutiques globales de l'ordre de 15 à 30 milliards de dollars par an. L'épidémie de VHC au total, 185 millions de personnes sont infectées par le VHC dans le monde. On estime que les maladies du foie liées au VHC provoquent le décès de 350000 personnes chaque année. Actuellement, la bithérapie Peg-interféron (PEG-IFN), administrée par injection, et Ribavirine (RBV), est le traitement leplus utilisé. Le taux de succès thérapeutique varie de 50 à $80 \%$, et le traitement s'accompagne d'effets indésirableslourds et multiples. L'année 2014, c'est le tournant majeur dans l'histoire de la pandémie. Les nouveaux traitements, récemment mis sur le marché ou devant l'être prochainement, présenteraient de nombreux avantages par rapport à leurs prédécesseurs : une action multi-génotypique, des effets indésirables moindres et de bien meilleurs taux de succès thérapeutiques, y compris chez les personnes se situant à un stade avancé de l'infection. Une autorisation de mise sur le marché a été accordée en novembre 2013 par l'agence européenne du médicament(EMA) et en décembre 2013 par l'agence américaine des produits alimentaires et pharmaceutiques (FDA) à l'inhibiteur de lapolymérase nucléotidique/AAD sofosbuvir (SOF), récemment mis au point par Gilead. le taux de succès thérapeutique dusofosbuvir est proche de $90 \%$ selon les résultats des essais cliniques réalisés jusqu'ici. Le simeprevir de Janssen a également fait l'objet d'une autorisation de mise sur le marché de la FDA en nombre 2013. Le laboratoire Bristol-Myers Squibb(BMS) a soumis le daclatasvir à la FDA ; la demande est en cours. Le sofosbuvir sera très probablement suivi d'autres AAD, commercialisés par AbbVie, Janssen et BMS.

PA15- Hépatite $A$ aigue dans sa forme cholestatique : à propos d'un cas

S Boufarou, N Messalhi, ML Meghadecha, A Trima, M Laouar Service d'infectiologie, CHU Annaba

Introduction : L'hépatite virale A est une maladie infectieuse due à un virus hépatotrope, ubiquitaire et survenant de manière sporadique ou épidémique. La contamination se fait par contact direct d'une personne infectée, ou indirectement par consommation d'eau, ou d'aliments contaminés contenant des virus excrétés dans les fèces de personnes infectées. L'hépatite $\mathrm{A}$ dans sa forme cholestatique est moins fréquente que la forme cytolytique. La cholestase prédomine et la cytolyse hépatique est discrète. L'évolution de ces formes même graves est généralement lente en 3 à 4 mois, mais la guérison est habituellement complète. But : décrire une entité clinico-biologique une hépatite $A$ dans sa forme cholestatique. Présentation du cas: Patient âgé de 17 ans, sans antécédents pathologiques particuliers, admis au service d'infectiologie CHU Annaba le 30/03/2014 pour la prise en charge d'un ictère cutanéo-muqueux généralisé franc avec un TP bas à $36 \%$ et un taux de facteur $\mathrm{V}>60 \%$ évoluant depuis deux mois. Sur le plan biologique un syndrome de cholestase été prédominant avec une discrète cytolyse et absence d'insuffisance hépatocellulaire. Une sérologie d'hépatite A type IgM revenant positive. Le test de koller été positif. Le patient a bien évolué cliniquement avec régression de l'ictère et biologiquement avec augmentation progressive du TP. Conclusion: Le présent cas décrit une forme cholestatique d'hépatite $A$, souvent négligée induisant un retard diagnostique, et faisant suspecté même l'existence d'une forme grave. L'évolution de ces formes reste habituellement favorable et complète.

PA16- Chimio prophylaxie de la réactivation de l'hépatite $B$ au cours de immunosuppression : à propos d'une observation H Brahimi, S Benchouk, A Benabdellah

Service des maladies infectieuses, CHU Tlemcen

Le virus de l'hépatite $B$ est une cause importante de réactivation virale d'hépatite chez les patients immuno-supprimés. Les facteurs de risque sont l'antigène HBs positif, un traitement par rituximab, une pathologie onco-hématologique ou encore une transplantation de moelle, néanmoins l'introduction d'un traitement prophylactique antiviral est indiquée dans les cas à haut risque de réactivation virale $\mathrm{B}$ et elle diminue la morbidité et la mortalité liées à une poussée d'hépatite. Il s'agit d'une femme de 71 ans, aux antécédents de diabète type II insulino-nécessitant, HTA et goitre multi nodulaire toxique. Le début des symptômes était progressif, marqué par l'apparition d'un syndrome digestif fait d'Épigastralgies, nausées, anorexie et méléna avec une pâleur cutanéomuqueuse extrême. Les explorations ont conduit au diagnostic de lymphome non hodgkinien digestif à grandes cellules de haut grade de type $B$ (antropyloroduodénal) avec des infiltrations pancréatiques et des vaisseaux mésentériques. L'hépatite virale $B$ était de découverte fortuite avec un Ag HBs positif, Ag HBe négatif, Ac anti HBc (IGG) positif et Ac anti HBe positif ainsi qu'une charge virale initiale indétectable. Un traitement antiviral à base d'entécavir a été instauré simultanément avec une chimiothérapie «RCHOP» de six mois, relayée par Rituximab pendant 02 ans, néanmoins ce traitement antiviral a été poursuivi encore douze mois après la fin de l'immunosuppression et dont l'efficacité a été prouvée par des charges virales de contrôle persistantes indétectables et Ag HBe négatif. Cette observation illustre bien l'importance d'un traitement antiviral prophylactique au cours de l'immuno suppression. 


\section{PA17- Hépatites virales et anesthésie réanimation} I Miadi ${ }^{1}$, M Mokrani ${ }^{2}$

${ }^{1}$ Service d'anesthésie réanimation EPH Bouchegouf Guelma, ${ }^{2}$ Service de médecine du travail EPSP Bouchegouf Guelma

L'hépatite virale constitue un véritable problème de santé public ; le médecin anesthésiste-réanimateur est souvent amené à prendre en charge ces malades que ce soit en réanimation (en cas de complications) ou en anesthésie (chirurgie programmée ou dans la cadre de l'urgence). L'anesthésie d'un patient atteint d'une hépatite virale aigue ou chronique commence par une évaluation du degré de l'atteinte hépatique avec $t$ une recherche de complications et d'effets secondaires des traitements antiviraux. La prévention de la transmission nosocomiale des hépatites virale constitue la base de l'anesthésie réanimation de ces patients. La cirrhose, qui complique une hépatite a un retentissement multi viscérale qui demande une compréhension physiopathologique afin d'améliorer la prise en charge en anesthésie réanimation (agents anesthésiques et leurs modes d'administration). Cette revue de la littérature a pour objet de souligner la principales règles en anesthésie réanimation des patients atteints d'hépatites virales aux différents stades de cette maladie.

PA18- Profil d'auto-anticorps observés chez des patients avec suspicion d'hépatopathie auto-immune.

I Bedioune, Y Zemouli, A Guessoum, D Bougattoucha, S Hassanine, $N$ Touati, K Touamer, S Laref, AR Touabti.

Unité d'immunologie, Laboratoire Central de Biologie, CHU de Sétif.

Objectif: Les hépatopathies auto-immunes, représentées par la cirrhose biliaire primitive (CBP), les hépatites auto-immunes (HAI) et la cholangite sclérosante primitive (CSP) sont rares. Le diagnostic repose sur un faisceau d'arguments cliniques, biologiques, histologiques et immunologiques et sur l'exclusion d'autres étiologies. L'objectif de cette étude rétrospective était d'analyser le profil d'auto-anticorps observé chez des patients ayant une suspicion d'hépatopathie auto-immune. Patients, matériel et méthodes: Nous avons rétrospectivement analysé les données de 63 patients adultes ayant une forte suspicion d'hépatopathie auto-immune. L'Age moyen des patients était de 47,36 $\pm 20,64$ ans (16-84 ans)avec un sexratio $\mathrm{H} / \mathrm{F}=0,46$. Les patients avaient des anomalies hépatobiliaires cliniques et/ou biologiques d'origine indéterminée. Les principaux auto-anticorps fréquemment associés aux hépatopathies autoimmunes ont été recherchés sur des échantillons sériques. Les anticorps anti-nucléaires (ANA) ont été détectés par immunofluorescence indirecte (IFI) sur cellules HEp-2 (Biorad ${ }^{\circledR}$ ). Les anticorps anti-tissus non spécifiques d'organes: anti-LKM-1, antimitochondrie (AMA-2) et anti-muscles lisses (ASMA) ont été recherchés, par la même technique, sur coupes de tissus murins : foie, rein et estomac $\left(\right.$ Biorad $\left.^{\circledR}\right)$. Résultats : $33,33 \%(n=21)$ des sérums analysés étaient positifs pour les autoanticorps ciblés par la recherche (groupe 1). D'autres auto-anticorps (anti-réticuline ARA R1 et anti-cellules pariétales APCA) ont été découverts fortuitement dans 14,3\% des sérums (groupe 2). Le reste des sérums analysés $(52,38 \%)$ était négatif (groupe 3$)$. Nous avons noté une nette prédominance fémininedans le groupe 1 comparé au groupe 2 $(85,7 \%$ vs. $51,5 \% ; p=0.01)$. Les auto-anticorps associés aux hépatopathies auto-immunes se répartissent dans la population étudiée comme suit : 12,69\% AMA-2 seuls ;7,93\% ANA seuls ;7,93\% ASMA seuls $; 3,17 \%$ ANA+AMA-2 ; $1,58 \%$ ANA+ASMAet $0 \%$ anti-LKM1. Discussion: Les HAI et la CBP touchent préférentiellement les sujets de sexe féminin. La positivité des ANA et/ou des ASMA suggère une HAI de type 1. L'association des deux types d'anticorps rend le diagnostic encore plus probable surtout si une hypergammaglobulinémie est présente. Les anticorps anti-LKM1 sont associés à une HAI de type 2. Les AMA-2 sont très spécifiques de la CBP (plus de 95\% sont séropositives). En plus, certains ANA en sont également spécifiques. D'autres auto-anticorps, comme les p-ANCA et les antiSLA,devraient être recherchés en deuxième intention si les anticorps précédents sont négatifs. Conclusion: Les auto-anticorps ANA, ASMA, AMA-2 et anti-LKM1 sont un outil indispensable dansle diagnostic et la classification des hépatopathies auto-immunes. Cependant, leur absence lors d'un seul test n'exclut pas le diagnostic car leur titre peut varier au cours de l'évolution de la maladie. Par ailleurs, si ces auto-anticorps sont négatifs, d'autres auto-anticorps, moins fréquents, devraient être recherchés en deuxième intention.Enfin, il est important d'exclure une infection par le virus de l'hépatite $\mathrm{C}$ qui peut donner le même profil d'auto-anticorps.
PA19- Abcès du foie a germes pyogènes a propos de 09 cas. H Grini, S Bencharif, D Boudebza

Introduction: Ce sont des Collections purulentes, résultant d'un processus infectieux détruisant le parenchyme hépatique. Pathologie peu fréquente mais pouvant être très grave, mettant en jeu le pronostic vital par ses complications locales et systémiques. Les progrès des explorations morphologiques du foie ont contribué largement à l'amélioration du diagnostic, la thérapeutique et le pronostic des abcès hépatiques. Objectif: Etudier les aspects cliniques, morphologiques, microbiologiques et thérapeutiques des abcès pyogènes du foie. Matériels et méthodes : Etude série de cas. Service de maladies infectieuses a l'E.P.H Ibn Zohr, Guelma, de Janvier 2013 jusqu'au décembre 2014. Ont été inclus tous les patients présentant un abcès du foie à l'échographie et/ou à la TDM. Les données ont été colligées à partir des dossiers papiers des patients. Discussion : L'abcès du foie peut se voir à tout âge. La triade clinique de fontan n'est pas toujours présente. La recherche de la cause doit être systématique et précoce en fonction de l'état clinique du malade. Les anomalies biologiques sont fréquentes mais non spécifiques Le diagnostic d'abcès du foie repose essentiellement sur l'imagerie. Le traitement des abcès repose sur l' antibiothérapie qui est initialement probabiliste puis adaptée aux résultats microbiologique. Le traitement chirurgical est réservé aux échecs du traitement médical et à la cause de l'abcès si elle est chirurgicale. Conclusion : Les abcès $\mathrm{du}$ foie à germes pyogènes paraissent rares vu les difficultés diagnostiques. Le traitement reste multidisciplinaire comportant l'antibiothérapie, le drainage percutané ou la chirurgie à ciel ouvert dont les indications sont réduites

PA20- Le risque infectieux viral chez le polytransfusé : séroprévalence des virus de l'hépatite $B$ et $C$.

$S$ Chafai, N Djahmi, K Amoura, M Dekhil

Service de Microbiologie Clinique, CHU Dr Dorbon-Annaba

La transmission d'agents pathogènes par le sang ou ses dérivés est une préoccupation majeure en santé publique. Ce risque constitue un danger parfois mortel pour les patients transfusés, notamment de façon chronique. Il est principalement lié aux virus indétectables par sérologie au moment du don à cause de la fenêtre sérologique ou à cause de variants viraux. Dans le but de déterminer la prévalence des infections au VHB et au VHC chez les polytransfusés, nous avons réalisé une étude rétrospective au niveau du laboratoire de Microbiologie du CHU Annaba et portant sur les prélèvements de polytransfusés hospitalisés ou consultant au service d'Hématologie du CHU Annaba entre le 1 Janvier 2013 et le 31 Décembre 2014. Des tests immunoenzymatiques ont été utilisés pour le dépistage. La prévalence des anticorps anti-VHC chez ces patients était de 6,15\% avec un sexe ratio de 0,4. La prévalence de l'AgHBs était de 4,20\% avec un sexe ratio de 1,5. En conclusion, le risque résiduel de transmission des hépatites virales et du HIV après la transfusion sanguine persiste et nécessite des efforts supplémentaires en matière de sécurité transfusionnelle : vaccination contre l'hépatite $B$, amélioration des tests de dépistage chez les donneurs de sang, etc.

\section{PA21- Le risque transfusionnel infectieux lié à l'hépatite B} A Farouzi, A Tabet, O Seba

EPH Kouba laboratoire central CTS, Alger

En Algérie, la nécessité de gérer le risque transfusionnel infectieux lié à l'hépatite $\mathrm{B}$, étant donné que le seul marquer de dépistage obligatoire soit l'Ag HBs depuis le 24 Mai 1984. et dans un souci de sécurité transfusionnelle en vue de l'absence de détection du génome viral (DGV) dans une zone de moyenne endémie pour hépatite virale B (HBV) notre étude est axée sur deux objectifs : le premier est l'estimation du risque de transmission du VHB chez les donneurs de sang (DDS), le deuxième est l'évaluation de la prévalence de l'hépatite occulte chez les DDS ; pour ce, nous avons procédé par une étude prospective des échantillons vis-à-vis des marqueurs directs (AgHBs, AgHBe et l'ADN viral) et indirects (Anti-HBe l'Anti-HBs et anti-HBc) de l'hépatite B chez 2978 DDS total Par méthodes: Elisa (BOIRAD) pour les marqueurs sérologiques et au niveau du CTS de Kouba et MEIA (AXSYM) ainsi que par cobas* : AmpliPrep/COBAS* Taq Man* HBV test « ROCHE » au niveau de l'institut Pasteur d'Algérie, Ag HBs/Anti HBc+ est de 166 / 2978 soit 5,5\% (Pays à moyenne endémie VHB 2 à 7\%)20 DDS avait un anti HBC isolé soit 12\% par méthode 
ELISA, seules 04 échantillons ont été confirmés par MEIA au niveau de l'IPA soit $0,13 \%$ avait le profil anti HBc isolé, 1DDS avait une PCR positive; la prévalence de l'Hépatite $B$ occulte est de $1 / 2978$ soit $0,033 \%$ la prévalence de l'Hépatite occulte sont revue à la hausse en incluant les DDS à profil anti-HBc+/anti HBs+.Malgré le nombre de faux positifs donné pas le margeur anti-HBc; on préconise son introduction dans le cadre de l'absence du DGV des Produits Sanguin Labile, et ce, afin de réduire le risque de la transmission sanguine du VHB et de renforcer la sécurité transfusionnelle.

PA22- Evaluation de l'effet protecteur de l'homogénat du gastéropode terrestre Helix aspersa contre l'hépato-toxicité induite par un immunosuppresseur chez le rat

R Mebirouk ${ }^{1,2}$, Y Boussouf $^{1,2}$, NI Abdessemed ${ }^{1,2}$, D Naimi ${ }^{2,3}$

${ }^{1}$ Laboratoire de génie microbiologique \& application, Equipe de biologie et physiologie cellulaire \& moléculaire. ${ }^{2}$ Université des frères Mentouri - Route de Ain El Bey - Constantine - Algérie. ${ }^{3}$ Ecole Nationale Supérieure de Biotechnologie, Constantine - Algérie.

L'Helix aspersa est une espèce d'escargot comestible, elle est distribuée dans le nord africain et le sud de l'Europe. Elle a été depuis l'antiquité recommandée en médecine et préparée sous différentes formes pharmaceutiques. Des équipes de recherche se sont intéressées à l'étude des effets biologiques en particulier l'effet antioxydant, anti-inflammatoire et anticancéreux, des extraits préparés à partir de ce mollusque. Le but de notre recherche est d'évaluer l'effet protecteur de l'homogénat préparé à partir du pied de ce gastéropode (la chair) contre la toxicité induite par un immunosuppresseur de référence (la cyclosporine). Dans notre étude, nous avons travaillé sur des rats soumis à un traitement d'immunosuppresseur pendant 22 jours (le lot $\mathrm{C}$ ). Chez le lot $\mathrm{CH}$, on a administré la cyclosporine accompagnée par l'homogénat. Le lot $\mathrm{H}$ a reçu l'homogénat seul et enfin, lot témoin $\mathrm{T}$ a reçu de l'eau physiologique $(0,9 \%$.). Les poids des animaux ainsi que la quantité de l'aliment consommé ont été enregistré chaque jour et pendant la période des traitements. A la fin du traitement, un prélèvement sanguin é été effectué pour le dosage des transaminases. Les animaux sont ensuite sacrifiés pour prélever leurs foies pour la réalisation des coupes histologiques et le dosage du MDA. L'étude des coupes histologiques a montré que l'immunosuppresseur a induit une altération structurale dans le foie des animaux. Cette lyse cellulaire a diminué ou presque disparue dans le lot qui a reçu l'immunosuppresseur en association avec l'homogénat de l'escargot Helix aspersa. Quant aux résultats des examens biologiques (dosages enzymatiques, MDA), ils montrent que le lot de rats traités par immunosuppresseur et homogénat présente une diminution des transaminases, des Gamma-GT et du MDA. En conclusion, l'homogénat d'Helix aspersa pourrait contenir des molécules bioactives qui ont agit sur les cellules. Ces molécules pourraient apporter une protection des hépatocytes et pourrait être recommandée aux sujets soumis à un traitement immunosuppresseur.

PA23- Les méthodes non invasives d'évaluation de la fibrose hépatique au cours des hépatites virales $B$ et $C$

$S$ Boufarou, ML Meghadecha, A Mammeri, M Aidaoui, M Laouar

Service d'infectiologie, CHU Annaba

La fibrose hépatique est la principale conséquence lésionnelle au cours des hépatites virales chroniques $\mathrm{B}$ et $\mathrm{C}$ en termes de gravité et de pronostic. Le stade de fibrose est important à préciser dès le diagnostic d'une hépatite chronique et au cours de l'évolution du fait de son impact sur l'incidence des complications (la cirrhose et le carcinome hépatocellulaire) et sur le suivi médical. L'évaluation de la fibrose hépatique est d'une importance cruciale pour estimer le pronostic des hépatites virales chroniques $\mathrm{B}$ et $\mathrm{C}$, en termes de morbidité et de mortalité et décider ou non de la mise en route d'un traitement. Si l'examen de référence est la biopsie hépatique, de nouvelles méthodes non invasives d'évaluation de la fibrose ont pris une importance croissante dans la prise en charge des patients incluant les tests sériques et l'imagerie qui prend une place non négligeable surtout avec l'échographie dont le rôle dans l'évaluation de la cirrhose sur des critères morphologiques est bien connu; mais également par la mesure de la «dureté» du foie par l'élastographie impulsionnelle. But: citer et décrire les méthodes non invasives d'évaluation de la fibrose hépatique au cours des hépatites virales B et $\mathrm{C}$, son principe et son déroulement. Conclusion : Les méthodes non invasives d'évaluation de la fibrose hépatique sont simples, rapides, non invasives et performantes dans le diagnostic et l'évaluation de la maladie hépatique évitant ainsi le recours à la ponction biopsie hépatique sans toutefois la remplacer.

PA24- Epidémiologie de l'hépatite $C$ au niveau de L'EPH Ibn Zohr à Guelma , Algérie

Bouzenzen

Infectiologie, EPH Ibn Zohr, Guelma

Introduction : L'hépatite virale $\mathrm{C}$, appelée avant 1989 hépatite non A non $B$, constitue dans plusieurs régions du monde un problème de santé publique; et représente le type même de l'épidémie " silencieuse " que le dépistage et les thérapeutiques antivirales tentent de contrôler, surtout en l'absence de vaccination jusqu'à nos jours. La connaissance des caractéristiques épidémiologiques et de l'histoire naturelle de l'infection virale C est d'un grand apport pour la prévention de cette hépatite qui constitue une cause fréquente de cirrhose et de carcinome hépatocellulaire. Matériels et méthodes: Nous avons procédé a une étude chez 17 malades atteints de l'hépatite $\mathrm{C}$ au niveau du service des maladies infectieuses EPH Ibn Zohr à Guelma consécutifs, 08 hommes et 09 femmes, âge moyen 49ans : 1) la prévalence de l'infection par VHC en fonction de l'âge 2)la prévalence de l'infection par VHC en fonction du sexe 3)- la distribution des génotypes $d u$ virus. Résultats : Notions générale : Le virus de l'hépatite $\mathrm{C}(\mathrm{VHC})$ est un virus à ARN de la famille des flavivirus. Il comporte une enveloppe et une nucléocapside. Son génome comporte des gènes qui codent pour des protéines de structure (capside et enveloppe E1et E2), et d'autres pour des protéines non structurales (NS2, NS3, NS4, et NS5). Ce virus est caractérisé par un polymorphisme génétique important. Six génotypes numérotés de 1 à 6 ont été décrits avec plusieurs soustypes au sein de chaque génotype désignés par des lettres alphabétiques a, b, c, . . Au cours de l'hépatite C, la connaissance des génotypes a non seulement un intérêt épidémiologique mais aussi un intérêt pronostique et thérapeutique. La transmission du virus se fait essentiellement par voie le sang et ses dérivés. La transmission sexuelle est possible mais peu fréquente et la transmission verticale mère-enfant est exceptionnelle Jusqu'à 1996, la transfusion par du sang ou par ses dérivés représentait le mode de transmission essentiel du virus. Actuellement, la toxicomanie intraveineuse, certaines pratiques sociales à risques (scarifications, tatouages, piercing traditionnel...) et la contamination nosocomiale (soins dentaires, chirurgie, utilisation de seringue à usage multiple, endoscopie...) constituent les principaux modes de transmission virale. Après contamination par le VHC, 50 à $80 \%$ des patients restent porteurs du virus et développent une hépatite chronique $\mathrm{C}$ (HCC). L'évolution se fait progressivement vers la fibrose, la cirrhose et le carcinome hépatocellulaire. Prévalence mondiale de l'infection par le VHC : Il est estimé que 130 à 210 millions de personnes, soit $3 \%$ de la population mondiale, présentent une infection chronique par le VHC. La prévalence globale de l'infection par le VHC est cependant très variable sur le plan géographique. Elle est relativement élevée dans certains pays d'Afrique, de Méditerranée orientale, du Sud-Est asiatique et du Pacifique occidental, par rapport aux pays de l'Amérique du Nord et de l'Europe. A cette variation de prévalence s'associe une répartition variable des différents génotypes du V.H.C selon la zone géographique Le génotype $1 \mathrm{~b}$ est fortement prévalent au Japon (70 - $80 \%$ ) et en Europe (50 - $70 \%$ ) alors qu'aux états unis, il existe un mélange entre les génotypes $1 \mathrm{~b}$ et 1a. Le génotype 4 est de loin le plus fréquent en Afrique et au Moyen Orient, et particulièrement en Egypte. Les génotypes 2 et 3 s'observent surtout chez les toxicomanes. Enfin, le génotype 6 a été jusqu'à présent identifié à Hong-Kong. Prévalence de l'infection par le VHC selon le sexe à Guelma: on constate une prédominance de l'infection virale $C$ chez les sujets de sexe féminin $n=9(52,94 \%)$. Ceci pourrait être expliqué, au moins en partie, par certaines pratiques sociales plus communes parmi les femmes et connues par leur risque de transmission du VHC; c'est le cas du tatouage. Prévalence de l'infection par l e VHC selon l'âge a Guelma : il semble que l'infection par le VHC augmente avec l'âge et notamment chez les sujets de la cinquantaine. Cette donnée souligne le caractère horizontal essentiel de la transmission du VHC à Guelma. Génotypes et étude phylogénétique a Guelma: chez 17 patients infectés par le VHC; a été effectué un génotypage des isolats de VHC; une prédominance du génotype $1 \mathrm{~b} n=7 \quad(41,17 \%)$ suivi par les 
génotypes $2 \mathrm{n}=5(29,41 \%)$,puis génotypes 1a $n=3(17,64 \%)$ génotype 4 abc $n=1(5,88 \%)$ et génotype $5 a n=1(5,88 \%)$ (voir tableau 1$)$

Tableau 1 : prévalences de l'infection VHC en fonction du sexe et des génotypes.

\begin{tabular}{llllllll} 
& femme & homme & Géno 1a & Géno 1b & Géno 2 & Géno 4 & Géno 5 \\
\hline $\mathrm{n}=17$ & 9 & 8 & 03 & 07 & 05 & 01 & 01 \\
prévalencє & $52,94 \%$ & $47,06 \%$ & $17,64 \%$ & $41,17 \%$ & $29,41 \%$ & $05,88 \%$ & $05,88 \%$ \\
\hline
\end{tabular}

Conclusion : L'infection par le VHC semble augmenter avec l'âge, ce qui souligne le caractère essentiellement horizontal de la transmission virale, avec une preuve de contamination nosocomiale. Il existe une prédominance féminine, probablement en rapport avec certaines habitudes sociales plus communes chez les femmes, comme le tatouage. Les études phylogénétiques montrent une large prédominance du génotype $1 \mathrm{~b}$ dans la cohorte.

PA25- Un mode de révélation rare du kyste hydatique hépatique: la rupture intra péritonéale, à propos d'un cas

N Ghimouz, L Benlatreche, $N$ Boudada

EPH Zighoud Youcef Constantine.

L'hydatidose hépatique est fréquente dans nos contrées. La rupture dans la cavité péritonéale est un accident rare et grave réalisant des tableaux cliniques polymorphes. L'échographie et le scanner abdominal permettent le plus souvent un diagnostic facile et précis de cette complication. Nous rapportons le cas d'une patiente âgée de 52 ans prise en charge au service de chirurgie E P H Zighoud Youcef chez qui la rupture intrapéritonéale a été le mode de révélation du kyste hydatique hépatique.

PA26- prévalence de la co-infection VIH / VHC.E.H

C Labdouni, C Abderrahim, $N$ Mouffok Allab

Service des Maladies Infectieuses, CHU Oran

Objectifs : déterminer la prévalence de la co-infection VIH/VHC, les différents génotypes impliqués, les caractéristiques clinicobiologiques. Méthodologie: étude rétrospective des dossiers de patients co-infectés VHC/VIH durant la période de 2003 à 2014, en précisant les éléments épidémio-cliniques, biologiques, virologiques, thérapeutiques . Résultats : Trente et-une co-infections VIH/VHC sur 2392 PVVIH ont été colligées. Il s'agit de 20 hommes et 11 femmes. L'âge moyen est de 47,5 ans. Les génotypes les plus rencontrés étaient le génotype 1 (8/12), le VHC 1 a dans 5 cas et VHC 1 b, 2a2c dans 3 cas. et le génotype 4 dans un cas. La PCR était positive chez 26 patients (4.96 à $7.15 \mathrm{log}$ ). La co-infection avec le VHB a été notée dans deux cas. Comme co-morbidité, deux tuberculoses sous traitement, un diabète et Hypertension artérielle, un tabagisme chronique et éthylisme dans 3 cas et. Le fibro-test a conclu en une fibrose F0-F1/A0 chez 9 cas, F1-F2/A1 dans 6 cas, et sévère F4/A3 dans 6 cas. Le traitement a été instauré chez 16 patients. par bithérapie PEG-IFN alfa et Ribavirine, avec 8 réponses favorables, 3 cas d 'échec , 4 cas perdus de vue, 03 rechutes. Les effets secondaires ;toxicité hépatique en dans 2 cas, un cas d'hyperthyroidie, 2 cas d'anémie sévère. Discussion : Les génotypes les plus fréquents sont le 1 et le 2 . L'hépatite $\mathrm{C}$ chez les PVVIH est souvent active, l'échec thérapeutique sous ARV est constaté et la toxicité hépatique est réelle. Conclusion :La co-infection par les virus des hépatites et le VIH est un problème de santé publique évident, l'évolution a été favorable sans complication avec réponse complète dans la moitié des cas.

\section{PA27- Hépatites et traitement anti-tuberculeux}

$S$ benyahia

SCTMR Oued Zenati, Guelma

Le foie est un filtre entre le tube digestif et la circulation générale. Les médicaments absorbés par voie orale vont directement au foie ("premier passage") où ils sont "traités" par oxydation, réduction ou hydrolyse. Ce "traitement" est sous le contrôle de nombreux facteurs : rythmes biologiques, hormones, cytokines, maladies, facteurs génétiques, sexe, race, âge, état nutritionnel, autres médicaments ou produits chimiques. La découverte d'une anomalie hépatique lors du diagnostic d'une tuberculose peut évoquer une atteinte hépatique préexistante ou une localisation hépatique de la tuberculose (miliaire). Les effets secondaires hépatiques des médicaments représentent 7\% des effets secondaires des médicaments ; ils sont plus souvent en cause que l'hépatite virale dans l'insuffisance hépatique. Lors d'un traitement anti tuberculeux, l’atteinte hépatique peut revêtir trois aspects; ?? ?Hépatite an ictérique (nausées, vomissements, gène abdominale, fatigue) qui ne se manifeste que par l'élévation des transaminases, apparait dans les premières semaines de traitement et disparait spontanément. ?? ? ? Hépatite ictérique, ? ? ? Hépatite fulminante.

\section{PA28- Hépatite médicamenteuse toxique}

S Mekki

Département de pharmacie, faculté de médecine Annaba, Algérie

L'hépatite toxique médicamenteuse est une atteinte inflammatoire du foie, qui provoque la destruction de ces cellules, causée par la prise de façon délibérée ou non du médicament. Le foie est sensible à l'atteinte parce qu'il joue un rôle fondamental dans le métabolisme des médicaments qui pénètrent par le courant sanguin et subissent une transformation qui s'accompagne parfois de la formation de produits dérivés instables hautement toxique qui peuvent attaquer et léser le foie. Parmi les médicaments incriminés, citons le paracétamol, utilisé comme antalgique antipyrétiqu est indiqué dans le traitement de la fièvre et des douleurs, c'est le médicament le plus prescrit en Algérie. Il a l'avantage d'avoir peu d'effets indésirables lorsqu'il est prescrit a la posologie recommandée, mais en cas de surdosage, il devient toxique pour le foie provoquant ainsi des lésions qui peuvent devenir irréversibles voir mortelles. L'objectif est de faire une exploration des hépatites toxiques médicamenteuses, donc on a fait une description chimique de la molécule ainsi que de son mécanisme d'action et de son impact sur les fonctions hépatiques.

PA29- Intérêt des examens biologiques dans le diagnostic de l'hépatite auto-immune.

F Sekkache

Laboratoire de biochimie médicale, hôpital IBN- ZOHR Guelma.

Introduction : L'hépatite auto-immune (HAI) se définit comme une maladie inflammatoire chronique du foie de cause inconnue, pouvant survenir à tout âge. En l'absence de traitement, cette inflammation peut être responsable de nécrose, de fibrose et de cirrhose. L'hépatite auto-immune est associée à des anomalies de l'immunité sous forme d'auto-anticorps. Objectif : L'hépatite auto-immune est une maladie complexe et délicate à diagnostiquer, qu'on trouve souvent en conjonction avec une autre maladie biliaire, parfois seule. Le but de ce travail est d'évaluer l'apport des paramètres biologiques dans le diagnostic de l'hépatite auto-immune. Résultats et discussion: Chez un patient ayant une élévation chronique modérée des transaminases, le diagnostic de l'HAI repose sur les éléments suivants: il n'existe aucune cause habituelle de cette élévation de transaminases ; il existe une élévation des gammaglobulines et en particulier des IgG; il existe des auto-anticorps antinucléaire, les anticorps anti-muscle lisse, les anticorps anti-LKM, les anticorps antimitochondries et aussi les anticorps anti-SLA. Conclusion : L'Hépatite auto-immune est un diagnostic d'exclusion mais qui doit être évoqué à chaque fois devant une élévation des transaminases car méconnaitre ce diagnostic peut être fatal pour le patient, alors qu'un diagnostic précoce, compte tenu de l'efficacité des corticostéroïdes et des immunosuppresseurs entraine la guérison.

\section{PA30- Les Hépatites Chroniques Virales C et la nutrition F Sekkache}

laboratoire de biochimie médical, Hôpital IBN- ZOHR Guelma

Introduction : Le régime alimentaire est une composante importante de la gestion globale de L'Hépatite Chronique Virale C, il peut renforcer la réponse aux traitements et diminuer les effets indésirables de ces derniers. Objectif : L'objectif de ce travail est de considérer les malades infectés par le virus de l'hépatite $\mathrm{C}$ de la nutrition et du mode de vie le plutôt possible pour favoriser le bien être et la qualité de vie. Résultats et discussion: L'évolution de l'hépatite chronique $\mathrm{C}$ se fait vers la fibrose progressive, la cirrhose et le carcinome hépatocellulaire. Chez la majorité des patients porteurs d'une hépatite chronique $\mathrm{C}$, la progression vers la fibrose est lente et insidieuse. Les facteurs associés à une progression fibrosante rapide 
sont la consommation excessive d'alcool, un âge élevé, des coïnfections virales, un état de déficience immunitaire et un surpoids. Conclusion: Il est important pour les personnes infectées par l'hépatite chronique virale $\mathrm{C}$ d'obtenir un état nutritionnel optimal en équilibrant l'apport alimentaire, l'activité physique et les médicaments en vue de renforcer le système immunitaire et de favoriser la régénération du foie.

PA31- Profils sérologiques chez les patients en hémodialyse chronique au CHU Annaba

S Chafai, K Amoura, M Dekhil

Service de Microbiologie Clinique, CHU Dr Dorbon-Annaba

L'hémodialyse consiste en la diffusion du plasma dans le dialysât (mélange d'eau et d'électrolytes) au travers d'une membrane semiperméable. L'hémodialysé chronique a un risque augmenté de contamination par les virus de l'hépatite B (VHB) et C (VHC) et par le virus de l'immunodéficience humaine (VIH). La transmission se fait principalement de façon croisée entre patients. En plus, l'immunodépression associée à l'insuffisance rénale chronique favorise le passage à la chronicité des hépatites virales $\mathrm{B}$ et $\mathrm{C}$. Dans le but de déterminer la prévalence des infections virales au VHC, VHB et le VIH dans une population d'hémodialysés, une étude transversale a été réalisée en mars 2014 au sein de l'unité d'Hémodialyse du CHU Annaba. L'étude a porté sur 80 patients hémodialysés chroniques. Les sérums ont été testés par technique immuno-enzymatique (ELISA) au sein du laboratoire de Microbiologie du CHU Annaba. Malgré un échantillon modeste, le dépistage a permis d'obtenir une prévalence de $31,5 \%$ pour les anti-VHC et de $12,5 \%$ pour l'Ag HBs dans cette population. Elle est nulle pour le HIV. Tous les tests positifs ont été confirmés par un deuxième prélèvement testé par la même technique. En conclusion, la transmission nosocomiale est certaine dans les services d'hémodialyse et reste incontrôlée. Le dépistage régulier des patients séropositifs et le contrôle strict du respect des mesures d'hygiène doivent être institués dans les centres d'hémodialyse.

\section{PA32- Abcès hépatiques : À propos de 03 cas}

D Tliba, M Aidaoui, N Messalhi, ML Meghadecha, A Mammeri, M Laouar Service d'infectiologie, CHU Annaba.

Contexte : L'abcès hépatique est une collection purulente résultant d'un processus infectieux détruisant ou refoulant le parenchyme hépatique. Pathologie peu fréquente, pouvant être grave, mettant en jeu le pronostic vital par ses complications locales et systémiques. L'origine est surtout biliaire et l'utilisation de moyens endoscopiques est un facteur prédisposant pour la survenue des abcès hépatiques. Les progrès des explorations morphologiques du foie ont contribué à l'amélioration du diagnostic, permettant une prise en charge précoce. Le pronostic dépend de la rapidité diagnostique et thérapeutique. But: Etudier les aspects cliniques, morphologiques, et thérapeutiques des abcès hépatiques. Matériels et méthodes : Etude rétrospective basée sur l'analyse de 03 dossiers de patients présentant des abcès hépatiques hospitalisés au service d'infectiologie - CHU Annaba - sur une durée de 05 ans qui s'étend de l'année 2010 à l'année 2014. Résultats : L'âge moyen des patients était de 44 ans. Tous nos patients étaient de sexe masculin. Un de nos patients avait comme antécédent un diabète de type 2 . Les signes cliniques sont principalement la douleur de l'hypochondre droit, l'hépatomégalie, l'altération de l'état général et la fièvre. Les signes biologiques ne sont pas spécifiques. On note un syndrome inflammatoire chez tous les patients, un syndrome de cytolyse hépatique chez deux de nos patients, et un syndrome de cholestase hépatique chez un patient. Le foie droit était le plus atteint. Les aspects échographiques et scannographiques étaient polymorphes, essentiellement hétérogènes. Dans tous les cas, la prise en charge reposait sur une antibiothérapie. L'évolution était favorable pour un patient, les deux autres ont nécessité un recours à la chirurgie. Conclusion: Les abcès du foie paraissent peu fréquents. Le développement des moyens iconographiques et de la radiologie interventionnelle ont permis d'améliorer leur prise en charge.Les aspects radiologiques des abcès hépatiques sont polymorphes et dépendent du stade évolutif. Ils ne diffèrent pas de ceux rapportés dans la littérature. Leur prise en charge est multidisciplinaire comportant l'antibiothérapie, le drainage percutané ou la chirurgie à ciel ouvert dont les indications sont réduites.
PA33- Hépatites Virales : B et C chez les hémodialysés Chroniques à L'EPH d'Oued-Zénati

K Khacha

Service d'hémodialyse, EPH Oued-Zénati, Guelma

Introduction:Les hépatites virales demeurent les principales infections virales chez les hémodialysés. Leur gravité réside dans leur risque élevé d'évolution vers la chronicité et du développement d'une cirrhose ou d'un hépatocarcinome. Objectif : L'objectif de cette étude est de préciser la prévalence des hépatites virales $B$ et $C$ chez les hémodialysés chroniques au niveau du service d'hémodialyse de l'EPH d'oued-Zénati et d'en déterminer les principaux facteurs de contamination afin de contribuer à l'élaboration des moyens préventifs chez cette population à risque. Patients et méthodes : Nous avons mené une étude rétrospective auprès de 46 patients hémodialysés chroniques, au niveau de l'EPH d'Oued-Zénati depuis janvier 2005 jusqu'au décembre 2014. Nous avons inclus dans l'étude; l'ensemble des patients hémodialysés chroniques depuis plus de trois mois et sans limite d'âge. Nous avons exclu les patients ayant été dialysé de façon temporaire. Pour chaque patient ont été recueillis les données suivantes: âge sexe, néphropathie causale, sérologie antigène HBs et anticorps anti-HCV, âge de la dialyse et le nombre de culots globulaires transfusés. Résultats :L'âge moyen de nos patients était de 50 ans (25-82), avec un sex ratio de 1,3 en faveur du sexe masculin. La néphropathie causale était indéterminée chez $52 \%$ des patients et d'origine vasculaire chez $15 \%$ des patients. Sur les 46 patients, 14 étaient porteurs des virus VHC et ou VHB, soit (30\%) dont 12 infectés par le VHC soit (26\%), 4 infectés par le VHB soit $(8,69 \%)$ dont 3 guéris et 2 ayant une co-infection (VHC-VHB) soit $(4,34 \%)$. Chez les patients infectés par le VHC et ou le VHB, la durée moyenne de l'hémodialyse était de 8 ans contre 3 ans chez les patients non infectés. Le nombre moyen de culots globulaires reçu chez les patients infectés était de 8 contre 2 chez les patients non infectés. Conclusion: L'infection par le VHC demeure l'infection virale la plus prépondérante chez l'hémodialysé chronique, l'ancienneté de l'hémodialyse ainsi que les transfusions sanguines répétées constituent les principaux facteurs associés à la contamination. Pour réduire l'incidence de ces infections, une meilleure observance règles d'hygiène ainsi qu'une stratégie d'isolement des patients infectés s'avèrent nécessaires. De plus, il faut limiter le recours aux transfusions sanguines au profit de l'utilisation de l'érythropoiétine recombinante.

PA34- Stratégies thérapeutiques dans la prise en charge et la prévention du carcinome hépatocellulaire

$W$ Abdaoui, A Gouri, DE Benouareth

Département de science de la vie et de la nature, Faculté des sciences de la vie et de la nature Guelma.

Le diagnostic du carcinome hépatocellulaire est souvent tardif à cause de l'apparition des maladies hépatiques sous-jacentes (cirrhose ou hépatite). Les choix thérapeutiques restent restreints pour les patients avec un stade avancé. Il est important de souligner que le carcinome hépatocellulaire est une tumeur qui nécessite une prise en soin multidisciplinaire (interniste, radiologiste, chirurgien, pathologiste, oncologue, pharmacien). Alors la contribution de chacun de ces cliniciens peut améliorer la prise en charge et la qualité des soins des patients. Objectifs : déterminer le rôle du clinicien / pharmacien dans : les modalités du dépistage précoce du carcinome hépatocellulaire, la connaissance de différentes classifications proposées pour stadifier le $\mathrm{CHC}$, la connaissance des avantages et limites de l'algorithme thérapeutique BCLC. Contrairement aux autres tumeurs solides où le pronostic se base sur la classification TNM, il n'y a pas de classification consensuelle pour le CHC. Plusieurs modèles de stadification ont été proposées (Okuda, BCLC, CLIP, TNMAJCC...) mais les études les comparant ont donné des résultats discordants. Pour l'inclusion dans les essais thérapeutiques en situation palliative, il a été ainsi montré que la classification du CLIP. Bien que la classification TNM décrive la propagation du cancer du foie, les décisions relatives au traitement se basent plus souvent sur la résécabilité de la tumeur. Seule la classification BCLC présente un algorithme thérapeutique qui facilite les décisions et choix de traitement

PA35-Épidémiologie de l'hépatite B au Maghreb H Brahimi, M Bensaad, S Benchouk, A Benabdellah 


\section{Hôpital universitaire, Maladies infectieuses, Ain Temouchent.}

Introduction: L'hépatite $\mathrm{b}$ représente un véritable problème de santé publique dans les pays du Maghreb, Algérie, Libye, Mauritanie, Maroc et Tunisie. Elle est le type même d'épidémie silencieuse que le dépistage et les thérapeutiques antivirales tentent de contrôler. L'objectif est décrire les aspects épidémiologiques de l'hépatite b au Maghreb. Matériels et méthodes : Nous avons procédé à une revue de la littérature en utilisant la base de données Pub Med recherchant les articles originaux et les mises au point en langue anglaise et française. Résultats : Au Maghreb, La prévalence du portage de l'Ag HBs varie entre 1,8 et 4,9\% avec un nombre estimatif de 2,7 millions de personnes atteintes, le génotype $D$ est prédominant ainsi les mutations dans la région pécore du génome VHB sont très répandues. Les données rapportées sont hétérogènes, reflétant la diversité de l'environnement, des facteurs socio-économiques et culturels, en plus, l'épidémiologie du virus VHB n'est pas connue avec précision. L'Algérie se trouve dans une région de prévalence intermédiaire selon l'OMS, la prévalence du portage de l'Ag HBs est de $2,18 \%$ en 1998 et 1,53\% en 2006 et ceci grâce à un dépistage systématique dans le cadre d'un don de sang, un bilan préopératoire, un examen prénuptial ou d'un contrôle prénatal. Cependant, Le Maroc, pays de faible endémicité, la prévalence de l'Ag HBs est de 1,66\% dans une population active marocaine suite à une étude de dépistage massif durant la période 2006-2009, et dont les facteurs de risque sont dominés par les comportements sexuels à risque (43,84\%). En Libye, la prévalence globale est de $2,2 \%$ suite à une enquête séro épidémiologique nationale 2004-2005, la scarification et certaines pratiques traditionnelles sont incriminées dans leur survenue. En Tunisie, elle est de 2,9\%, la présence d'un membre de la famille infectés par le VHB, les pratiques de scarification, les pratiques de seringues dans le centre de soins de santé primaires et le sexe (masculin) ont augmenté de façon significative le risque du portage chronique de l'infection. Néanmoins, en Mauritanie, zone à forte endémicité, la prévalence de l'hépatite B est de $25 \%$, la toxicomanie, l'excision, les infections sexuellement transmissibles et l'acupuncture semblent également jouer un rôle important dans la fréquence élevée de l'infection à VHB. Au total, Cette situation épidémiologique nécessite des interventions actives évidemment répandues en matière de prévention et de contrôle, le programme de vaccination anti-hépatite $B$, seul moyen efficace, devrait être appliqué avec la plus grande discipline dans les cinq pays.

PA36- Intérêt du fibroscan dans le dépistage de la fibrose hépatique chez les patients atteints d'hépatite virale chronique O.F Zaoui, N Mouffok, MH Labdouni

Service d'infectiologie CHU ORAN

Introduction : Le fibroscan permet l'étude non invasive de la fibrose hépatique et a été proposé dans le suivi des malades atteints d'hépatopathie chronique. Le but de cette étude était d'évaluer les résultats obtenus par l'utilisation de cet outil par le service d'infectiologie du CHUO chez des malades atteints d'hépatite chronique virale. Patients et Méthodes: Il s'agit d'une étude transversale et descriptive, réalisée au sein du service d'infectiologie du CHUO sur une période allant du mois de Décembre 2014 au mois de Mars 2015, incluant des patients atteints d'hépatites virales chroniques (hépatite $\mathrm{B}, \mathrm{C}$ et co-infectés $\mathrm{HIV} / \mathrm{HCV}$ ) et suivis à notre niveau. Au cours de cette étude, nous avons utilisé un fibroscan echosens 502, deux sondes (medium, xl). Résultats : Durant cette période, 71 patients ont été inclus, dont l'âge moyen était de 44 ans, avec une prédominance féminine. Parmi ces patients, 51 étaient suivi pour HCV, 17 pour une HBV et d'autres sont des patients présentant une co-infection HCV/HIV. Les tests étaient réussis chez $97 \%$ des patients qui ont subi l'examen. Interquartile range/médiane (IQR/M) $<$ à 0,3 chez 56 patients. Concernant la stadification de la fibrose : hépatite virale $B$ : 5 patients étaient classés $F 4,3 F 3$; hépatite virale $\mathrm{C}: 15$ patients étaient classés $\mathrm{F} 4,3 \mathrm{~F} 3 \mathrm{~F} 4,3 \mathrm{~F} 3$; hépatite virale $\mathrm{C} / \mathrm{HIV}$ : 3 patients étaient classés F0F1. Conclusion: Compte tenu de sa facilité d'emploi et de sa très bonne acceptabilité par les malades ; l'élastométrie pourrait constituer un outil très intéressant pour le suivi de la fibrose hépatique non seulement chez les malades non traités que chez ceux traités. Il est probable que le fibroscan devienne dans un futur proche un outil incontournable dans l'évaluation non invasive de la fibrose en pratique clinique.
PA37- Particularité du foie chez le sujet âgé

$Z$ Khelifi, H Gouasmia, S Maizi, Y Boucherit

Laboratoire de Physiologie, Faculté de Médecine, Annaba

Le vieillissement est un processus physiologique lent et progressif. Il découle de facteurs intrinsèques et environnementaux. La structure et les fonctions de l'organisme varient à partir de l'âge mûr. Ces modifications débutent par un déclin des fonctions organiques avant l'apparition des années après des signes extérieurs du vieillissement des tissus et de l'aspect général du corps. Plusieurs mécanismes, en particulier le stress oxydatif et le raccourcissement télomérique provoquent le vieillissement hépatique qui se caractérise par une diminution du poids, de la taille et du volume du foie. Avec épaississement de la capsule et tendance du foie à mouler les organes de voisinage. L'accumulation de grains de lipofuscine lui confère une coloration brune. La réduction du flux sanguin hépatique est expliquée par les changements de l'endothélium des sinusoïdes hépatiques (pseudo-capillarisation). Microscopiquement, on note une réduction du nombre des hépatocytes et des mitochondries alors que leurs tailles augmentent. Accroissement du volume des lysosomes, avec accumulation du produit terminal de la peroxydation lipidique (lipofuscine). Sans omettre l'amoindrissement du réticulum endoplasmique lisse et de l'activité enzymatique microsomale. Fonctionnellement, du fait de la réduction de la masse musculaire et du taux d'hémoglobine la bilirubinémie diminue. L'activité des phosphatases alcalines s'accroît, probablement en raison de l'ostéoporose. La synthèse des selles biliaires baisse alors que celle du cholestérol reste normale, voire augmentée. L'albumine diminue indépendamment du statut nutritionnel. Cependant en dehors de l'albuminémie il n'y a pas de modification significative des tests biologiques. De ce fait toute anomalie de ces tests chez le sujet âgé est pathologique. L'ensemble de ces variations influence le métabolisme et l'excrétion hépatique des médicaments. D'où la nécessité de réduire leur posologie de 30 à $40 \%$ chez les personnes de plus de 75 ans.

PA38- Séroprévalence de l'hépatite virale Delta dans l'extrême Est algérien

ML Meghadecha ${ }^{1}$, S Boulanouar ${ }^{3}$, R Mabrouk ${ }^{4}$, F Drardja $^{2}$, A Zerdazi $^{1}$, M Laouar $^{1}$

${ }^{1}$ Université Badji Mokhtar ,CHU, Annaba; ${ }^{2} \mathrm{EPH}$ Bouchegouf; ${ }^{3} \mathrm{EPH}$ Ibn Zohr, Guelma ; ${ }^{4}$ EPH Azzaba, Skida, Algérie

Contexte : L'hépatite B chronique est un problème de santé publique en Algérie avec une séroprévalence de 2,15\% (enquête Nationale de l'Institut Pasteur d'Alger, 1998) mais les études sur la séroprévalence du VHD sont quasiment absentes. But : Déterminer la séroprévalence du VHD dans l'extrême Est algérien. Matériels et méthodes : C'est une étude transversale de séroprévalence sur 201 patients suivis pour hépatite virale B.Les critères d'inclusions sont les patients suivis pour AgHBs positifs soit dans le cadre d'une hépatite $\mathrm{B}$ aigue ou chronique durant la période du 01/01/2013 au 31/09/2014. Etude multicentrique effectuée dans quatre services d'infectiologie. La recherche du VHD a été faite par sérologie(ELISA) avec titrage des Anticorps totaux anti VHD. Résultats: Age moyen des patients était de 43,55 ans $\pm 14,90$ ans, $56 \%$ de sexe masculin, $97 \%$ étaient algériens, deux de la Guinée équatoriale, deux du Ghana, un du Mali et un du Niger. La résidence des patients était répartie sur six wilayates de l'extrême Est algériens. La séroprévalance du VHD était de 1,49\% (IC à 95\%: 0,31\% 4,30\%) soit trois patients, parmi eux deux étaientoriginaires de la Guinée équatoriale et présentaient une coïnfection par le virus Delta avec des anticorps de type IgM positif et une charge virale Delta élevée et le troisième patient était algérien et ses anticorps de type IgM ainsi que la charge virale Delta étaient négatifs. Conclusion: La séroprévalence de l'hépatite Delta trouvé dans notre étude (1,49\%) est faible, ce travail mérite d'être complété dans le cadre d'études multicentriques à caractère national pour déterminer la séroprévalence de cette hépatite Delta en Algérie.

PA39- Prévalence des hépatites B et $C$ chez les donneurs de sang au CTS de Sétif

S Bounaas ${ }^{1}$ I Bedioune ${ }^{1}$, Bouzidi ${ }^{2}$, R Arif $^{2}$

${ }^{1}$ Laboratoire Central de Biologie, CHU Saadna Abdenour, Sétif; ${ }^{2}$ Centre de Transfusion Sanguine, CHU Saadna Abdenour, Sétif 
Introduction : Les hépatites virales $B$ et $C$ restent un défi mondial (400 millions de porteurs de l'Ag HBs et 170 millions de séropositifs pour le VHC dans le monde). Leurs prévalences varient d'un pays à l'autre. L'Algérie appartient à la zone de moyenne endémicité avec une prévalence de $2,16 \%$ pour l'Ag Hbs et de $1 \%$ pour VHC dans la population générale. Notre étude consiste à évaluer la prévalence de l'infection par les virus de l'hépatite B (AgHbs) et l'hépatite C (AC anti VHC) chez les donneurs de sang au CTS de Sétif durant l'année 2014. Patients et Méthodes : Il s'agit d'une étude rétrospective portant sur 10980 dons Homologues récoltés pendant l'année 2014 au CTS de Sétif. Les caractéristiques relatives à l'âge, le sexe et le type de collectes ont été relevées afin de déterminer les facteurs influençant le statut viral des donneurs de sang. La détection des marqueurs viraux a été effectuée par des techniques immunoenzymatiques de type ELISA ou MEIA. Chaque résultat positif a été réévalué par une deuxième détermination puis confirmé au niveau de l'Institut Pasteur d'Algérie. Résultat : 38 dons positifs ont été dépistés pour les deux marqueurs, soit une prévalence totale de $0,34 \%$.Ces cas positifs sont répartis en 11 cas VHC positifs et 27 cas HBs positif avec une prévalence respective de $0,1 \%$ et de $0,24 \%$. L'âge moyen des donneurs séropositifs pour les virus des hépatites $B$ et $C$ était de $34,28 \pm 8,86$ ans avec des extrêmes de 19 à 50 ans. Nous avons noté une nette prédominance masculine (82\%) avec un sexe ratio de $7 / 31$ soit $0,2.57 \%$ des cas positifs ont été recrutés en collecte mobile $(22$ donneurs) contre 43\%(16 donneurs) sélectionnés au CTS. Nous n'avons noté aucune co-infection VHB-VHC. Discussion : dans notre étude les prévalences de l'hépatite $B(0,24 \%)$ et $C(0,1 \%)$ chez les donneurs de sang sont nettement inférieur à ceux publié dans une étude de l'ANS en 2008 qui estimait la prévalence des hépatites b et c en Algérie chez les donneurs de sang de 1,09\% et 0,49\% respectivement. L'âge moyen des cas positifs est toujours retrouvé chez l'adulte jeune dans la tranche d'âge de 20- 49 ans, active sexuellement. Les donneurs séropositifs pour les virus des hépatites $\mathrm{B}$ et $\mathrm{C}$ sont sélectionnés aussi bien au niveau du camion mobile qu'au niveau du CTS. Conclusion: La séroprévalence des marqueurs infectieux des hépatites B et C chez les donneurs de sang au CTS de Sétif pour l'année 2014 reste faible mais discutable tant que ne sont pas rendu obligatoire le dosage des ALAT et la recherche des Ac anti Hbc. Par ailleurs, il est nécessaire de modifier la stratégie de récolte du sang pour mieux rentabiliser le don et diminuer le risque résiduel et promouvoir ainsi la sécurité transfusionnelle.

PA40- Effet du bouclier glycanique de l'enveloppe du VHC sur l'infectivite et la neutralisation autologue lors de l'infection chronique

M Arab ${ }^{1}$, C Gaudy-graffin 1,2, A Goudeau ${ }^{1,2}$.

${ }^{1}$ INSERM U966, Faculté de Médecine. Tours, France. ${ }^{2}$ Service de Virologie, Hôpital Bretonneau, CHRU de Tours. France.

Après la contamination par le VHC, 70\% des cas évoluent vers la chronicité. Les anticorps neutralisants sont des acteurs clés dans la défense de l'hôte. La variabilité des glycoprotéines d'enveloppe et leur importante glycosylation est en partie à l'origine de l'échappement à la réponse immunitaire. L'étude de la réponse neutralisante autologue analysant la sensibilité des variants circulant chez les porteurs chroniques est pertinente dans ce contexte. Dans ce travail, sept sérums provenant de patients porteurs chroniques du VHC ont été sélectionnés. Les gènes d'enveloppe $\mathrm{E} 1 / \mathrm{E} 2$ ont été amplifiés par RT-PCR nichée. La présence des sites de glycosylation a été vérifiée. Les enveloppes amplifiées ont servi pour la production de pseudo-particules rétrovirales. Des tests d'infectivité et de neutralisation avec le sérum autologue ont été réalisés. Sept enveloppes possédant tous les sites de $\mathrm{N}$-glycosylation ont été amplifiées. Seule une particule entrait dans les cellules cible. Les essais de neutralisation montrent que cette dernière avait une sensibilité réduite à la neutralisation autologue.

PA41- L'Hépatite C chez les Hémodialysés : Enjeux Thérapeutiques et Perspectives

MEH Labdouni, OF Zaoui, S Benzoubara, C Abderrahim, A Kaid, $N$ Mouffok

Services des maladies infectieuses CHU Oran

Introduction et objectifs : La prévalence du VHC est élevée chez les patients hémodialysés chroniques, engendrant ainsi un problème important de prise en charge. L'objectif de ce travail est de décrire les caractéristiques épidémio-cliniques de ce type de patients ainsi que de la difficulté de leurs prise en charge. Matériels et méthodes : Étude rétrospective et prospective de dossiers des patients HCV positifs pris en charge au sein de service des Maladies Infectieuses. Résultats : vingt-deux patients hémodialysés ayant une PCR-HCV positive , 11 étaient de sexe féminin et 10 de sexe masculin, la classe modale de l'âge était celle de [35;45] ans : 8 patients $(28,56 \%)$. Les génotypes rencontrés étaient essentiellement le $1: 20$ patients, le sous type $1 \mathrm{~b}$ était retrouvé dans $2 / 3$ des cas, 2 patients étaient du génotypes $2(7,14 \%)$. La charge virale était comprise entre 5-7 log chez 13 patients $(46,41 \%)$.et supérieur à $7,5 \log$ chez 5 patients $(17,85 \%)$. Un cas de co-infection HBV. Le traitement était initié chez 18 patients $(64,26 \%), 4$ patients étaient exclu de l'étude 1 décès 1 perdu de vue, et chez 2 patients le traitement est non encore entamé). La bithérapie (INF+RBV) était instaurée chez 16 patients (88,8\%), la monothérapie (INF) chez 2 patients, l'interféron alpha 2 b était utilisé chez 12 patients $(66 \%)$ ). les incidents au TRT étaient dominés par le syndrome pseudo-grippal, les arthralgies chroniques tout au long du TRT pour 2 patients, syndrome du canal carpien, hypertrophie cardiaque chez 1 patient. L'anémie était fréquente chez 14 patients, elle était importante chez 8 cas ; ayant nécessité l'arrêt définitif du TRT chez 5 cas patients et temporaire chez 3 . Six patients avaient reçu des transfusions et 07 de l'EPO. La dose de RBV était diminuée à $200 \mathrm{mg} 3$ fois / semaine. La thrombopénie et la leucopénie était légère dans la quasi-totalité des cas. Trois patients étaient non répondeurs(16\%), 03 avaient rechuté $(16 \%)$ et 07 patients avaient achevé une RVS $(38,5 \%)$. Conclusion : la prise en charge des hémodialysés HCV + requière une gestion optimale des effets secondaires essentiellement d'ordre hématologique (anémie) laissant suggérer l'utilisation d'une dose réduite de RBV ainsi qu'une bonne préparation des patients avant l'initiation du TRT.

\section{ABSTRACTS DES POSTERS LIBRES}

PA42- Experimental study on the effect of Cupressus sempervirens planted in the East of Algeria in an animal model I Bouguenoun ${ }^{1}, Z$ Bensakhri ${ }^{1}$, SE Merzoug ${ }^{1}, W$ Bouguenoun ${ }^{2}, D$ Bendjeddou ${ }^{1}$ and $M$ Houhamdi ${ }^{1}$

${ }^{1}$ Laboratoiry of biology, water and environment (LBEE), University 8 May 1945 Guelma- Algeria ; ${ }^{2}$ Laboratory of biochemistry and applied microbiology, University of Badji Mokhtar Annaba- Algeria

Cupressus sempervirens is one of the most widespread species in Algeria which have a very high allergenic capacity. We studied how the different fraction of pollinic extract of this species can modulate the immune response of mice. Following sensitizing, the mice leukocyte's formula revealed a decrease in the rate of the neutrophils and an increase in the eosinophils. As for broncoalveolar lavage, a significant increase in the cell population was recorded. The histological study showed major modifications in the structure of the lungs. We can say that these results demonstrate an inflammatory allergy.

PA43- Evaluation de l'activité antibactérienne des huiles essentielles de Laurus nobilis, sur des souches de Pseudomonas aeruginasa résistantes aux antibiotiques.

M Bouras , N Boutafnouchet, $S$ Khadri

Laboratoire de Biochimie et de Microbiologie appliquée, faculté des sciences, Université Badji Mokhtar, Annaba 23000, Algérie

P.aeruginosa est une bactérie pathogène opportuniste, clairement reconnue comme un pathogène nosocomial. Cette espèce a également une remarquable capacité de résister aux antibiotiques. Le fait le plus inquiétant de ces dernières années est le signalement de plus en plus fréquent de souches de P.aeruginosa résistantes à un nombre croisant d'antibiotiques et même dans certains cas des souches totalement résistantes à l'ensemble des molécules disponibles; ces bactéries constituent, ainsi, un problème majeur de santé publique Afin de trouver un remède pour le problème de la multirésistance aux antibiotiques, les plantes médicinales ont été employées en phytothérapie, vue leur richesse en centaines, voire en milliers de composants de valeurs thérapeutiques; parmi les quels les huiles 
essentielles contenant les plus importants principes actifs des plantes aromatiques. Dans notre travail, nous nous sommes intéressés à l'isolement et l'identification des souches appartenant à l'espèce P.aeruginosa à partir des prélèvements de pus et des urines. Nous avons étudié le comportement de nos souches vis-à-vis des antibiotiques testés pour P.aeruginosa afin d'évaluer leur résistance, également nous avons étudié l'activité antibactérienne de l'huile essentielle de Laurier d'Apollon (Laurus nobilis) de l'est algérien. L'activité antibactérienne a été testée sur 20 souches d'origine pathologique et une souche de référence de P.aeruginosa par la méthode de diffusion en disque, cette méthode a révélé que l'huile essentielle n'a pas montré une zone d'inhibition ni pour les 20 souches testées ni pour la souche de référence, inversement il y est montré une forte activité vis-à-vis de 5 souches de Staphylococcus aureus testées. Par ailleurs, ce travail ne constitue qu'une ébauche et peut faire l'objet d'autres recherches plus approfondies s'appuyant sur un nombre élevé de prélèvements ainsi sur un ensemble de plusieurs extraits aromatiques.

\section{PA44-Activites antimicrobiennes de l'huile essentielle d'origanum vulgare 1 \\ SE Djouad, A Neghra}

Laboratoire de chimie thérapeutique, département de pharmacie, faculté de médecine, université Badji Mokhtar Annaba

L'Origanum vulgare L est une plante de la famille des lamiacées ou labiées, elle possède d'innombrables propriétés thérapeutiques dont l'activité antimicrobienne fait l'objet de notre étude. Des résultats promettant ont été obtenus par l'étude qualitative de l'activité antifongique et antibactérienne de l'huile essentielle de notre Origanum vulgare $\mathrm{L}$ ayant inhibé toutes les souches fongiques testées et certaines souches bactériennes réputées pathogènes et relativement résistantes aux antibiotiques. L'exploitation des résultats préliminaires font appel aux essais complémentaire de détermination de la concentration minimale inhibitrice et fongicide afin d'envisager leur utilisation aussi bien en milieu hospitalier qu'en communautaire.

PA45- Evaluation des paramètres du stress oxydant chez les diabétiques insulinodépendants

S Boukertouta

Le diabète est une maladie systémique fréquente affectant une tranche importante de la population mondiale. Il a été suggéré que la production accrue de radicaux libres et le stress oxydatif est un événement majeur pour le développement des complications du diabète. La forte réactivité des ERO détermine les changements chimiques dans pratiquement tous les composants cellulaires, conduisant à la peroxydation des lipides et la modification des protéines et a une éventuelle perturbation des défenses antioxydantes chez les sujets diabétiques. Notre travail vise à mettre en évidence la variation des paramètres métaboliques, le statut antioxydant et oxydant chez des diabétiques insulinodépendants (DID). Dans la population diabétique étudiée par rapport aux témoins, le profile lipidique était inchangé, de même, les paramètres sériques exceptés les taux de la glycémie et de l'hémoglobine glyquée où une augmentation significative a été noté chez les DID. Aucune différence significative de la concentration sérique du statut oxydant et antioxydant n'a été observée chez les patients DID. Les résultats suggèrent qu'une courte durée d'évolution du diabète n'est pas directement impliquée dans la surproduction des radicaux libres.

PA46- Evaluation des maladies à déclaration obligatoires (MDO) au niveau de l' EPH IBN ZOHR durant l'année 2014

S Boulanouar, ML Megadecha, AG HaridI

Université Badji Mokhtar,CHU Annaba, Annaba

Introduction: Les maladies à déclaration obligatoire (MDO) présentent une préoccupation majeure pour les autorités sanitaires. La déclaration est un moyen de surveillance adéquat à fin de protéger la santé des populations et de disposer d'un réseau d'information sur un problème sanitaire publique jugé important. Objectifs : avoir une vision sur le profil épidémiologique de la région, évaluer le dispositif actuel mis pour la lutte contre les MDO. Matériels et méthodes :
Type d'étude: étude descriptive rétrospective. Matériels d'étude : fichiers des déclarations des MDO. Durée d'étude : 12 mois (janvier 2014- décembre 2014). Résultat : L’EPH Ibn Zohr a recensé durant l'année 2014 : 210 MDO, Hépatites B : 122 cas soit 58,095 pour cent ; Hépatite A : 36 cas soit 17,142 pour cent avec un pic en octobre 2014: 14 cas; Hépatite C: 33 cas soit 15,714 pour cent; Méningites : 10 cas soit 4,761 pour cent; Brucellose : 09 cas soit 4,285 pour cent. Un pic des MDO en mois de janvier, Une recrudescence en mois de décembre. Les régions les plus touchées en mois de mars 2014: Guelma: 43 pour cent, Fedjoudj: 22 pour cent, Oued znati, oued cheham, bouati, lakhzara, djeballah : 07 pour cent. Conclusion : la déclaration reste un moyen primordial pour la lutte contre les MDO, le dispositif de lutte contre les hépatites B , A reste à renforcer par une sensibilisation et la motivation de tous les moyens pour arriver à obtenir l'objectif essentiel c'est l'éradication complète des hépatites.

PA47- Tolérance a l'imatinib, dasatinib et nilotinib dans le traitement de leucémie myéloïde chronique. W Soudani ${ }^{1}$, R Djafer ${ }^{2}$, S Boughrira ${ }^{3}$, H Djeddi ${ }^{3}$, F Griffi $^{3}$

1 Laboratoire de Chimie Thérapeutique, Département de Pharmacie,Faculté de Médecine Annaba. ${ }^{2}$ Service de Toxicologie,CHU Annaba. ${ }^{3}$ Service d' Hemato - oncologie CHU Annaba.

Introduction : Les Inhibiteurs de Récepteurs à Tyrosine Kinase sont des nouvelles cibles thérapeutiques récemment explorées dans le but d'augmenter la sélectivité d'action anti-tumorale et de réduire ainsi les effets indésirables et toxiques du traitement anticancéreux. L'Objectif principal était d'évaluer la tolérance aux Inhibiteurs de Récepteurs à Tyrosine Kinase chez les cancéreux. Matériel et Méthode: Une étude descriptive rétrospective a été réalisée sous forme d'une enquête sur une série de trente trois cas (33) de patients cancéreux atteints de Leucémie myéloïde chronique suivis au niveau du service d'hémato- oncologie CHU Annaba avec un âge moyen de 45 ans et deux extrêmes de 17 à 72 ans, dans la période allant du mois de Décembre 2013 au mois d'Avril 2014. Résultats et Discussion: La fréquence des effets communs liés à la chimiothérapie antérieure a été très élevée $(91,30 \%)$ pour les patients ayant reçu l'Imatinib : Imatib ${ }^{\circledR}$, ceci a été expliqué par le fait que la fréquence des patients ayant reçu une chimiothérapie en première intention avant l'inhibiteur de Tyrosine Kinase est plus élevée pour ce groupe $(54,54 \%)$; ce qui permet de constater l'influence de chimiothérapie antérieure sur les effets indésirables des inhibiteurs de Tyrosine Kinase. L'étude de la tolérance a montré que l'Imatinib : Glivec ${ }^{\circledR}$ a donné un seul cas avec des complications type colopathie et allergie respiratoire; avec disparition des douleurs osseuses et l'hypertension artérielle obtenue avec l'Imatinib: Imatib $\AA$; ce qui confirme une bonne tolérance de la spécialité de Glivec $\AA$ par rapport à l'Imatib $₫$. Nous n'avons noté aucune complication (effet indésirable cumulatif) pour Dasatinib et Nilotinib, avec diminution des effets spécifiques (le Dasatinib a donné $16,67 \%$ des douleurs osseuses, le Nilotinib 9,09\% de douleurs osseuses et 9,09\% de myalgies), diminution de l'asthénie ( le Dasatinib a induit 33,33\% et le Nilotinib 9,09\% ) ; ceci témoigne d'une meilleure tolérance des nouvelles molécules inhibitrices de récepteurs à Tyrosie Kinase Dasatinib et Nilotinib par rapport à la molécule de référence Imatinib. Conclusion : A travers ces données nous relevons l'intérêt de sélection des protocoles thérapeutiques et l'importance d'une surveillance stricte des effets indésirables et toxiques des thérapeutiques ciblées. Nous insistons aussi sur l'intérêt d'une étroite collaboration entre clinicien et pharmacien, afin d'assurer un meilleur suivi thérapeutique pour les cancéreux.

\section{PA48- La dysthyroidie}

ML Hamlaoui, H Mermouli

La dysthyroïdie est le mauvais fonctionnement de la glande thyroïde qui se traduit par un trouble de la sécrétion des hormones thyroïdiennes influant sur le mécanisme basale de l'organisme en perturbant plusieurs fonctions telles que cardiaque et hépatique; plus ou en moins, variable selon le type et l'intensité de l'atteinte. Cette pathologie est fréquente s'accroisse avec l'âge et touche des millions de personnes dans le monde dont la particularité sont les femmes. Certaines pathologies de la thyroïde peuvent être traitées avec des médicaments, tandis que, pour d'autres, la chirurgie est le 
meilleur traitement. Au-delà des effets secondaires, la maladie a des conséquences socio-professionnelles comme une sorte de double peine pour la personne malade et son entourage.

\section{PA49- Fibroblastome desmoplastique de l'espace parapharynge àpropos d'une observation}

A Farhi, A Daoudi, S Zitouni, N Djerad, S Kharoubi, S Douche, A Saidia

Service ORL et chirurgie de la face et du cou, CHU Annaba

Le fibroblastome desmoplastique (FD) est une tumeur des tissus mous, bénigne, rare et de croissance lente. Elle a une distribution anatomique large et touche essentiellement l'adulte de sexe masculin. Elle se caractérise macroscopiquement par un aspect typique pseudocartilagineux et histologiquement par une prolifération de fibroblastes réguliers souvent de forme stellaire dans un stroma collagénique ou myxo-collagénique dense. Les auteurs rapportent un nouveau cas siégeant au niveau de l'espace parapharyngé, qui illustre les aspects anatomocliniques particuliers de cette entité rare. Ils essayent de cerner à travers une observation originale de FIBROBLASTOME DESMOPLASTIQUE à localisation parapharyngée les problèmes diagnostiques et thérapeutiques de cette tumeur au niveau de cette topographie.

PA50-Amylose systémique révélée par un syndrome d'hypertension portale (à propos d'un cas).

$N$ Ghimouz, L Benlatreche, $N$ Boudada

EPH Zighoud Youcef Constantine.

Il s'agit d'un patient âgé de 34 ans, admis pour investigation d'une ascite de grande abondance avec altération de l'état général. Après examen clinique et une série d'examens biologiques, et radiologiques le diagnostic d'un syndrome d'hypertension portale est posé. Puis dans le cadre de la recherche étiologique, le malade à bénéficier d'une laparotomie exploratrice avec biopsie des adénopathies profondes et du foie; et l'examen anatomopath a objective une amylose hépatique. Devant ce tableau, on a complété le bilan d'extension à la recherche d'autres localisations possibles. Donc, cette maladie de surcharge assez rare, surtout dans sa forme primitive mérite d'être étudiée ; d'ou cette mise au point.

\section{PA51- Sélénium, les deux facettes d'un oligo-élément essentiel} Z Benyahia

Le sélénium est considéré comme un élément à double face : à dose faible il est un nutriment essentiel à la vie des animaux et des humains, par contre à doses élevées, il devient toxique. Toutes les cellules de l'organisme en contiennent dans des concentrations qui varient d'un tissu a l'autre et qui sont en rapport avec la teneur de la ration. Des apports insuffisants dans les aliments aboutissent à des carences très fréquentes dans les zones séléniprives du globe qui entraînent chez l'homme le développement des maladies cardiovasculaires et le cancer. Cependant, un apport exagéré en Se dans les aliments, souvent rencontré dans les régions sélénifères, entraîne des sélénoses toxiques. La plupart des auteurs ont attribué au Se des rôles antioxydant, anti radicaux libres et immunostimulant. Il joue aussi un rôle dans le métabolisme au niveau du foie et contribue au maintien des muscles squelettiques et cardiaques et des spermatozoïdes, mais les mécanismes des effets préventifs du sélénium sur le cancer ne sont pas encore bien établis.

PA52- Complications infectieuses après chirurgie majeure des cancers pharyngo-laryngés

A Farhi, A Daoudi, N Djerad, S Zitouni, S Kharoubi, S Douche, A Saidia Service Orl et Chirurgie de la face et du cou C H U Annaba Algérie

Le développement de la médecine moderne est associé à l'apparition de nombreuses pathologies iatrogènes liées à la difficulté d'améliorer simultanément la qualité de toutes les procédures de soins et d'entretien. Notre activité opératoire est en partie basée sur la chirurgie carcinologique cervicale des cancers pharyngo-laryngé. Ces interventions comportent une période post-opératoire très lourde, l'infection hospitalière reste le paramètre le plus grave de cette période. Cette étude est rétrospective portant sur 472 chirurgie totales, ou partielle du carrefour pharyngo-laryngée sur une période de 17ans (1996-2014).

PA53- Les Chironomidae (Diptera: insecta): un allergène majeur causant l'asthme allergique

A Zeynat Bensakhri ${ }^{1}$, K Zerguine ${ }^{1,2}$, I Bouguenouna ${ }^{3}$, D Bendjeddoua ${ }^{3}$

${ }^{1}$ Laboratoire de biologie, eau et environnement, université 8-Mai1945, Guelma, Algérie; 2 Département d'écologie et génie de l'environnement, université 8-Mai-1945, Guelma, Algérie; 3 Département de biologie, université 8-Mai-1945, Guelma, Algérie

L'asthme allergique est une maladie inflammatoire des bronches et des voies aériennes, liées à l'inhalation des allergènes inspirés « les pneumallergènes ». Dans notre travail, nous nous sommes intéressé d'étudier les effets allergiques d'une famille des insectes appelée les Chironomidae. Ces insectes, pendant le stade larvaire de son cycle biologique sont pourvus d'hémoglobine, responsable des manifestations d'hypersensibilité immédiate, principalement l'asthme. Pour la réalisation de ce travail, on a préparé l'extrait brut de ces larves pour déterminer leur effet allergique sur le système immunitaire d'un modèle murin. Pour cela, les souris traitées ont reçu des instillations intranasales de l'extrait larvaire afin d'étudier les différentes paramètres immunologiques influencés (la cytologie du sang périphériqueet lacomposition cellulaire des liquides de lavage nasal et broncho-alvéolaire) ainsi que l'histologie des poumons. Dans l'ensemble, cette expérimentation a permis de mettre en évidence le pouvoir inflammatoire de l'extrait larvaire sous forme d'inflammation localisée au niveau des voies respiratoires.

PA54- La propagation des bactéries multi-résistantes (BMR) dans les milieux hospitaliers de la wilaya de Guelma

W Bouguenoun ${ }^{1}$, AA Bentorki ${ }^{2}$, I Bouguenoun ${ }^{3}$, T Merad $^{1}$

${ }^{1}$ Laboratoire de microbiologie et biochimie appliquée - Université BADJI Mokhtar -Annaba- Algérie ; ${ }^{2}$ Laboratoire de bactériologie Hôpital Ibn Zohr- Guelma-Algérie ; ${ }^{3}$ Laboratoire de biologie, eau et environnement - Université 8 mai 1945 -Guelma- Algérie.

L'hôpital est un lieu où l'on traite mais c'est également un lieu où l'on peut contracter des maladies infectieuses que l'on appelle les infections nosocomiales, rançon du progrès en matière de techniques diagnostiques et thérapeutiques; par ailleurs, l'émergence de souches multi-résistantes posant des problèmes d'impasses thérapeutiques, a compliqué la prise en charge de bon nombre de ces infections acquises à l'hôpital. Dans notre approche, nous avons étudié pendant six mois la propagation des souches bactériennes résistantes aux antibiotiques au niveau des hôpitaux de la wilaya de Guelma, les bactéries ont été collectées auprès des patients hospitalisés depuis plus de 48 heures (urine, pus, hémoculture,...) et dans l'environnement de l'hôpital (les surfaces du bloc opératoire, surfaces des salles de séjours des malades, mains du personnel soignant, chariot de soin, sanitaire...). Ces isolats ont fait l'objectif d'une étude phénotypique effectuée par les techniques usuelles de Microbiologie, ceux ayant répondu aux critères caractéristique de chaque bactérie sont repiqués et identifiés par l'utilisation de la galerie biochimique API $20 \mathrm{NE}$, l'API $20 \mathrm{E}$ et Api Staph (Bio Mérieux, France), avec une étude complémentairede l'antibiogramme par la méthode de diffusion des disques. Durant la période allant du 01/ 2014 au 06/ 2014, un total de soixante-trois (63) souches multi résistantes ont été isolées: 16 Staphylococcus aureus résistant à la méticilline, 39 entérobactéries et 08 bacilles Gram négatif non fermentaire. Mots clés : infection, nosocomiale, bactérie, résistance, antibiotique.

PA55- Evaluation de l'Activité Antibactérienne et de l'Effet Génotoxique du Bis (benzyl) sulfamide et du Cyclo- Bis (benzyl) sulfamide Néosynthétisés

I Becheker1, H Berredjem1, M Berredjem2, A Ladjama1

${ }^{1}$ Laboratoire de Biochimie et Microbiologie appliquées, Université Badji Mokhtar - Annaba; ${ }^{2}$ Laboratoire de Chimie Bio-Organique Appliquée, Université Badji Mokhtar - Annaba

La synthèse de nouvelles molécules antibactériennes, en réponse à l'évolution croissante de la résistance bactérienne, est l'une des alternatives actuelles pour pallier aux insuffisances des antibiotiques disponibles. Cependant, la présence d'un risque lié à l'effet mutagène doit être déterminée par l'évaluation du potentiel génotoxique de ces molécules. Le test d'Ames est l'un des tests les plus simples, rapides et facilement adaptable pour l'évaluation de l'effet mutagène d'un 
produit et ainsi la sureté préclinique d'un médicament. Le but de cette étude est d'évaluer l'activité antibactérienne de deux molécules innovantes de sulfamide, le Bis (benzyl) sulfamide et le Cyclo-Bis (benzyl) sulfamide, sur plusieurs souches cliniques multirésistantes, ainsi que le potentiel génotoxique éventuel par le test d'Ames. L'activité antibactérienne est évaluée qualitativement par la méthode de diffusion sur milieu solide Mueller Hinton en mesurant les diamètres des zones d'inhibition, et quantitativement en déterminant la CMI sur milieu liquide. L'effet génotoxique est évalué in vitro par le test d'Ames en utilisant trois souches standards Salmonella typhimurium TA 98, TA 100 et TA 1535 avec et sans activation enzymatique utilisant la méthode de préincubation. Les résultats montrent que, Les deux molécules présentent une bonne activité antibactérienne vis-à-vis des souches cliniques utilisées. Avec les trois souches de S. typhimurium, le Bis(benzyl) sulfamide ainsi que le Cyclo- Bis(benzyl) sulfamide ne montrent aucun effet génotoxique. En conclusion, les deux nouvelles molécules peuvent offrir de réelles perspectives thérapeutiques. Néanmoins, des essais complémentaires de pharmacocinétique seront nécessaires pour mieux caractériser ces molécules.

PA56- Enquête ethnobotanique sur l'usage des plantes médicinales pour soigner l'ictère

NI Benkirat, N Boughandjioua, M Bouachari, F Mastour, A Benkirat, S Bouriachi, FZ Boualleg

Laboratoire de Pharmacognosie, Département de Pharmacie, Faculté de Médecine, Université Badji Mokhtar, Annaba

La phytothérapie s'appuie sur des traditions millénaires, elle constitue un des fondements de la médecine populaire en Afrique qui demeure un recours important pour certaines personnes. Elle a été transmise d'une génération à l'autre par la communication orale. L'enquête ethnobotanique s'avère indispensable pour la connaissance du traitement de certaines maladies par les plantes médicinales tel que l'ictère: coloration jaune de la peau, de la sclérotique et des muqueuses due à l'accumulation dans le sang, de bilirubine (libre ou conjuguée), dont les étiologies sont variables ; anémie hémolytique, syndrome de Gilbert, hépatite virale ou toxique, infection bactérienne, parasitose, cirrhose, tumeur maligne, lithiase infectée). Le but de notre étude est la détermination de l'usage traditionnel des plantes médicinales pour soigner l'ictère. Pour cela on a préparé un questionnaire de 10 questions distribué sur 100 personnes, d'âges différents, dans trois wilayas de l'est algériens (GUELMA, ANNABA, SKIKDA); réalisée entre Novembre 2014 et Février 2015. Les résultats ont permis d'identifier un nombre important de drogues dont les plus utilisées sont les écorces du Daphné garou (Lazaaz), l'alaterne, l'origan, du pois chiche, les dattes et le miel, l'artichaut sauvage (karnoun), en grand pourcentage, elles sont utilisées par application directe, macération et infusion. La durée du traitement est jusqu'à guérison (de 30 à 40 jours). Les enquêtés utilisant ses plantes, confirment leurs efficacité surtout pour le Daphné garou, l'alaterne l'origan et l'artichaut sauvage. Les perspectives de notre travail sont de mener une étude pharmacologique préliminaire, ainsi que déterminer le mécanisme d'action et le ou les principes actifs responsable de l'activité, ouvrant la voie à leur usage en nature ou sous forme galénique pour soigner l'ictère.

\section{PA57- Responsabilité médicale et infections nosocomiales}

R Noura

médecine légale, Etablissement public hospitalier Ibn Zohr, Annaba

De nombreuses infections liées à la pratique médicale, aux conséquences imprévisibles et parfois même dramatiques, sont de plus en plus signalées à nos services .Ces infections peuvent survenir dans n'importe quel lieu de soins (public ou privé). De tels accidents évitables de surcroît, sont inadmissibles et ne doivent plus jamais se reproduire. Tous les médecins, et en particulier les chefs de service médicaux et paramédicaux, ainsi que les gestionnaires de santé, sont tenus pour premiers responsables dans de telles situations. Le non respect et la non application et suivi des dispositions réglementaires prévues par le sinstructions $\mathrm{n}^{\circ}$ 64/MSP du 17/11/1998 portant création du comité de lutte contre les infections nosocomiales (CLIN), $\mathrm{n}^{\circ} 573 / \mathrm{MSP} / \mathrm{DP}$ du 13/12/2000 relative à l'hygiène au niveau des centres d'hémodialyse, et $n^{\circ} 398$ /MSP/CAB du 12/09/1995 relative à la gestion des déchets hospitaliers, ainsi que les recommandations du guide technique d'hygiène hospitalière de1998 sont source de responsabilité au sens des Articles 239 et 266 du code de la santé, l'article 6 du code de déontologie et les article 441 bis, 442 bis et 443 du code pénal.

PA58- Résistance aux antibiotiques des germes isolés des hémocultures au service d'hématologie CHU Annaba

L Nait Merzoug, D Dardar, K Amoura, S Amiri, A Otmane, N Djahmi, $M$ Dekhil

Service de Microbiologie CHU Dr. Dorban, Annaba

Introduction: Les services d'hématologie reçoivent un nombre important de patients infectés immunodéprimés. Chez ces derniers le pronostic des bactériémies est particulièrement sévère et souvent associé à un fort taux de mortalité. Une antibiothérapie probabiliste en accord avec l'écologie du service doit donc être rapidement mise en route. L'objectif : de ce travail est de déterminer la fréquence des bactéries isolées à partir des hémocultures reçues du service d'hématologie «CHU Annaba » et d'évaluer leur profil de résistance Matériel et méthodes : Il s'agit d'une étude rétrospective effectuée au niveau du laboratoire de microbiologie CHU Dorban Annaba. Cette étude porte sur les bactéries isolées des hémocultures provenant du service d'hématologie, du 1er janvier 2013 au 31 décembre 2014. L'étude de la sensibilité des germes aux antibiotiques a été réalisée selon les recommandations du CLSI. Les doublons ont été éliminés de l'étude (plusieurs flacons positifs au même germe chez un patient). La contamination a été définie par la présence d'une hémoculture positive unique à une bactérie commensale chez les patients non immunodéprimés. Résultats : 321 hémocultures ont été analysées, 75 se sont révélées positives. Le sexratio était de 1.67. L'étude bactériologique a montré une prédominance des Entérobactéries avec $42.7 \%$ des isolats, l'espèce la plus fréquente été Escherichia coli (34.4\%) suivi de Klebsiella pneumoniae $(31.25 \%)$. Les autres germes étaient principalement des Staphylocoques (28\%) dont $80 \%$ étaient des Staphylococcus coagulase négative (SCN),Pseudomonas aeruginosa (13.4\%), Acinetobacter baumannii (9.2\%) et Enterococcus sp (6.7\%) . 50\% des Entérobactéries étaient résistantes aux céphalosporines de 3éme génération par production de Beta lactamase à spectre étendu (BLSE), 88.2\% des SCN étaient résistants à l'oxacilline tandis que toutes les souches d'Acinetobacter baumannii étaient résistantes à l'imipenème. Conclusion : Les infections en hématologie impliquent de plus en plus de germes résistants. Leur pronostic dépend de la rapidité du traitement. Enfin leur prise en charge nécessite la connaissance de l'écologie locale afin d'optimiser l'antibiothérapie probabiliste.

PA59- Qualité physico-chimique et bactériologique des eaux de sources de la région de Guelma

B Hamlaoui ${ }^{1}$, M Behailil ${ }^{2}$, H Laraissia ${ }^{2}$

${ }^{1}$ Université Larbi Ben M'hidi Oum El Bouaghi ; ${ }^{2}$ Université 08 Mai 1954 Guelma

La région de Guelma est caractérisée par la présence de plusieurs sources naturelles ; ce qui donne à la population de la wilaya la possibilité de s'alimenter avec une eau meilleur, tandis qu'elle manifeste un mécontentement envers la qualité actuelle des eaux potables. C'est pourquoi on doit s'assurer de sa bonne qualité non seulement du coté bactériologique mais aussi de ses caractéristiques physico-chimiques. L'étude est portée sur l' analyse bactériologique et physico-chimique de quatre sources durant deux mois Avril et Mai de l'année 2011, au niveau des laboratoires de Microbiologie au sein du département de Biologie et de Chimie dans le département de Génie des Procédés; qui mènent principalement à l'identification des germes totaux, des bactéries indicatrices de la contamination fécale et des germes pathogènes ainsi que les concentrations des éléments physico-chimiques. Les résultats obtenus montrent que ces eaux de sources étudiées sont de bonne qualité physico-chimique, mais la qualité bactériologique est plus au moins acceptable ayant pour majeur cause la négligence des autorités en charge et la population.

PA60-Fréquence de vente des produits lights dans la région d'annaba. année 2013

M Megueddem, R Djafer, AB Messaoudene

Laboratoire de Toxicologie. Faculté de Médecine d'Annaba. 
Introduction/Objectif : Une chose est sûre, le Light prend une place de plus en plus considérable dans notre alimentation et l'utilisation de l'Aspartame s'est généralisée dans la plupart des produits que nous consommons. Nous nous sommes intéressés dans ce travail, réalisé dans la région d'Annaba à la disponibilité et la fréquence de vente de ces produits dans certains commerces de proximité. Matériels et méthodes : Il s'agit d'une étude descriptive portant sur une série de 100 commerçants choisis au hasard au sein des différents quartiers du chef-lieu de la wilaya d'Annaba, durant le premier trimestre de l'année 2013. L'étude est réalisée sur la base d'un questionnaire comportant 17 items. Résultats : Plus de la moitié des commerçants interrogés (soit 68,42\%) ont affirmé vendre les produits Lights dans un but de régime alimentaire, 95\% ont déclaré vendre ces produits, essentiellement sous forme de boissons gazeuses. 78.94\% des ventes concernaient les produits allégés en Aspartame et la fréquence de vente de ces produits, selon $61,34 \%$ des commerçants, est élevée. Conclusion: L'intérêt que portent ces commerçants pour ces produits prouve à quel point la demande est devenue importante au fil des années. Mais aujourd'hui, et à cause de la continuité du débat concernant l'innocuité ou la toxicité de
l'Aspartame, plusieurs organisations alimentaires et sanitaires préfèrent éviter ce point de conflit et s'attachent à promouvoir d'autres édulcorants naturels ou de synthèse dont l'efficacité et la sécurité ont été clairement établies.

PA61- Les lymphomes non hodgkiniens extra ganglionnaires a localisation ORL à propos de 40 cas

A Farhi, A Daoudi, N Djerad, S Zitouni, S Kharoubi, S Douche, A Saidia Service ORL et chirurgie de la face et du cou, Annaba

Le lymphome non hodgkinien( $\mathrm{LNH})$ extra ganglionnaire de la sphère ORL est les plus fréquents après les localisations digestives. Le LNH Type B (85\%) l'emporte largement sur le LNH type T (15\%). L'origine de cette pathologie serait une translocation chromosomique. Les facteurs environnementaux et les infections virales joueraient un rôle important dans son étiopathogénie. Le traitement qui repose essentiellement sur la chimiothérapie exige au préalable un diagnostic histologique et un bilan d'extension précis. L'objectif de cette étude est de préciser les particularités épidémiologiques, radio anatomiques et pronostiques.
Ce recueil des abstracts a été publié dans le "Batna Journal of Medical Sciences » BJMS, l'organe officiel de "l'association de la Recherche Pharmaceutique - Batna »

Le contenu de la Revue est ouvert « Open Access » et permet au lecteur de télécharger, d'utiliser le contenu dans un but personnel ou d'enseignement, sans demander l'autorisation de l'éditeur/auteur.

Pour plus d'informations, contacter BatnaJMS@gmail.com

ou connectez-vous sur le site de la revue : www.batnajms.com 